Instituto de Astronomia, Geofísica e Ciências Atmosféricas Universidade de São Paulo

\author{
Aline A. Vidotto
}

\title{
Three-Dimensional Numerical Simulations of Magnetized Winds of Low-Mass Stars
}

São Paulo, Brazil

October 2009 



\section{Aline de Almeida Vidotto}

\section{Three-Dimensional Numerical Simulations of Magnetized Winds of Low-Mass Stars}

A thesis presented in partial fulfilment of the requirements for the degree of Doctor in Science in Astrophysics at Universidade de São Paulo, Brazil.

Advisor: Dr. Vera Jatenco Silva Pereira

São Paulo, Brazil

October 2009 

To my sister and my brother 

not seen. que se não vêem.

Hebrews 11:1

Hebreus 11:1 


\section{Abstract}

he subject of this thesis is the mass loss of low-mass stars through magnetized coronal winds. Stellar winds have been a topic of extensive research in Astrophysics for a long time, and their first investigations focused on the solar wind. Nowadays, we know that the magnetic field plays a crucial role in the acceleration and heating of coronal winds. Despite of the knowledge of the fine structure of the solar magnetic field, much less information is known regarding the configuration of the magnetic field in other stars. In this thesis, we investigate the structure of the magnetic field in the coronae of solar-like stars and young stars by means of three-dimensional magnetohydrodynamical numerical simulations. We self-consistently take into consideration the interaction of the outflowing wind with the magnetic field and vice versa. Hence, from the interplay between magnetic forces and wind forces, we are able to determine the configuration of the magnetic field and the structure of the coronal winds.

We investigate solar-like stellar winds and their dependence on the plasma- $\beta$ parameter (the ratio between thermal and magnetic energy densities). This is the first study to perform such analysis solving the fully ideal three-dimensional magnetohydrodynamics equations. We adopt in our simulations a heating parameter described by $\gamma$, which is responsible for the thermal acceleration of the wind. We analyze winds with polar magnetic field intensities ranging from $B_{0}=1$ to $20 \mathrm{G}$ and we show that the wind structure presents characteristics that are similar to the solar coronal wind. The steady-state magnetic field topology for all cases is similar, presenting a configuration of helmet streamer-type, with zones of closed field lines and open field lines coexisting. Higher magnetic field intensities lead to faster and hotter winds. The increase of the field intensity generates a larger "dead zone" in the wind, i.e., the closed loops that inhibit matter to escape from latitudes lower than $\sim 45^{\circ}$ extend farther away from the star. The Lorentz force leads naturally to a latitude-dependent wind. We show that by increasing the density and maintaining 
$B_{0}=20 \mathrm{G}$, the system recovers to slower and cooler winds. For a fixed $\gamma$, we show that the key parameter in determining the wind velocity profile is the $\beta$-parameter at the coronal base. Therefore, there is a group of magnetized flows that would present the same terminal velocity despite of its thermal and magnetic energy densities, as long as the plasma- $\beta$ parameter is the same. This degeneracy, however, can be removed if we compare other physical parameters of the wind, such as the mass-loss rate. We also analyze the influence of $\gamma$ in our results and we show that it is also important in determining the wind structure.

We further investigate magnetized stellar winds of low-mass pre-main-sequence stars. In particular we analyze under which circumstances these stars present elongated magnetic features (e.g., helmet streamers, slingshot prominences, etc). We focus on weaklined T Tauri stars, as the presence of the tenuous accretion disk is not expected to have strong influence on the structure of the stellar wind neither on the coronal magnetic field. We show that the plasma- $\beta$ parameter is a decisive factor in defining the magnetic configuration of the stellar wind. Using initial parameters within the observed range for these stars, we show that the coronal magnetic field configuration can vary between a dipole-like configuration and a configuration with strong collimated polar lines and closed streamers at the equator (multicomponent configuration for the magnetic field). We show that elongated magnetic features will only be present if the plasma- $\beta$ parameter at the coronal base is $\beta_{0} \ll 1$.

Using our self-consistent three-dimensional magnetohydrodynamical model, we estimate for the stellar winds of pre-main-sequence stars the timescale of planet migration due to drag forces exerted by the stellar wind on a hot-Jupiter (i.e., on a giant planet that orbits very close to the star). Our model suggests that the stellar wind of these multicomponent coronae are not expected to have significant influence on the migration of hot-Jupiters. 


\title{
Resumo
}

\begin{abstract}
a
tópico abordado nesta tese é a perda de massa através de ventos coronais magnetizados em estrelas de baixa massa. Ventos estelares têm sido estudados extensivamente há vários anos, tendo inicialmente como foco o vento solar. Atualmente, sabe-se que o campo magnético é essencial na aceleração e aquecimento dos ventos coronais. Apesar do conhecimento detalhado que temos da estrutura magnética do Sol, pouco se sabe sobre a configuração do campo magnético em outras estrelas. Nesta tese, é investigada a estrutura do campo magnético nas coroas de estrelas do tipo solar na Seqüência Principal e de suas predecessoras na pré Seqüência Principal através de simulações numéricas magneto-hidrodinâmicas tri-dimensionais. Aqui, consideramos de forma auto-consistente a interação entre o vento e o campo magnético e vice-versa. Dessa forma, pela interação entre forças magnéticas e forças do vento, consegue-se determinar a configuração do campo magnético e a estrutura dos ventos coronais.
\end{abstract}

Realizamos um estudo de ventos de estrelas do tipo solar e a dependência dos mesmos com o parâmetro $\beta$ do plasma (a razão entre as densidades de energia térmica e magnética). Este é o primeiro estudo a realizar tal análise resolvendo as equações tri-dimensionais da magneto-hidrodinâmica ideal. Em nossas simulações, adotamos um parâmetro de aquecimento descrito por $\gamma$, que é responsável pela aceleração térmica do vento. Então, nós analisamos ventos com intensidades de campo magnético nos pólos no intervalo de $B_{0}=1$ a $20 \mathrm{G}$ e mostramos que a estrutura do vento apresenta características que são similares à do vento coronal do Sol. No estado estacionário, a topologia do campo magnético obtida é similar para todos os casos estudados, apresentando uma configuração do tipo helmet streamer, com zonas de linhas fechadas e abertas de campo magnético co-existindo. Intensidades mais altas de campo levam a ventos mais acelerados e mais quentes. O aumento na intensidade do campo gera também uma "zona morta" maior no vento, i.e., os loops fechados que previnem que a matéria escape da coroa em 
latitudes menores que $\sim 45^{\circ}$ se estendem a maiores distâncias da estrela. Além disso, mostramos também que a força de Lorentz gera naturalmente um vento que é dependente da latitude. Ao aumentar a densidade da coroa mantendo $B_{0}=20 \mathrm{G}$, mostramos que o sistema volta a apresentar ventos menos acelerados e mais frios. Para um valor fixo de $\gamma$, mostramos que o parâmetro essencial na determinação do perfil de velocidade do vento é o parâmetro $\beta$ calculado na base da coroa. Dessa forma, acredita-se que haja um grupo de ventos magnetizados que apresenta a mesma velocidade terminal independentemente das densidades de energia térmica ou magnética, desde que o parâmetro $\beta$ seja o mesmo. No entanto, essa degenerescência pode ser removida ao se comparar outros parâmetros físicos do vento, tal como a taxa de perda de massa. Nós também analisamos a influência do $\gamma$ nos nossos resultados e mostramos que ele é importante na determinação da estrutura do vento.

Além disso, investigamos ventos magnetizados de estrelas de baixa massa da pré Seqüência Principal. Em particular, analisamos sob quais circunstâncias tais estrelas apresentam estruturas magnéticas alongadas (e.g., helmet streamers, proeminências do tipo slingshot, etc). Focamos especialmente em estrelas do tipo T Tauri fracas, uma vez que o tênue disco de acreção, quando presente ao redor de tais estrelas, não deve causar forte influência na estrutura do vento estelar e nem na do campo magnético coronal. Nós mostramos que o parâmetro $\beta$ do plasma é um fator decisivo na configuração do campo magnético do vento estelar. Usando parâmetros iniciais adequados ao que se é observado para tais estrelas, nós mostramos que a configuração do campo magnético pode variar entre uma configuração semelhante a de um dipolo e uma configuração com linhas fortemente colimadas em torno do eixo polar e streamers fechados ao redor do equador (configuração de multi-componentes para o campo magnético). Mostramos que as estruturas alongadas do campo magnético somente estão presentes se o parâmetro $\beta$ do plasma na base da coroa é $\beta_{0} \ll 1$.

Usando nossos modelos magneto-hidrodinâmicos, auto-consistentes, tri-dimensionais, estimamos para ventos de estrelas da pré Seqüência Principal a escala temporal de migração planetária devido a forças de arraste exercidas pelo vento em um planeta tipo hot-Jupiter (i.e., um planeta gigante que orbita muito próximo da estrela). Nosso modelo sugere que os ventos estelares de coroas com multi-componentes de campo magnético não têm influências significativas na migração de hot-Jupiters. 


\section{Acknowledgement}

Fould like to express my sincere gratitude to those who, directly or not, gave me the possibility to complete this thesis.

I acknowledge FAPESP (04/13846-6) and CAPES (4686/06-3) for the financial support in the development of this work.

I am deeply indebted to my advisor Dr. Vera Jatenco-Pereira and my unofficial coadvisor Dr. Merav Opher. I thank you very much for the motivation, patience, stimulating suggestions, and insightful discussions during all the time of research. I appreciate the time you invested in my formation, and above all, I am grateful for your friendship. I furthermore thank Dr. Elisabete de Gouveia dal Pino for her suggestions and discussions during the follow up of this work, and Dr. Peter L. Biermann for his contagious enthusiastic view of Science, for the stimulating discussions, and advice during these years. I thank Dr. Tamas Gombosi for allowing the use of BATS-R-US, and Dr. Gerd Weigelt for the always welcoming receptions at the Max Planck Institute for Radioastronomy (Bonn). I thank the staff at the supercomputer Columbia (NASA/Ames) where the simulations presented in this thesis were performed.

I am indebted to the University of São Paulo (USP) and, in particular, to the Astronomy Department for supporting me as a graduate student. I very much appreciate the help from the secretaries and the IT team. I also thank the Physics \& Astronomy Department at George Mason University for supporting me as a visiting research scholar during the year 2007 and again from Nov-2008 to Feb-2009. I also would like to thank Rosemary Feijó and the staff at George Mason University, for helping me dealing with bureaucratic paperwork.

I am enormously grateful to my loving family for the constant support during good and bad times. I am so fortunate to have you! You are the most special gift that God 
gave me.

I thank so much my friends for making my life more pleasant: the always-present Takions' gang; my colleagues of the Dept. of Astronomy/USP, in special, my friends Marcio Avelar and Monica Uchida; all the friends I made in the US, in particular, Katie \& Chet, Maurizio, Rebekah, Yong, os meninos (Marcos, Rodrigo, Thiago, William, Daniel), as meninas (Kharis, Bruna, Andrea, Paula), and my partners in crime Cris Loesch and Katie Allen; my friends Charol, Guga, Subu, Marcelo Bianchi, Rodrigo Tchongo, Bruno, Gustavo Rojas, and Diego.

I specially would like to thank my friend and fiancé Zé Henrique for his love, support, and (sometimes irritating) optimism. My many many thanks for always being there for me. You make it easier when life gets hard.

Finally, I would like to express my gratitude to God, who has surrounded me with so many good things and wonderful people! 


\section{Contents}

Abstract $\quad$ ix

Resumo $\quad$ xi

Acknowledgments xiii

List of Figures $\quad$ xix

List of Tables $\quad$ xxv

1 Introduction 1

1.1 Stellar Winds From Low-Mass Stars . . . . . . . . . . . . . . . . . . . 3

1.1.1 The Sun as a Reference: The Solar Wind . . . . . . . . . . . . . . 3

1.1.2 Mass Loss in Solar-Like Stars . . . . . . . . . . . . . . 5

1.1.3 Mass Loss in T Tauri Stars . . . . . . . . . . . . . . . 7

1.2 Key Ingredients for Modeling Coronal Winds . . . . . . . . . . . . . . . . 8

1.2.1 Magnetic Fields . . . . . . . . . . . . . . . . 9

1.2.2 Rotation ........................ . . 10

1.3 Classical Solar Wind Models . . . . . . . . . . . . . . . . . . 14

1.3.1 The Parker Model . . . . . . . . . . . . . . . . . . . . . . . . . 14

1.3.2 The Weber-Davis Model . . . . . . . . . . . . . . . . . 16

1.3.3 The Pneumann-Kopp Model . . . . . . . . . . . . . . . . . . . . 19

1.4 Other Magnetized Wind Models . . . . . . . . . . . . . . . . . 19 
1.5 Motivation . . . . . . . . . . . . . . . . . 21

2 Magnetohydrodynamics $\quad 25$

2.1 General Characteristics of the Plasma . . . . . . . . . . . . . . 25

2.2 Derivation of the MHD Equations . . . . . . . . . . . . . 27

2.2.1 Preliminaries . . . . . . . . . . . . . . . . . . . . . 28

2.2.2 Some Useful Plasma Relations . . . . . . . . . . . . . . . . . . 30

2.2.3 The Generalized Ohm's Law . . . . . . . . . . . . . . . . . . . . 30

2.2 .4 The Induction Equation . . . . . . . . . . . . . . . . 32

2.2.5 The Equation of Motion for the Plasma . . . . . . . . . . . 33

2.2 .6 The Energy Equation . . . . . . . . . . . . . . . . . . . . 34

2.3 Final Remarks . . . . . . . . . . . . . . . . . . . . 36

$\begin{array}{lll}3 & \text { Our 3D Numerical Model } & 37\end{array}$

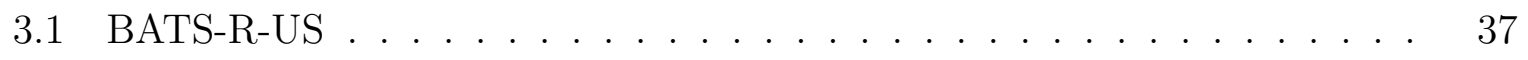

3.1.1 Normalized Units . . . . . . . . . . . . . . . . . . . . . . . . . 39

3.1.2 Solving the MHD Equations . . . . . . . . . . . . . . . 41

3.1.3 Applications of the Code . . . . . . . . . . . . . . . 43

3.2 Problem Setup . . . . . . . . . . . . . . . . . . . . . 43

3.2 .1 The Adopted Grid . . . . . . . . . . . . . . . 44

3.2 .2 The Initial Conditions . . . . . . . . . . . . . . . . . . 45

3.2.3 The Boundary Conditions . . . . . . . . . . . . . . 48

3.2.4 The Steady-State Solution . . . . . . . . . . . . . . . . . . . 49

4 3D Simulations of Magnetized Winds of Solar-Like Stars 51

4.1 The First Set of Simulations . . . . . . . . . . . . . . . . . . . 52

4.1.1 Evolution to Steady State . . . . . . . . . . . . 53

4.1.2 Steady-State Wind Profile . . . . . . . . . . . . . . . 56

4.1 .3 Meridional Flows . . . . . . . . . . . . . . . . . . . . . 60 
4.1 .4 The Neutral Point . . . . . . . . . . . . . . . . . 61

4.1 .5 Energetics . . . . . . . . . . . . . . . . 65

4.2 The Second Set of Simulations . . . . . . . . . . . . . . . . 67

4.2.1 Comparison Between First and Second Sets _. . . . . . . . . 67

4.3 The Third Set of Simulations with $\gamma=1.1 \ldots \ldots \ldots$. . . . . . 69

4.4 Discussion and Conclusion . . . . . . . . . . . . . . 71

5 Energetic Processes in Coronal Winds $\quad 73$

5.1 Mimicking Energetic Processes . . . . . . . . . . . . . . 73

5.1.1 Derivation of the Energy Equation . . . . . . . . . . . 74

5.1 .2 Numerical Model . . . . . . . . . . . . . . . . . . . . . 76

5.2 Results . . . . . . . . . . . . . . . . . . 77

5.2.1 Application: Estimates of X-ray Emission Measure . . . . . . . 80

5.3 Final Remarks . . . . . . . . . . . . . . . . . . . . 82

6 3D Simulations of Winds of $T$ Tauri Stars and The Influence of the $\begin{array}{ll}\text { Wind on Planet Migration } & 83\end{array}$

6.1 The Magnetic and Wind Structures . . . . . . . . . . . 85

6.2 Stellar Wind Effects on Planet Migration . . . . . . . . . . . . . . 91

6.3 Discussion and Conclusion . . . . . . . . . . . . . . . 96

7 3D Simulations of Misaligned Magnetospheres of WTTSs 99

7.1 The System Configuration . . . . . . . . . . . . . . . . . 99

7.2 Results. . . . . . . . . . . . . . . . . . . 102

7.2.1 Time-Dependent Behavior . . . . . . . . . . . . . . . . 102

7.2.2 The Effects of the Misalignment Angle . . . . . . . . . . . . . 102

7.3 Final Remarks . . . . . . . . . . . . . . . . . . . . 107

8 Summary of the Thesis and Perspectives 109 
$\begin{array}{ll}\text { A Refereed Publications } & 115\end{array}$

Bibliography

153

xviii 


\section{List of Figures}

1.1 Schematic picture of the mass being fed into the disk by the collapsing cloud, the mass from the disk being accreted by the star, and the ejection of a wind (values are for FU Ori objects). Picture credit: Hartmann and Kenyon (1996). . . . . . . . . . . . . . . . . . . . . 2

1.2 Solar wind speed as a function of heliographic latitude, measured by the Ulysses spacecraft. Picture credit: McComas et al. (1998). . . . . . . . . 4

1.3 Solar corona as viewed from the total solar eclipse of 1 August 2008, observed from Siberia. The coronal streamers and the coronal holes are easily seen in this picture and are related to zones of closed and open magnetic field lines, respectively. Picture credit: Pasachoff (2009). . . . . . . . .

1.4 Examples of short-lived structures on the Sun. A group of sunspots (upper left, image credit: $S O H O$ ); active regions, note also the magnetic loops (upper right, image credit: Stereo); eruptive prominence of the Sun (lower left, image credit: Stereo); coronal mass ejection (lower right, image credit:

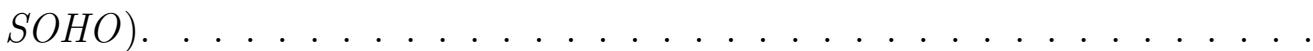

1.5 Mass-loss rates plotted versus X-ray fluxes: MS stars are represented by filled circles and evolved stars by open circles. Picture credit: Wood et al. (2005).

1.6 Schematic view of the suggested configuration of the young binary system V773 Tau A consisted of two WTTSs. Picture credit: adapted from Massi et al. (2008). . . . . . . . . . . . . . . . . . . 
1.7 The geometry of the coronal magnetic field of the CTTS V2129 Oph, as extrapolated from observed surface magnetograms with the assumption that the coronal field is force-free. The white lines depict closed magnetic field lines, while the blue lines represent open-field configuration. Picture credit: Jardine et al. (2008). . . . . . . . . . . . . . . . . . .

1.8 The distribution of periods for a sample of stars with masses $M_{\star} \gtrsim 0.25 \mathrm{M}_{\odot}$ (a) in NGC 2264 (age: 3 Myr) and (b) in the Orion Nebula Cluster (age: $\sim 1$ Myr). The vertical dotted lines are median of the rotation periods: (a) $4.7 \mathrm{~d}$ and (b) $6.75 \mathrm{~d}$. Picture credit: adapted from Lamm et al. (2005).

1.9 Theoretical curves of the rotational evolution of low-mass stars for different stellar masses. The models show the initial spin-up of the star followed by the longer phase of continuous spin-down. Picture credit: Landin et al.

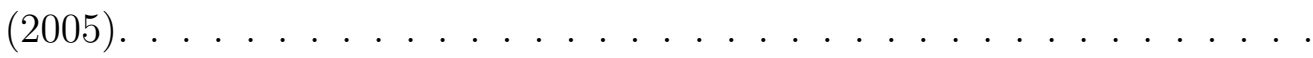

1.10 Velocity profiles of two thermally driven winds. Solid line: a Parker wind $(\gamma=1)$; dashed line: polytropic wind $(\gamma=1.05)$.

1.11 Example of the configuration of magnetic field lines in the Weber-Davis model, as looking at the equatorial plane. The star is in the center of the figure and rotates in the counterclockwise direction.

1.12 Magnetic field configuration self-consistently derived by an iterative method. Picture credit: Pneuman and Kopp (1971).

2.1 Orientation of the unit vectors $\hat{\mathbf{s}}$ and $\hat{\mathbf{n}}$, along and perpendicular to the field line, respectively; $\varpi_{c}$ is the local radius of curvature of the line.

3.1 The block-adaptive grid structure. In the figure, four different levels of refinement are shown.

3.2 Cartesian and spherical coordinate systems. . . . . . . . . . . .

3.3 Three different views of the grid. Inside each region, we indicate the maximum cell size of the region (in $r_{0}$ units). . . . . . . . . . . 46

3.4 The initial conditions adopted in the grid are composed of (a) a HD wind and (b) a dipolar magnetic field in vacuum. . . . . . . . . . . . . 
4.1 (a) and (b): Evolution of the magnetic field lines for S03. The color of each streamline represents a given iteration, ranging from $N=1(t=0$, blue) to 20,000 ( $t=7 \mathrm{~h}$, black). At $N=1$, the field is a dipole. The steady state is achieved at $N=148,000(t=51 \mathrm{~h})$. (c): Final steady-state configuration of the magnetic lines: (I) represents the zone of open field lines; (II) the zone of closed field lines; (III) the location of the neutral point. The thick line represents the position of the current sheet formed.

4.2 Three-dimensional view of the steady state for S03. . . . . . . . . . .

4.3 Meridional cuts of the steady-state configurations for simulations S01 to S05. Black streamlines represent the final configuration of the magnetic field. Contour plots of the velocity of the flow are shown in the background. The white line is the Alfvén surface. Continues in the next page. . . . . 57

4.3 Cont. . . . . . . . . . . . . . . . . . . . 58

4.4 Radial velocity of the wind as a function of $\theta$ at $r=4.5 r_{0}$, for S00 (double-dot-dashed line), S01 (solid line) and analytical solution of a nonmagnetized wind (dashed line). . . . . . . . . . . . . . . 59

4.5 Same as Fig. 4.4, but for S01 to S05, from $\theta=0^{\circ}$ to $\theta=90^{\circ} \ldots \ldots$

4.6 Meridional velocities $u_{\theta}$ for the steady-state configurations of simulations S01 to S05. Black streamlines are the magnetic field lines. The white line is the Alfvén surface. Last panel in the next page: The flux of matter in the $\theta$ direction, $\rho u_{\theta}$, for S01 to S05 at $r=4.5 r_{0}$. Continues in the next

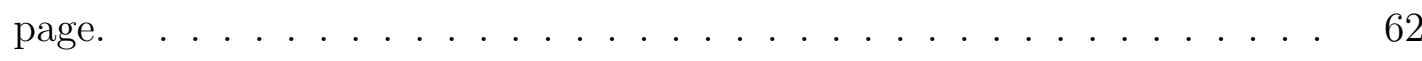

4.6 Cont. . . . . . . . . . . . . . . . . . . . 63

4.7 Trapping of particles inside the closed loop region for S03. Thick lines represent the magnetic field lines and vectors represent the flow velocity, both in the meridional plane $(y=0) \ldots \ldots . \ldots 64$

4.8 Temperature profile along the polar axis. . . . . . . . . . . . . 65

4.9 Cartoon of the energy flux q along a flux tube. . . . . . . . . . 67

4.10 Radial velocity profiles as a function of the distance along $\theta=45^{\circ}$ for the second set of simulations. . . . . . . . . . . . . . . . . . . . . . 68

4.11 Same as Fig. 4.3, but for simulations S01b and S02b, where $\gamma=1.1 . \quad$. $\quad 70$ 
5.1 (a) Radial dependence of the $\gamma$ adopted. (b) Initial radial velocity profiles. Solid line: constant $\gamma(\gamma=1.01)$; dashed line: variable $\gamma$ [Eq. (5.16)]. . . 78

5.2 Meridional cuts of the steady-state configurations for simulations V01, V02, S01, and S02. Black streamlines represent the final configuration of the magnetic field. Contour plots of the velocity of the flow are shown in the background. The white line is the Alfvén surface. . . . . . . . . . .

6.1 Meridional cuts of poloidal velocity profiles for: (a) case $\mathrm{E}\left(\beta_{0}=1\right)$, (b) case $\mathrm{A}\left(\beta_{0}=1 / 25\right)$, and $(\mathrm{c})$ case $\mathrm{F}\left(\beta_{0}=1 / 100\right)$. The black lines are streamlines of magnetic field and the white lines represent the Alfvén surface. . . . . . . . . . . . . . . . . . . .

6.2 Same as Fig. 6.1 for the remaining cases.

6.3 Three-dimensional view of the inner most region of case G. The lines plotted were selected as to outline the twisting of both closed and open magnetic lines anchored on the surface of the star. . . . . . . . . . . .

6.4 Comparison of: (a) the effective cross section of the planet that intercepts the stellar wind; (b) the wind ram pressure; and (c) the timescale for the stellar wind drag that significantly changes the planet's orbit. We use the same parameters as in cases A (red lines), E (blue lines), and F (green lines): solid lines are the results for our stellar wind model, and dashed lines for the WD model. . . . . . . . . . . . . . . . . . . . . .

6.5 Relative importance of the first (dashed lines) and second (dot-dashed lines) terms in the RHS of Eq. (6.4) calculated for case F using the results of our simulations (black) and using the WD model (red). Solid lines are the total ram pressures, i.e., the sum of first and second terms.

7.1 The magnetic field configuration in the simulations of misaligned magnetospheres: (a) in one stellar rotational period, the magnetic moment vector $\mathbf{m}$ draws an imaginary cone, whose central axis is the $z$-axis; (b) orientation of the magnetic field lines in the $y=0$ plane at the instant $t_{0}$.

7.2 Adopted grid in the simulations of misaligned magnetospheres. The numbers represent the maximum size of the cell inside each delimited region (in units of the stellar radius $r_{0}$ ) 
7.3 Temporal evolution of the simulation with $\theta_{t}=30^{\circ}$ during one full stellar rotational period. The panels show 7 different instants, starting from $t=$ $t_{1}$, and increasing in multiples of $1 / 6 P_{0}=4 \mathrm{~h}$, until $t=t_{1}+P_{0}$. We plot meridional cuts of the poloidal velocity, the magnetic field lines are represented by black lines, and the white line represents a contour where the magnetic field changes polarity (i.e., when $B_{r}=0$ ). The $x$-axis ranges from 0 to $10 r_{0}$ and the $y$-axis ranges from $-5 r_{0}$ to $5 r_{0} \ldots \ldots 103$

7.4 Meridional cuts of poloidal velocities for three misalignment angles $\theta_{t}=$ $10^{\circ}, 20^{\circ}$, and $30^{\circ}$. The panels are snapshots of the simulations after 8 stellar periods of rotation. The black lines represent magnetic streamlines and the white lines show contours for $B_{r}=0$ (when the magnetic field changes polarity). . . . . . . . . . . . . . . . . . . 104

7.5 Meridional cuts of the azimuthal components of velocity (left) and magnetic field (right) for three misalignment angles $\theta_{t}=10^{\circ}$ (upper row), $20^{\circ}$ (lower row), and $30^{\circ}$ (row in the next page). The instants plotted are the same as presented in Fig. 7.4 and the contours are in logarithmic scale. Continues in the next page. . . . . . . . . . . . . . . . . . 105

7.5 Cont. . . . . . . . . . . . . . . . . 106

7.6 Meridional cuts of azimuthal velocity of the wind, as seen from a larger portion of the grid. Contours are in logarithmic scale. . . . . . . . . . 106 


\section{List of Tables}

4.1 The complete set of simulations for solar-like stars. The columns represent, respectively: the name of the simulation, the temperature of the wind at the base of the corona, the density at $r_{0}$, the surface magnetic field intensity at the pole, the plasma- $\beta$ evaluated at the pole, and the value of $\gamma$ adopted. 53

4.2 Co-latitude dependence of the radial velocity $u_{r}$ at $r=4.5 r_{0}$. The columns represent, respectively: the name of the simulation, $u_{r}$ evaluated at $\theta=$ $18^{\circ}, u_{r}$ evaluated at $\theta=45^{\circ}$, and the difference $\Delta u_{r}=u_{r}\left(\theta=18^{\circ}\right)-u_{r}(\theta=$ $\left.45^{\circ}\right)$.

4.3 Mass-loss rate $\dot{m}$ per unit solid angle of the outflow. The columns represent, respectively: the name of the simulation, $\dot{m}$ evaluated at $\theta=0^{\circ}$, and $\dot{m}$ evaluated at $\theta=45^{\circ}$.

4.4 Location of the neutral point, $r_{N}$, along the equatorial plane as a function of $B_{0}$ for the first set of simulations.

4.5 Percentage amount of energy flux passing through four different crosssections at $2.2 r_{0}, 3 r_{0}, 4.2 r_{0}$, and $6 r_{0}$, along a flux tube for $\mathrm{S} 03$ and S05.

5.1 The set of simulations performed with a variable $\gamma$ [Eq. (5.16)]. The columns represent, respectively: the name of the simulation, the temperature and density at the coronal base, the magnetic field intensity at the pole, and the value of $\beta$ evaluated at the pole. 
5.2 Emission measure and X-ray luminosity generated inside the region of closed field lines for V01, V02, S01, and S02. The columns represent, respectively: the name of the simulation, the radial dependence of $\gamma$, the volume $V$ defined by the closed field lines, the estimated emission measure, the mean temperature inside $V$, and the estimated X-ray luminosity. . .

6.1 The set of simulations for WTTSs. The columns represent, respectively: the name of the simulation, the surface magnetic field intensity at the pole, the period of rotation of the star, $\gamma$, the density at $r_{0}$, and the plasma- $\beta$ evaluated at the pole. . . . . . . . . . . . . . . . 84 


\section{Chapter 1}

\section{Introduction}

Cellar mass loss is a phenomenon observed in practically the entire HertzsprungRussell diagram. Mass loss affects the evolution of the star from their birth to their death, therefore the knowledge of stellar mass-loss rates are essential to create realistic evolutionary models (Chiosi and Maeder 1986, Schaller et al. 1992). Intense ejections of mass are responsible for the creation of bright circumstellar nebulae observed around several stars (Chu et al. 1983, Sahai et al. 2007, Phillips and Ramos-Larios 2008). Even a tenuous stellar wind interacting with the interstellar medium can give rise to wind bubbles, being the heliosphere the most famous example (Wood and Churchwell 1989, Zurbuchen 2007). The ejecta from stars are also able to affect the chemical composition of galaxies (Portinari et al. 1998). Therefore, the study of stellar mass loss remains an active field of research. In this thesis, in particular, we model mass loss through stellar winds of low-mass stars.

Low-mass stars (with $M_{\star} \lesssim 2 \mathrm{M}_{\odot}$ ) are formed inside molecular clouds, specifically in dense condensations, known as cores (Alves et al. 2007, Goldsmith et al. 2008). When the internal pressures (e.g., thermal, magnetic, turbulent, etc.) of a core can not counterbalance the gravitational attraction, it collapses (Jeans 1902, Larson 1981, Goodwin et al. 2004). The final products of the collapse are stellar cores surrounded by rotating disks. These rapidly rotating disks will feed the stars for several million years, and will eventually form planetary systems (Pascucci et al. 2006, Brown et al. 2007).

Many observations have now probed the earliest phase of the life of a star, known as the pre-main-sequence (PMS) phase, and have shown that while material is being 


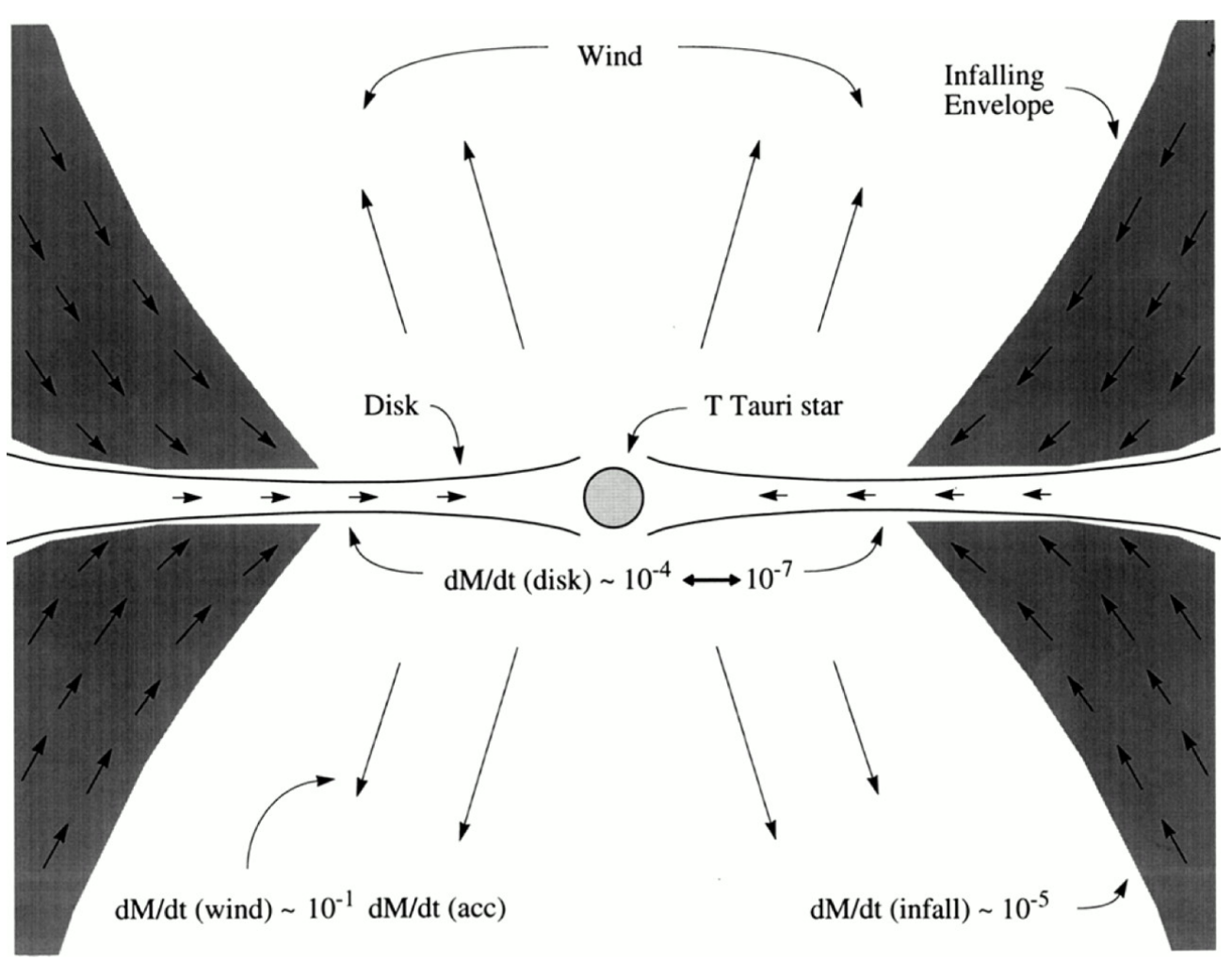

Figure 1.1: Schematic picture of the mass being fed into the disk by the collapsing cloud, the mass from the disk being accreted by the star, and the ejection of a wind (values are for FU Ori objects). Picture credit: Hartmann and Kenyon (1996).

accreted, the star is also losing mass through stellar winds and jets (Burrows et al. 1996, Zinnecker et al. 1998, Dutrey et al. 1998, Reipurth et al. 1999). Figure 1.1 presents a schematic picture of such scenario. In the phase where the star is surrounded by dense disks, observational findings show that the ratio between the rate of mass loss to the rate of accretion is $\sim 10 \%$ (Hartigan et al. 1995, Antoniucci et al. 2008). The accretion disks tend to dissipate as the star evolves (Hollenbach et al. 2000), but the stellar winds never cease.

The PMS phase leads the star to the main-sequence (MS) phase. In the MS, the star experiences nuclear fusion reactions of hydrogen into helium in its core (Iben 1967), while in the PMS phase, the main sources of energy are the contraction of the star and the nuclear deuterium burning (Hayashi and Nakano 1963). In the MS, the star continues to lose mass through stellar winds (Mullan et al. 1989; 1992, Wood et al. 2002; 2005). In particular, when the winds are magnetized, they also influence stellar angular momentum loss (Washimi and Shibata 1993, Holzwarth 2005). 
In the remainder of this chapter, we give an overview of stellar winds of low-mass stars, highlighting the main physical processes responsible for mass loss in such stars.

\subsection{Stellar Winds From Low-Mass Stars}

Our knowledge about the Sun, the solar wind, and the solar system can help us to better understand other low-mass stars and the effects that stellar winds have in their evolution and in their interaction with planetary systems and interstellar mediums. For this reason, studies of the solar corona have played a crucial role in understanding stellar winds in general.

\subsubsection{The Sun as a Reference: The Solar Wind}

The Sun is a MS star, with an estimated age of 4.7 billion years (Zolensky et al. 1999), effective temperature of $\sim 5800 \mathrm{~K}$ (Code 1973), and a hot corona with temperatures exceeding $10^{6} \mathrm{~K}$ (Feldman et al. 1999). Like almost all cool stars, the Sun is a magnetized star. The magnetic field that is observed at the surface is created through dynamo operation in the solar interior (Parker 1955, Babcock 1961), and is responsible for the cyclic activity of the Sun, among other observed phenomena (Baliunas and Vaughan 1985).

The solar magnetic field also influences the solar wind. It is believed that the coronal heating and the solar coronal wind acceleration are closely related (Grail et al. 1996). The heating of the solar corona is yet to be fully understood. Numerical models of the solar corona have shown that only thermal heating can not explain observations (Usmanov and Goldstein 2003). The explanation relies on solar magnetic fields, in part due to dissipation of magnetosonic waves that are generated in the convection zone (Osterbrock 1961). The mass-loss rate of the Sun measured in the equatorial plane is $\sim 2 \times 10^{-14} \mathrm{M}_{\odot} \mathrm{yr}^{-1}$ (Dupree 1986), which is a very tenuous mass loss when compared to the less evolved PMS stars (Hartigan et al. 1995) or the more evolved giant stars (Kemper et al. 2003, Lagadec et al. 2008). The solar wind has been explored in situ with satellites since the Mariner 2 in the 60's (Neugebauer and Snyder 1962). The spacecraft Ulysses measured the solar wind speed as a function of heliographic latitude and showed that it has slow and fast streams (McComas et al. 1998): the fast solar wind has velocities exceeding $700 \mathrm{~km} \mathrm{~s}^{-1}$, while 


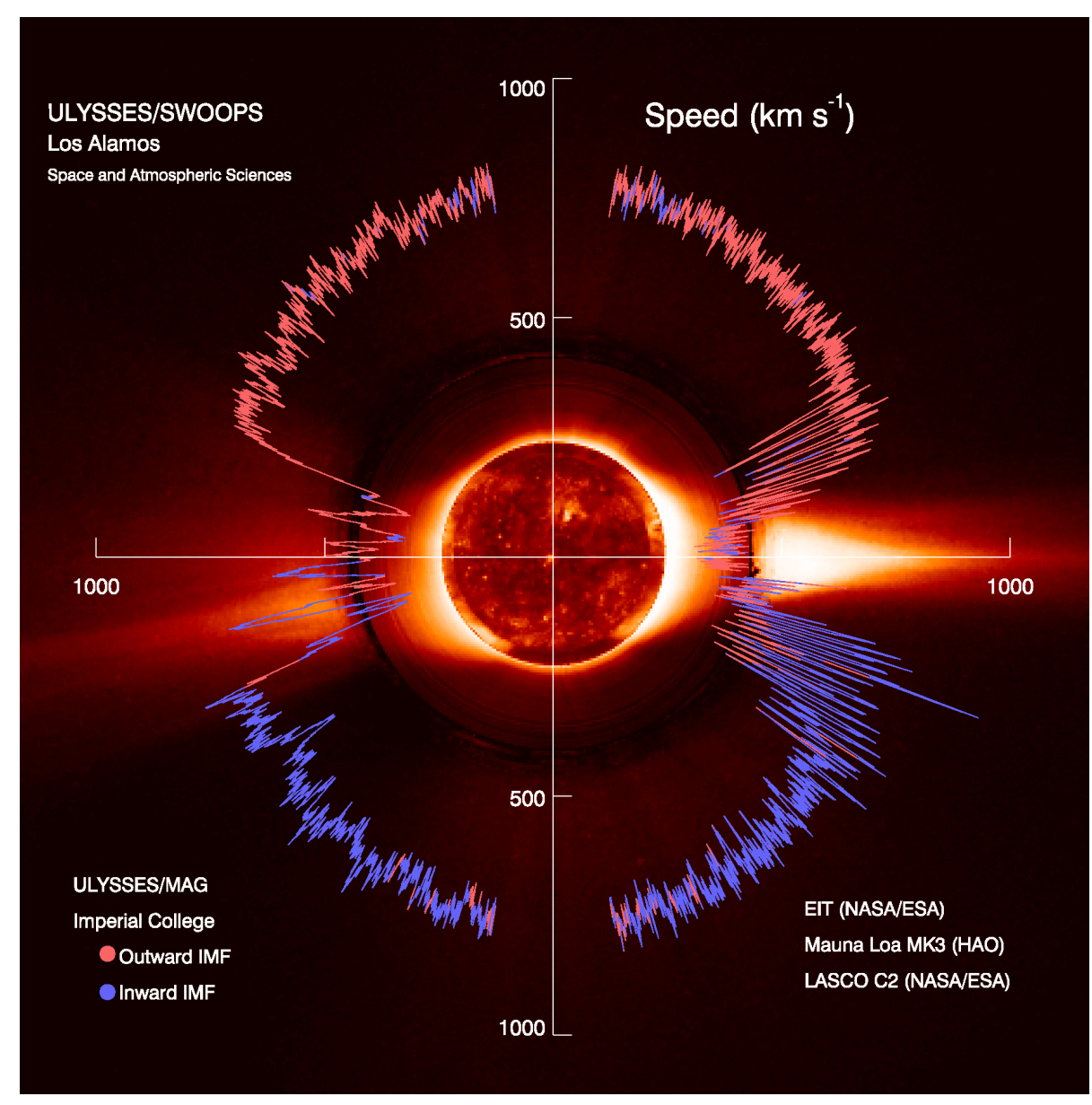

Figure 1.2: Solar wind speed as a function of heliographic latitude, measured by the Ulysses spacecraft. Picture credit: McComas et al. (1998).

the slow wind usually has less than $450 \mathrm{~km} \mathrm{~s}^{-1}$ (Fig. 1.2).

Because we are immersed in the solar wind, we understand in much more detail the physics operating in the Sun than we do in other stars. Recent sophisticated observations, e.g., via the $S O H O$ satellite and the spacecraft Ulysses (McComas et al. 1995, Suess and Smith 1996, Jones et al. 1998, Wilhelm 2006), have shown that the solar corona is a highly complex magnetic system. It consists of long-lived features (Fig. 1.3), such as streamers, associated to closed magnetic field lines, and coronal holes, associated to open magnetic field lines. The coronal holes are the source of the high speed solar wind ( $\mathrm{Tu}$ et al. 2005), while the slow wind is likely originating in the outer regions of the coronal streamers (Fisk and Zurbuchen 2006). Short-lived magnetic features are also present in the solar corona, such as the formation of sunspots and more energetic phenomena, as coronal mass ejections and solar flares (Fig. 1.4). 


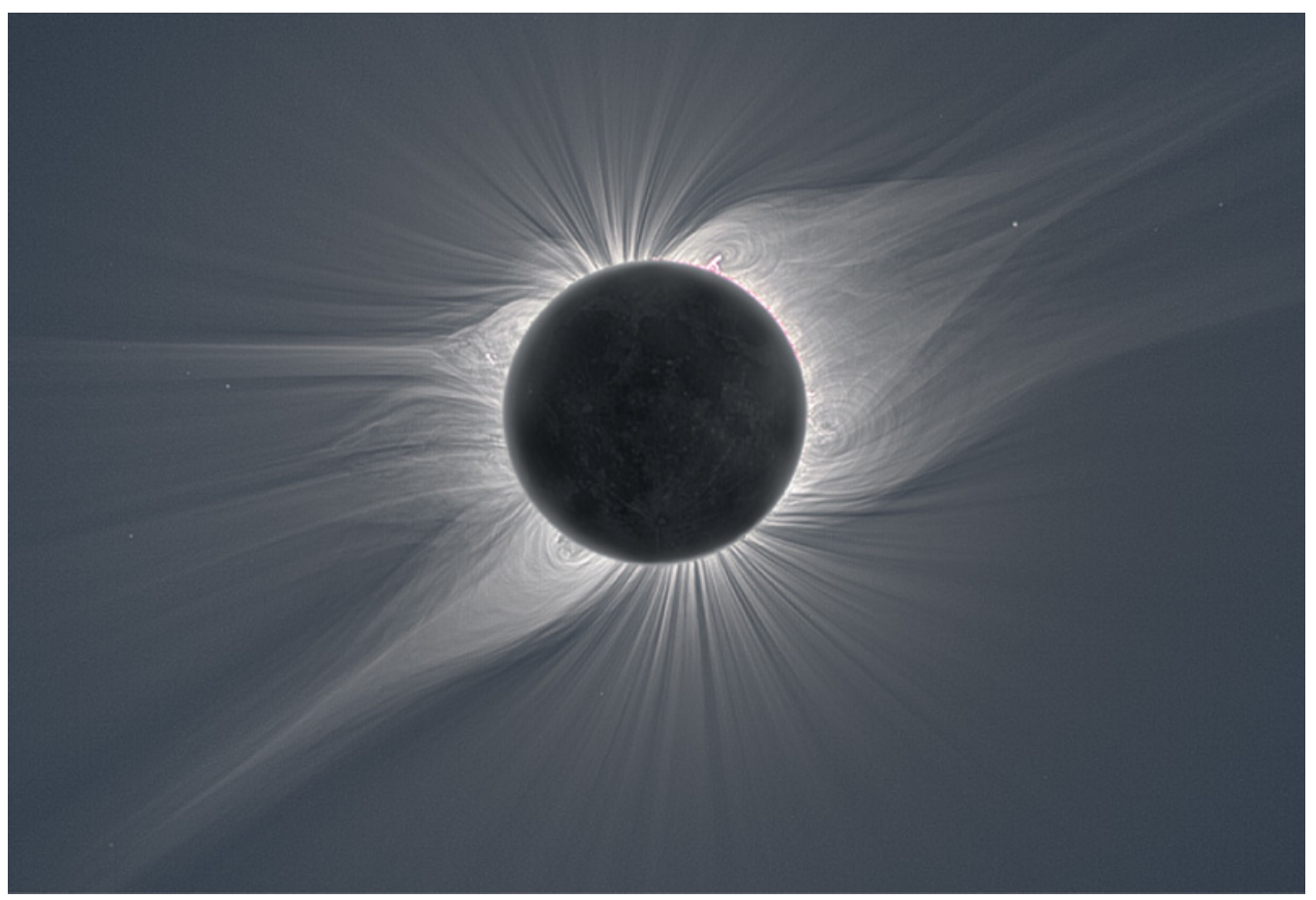

Figure 1.3: Solar corona as viewed from the total solar eclipse of 1 August 2008, observed from Siberia. The coronal streamers and the coronal holes are easily seen in this picture and are related to zones of closed and open magnetic field lines, respectively. Picture credit: Pasachoff (2009).

\subsubsection{Mass Loss in Solar-Like Stars}

Similar to the Sun, it is expected that any star with a chromosphere or a corona will harbor a wind. As the corona of the Sun can be probed by X-ray observations (Orlando et al. 2000, Peres et al. 2000), the detection of X-ray emission from other stars has been used to diagnose coronal emission (Telleschi et al. 2005, Scelsi et al. 2007). Indeed, X-ray observations have shown that the occurrence of coronae is very common among cool MS stars (Schmitt 1997), which make us believe that coronal winds will be present in solarlike stars. Unfortunately, details of the configuration of the coronal magnetic field and wind are only known for the Sun.

Direct measurements of coronal winds for stars other than the Sun are very difficult to obtain because, being the solar-like coronal winds very tenuous, spectral diagnostics as those used to study denser winds (e.g., P Cygni emission line profiles) are not present (Wood 2004). Although, indirect detections exist. Wood et al. (2002; 2005), for example, investigated the mass loss of solar-like stars based on the Ly- $\alpha$ absorption signature 

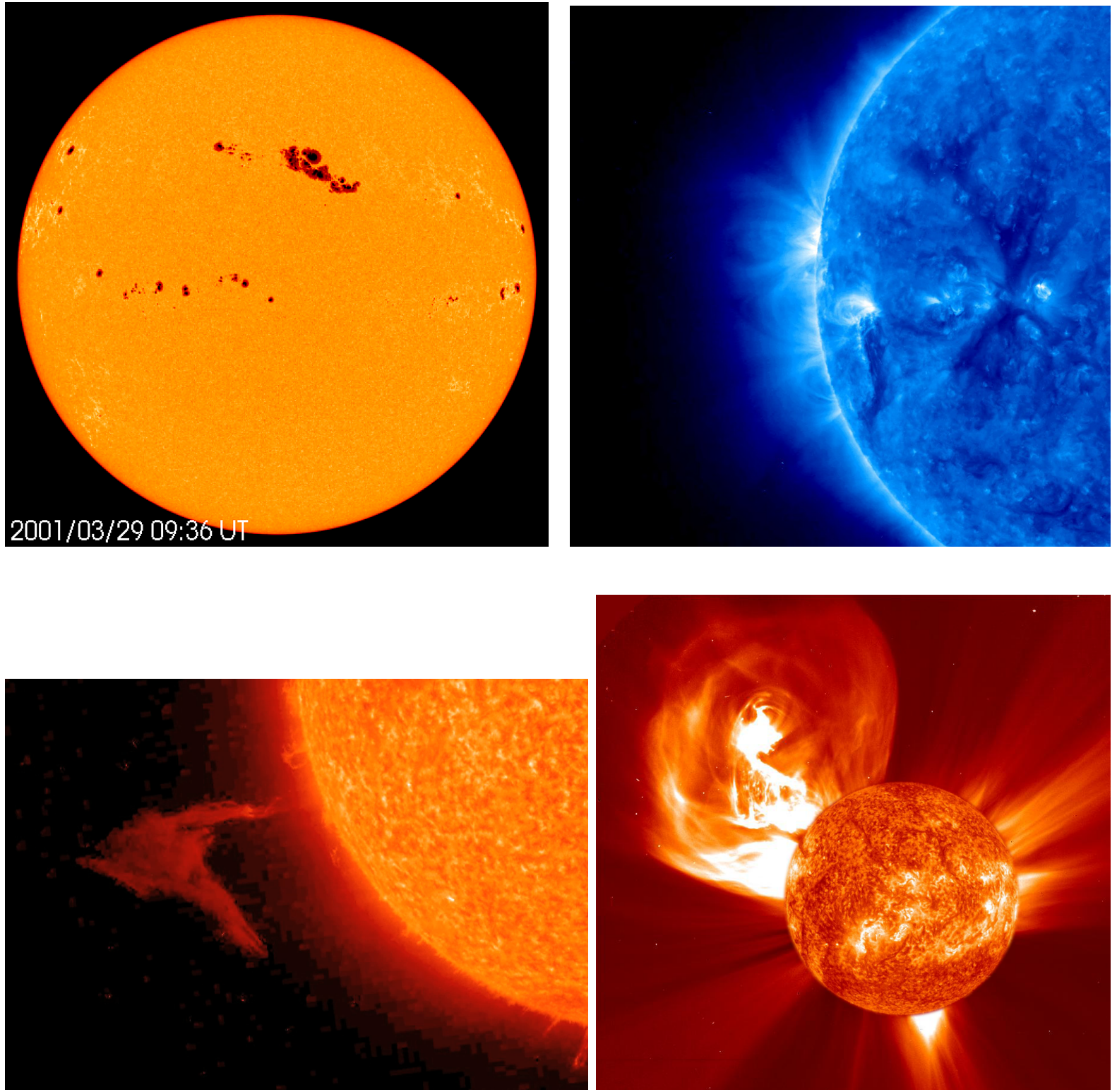

Figure 1.4: Examples of short-lived structures on the Sun. A group of sunspots (upper left, image credit: $\mathrm{SOHO}) ;$ active regions, note also the magnetic loops (upper right, image credit: Stereo); eruptive prominence of the Sun (lower left, image credit: Stereo); coronal mass ejection (lower right, image credit: SOHO).

originated from the collision of the stellar wind with the interstellar medium. This collision gives rise to an astrosphere, analogously to the heliosphere surrounding the Sun. As astrospheres do not exist in the absence of a stellar wind, the detection of astrospheric Ly- $\alpha$ absorption represents an indirect detection of solar-like stellar winds (Wood 2004).

As the intensity of the absorption is related to the strength of the wind, the absorption 


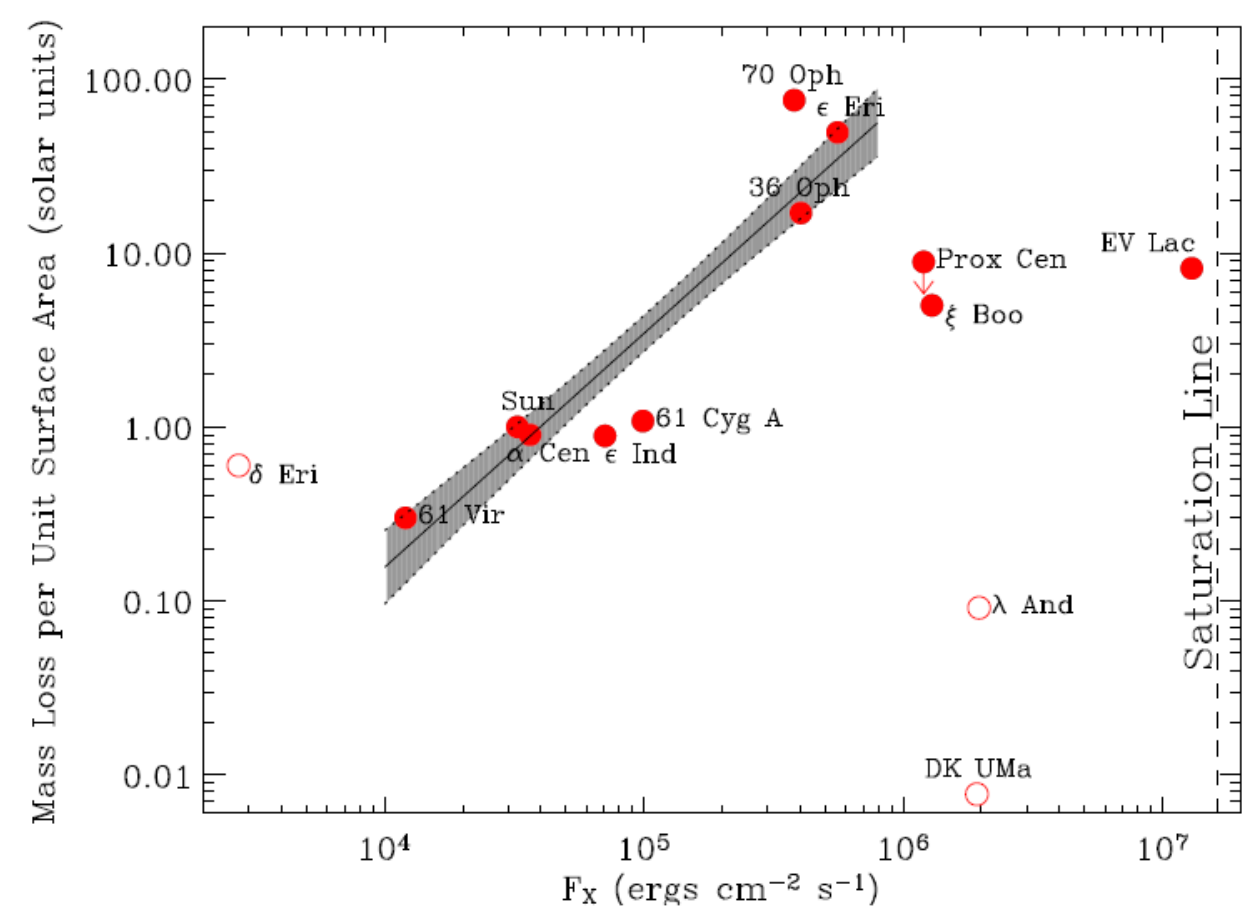

Figure 1.5: Mass-loss rates plotted versus X-ray fluxes: $M S$ stars are represented by filled circles and evolved stars by open circles. Picture credit: Wood et al. (2005).

provides an estimate of mass-loss rate. From the detected astrospheric absorptions and by assuming that the terminal velocities of coronal winds do not differ considerably from the solar value and that the interstellar medium around the stars does not vary greatly from one star to the next, Wood et al. (2002; 2005) obtained mass-loss rates for a sample of solar-like stars. They showed that the mass-loss rate $\dot{M}$ is correlated to the X-ray flux $F_{X}$ through a power-law fit $\left(\dot{M} \propto F_{X}^{1.34 \pm 0.18}\right)$, as shown in Fig. 1.5. This relation was then analyzed under the light of the rotation-activity and age-rotation relations derived from Ayres (1997), showing that the mass-loss rate decreases with the age $t$ of the star as $\dot{M} \propto t^{-2.00 \pm 0.52}$. This result led Wood et al. (2002; 2005) to suggest that the stellar wind of the young Sun might have been 1000 times more intense in the past than it is in the present Sun.

\subsubsection{Mass Loss in T Tauri Stars}

In the PMS phase, the Sun was a T Tauri star. T Tauri stars are recently formed lowmass stars $\left(0.5 \lesssim M_{\star} / M_{\odot} \lesssim 2\right)$, with spectral type ranging from $\mathrm{F}$ to $\mathrm{M}$, and radius of no 
more than 3 to $4 R_{\odot}$. These stars present higher mass-loss rates $\left(10^{-10}\right.$ to $10^{-8} \mathrm{M}_{\odot} \mathrm{yr}^{-1}$, Hartigan et al. 1995) than MS solar-like stars. Usually, T Tauri stars are classified in two categories: classical and weak-lined. A classical T Tauri star (CTTS) shows strong emission line activity, excess ultraviolet and infrared emissions, which are associated with accretion flows and the presence of circumstellar disks (Herbig 1962). A weak-lined T Tauri star (WTTS), conversely, has a weaker emission line activity and shows little or no trace of accretion, implying that the accretion disk has been dissipated (Wolk and Walter 1996). For this reason, they are also known as naked T Tauri stars (Walter et al. 1988).

CTTSs present signatures of both inflows (accretion from a circumstellar disk) and outflows (winds and jets), as evidenced through spectroscopic analysis. The mass loss is probed through the absorption in the blue side of $\mathrm{P}$ Cygni profiles of optically thick spectral lines, while the emission component of the $\mathrm{P}$ Cygni profile is believed to be due to the infall of the accreting material (Edwards et al. 2003). The exact launching region of the wind is uncertain, as there is an ongoing debate whether it originates from the star (Matt and Pudritz 2005) or from the disk (Shu et al. 1994, Koenigl and Pudritz 2000). Observational constraints on the wind launching region are thus critical for clarifying outflow origins (Gómez de Castro and Verdugo 2007, Kwan et al. 2007, Edwards 2009).

WTTSs do not show evidences for strong outflows as CTTSs do. Nevertheless, the presence of coronae is evidenced through the X-ray emission of a high temperature material (Preibisch et al. 2005). Scelsi et al. (2007) found the coronal plasma of CTTSs and WTTSs have similar characteristic temperatures. Over the last years, several observations, such as from the large X-ray surveys Chandra Orion Ultradeep Project (Getman et al. 2005) and XMM-Newton Extended Survey of the Taurus Molecular Cloud (Güdel et al. 2007), showed that the coronal temperature of T Tauri stars can exceed $10^{7} \mathrm{~K}$. It is believed that the bulk of the X-ray emission is coronal and comes from the material confined inside closed magnetic field structures.

\subsection{Key Ingredients for Modeling Coronal Winds}

The first key ingredient for the existence of a coronal wind is obviously the presence of high-temperature plasmas. However, as occurs in the solar corona, the magnetic field plays an important role in the acceleration and heating of coronal winds. Rotation is also an important ingredient in the mass and angular momentum losses of a star, as it is 
almost always correlated to the magnetic activity of the star.

\subsubsection{Magnetic Fields}

In the Sun, highly-detailed observations of the magnetic field, using both in situ and remote measurements, have shown a very complex magnetic configuration (e.g., McComas et al. 1995, Lin et al. 2005, Susino et al. 2008, see also §1.1.1). Although in less detail, magnetic activity has been detected in several other stars (e.g., Bagnulo et al. 2002, Reiners and Basri 2006, Phan-Bao et al. 2006, Wade et al. 2007, Hubrig et al. 2007, Valyavin et al. 2008, Donati et al. 2008b, Silvester et al. 2009), including stars similar to the Sun (Robinson et al. 1980, Petit et al. 2005; 2008) and T Tauri stars, where magnetic field strengths of a few $\mathrm{kG}$ have been detected (e.g., Johns-Krull et al. 1999, Valenti and Johns-Krull 2004, Johns-Krull 2007, Donati et al. 2007, Yang et al. 2008).

With the improvement and development of observational techniques, the detection of a more complex magnetic field geometry in young stars has just started to be accomplished, as for example in the CTTSs V2129 Oph (Donati et al. 2007), BP Tau (Donati et al. 2008a), and CV Cha \& CR Cha (Hussain et al. 2009), where surface magnetic maps have been derived from spectropolarimetric data.

Magnetic activity in T Tauri stars is usually explained as a scaled-up magnetic activity similar to the one observed in the Sun (Preibisch et al. 2005). V773 Tau A, a young binary system consisted of two WTTSs, is known to posses high levels of magnetic activity (Phillips et al. 1991; 1996). Recently, Massi et al. (2008) observed two extended radio emissions that were interpreted as associated to the primary and secondary stars of the system. They suggested that these features were produced by helmet streamer structures from each of the stars (Fig. 1.6), that when overlapped at certain orbital phases, would result in reconnection producing the giant flares that were observed: radio emission would increase from a few mJy at apastron to more than $100 \mathrm{mJy}$ at periastron. In another recent work, Skelly et al. (2008) reported the findings of slingshot prominences on TWA6, another WTTS.

In the literature, it is often adopted that $\mathrm{T}$ Tauri stars present strong dipolar magnetic fields, such as in magnetospheric accretion models for CTTSs (Koenigl 1991, Hartmann et al. 1994). However, the complex observed magnetic topology, specially close to the star, is inconsistent with the assumption of dipolar stellar magnetic field (Safier 1998). 


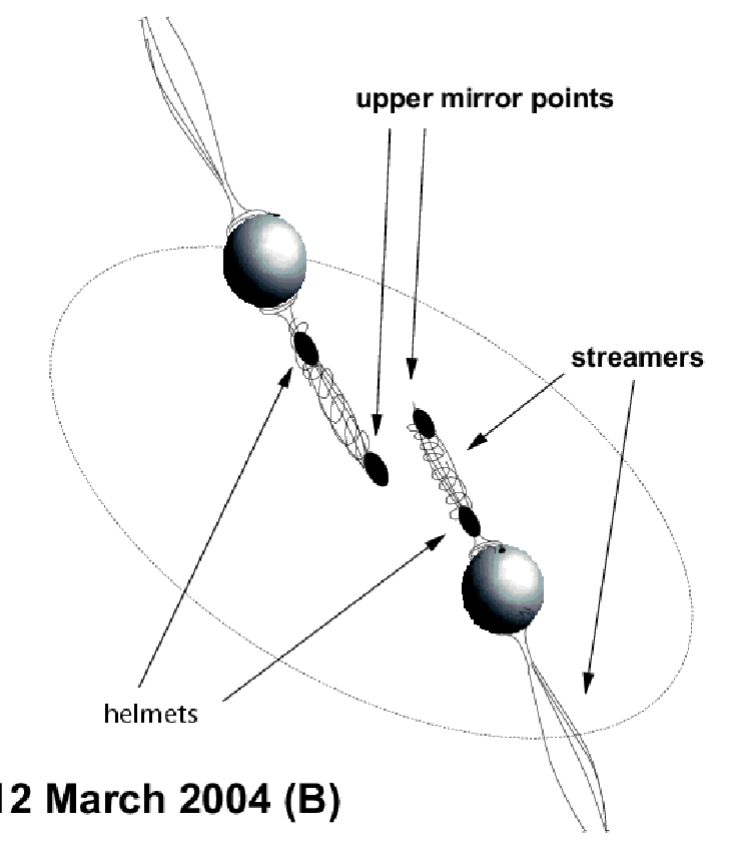

Figure 1.6: Schematic view of the suggested configuration of the young binary system V7r3 Tau A consisted of two WTTSs. Picture credit: adapted from Massi et al. (2008).

For example, magnetic field extrapolations from surface magnetograms have shown that the geometry of the large-scale field of $\mathrm{T}$ Tauri stars may be very complex (Jardine et al. 2008, Gregory et al. 2008), as illustrated in Fig. 1.7. However, it is believed that at large distances from the star, the multipolar components with high orders become negligible, in a way that the dipolar component should become the dominant one (Valenti and Johns-Krull 2004).

\subsubsection{Rotation}

Observations have shown that young stars present a bimodal distribution of rotational periods (Bouvier et al. 1993, Choi and Herbst 1996, Herbst et al. 2002, Rebull et al. 2006). In samples of PMS stars from the Orion Nebula Cluster and NGC 2264, Cieza and Baliber (2007) found that stars with disks possess a distribution of rotational periods peaking around $P_{0} \sim 8 \mathrm{~d}$, while stars without disks present a peak at $P_{0} \sim 2 \mathrm{~d}$. Figure 1.8, adapted from the work of Lamm et al. (2005), illustrates such bimodal distribution for a sample of low-mass stars $\left(M_{\star} \gtrsim 0.25 \mathrm{M}_{\odot}\right)$ in NGC 2264 and in the Orion Nebula Cluster. These facts led to the suggestion that the accretion disk is somehow regulating the spin 


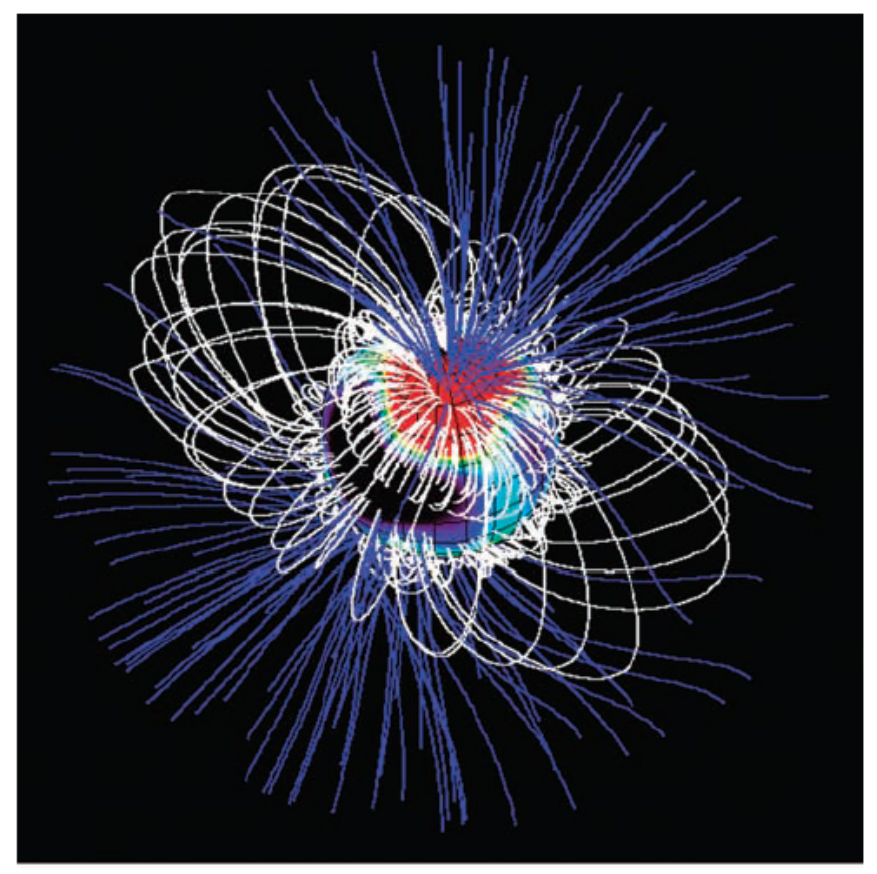

Figure 1.7: The geometry of the coronal magnetic field of the CTTS V2129 Oph, as extrapolated from observed surface magnetograms with the assumption that the coronal field is force-free. The white lines depict closed magnetic field lines, while the blue lines represent open-field configuration. Picture credit: Jardine et al. (2008).

of the star during the PMS (explaining the second peak in the histograms of Fig 1.8) and that, with the dissipation of the accretion disk, the star is free to spin up as it contracts towards the MS (explaining the first peak in the histograms of Fig 1.8).

The material in the disk rotates with velocities that are approximately Keplerian, possessing a high specific angular momentum. Hence, it would be expected that while this material is accreted, the angular momentum of the star would increase, spinning up the star (Hartmann and Stauffer 1989). However, a CTTS is observed to rotate with a period that is approximately constant in the first few Myr of its life (Rebull et al. 2006).

Several mechanisms have been proposed to account for such spin regulation. A possible mechanism is the disk-locking, in which the stellar magnetic field that is connected to the disk transfers angular momentum from the star to the disk, while matter is being transferred from the disk to the star (Ghosh and Lamb 1979, Koenigl 1991). The disk would then remove the acquired angular momentum through a wind, for example a diskwind (for a review, see Koenigl and Pudritz 2000) or an X-wind (Shu et al. 1994). There are however problems with the disk-locking mechanism (see Matt and Pudritz 2008c), 
(a) NGC 2264

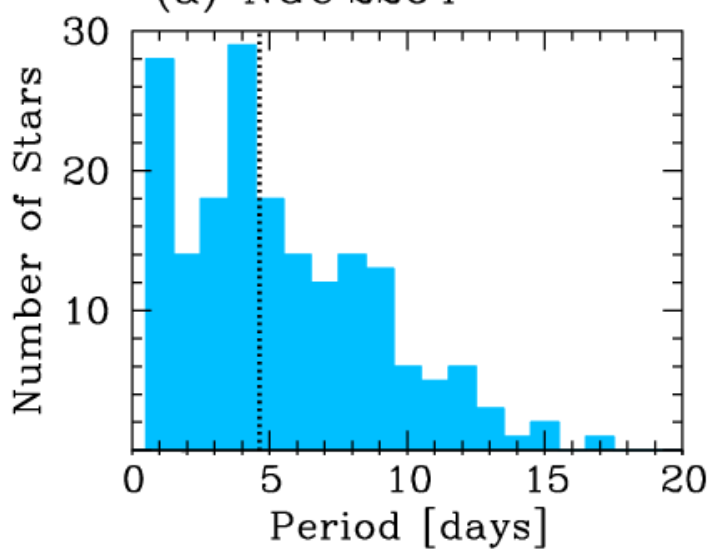

(b) ONC

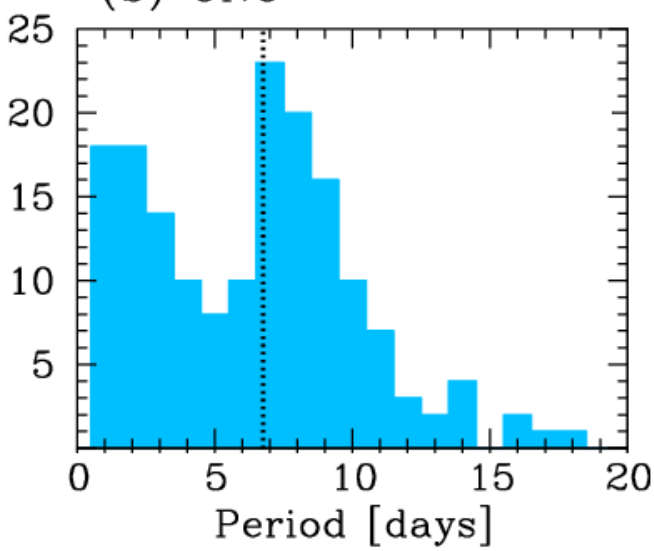

Figure 1.8: The distribution of periods for a sample of stars with masses $M_{\star} \gtrsim 0.25 M_{\odot}(a)$ in NGC 2264 (age: 3 Myr) and (b) in the Orion Nebula Cluster (age: 1 Myr). The vertical dotted lines are median of the rotation periods: (a) $4.7 d$ and (b) $6.75 d$. Picture credit: adapted from Lamm et al. (2005).

which led Matt and Pudritz (2005; 2008a;b) to suggest another mechanism that could prevent the spin-up of the star by means of a magnetized stellar wind. As shown by Matt and Pudritz (2005), the accretion-powered stellar wind may provide the necessary removal of angular momentum if the mass-loss of the wind is at least $10 \%$ of the mass-accretion rate.

When the star finally reaches the MS phase (with ages of $\sim 100 \mathrm{Myr}$ ), its contraction practically halts. However, it has long been inferred that solar-like stars present a decline in the rotational velocities with advancing age (Kraft 1967). Skumanich (1972) empirically determined that the rotational period of a solar-like star in the MS changes accordingly to its age obeying the relation $P_{0} \propto t^{1 / 2}$. For these reasons, it is believed that a rotation braking mechanism is also operating during the MS. The main candidate that can provide the rotational braking of the star is the stellar wind, but instead of the accretion-powered stellar wind, as suggested during the early phases of classical T Tauri, a rotating magnetically coupled stellar wind has to be evoked (Mestel 1968, Belcher and MacGregor 1976, Kawaler 1988).

Qualitatively, the mechanism acts as follows. In the case the star has a sufficiently strong magnetic field, the magnetic field try to bring the surrounding wind, a highly conducting gas that is coupled to the field, into corotation with the star (Mestel 1968). 
Thus, the wind feels a torque after leaving the stellar surface, carrying angular momentum from the star. If there is a continuous flow of matter leaving the star, there will be a continuous angular momentum loss that may efficiently spin down the star. Several works have shown that the necessary amount of angular momentum could have been lost through the magnetized wind during the life of the Sun in the MS phase (e.g., Belcher and MacGregor 1976, Washimi and Shibata 1993), indicating that the magnetic braking through the action of stellar winds may be an efficient way to lose angular momentum.

Figure 1.9, from Landin et al. (2005), illustrates the discussion of this section. It presents the rotational evolution of a low-mass star, derived from numerical modeling, showing the spun up of the star during the contraction phase in the PMS, followed by the slowly spun down during the MS.

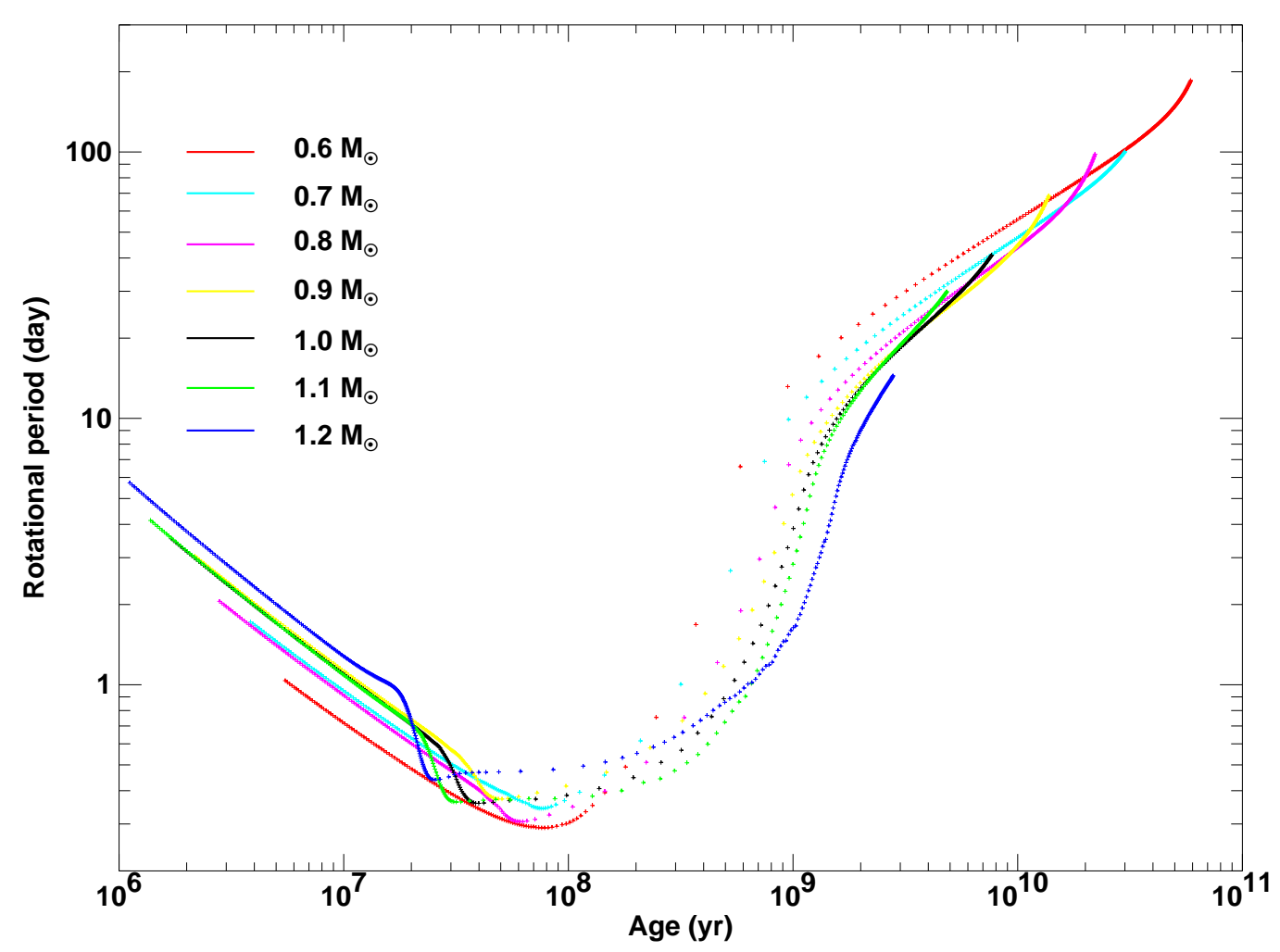

Figure 1.9: Theoretical curves of the rotational evolution of low-mass stars for different stellar masses. The models show the initial spin-up of the star followed by the longer phase of continuous spin-down. Picture credit: Landin et al. (2005). 


\subsection{Classical Solar Wind Models}

\subsubsection{The Parker Model}

Parker (1958) developed a model for the solar wind to explain the tenuous mass loss of the Sun. In this model, the solar wind is considered as an expanding bubble driven by the thermal pressure difference between the heliosphere and the interstellar medium. The Parker model, also known as thermally driven model, is one-dimensional (1D), isothermal, and purely hydrodynamical, i.e., the magnetic field is not taken into account. In the absence of magnetic forces and rotation, the equilibrium between the gradient of the thermal pressure, the gravitational force, and the wind inertial forces describes the driving mechanism of a wind thermally accelerated ${ }^{1}$ :

$$
\begin{aligned}
\frac{\mathrm{D} \mathbf{u}}{\mathrm{D} t} & =-\frac{\nabla p}{\rho}+\mathbf{g}, \\
\frac{\partial \mathbf{u}}{\partial t}+(\mathbf{u} \cdot \nabla) \mathbf{u} & =-\frac{\nabla p}{\rho}+\mathbf{g},
\end{aligned}
$$

where $\mathbf{u}, \rho$, and $p$ are the velocity, density, and pressure of the wind, respectively, $t$ is the time, and $\mathbf{g}$ is the gravitational acceleration. In the last equation, all forces are directed along the radial coordinate $r$ and in steady state $(\partial / \partial t=0)$, it is rewritten as

$$
u_{r} \frac{\partial u_{r}}{\partial r}=-\frac{1}{\rho} \frac{\partial p}{\partial r}+g_{r}
$$

where the index " $r$ " stands for radial component of the variable and $g_{r}=-G M_{\star} / r^{2}$. From the conservation of flux of matter ${ }^{2}$, the mass-loss rate is a constant of the wind and for a spherically symmetric wind it is given by

$$
\dot{M}=4 \pi \rho r^{2} u_{r}
$$

Considering ideal gas, the pressure is set as $p=\rho k_{B} T /\left(\mu m_{p}\right)$, where $k_{B}$ is the Boltzmann constant, $T$ the temperature, and $\mu m_{p}$ is the mean mass of the particle. Equations (1.3) and (1.4) give

$$
\frac{1}{u_{r}} \frac{\partial u_{r}}{\partial r}=\left[\frac{2 a^{2}}{r}-\frac{G M_{\star}}{r^{2}}\right] /\left(u_{r}^{2}-a^{2}\right),
$$

\footnotetext{
${ }^{1}$ The operator $D / D t=\partial / \partial t+\mathbf{u} \cdot \nabla$ is the total derivative that will be introduced in $\S 2.2 .1$. For now, it suffices to say that $D \mathbf{u} / D t$ represents the acceleration of the fluid.

${ }^{2}$ Chapter 2 formally introduces the equations of the (magneto)hydrodynamics.
} 


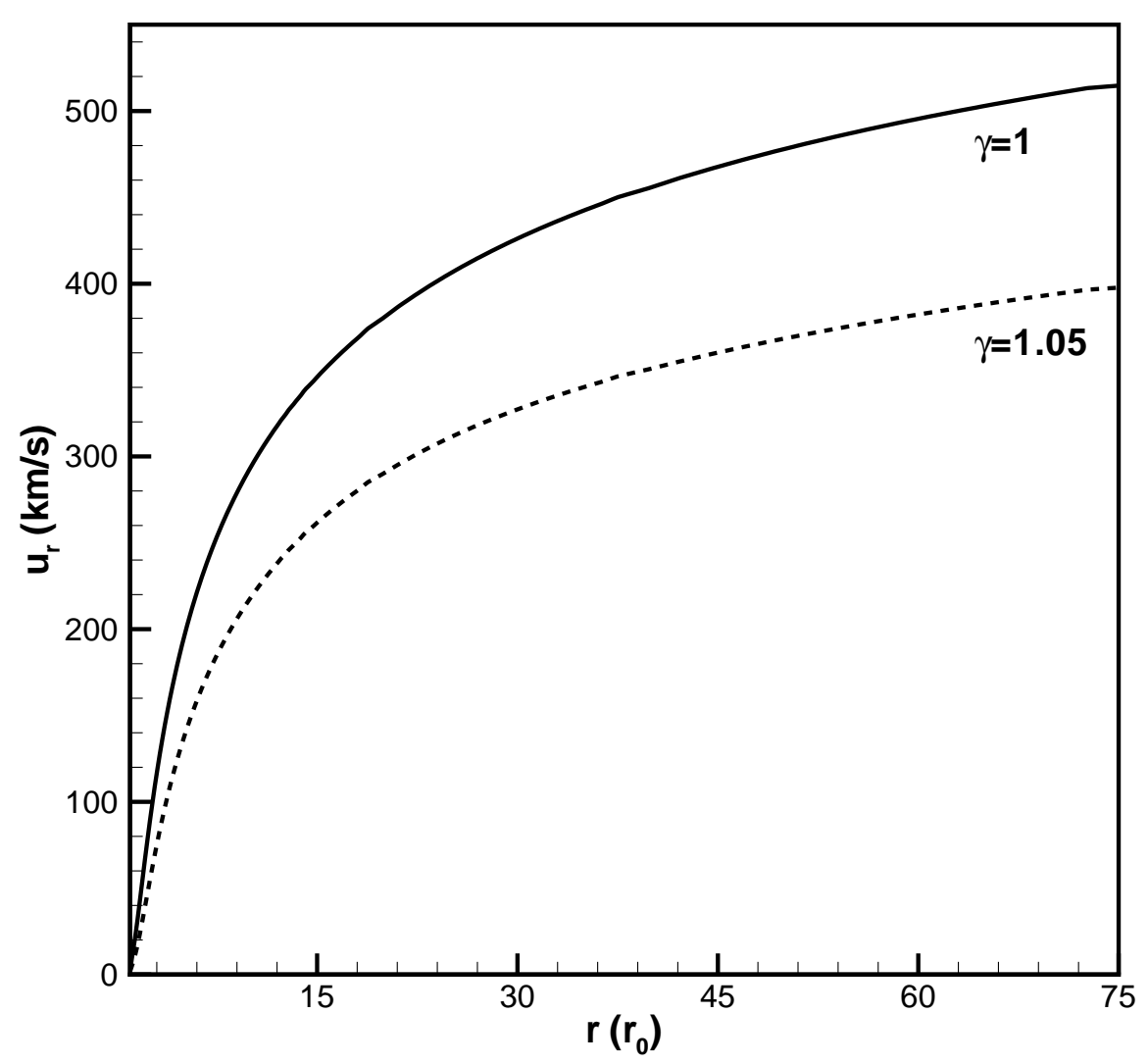

Figure 1.10: Velocity profiles of two thermally driven winds. Solid line: a Parker wind $(\gamma=$ $1)$; dashed line: polytropic wind $(\gamma=1.05)$.

where $a=\sqrt{p / \rho}=\sqrt{k_{B} T /\left(\mu m_{p}\right)}$ is the isothermal sound velocity. There is only one physical solution for $u_{r}$ : the solution that crosses the critical point with positive derivative $\left(\partial u_{r} / \partial r>0\right.$, i.e., accelerating). Equation (1.5) is then integrated to obtain the velocity of the wind, its density and pressure profiles, and hence its mass-loss rate. Assuming a star with mass $M_{\star}=1 M_{\odot}$ and radius $r_{0}=1 R_{\odot}$, and a wind with temperature $T_{0} \simeq 1.5 \mathrm{MK}$ and $\mu=0.5$ (totally ionized wind), the solution of the velocity is the solid line presented in Fig. 1.10.

For a given velocity profile, the wind density profile is derived from the equation of mass conservation [Eq. (1.4)]

$$
\rho=\frac{\dot{M}}{4 \pi r^{2}}=\rho_{0} \frac{u_{r, 0} r_{0}^{2}}{u_{r} r^{2}},
$$

where the subscript "0" indicates that the variable is evaluated at the base of the wind. It is interesting to note that the Parker wind does not depend on the density neither on the pressure of the wind, instead it depends on the temperature, through the sound 
velocity $a$ [Eq. (1.5)]. Assuming $\rho_{0} \simeq 1.5 \times 10^{-16} \mathrm{~g} \mathrm{~cm}^{-3}$ at the coronal base, a mass-loss rate of $\simeq 8 \times 10^{-14} \mathrm{M}_{\odot} \mathrm{yr}^{-1}$ is found, which is similar to what is observed in the Sun.

The thermally driven wind can also describe the more general case of a polytropic corona. In the polytropic wind, the equation of state is given by $p \propto \rho^{\gamma} \propto T^{\gamma /(\gamma-1)}$. In fact, the isothermal case is a particularity of the polytropic case where $\gamma=1$. In a polytropic wind, it is easy to show that Eq. (1.5) is written as

$$
\frac{1}{u_{r}} \frac{\partial u_{r}}{\partial r}=\left[\frac{2 \gamma a^{2}}{r}-\frac{G M_{\star}}{r^{2}}\right] /\left(u_{r}^{2}-\gamma a^{2}\right) .
$$

Figure 1.10 also presents the velocity profile for the case when $\gamma=1.05$ (dashed line). An increase in $\gamma$ results in lower velocities. As will be discussed in Chap. 5, $\gamma$ is related to the energy input in the wind and $\gamma=1$ represents the case of maximum input of energy, where the wind is kept isothermal in spite of its expansion.

\subsubsection{The Weber-Davis Model}

Another classical model developed for the solar wind was created by Weber and Davis (1967). Different from the Parker model, the Weber-Davis model includes rotation and magnetism. In the Parker model, the solution is spherically symmetric, i.e., valid for any colatitude $\theta$. In the Weber-Davis model, the solution is only valid on the equatorial plane $\left(\theta=90^{\circ}\right)$.

In the presence of a radial outflow (no rotation), a radial magnetic field is not deformed, as from the magnetic induction equation,

$$
\frac{\partial \mathbf{B}}{\partial t}=\nabla \times(\mathbf{u} \times \mathbf{B})=\nabla \times\left[u_{r} B_{r}(\hat{\mathbf{r}} \times \hat{\mathbf{r}})\right]=0 .
$$

Also, if $\mathbf{B} \| \mathbf{u}$, there is no magnetic force in the wind (the Lorentz force is proportional to $\left.\mathbf{u} \times \mathbf{B}=u_{r} B_{r}(\hat{\mathbf{r}} \times \hat{\mathbf{r}})=0\right)$. Hence, a purely radial magnetic field immersed in a wind with a purely radial velocity field leads to an outflow with the same characteristics of a non-magnetized outflow. In the Weber-Davis model, the Sun is assumed to have a radial magnetic field and a solid body rotation. In this case, when the star is rotating, the stellar wind leaves the star with an azimuthal component of the velocity, which then creates an azimuthal magnetic field. Hence, due to rotation, the radial magnetic field is distorted, giving rise to a spiral configuration of magnetic field lines. Figure 1.11 illustrates such configuration, for the case when the rotation is given in the counterclockwise direction. 


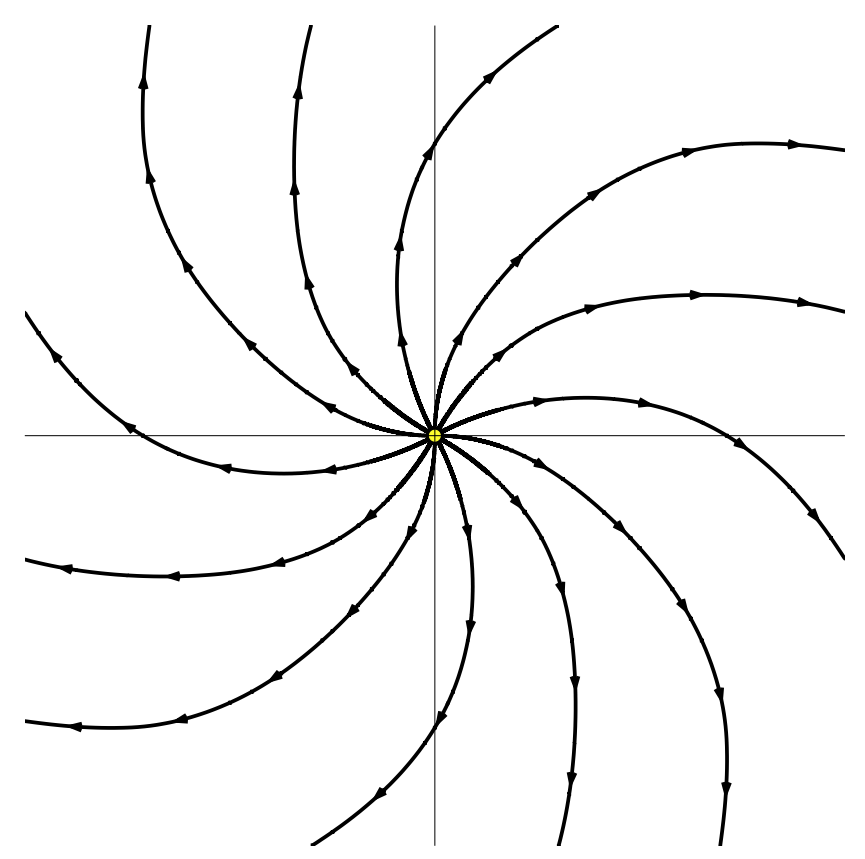

Figure 1.11: Example of the configuration of magnetic field lines in the Weber-Davis model, as looking at the equatorial plane. The star is in the center of the figure and rotates in the counterclockwise direction.

The Sun is a slow rotator with a period of $\sim 28$ days, or an angular velocity of $\sim 2.6 \times$ $10^{-6} \mathrm{~s}^{-1}$. Despite its slow rotation, Weber and Davis (1967) showed that the magnetic field acquires an azimuthal component, that becomes progressively more important for larger distances from the star. The theory of the Weber-Davis model is simple and elegant. The velocity of the wind is written as

$$
\mathbf{u}=u_{r} \hat{\mathbf{r}}+u_{\varphi} \hat{\varphi}
$$

where $\varphi$ is the azimuthal angle. Analogously, the magnetic field is

$$
\mathbf{B}=B_{r} \hat{\mathbf{r}}+B_{\varphi} \hat{\varphi}
$$

As in the Parker model, the Weber-Davis model is constructed under steady-state conditions. So from the magnetic induction equation,

$$
\nabla \times(\mathbf{u} \times \mathbf{B})=\frac{1}{r} \frac{\partial}{\partial r}\left[r\left(u_{r} B_{\varphi}-u_{\varphi} B_{r}\right)\right]=0 .
$$

The previous equation is integrated and the constant of integration is evaluated at the base of the wind: because the azimuthal velocity is much larger than the radial velocity 
and because the magnetic field lines leave the stellar surface approximately radially, at $r_{0}, u_{r, 0} B_{\varphi, 0} \ll u_{\varphi, 0} B_{r, 0}$. Thus

$$
r\left(u_{r} B_{\varphi}-u_{\varphi} B_{r}\right)=\text { const. }=-r_{0}^{2} \Omega B_{r, 0},
$$

where $\Omega$ is the angular velocity of the star. Furthermore, since $\nabla \cdot \mathbf{B}=0$

$$
r^{2} B_{r}=\text { const. }=r_{0}^{2} B_{r, 0} .
$$

From the last two equations, we find

$$
\frac{B_{\varphi}}{B_{r}}=\frac{u_{\varphi}-r \Omega}{u_{r}}
$$

Another constant of the Weber-Davis model is found from the azimuthal component of the wind momentum equation, which has only the terms relative to the magnetic force

$$
\begin{aligned}
& \frac{\mathrm{D}}{\mathrm{D} t}\left(u_{\varphi} \hat{\varphi}\right)=\frac{[(\nabla \times \mathbf{B}) \times \mathbf{B}]_{\varphi}}{4 \pi \rho}, \\
& \frac{u_{r}}{r} \frac{\partial\left(r u_{\varphi}\right)}{\partial r}=\frac{B_{r}}{4 \pi \rho r} \frac{\partial\left(r B_{\varphi}\right)}{\partial r} .
\end{aligned}
$$

Using the mass conservation [Eq. (1.4)], we integrate last equation to give

$$
l=r u_{\varphi}-\frac{r B_{\varphi} B_{r}}{4 \pi \rho u_{r}}=\text { const. }
$$

which we recognize as being the angular momentum per unit mass $l$. Equation (1.17) shows that the specific angular momentum of the wind is carried by the rotating fluid (the usual specific angular momentum of matter $r u_{\varphi}$ ) and by the magnetic field. It can be shown that the constant of integration is $l=\Omega r_{A}^{2}$, where $r_{A}$ is the Alfvén radius, where the velocity of the wind equals the Alfvén velocity

$$
v_{A}=\frac{B}{(4 \pi \rho)^{1 / 2}} .
$$

Since the term $r u_{\varphi}=r^{2} \Omega$ corresponds to matter corotating with the star, the constant $l=\Omega r_{A}^{2}$ means that the magnetized wind carries angular momentum from the star, behaving as if the star rotates as a solid body out to $r_{A}$.

In Weber and Davis (1967), the equations derived before, together with the radial component of the momentum equation, are integrated to find the velocity and magnetic field profiles of the solar wind. They found that (i) the radial velocity resembles the Parker solution (as in Fig. 1.10), (ii) the magnetic field configuration has a spiral pattern (Fig. 1.11), (iii) due to conservation of angular momentum, the azimuthal velocity profile increases near the star (representing matter corotating with the star) and decreases for large distances. 


\subsubsection{The Pneumann-Kopp Model}

Pictures from the solar eclipse show that close to the Sun, there are regions of closed structures (see Fig. 1.3). These regions are dominated by strong magnetic fields, where the coronal gas can be trapped. To explain such structures, another model for the solar wind was developed by Pneuman and Kopp (1971). Although rotation is not considered in this model, it is a two-dimensional model, where the magnetic field has radial and latitudinal components. By means of a numerical iterative method, Pneuman and Kopp (1971) constructed a model for the magnetized solar wind where the structure of the wind is determined through the interaction of gravitational, pressure, inertial and magnetic forces. This iterative method consists of three steps: first, the magnetic field is reconstructed from a given distribution of currents $\mathbf{J}$, since $\mathbf{J} \propto \nabla \times \mathbf{B}$; second, given the magnetic field geometry, the solar wind equations are solved along each individual line resulting in density and velocity distributions; third, from the density and velocity of the wind determined in the second step and from the magnetic configuration obtained in the first step, the current is derived. This process is then repeated until convergence is found. Figure 1.12 shows the converged solution of the Pneumann-Kopp model. The similarity to the solar corona is remarkable: the model gives rise to a region of closed magnetic field loops near the star at low latitudes and at high latitudes, open field lines that cannot restrain the expanding gas. This kind of structure is known as helmet streamer and will be discussed in more details in Chap. 4.

\subsection{Other Magnetized Wind Models}

In the previous sections, we presented three numerical models for the solar wind that will often be recalled along this thesis. Despite being simple models, they capture the pertinent physics of the solar wind, such as, the acceleration of the wind, the magnetic field configuration, etc. They all are axisymmetric, isothermal, and in steady state. These models are often employed in the description of magnetized wind models for other stars.

In addition to these classical models, several analytical studies were made toward understanding an expanding magnetized corona. For instance, Low and Tsinganos (1986) found a class of radial analytic solutions for magnetized steady-state winds by removing the latitude dependence of the problem. By varying the global magnetic field geometry 


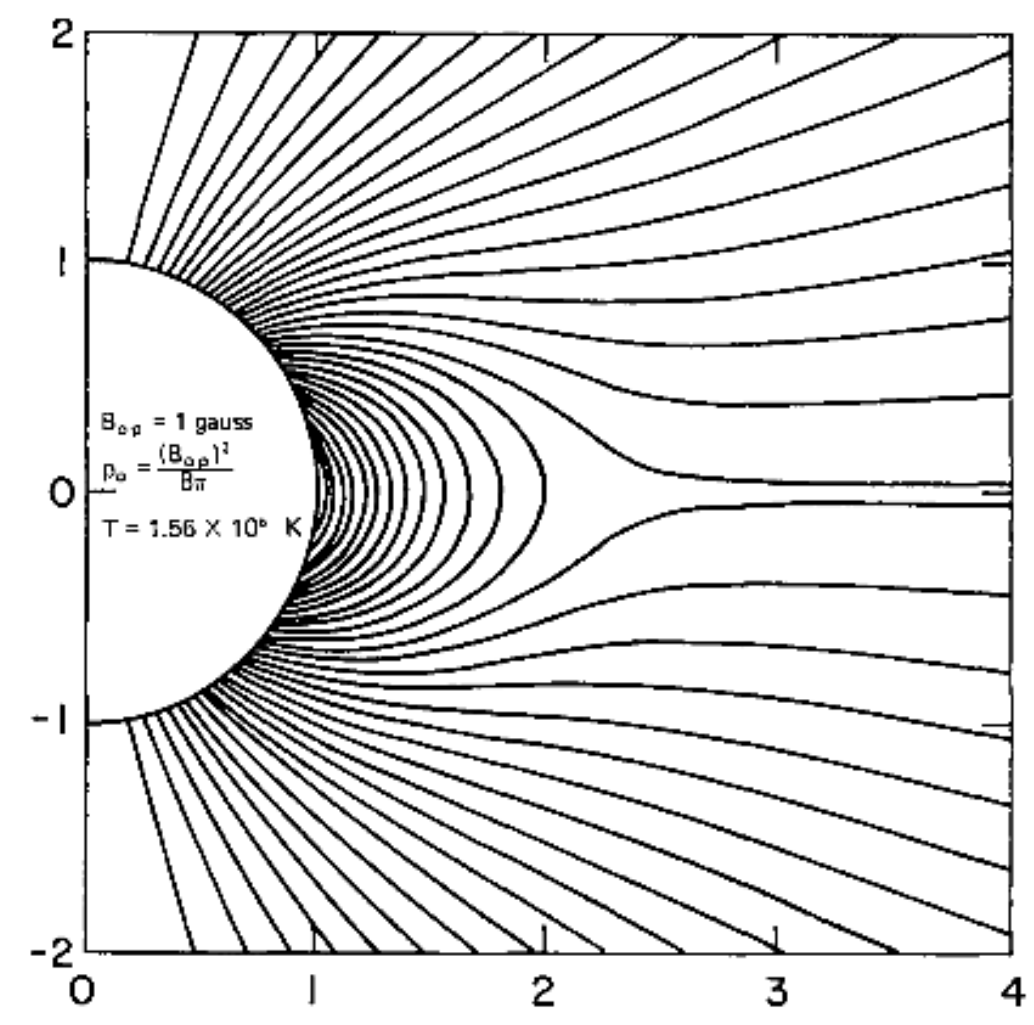

Figure 1.12: Magnetic field configuration self-consistently derived by an iterative method. Picture credit: Pneuman and Kopp (1971).

and other parameters of their model, such as the velocity at the base of the wind, they analyzed the behavior of flows along open magnetic field lines. Tsinganos and Trussoni (1991) relaxed the assumption of a polytropic equation of state, but also neglected the meridional (i.e., latitudinal) component of the flow. Sauty and Tsinganos (1994) found a new class of analytical solutions calculating the exact shape of a field line along the flow rather than assuming a global fixed magnetic field geometry. They showed that for a rotating young stellar object the shape of the field lines is an important parameter for the formation of a collimated jet/non-collimated wind. In a more recent work, Lima et al. (2001) constructed exact solutions for a rotating, magnetized wind. They verified that the wind is highly non-spherically symmetric. However, as they pointed out, their model has limitations, as, for instance, meridional flows and the meridional component of the magnetic field were neglected.

Meanwhile, numerical studies were carried out with increasing level of sophistication. Washimi and Shibata (1993) studied the influence of the stellar rotation on the wind 
structure and acceleration performing two-dimensional (2D) simulations. Solar wind parameters were used, except for the uniform angular velocity at the surface of the star that was varied as to analyze the effects of the centrifugal force. They showed that if the angular velocity is increased more than ten times the solar value, the centrifugal force becomes comparable to the thermal force, influencing the meridional structure of the wind. Keppens and Goedbloed (1999) presented detailed 1D and 2D ideal MHD numerical simulations of a polytropic, axisymmetric wind. In a posterior work, Keppens and Goedbloed (2000) modeled stellar axisymmetric rotating outflows by solving the ideal MHD equations, investigating the effects of open and closed magnetic field lines in the wind. By varying the extension of the closed field line region, as well as the intensity of the magnetic field, they showed that the global wind structure is modified. ud-Doula (2003), working towards the modeling of hot-star winds, presented a simulation of the solar wind using time-dependent, axisymmetric, MHD simulation. He showed that the MHD modeling was consistent with the work of Pneuman and Kopp (1971). Modeling the propagation of a coronal mass ejection from the inner solar corona to 1 AU, Groth et al. (2000) numerically reproduced the steady-state bimodal nature of the solar wind with a prescribed ad-hoc heating mechanism. Working on the modeling of the solar wind, Roussev et al. (2003) simulated the 3D structure of the solar wind under steadystate conditions, using solar magnetogram data as input parameters for the initiation of the wind, and considering a variable heating mechanism. Holzwarth (2005) analyzed the impact of a purely radial field on the wind. The stellar field, although radial, was taken to have a non-uniform distribution in the stellar surface, as to account for the non-uniform surface magnetic flux distribution observed in active stars. Cohen et al. (2007) extended Roussev et al.'s (2003) work considering, as a heating mechanism, a radial dependence of the ratio of specific heats, $\gamma=\gamma(r)$, as to reproduce the observed bimodality of the velocity of the solar wind. Matt and Pudritz (2005; 2008a;b) aiming at explaining the low rotation rates observed in CTTSs, studied the role of an accretion-powered stellar wind in the spinning down of a typical CTTS.

\subsection{Motivation}

Magnetic fields are often invoked to explain several scenarios along the life of a star, such as mass and angular momentum losses, collimation of jets, accretion through magnetic 
funnels, etc. In particular, the stellar magnetosphere plays an important role in the determination of the structure of a magnetized stellar wind, where either the intensity as well as the topology of the magnetic field are key ingredients. Therefore, a realistic determination of the coronal magnetic structure is crucial. Many difficulties in determining a realistic magnetic field structure are often encountered. For example, the origin of stellar magnetic field is still a matter of debate. Is the magnetic field fossil? Or is it created along the evolution of a star by internal dynamos? In stars with convective outer layers, the creation and amplification of magnetic fields are believed to arise from a combination of differential rotation and convection (Parker 1955, Babcock 1961). Magnetic field creation in fully convective stars, such as very low-mass stars, is still an open issue. These stars lack the interface layer where the dynamo is believed to operate. Nevertheless, they present large-scale, strong poloidal fields (Donati et al. 2006). Another difficulty in obtaining a realistic coronal magnetic field structure is that the stellar magnetic field can not be regarded as being in a vacuum, instead it is immersed in the stellar wind (Mestel 1968): on one hand, the outflowing wind deforms the magnetic field; on the other hand, the magnetic field redirects the stellar wind. The interplay between magnetic forces and the wind forces is what is going to determine the magnetic and wind structures (Pneuman and Kopp 1971).

In this thesis, we focus on the later issue and investigate both the magnetic and wind structures of magnetized stellar winds of MS solar-like stars and WTTSs. Despite all the notable evolution of both analytical and numerical studies performed in the last decades, we are far from a satisfactory three-dimensional (3D) magnetohydrodynamics (MHD) description of a magnetized wind. Several approximations are usually made in order to make the system analytically and numerically tractable (e.g., neglecting meridional flows, assuming a polytropic equation of state, assuming a fixed magnetic field topology).

To investigate magnetized coronal winds, we developed a novel 3D model of magnetized stellar winds, where we solve the fully 3D MHD equations with the temporal evolution using numerical simulations. Therefore, the topology of the field is not restricted in our model and the steady state arises from the dynamical interplay of the outflow and the field. Also, meridional flows arise naturally in the system. Our simulations were performed using the BATS-R-US code, developed at the University of Michigan and extensively used to simulate the Earth's magnetosphere, the heliosphere, and the magnetosphere of most of the planets and various comets of the Solar System. Our model 
is based on the work from Opher et al. (2004) for the outer heliosphere. BATS-R-US was extended to study magnetized coronal winds: a grid suitable for the problem was designed using the adaptive mesh refinement, and initial and boundary conditions for the simulations were carefully specified.

This thesis is organized in the following chapters. Since we are describing the stellar outflows by means of the MHD model, in Chap. 2 we derive the MHD equations that will be solved in the numerical code. Chapter 3 is dedicated to the numerical model that we use in the simulations. In Chap. 4, we present the work on magnetized wind of MS solar-like stars, analyzing cases with different plasma- $\beta$ parameters (the ratio of thermal to magnetic energy densities). This is the first study to perform such analysis solving the fully ideal 3D MHD equations. Chapter 5 presents a new approach for the inclusion of energy in stellar winds, by mimicking what is observed in the Sun. In Chap. 6, we present simulations of winds of WTTSs focusing mainly on the magnetic configuration of such winds. We explore several stellar and wind parameters constrained by observations, to investigate how the configuration of such winds is modified. We also investigate giant planet migrations due to an azimuthal drag force the wind exerts on the planet. In Chap. 7, we present preliminary results of a tilted stellar magnetosphere with respect to the rotational axis of the star. Last, we present in Chap. 8, a summary of this thesis, together with perspectives.

The refereed publications related to this thesis are enclosed in Appendix A. Chapters 4 and 6 present material that are published in peer-reviewed journals, while Chaps. 5 and 7 have yet unpublished work. Other topics of research in which I have been involved during my doctoral program are also presented in Appendix A, together with a reprint of such papers. 


\title{
Chapter 2
}

\section{Magnetohydrodynamics}

\begin{abstract}
Olasm
asma is the most common state of matter in the visible Universe and is usually described as a medium with positively and negatively charged particles that remains electrically neutral as a whole and exhibits collective behavior. The stars are made of plasma, as well as the medium between the stars, leading astronomers to believe that $90 \%$ of the visible Universe is made of plasma. The dynamics of a plasma may be described by different models. We focus here on the magnetohydrodynamics (MHD) model, which treats the plasma as a macroscopic single fluid. In this chapter, we present the general characteristics of the plasma and derive the equations of the MHD, which describe the temporal and spatial behavior of the following variables: the mass density $\rho$, pressure $p$ (or temperature $T$ ), velocity $\mathbf{u}$, and magnetic field $\mathbf{B}$.
\end{abstract}

\subsection{General Characteristics of the Plasma}

The properties of a plasma are determined by the interactions between its particles. The collective behavior is what differentiates the plasma from an ordinary fluid. Because the Coulomb force between electrically charged particles represents a long-range interaction, any particle of the plasma is able to interact simultaneously with a large number of nearby charged particles.

If there are no external forces, a small portion of a plasma (with a large number of particles) must be macroscopically neutral, i.e., there should not be any net resultant charge. In other words, each charged particle has an electric field associated that can be 
probed by other particles. However, the sum of the electric fields of each particle of the plasma cancel each other.

If a departure of macroscopic neutrality occurs, the particles of the plasma move as to restore the original charge neutrality. These collective motions are characterized by an oscillation, with a characteristic (angular) plasma frequency given by

$$
\omega_{p \alpha}=\left(\frac{4 \pi n_{\alpha} q_{\alpha}^{2}}{m_{\alpha}}\right)^{1 / 2}
$$

where $q_{\alpha}, m_{\alpha}$, and $n_{\alpha}$ are the charge, mass, and number density of electrons $(\alpha=e)$ and ions $(\alpha=i)$. Because the ions are more massive than the electrons, the oscillations of the electrons about the ions occur in a shorter timescale (i.e., electrons are faster than the ions).

The length-scale that the electric field of an individual particle influences the nearby particles is measured by the Debye length

$$
\lambda_{D}=\left(\frac{k_{B} T}{4 \pi n_{e} e^{2}}\right)^{1 / 2},
$$

where $e$ is the electron charge, $k_{B}$ the Boltzmann constant, and $T$ is the temperature. This has the consequence that each particle of the plasma is able to collectively interact with particles that are inside a volume with radius $\lambda_{D}$, known as Debye sphere.

One condition for the medium to be considered a plasma is that the characteristic dimension $D$ of the plasma is large compared to $\lambda_{D}$

$$
\lambda_{D} \ll D
$$

Another condition is that the average distance between the electrons $d \sim n_{e}^{-1 / 3}$ is small compared to $\lambda_{D}$, so that inside a Debye sphere there is a large number of particles enabling statistical considerations and electrical screening

$$
d \ll \lambda_{D}
$$

One last condition is that the collisions between electrons and neutral particles defined by a timescale $\tau_{e n}$ occur on a much larger timescale than the oscillatory motion of the plasma $\tau_{p \alpha} \sim 1 / \omega_{p \alpha}$

$$
\tau_{p \alpha} \ll \tau_{e n}
$$


If such condition is not valid, the electrons are not independent of the neutral particles and the fluid can be treated as an ordinary gas.

So far, we have not made any consideration about magnetic fields. However, magnetic fields play important roles in Astrophysical Plasmas. In a magnetized plasma, another important scale is set by the gyrofrequency or cyclotron frequency: a charged particle circulates around the magnetic field lines with an angular frequency given by

$$
\Omega_{c \alpha}=\frac{\left|q_{\alpha}\right| B}{m_{\alpha} c},
$$

where $B$ is the magnitude of the magnetic field and $c$ is the speed of light. If we shall describe the plasma with a macroscopic model, the macroscopic timescale of the dynamics of a plasma $\tau$ should be longer than $\tau_{c \alpha} \sim 1 / \Omega_{c \alpha}$,

$$
\tau \gg \tau_{c \alpha},
$$

and the characteristic length $D$ of the plasma much larger than the cyclotron radius or Larmor radius

$$
D \gg R_{c \alpha}
$$

with

$$
R_{c \alpha}=\frac{m_{\alpha} u_{\perp} c}{\left|q_{\alpha}\right| B}
$$

where $u_{\perp}$ is the velocity of the particle that is perpendicular to the magnetic field line.

\subsection{Derivation of the MHD Equations}

The magnetohydrodynamics equations are used to compute macroscopic observables of a conducting fluid as a whole, opposed to the microscopic physical details that are computed by the kinetic plasma theory. In the derivation of the MHD equations from the kinetic theory, the dynamics of the fluid is calculated performing integrations of the Boltzmann equation over different moments, thus averaging out any microscopic fluctuation. We omit such derivation here and refer the reader to textbooks such as Gombosi (1998), Bittencourt (2004), Goedbloed and Poedts (2004). Here, instead, we derive the MHD equations in a more simplistic way: by merging the fluid mechanics equations (gas dynamics) and the Maxwell's equations of the electromagnetism. 


\subsubsection{Preliminaries}

First, consider the Maxwell's equations in vacuum (Gaussian units):

$$
\begin{aligned}
& \nabla \cdot \mathbf{E}=4 \pi \rho_{e}, \\
& \nabla \times \mathbf{E}=-\frac{1}{c} \frac{\partial \mathbf{B}}{\partial t}, \\
& \nabla \cdot \mathbf{B}=0, \\
& \nabla \times \mathbf{B}=\frac{4 \pi}{c} \mathbf{J}+\frac{1}{c} \frac{\partial \mathbf{E}}{\partial t},
\end{aligned}
$$

where $\mathbf{E}$ and $\mathbf{B}$ are the electric and magnetic field vectors, $\rho_{e}$ the charge density, and $\mathbf{J}$ is the current density.

Let us now present the gas dynamics equations that we shall use. The equation that describes the conservation of mass is the continuity equation

$$
\frac{\partial \rho}{\partial t}+\nabla \cdot(\rho \mathbf{u})=0
$$

Another way to write the previous equation is

$$
\frac{\mathrm{D} \rho}{\mathrm{D} t}=-\rho \nabla \cdot \mathbf{u}
$$

where the differential operator $\mathrm{D} / \mathrm{D}$ t is the total derivative, also known as Lagrangian derivative, and is a time derivative that follows the motion of a fluid element. In Euler's notation, we have

$$
\frac{\mathrm{D}}{\mathrm{D} t}=\frac{\partial}{\partial t}+(\mathbf{u} \cdot \nabla)
$$

The total derivative is a scalar operator that includes both space and time partial derivatives.

If a fluid element is subject to pressure gradients and gravitational forces, the momentum equation (equation of motion) is

$$
\rho \frac{\mathrm{D} \mathbf{u}}{\mathrm{D} t}=-\nabla p+\rho \mathbf{g}
$$

If there are additional forces, such as viscous forces, electromagnetic force, etc., they are added in the right hand side (RHS) of Eq. (2.17). In Eulerian notation, Eq. (2.17) is written as

$$
\rho\left(\frac{\partial \mathbf{u}}{\partial t}+(\mathbf{u} \cdot \nabla) \mathbf{u}\right)=-\nabla p+\rho \mathbf{g}
$$


Even if the fluid is steady, i.e., $\partial \mathbf{u} / \partial t=0$, there may be an acceleration of the fluid, as the total derivative may not be null $([\mathbf{u} \cdot \nabla] \mathbf{u} \neq 0)$. The term $(\mathbf{u} \cdot \nabla) \mathbf{u}$ represents the change of $\mathbf{u}$ in the direction of $\mathbf{u}$ : acceleration occurs when streamlines of velocity (i.e., the trajectories of the fluid elements) change direction.

Another equation from gas dynamics we will be using later on is the energy equation. From the first law of thermodynamics, the internal energy $U$ of a fluid is related to the work $W$ and to the energy gained $Q$ as

$$
\frac{\mathrm{D} U}{\mathrm{D} t}=\frac{\mathrm{D} W}{\mathrm{D} t}+\frac{\mathrm{D} Q}{\mathrm{D} t}
$$

where viscous processes have been neglected and $U, W$, and $Q$ are measures of energy per unit mass. The work is written as $W=-p \mathrm{~d} V=-p \mathrm{~d}(1 / \rho)$, hence

$$
\frac{\mathrm{D} W}{\mathrm{D} t}=\frac{p}{\rho^{2}} \frac{\mathrm{D} \rho}{\mathrm{D} t}
$$

Writing

$$
\frac{\mathrm{D} Q}{\mathrm{D} t}=-\dot{Q}
$$

where $\dot{Q}>0$ for cooling and $\dot{Q}<0$ for heating, the first law of thermodynamics is rewritten as

$$
\frac{\mathrm{D} U}{\mathrm{D} t}=\frac{p}{\rho^{2}} \frac{\mathrm{D} \rho}{\mathrm{D} t}-\dot{Q}
$$

The total energy $\epsilon$ per unit volume is defined as the sum of the kinetic energy, the internal energy, and the potential energy

$$
\epsilon=\frac{\rho u^{2}}{2}+\rho U+\rho \psi=\rho\left(\frac{u^{2}}{2}+U+\psi\right)
$$

where $\psi$ is the gravitational potential such that $\mathbf{g}=-\nabla \psi$. Differentiating the previous equation, we have

$$
\frac{\mathrm{D} \epsilon}{\mathrm{D} t}=\frac{\epsilon}{\rho} \frac{\mathrm{D} \rho}{\mathrm{D} t}+\rho\left(\mathbf{u} \cdot \frac{\mathrm{D} \mathbf{u}}{\mathrm{D} t}+\frac{\mathrm{D} U}{\mathrm{D} t}+\frac{\mathrm{D} \psi}{\mathrm{D} t}\right)
$$

Substituting Eqs. (2.15), (2.16), (2.17), and (2.22) into Eq. (2.24), and setting $\partial \psi / \partial t=0$, we have, after rearranging terms, the energy equation from gas dynamics

$$
\frac{\partial \epsilon}{\partial t}+\nabla \cdot[(\epsilon+p) \mathbf{u}]=-\rho \dot{Q} .
$$




\subsubsection{Some Useful Plasma Relations}

If we consider a plasma of electrons (with charge $q_{e}=-e$ ) and ions (with charge $q_{i}=$ $+Z e)$, then

$$
\rho_{e}=\sum_{\alpha=i, e} n_{\alpha} q_{\alpha}=n_{i} Z e-n_{e} e
$$

and

$$
\mathbf{J}=\sum_{\alpha=i, e} n_{\alpha} q_{\alpha} \mathbf{v}_{\alpha}=n_{i} Z e \mathbf{v}_{\mathbf{i}}-n_{e} e \mathbf{v}_{\mathbf{e}}=\rho_{e} \mathbf{v}_{\mathbf{i}}-n_{e} e \mathbf{V}
$$

where $\mathbf{V}=\mathbf{v}_{\mathbf{e}}-\mathbf{v}_{\mathbf{i}}$ is the drift velocity of electrons relative to ions. If the plasma is electrically neutral, $n_{i} Z e \simeq n_{e} e$, and from Eq. (2.26), $\rho_{e} \simeq 0$. Using this result in Eq. (2.27), we have

$$
\mathbf{J} \simeq-n_{e} e \mathbf{V}
$$

As $m_{i} \gg m_{e}$, the plasma density is given by

$$
\rho=\sum_{\alpha=i, e} n_{\alpha} m_{\alpha}=n_{i} m_{i}+n_{e} m_{e} \simeq n_{i} m_{i}
$$

Using Eq. (2.29) and $m_{i} \gg m_{e}$, we write the bulk velocity ${ }^{1} \mathbf{u}$ of the plasma as

$$
\mathbf{u}=\frac{n_{i} m_{i} \mathbf{v}_{\mathbf{i}}+n_{e} m_{e} \mathbf{v}_{\mathbf{e}}}{n_{i} m_{i}+n_{e} m_{e}} \simeq \frac{n_{e} m_{e}}{n_{i} m_{i}} \mathbf{v}_{\mathbf{e}}+\mathbf{v}_{\mathbf{i}} \simeq \mathbf{v}_{\mathbf{i}}
$$

The last equation shows that the bulk velocity of the plasma is essentially the velocity of the ions.

\subsubsection{The Generalized Ohm's Law}

Our goal now is to derive a relation between the current density and the electric field, which is called generalized Ohm's law. To derive the generalized Ohm's law, we will use Eqs. (2.28) and (2.30). We start with the equation of motion for the electrons

$$
n_{e} m_{e} \frac{\mathrm{D} \mathbf{v}_{\mathbf{e}}}{\mathrm{D} t}=-\nabla p_{e}+n_{e} m_{e} \mathbf{g}-n_{e} e\left(\mathbf{E}+\frac{\mathbf{v}_{\mathbf{e}} \times \mathbf{B}}{c}\right)+n_{e} \mathbf{F}_{\mathbf{e i}}
$$

\footnotetext{
${ }^{1}$ This is the center-of-mass velocity.
} 
where in the left hand side of Eq. (2.31) we find the acceleration term of the electrons; in the RHS, the terms are, respectively, the gradient of the thermal pressure $p_{e}$ of the electrons, the gravity force acting on the electrons, the electromagnetic force, and $\mathbf{F}_{\mathbf{e i}}$ is the frictional force per electron due to electron-ion collisions. If we suppose that the collisions take place at intervals $\tau_{e i}$, we can write the collisional force as

$$
\mathbf{F}_{\mathbf{e i}}=-\frac{m_{e} \mathbf{V}}{\tau_{e i}}
$$

By ignoring the inertia of electrons (i.e., $n_{e} m_{e} \mathrm{D} \mathbf{v}_{\mathbf{e}} / \mathrm{D} t \simeq 0$ ), we are assuming that the timescales of the plasma are large when compared to both the cyclotron period $\left(\tau \gg \tau_{c \alpha}\right)$ and to the plasma oscillation period $\left(\tau \gg \tau_{p \alpha}\right)$. Hence, the terms in the RHS of Eq. (2.31) are always in equilibrium. Using $\mathbf{V}=\mathbf{v}_{\mathbf{e}}-\mathbf{v}_{\mathbf{i}}$ and Eq. (2.32), we have

$$
-\nabla p_{e}+n_{e} m_{e} \mathbf{g}-n_{e} e\left(\mathbf{E}+\frac{\mathbf{V} \times \mathbf{B}}{c}\right)-n_{e} e \frac{\mathbf{v}_{\mathbf{i}} \times \mathbf{B}}{c}-n_{e} \frac{m_{e} \mathbf{V}}{\tau_{e i}} \simeq 0 .
$$

We now use the results derived in Eq. (2.28) and (2.30), and the previous equation reads

$$
-\frac{\nabla p_{e}}{n_{e} e}+\frac{m_{e} \mathbf{g}}{e}-\mathbf{E}+\frac{\mathbf{J} \times \mathbf{B}}{c n_{e} e}-\frac{\mathbf{u} \times \mathbf{B}}{c}+\frac{m_{e} \mathbf{J}}{e^{2} n_{e} \tau_{e i}} \simeq 0 \text {. }
$$

As the gravitational force on the electrons is very small, we can neglect this term in the previous equation. Defining the electrical conductivity as

$$
\sigma=\frac{n_{e} e^{2} \tau_{e i}}{m_{e}}=\frac{\omega_{p e}^{2} \tau_{e i}}{4 \pi}
$$

we find the generalized Ohm's law

$$
\mathbf{J}=\sigma\left(\mathbf{E}+\frac{\mathbf{u} \times \mathbf{B}}{c}\right)-\frac{\sigma}{c n_{e} e}(\mathbf{J} \times \mathbf{B})+\frac{\sigma}{n_{e} e} \nabla p_{e} .
$$

The term $\sigma \nabla p_{e} /\left(n_{e} e\right)$ is the term that gives rise to the Biermann battery term in the induction equation (to be derived next) and it is generally neglected. The term $-\sigma(\mathbf{J} \times \mathbf{B}) /\left(c n_{e} e\right)$ describes the Hall effect. Let us see why it is also usually neglected. Using Eqs. (2.6) and (2.35), the ratio between the Hall term and the Ohm term is

$$
\frac{\left|\sigma(\mathbf{J} \times \mathbf{B}) /\left(c n_{e} e\right)\right|}{|\mathbf{J}|} \sim \frac{\sigma B}{c n_{e} e} \sim \frac{\tau_{e i}}{\tau_{c e}} .
$$

If the interval between electron-ion collisions $\tau_{e i}$ is much larger than the time $\tau_{c e}$ required for an electron to complete a gyration around the magnetic field line, then $\tau_{e i} / \tau_{c e} \gg 1$ and the Hall term can be neglected.

With these assumptions, the Ohm's law typically used in the MHD theory is then

$$
\mathbf{J}=\sigma\left(\mathbf{E}+\frac{\mathbf{u} \times \mathbf{B}}{c}\right) \text {. }
$$




\subsubsection{The Induction Equation}

In the original Ampére's law, there is no displacement current $\left(c^{-1} \partial \mathbf{E} / \partial t\right)$ in Eq. (2.13). In MHD, the term $\partial \mathbf{E} / \partial t$ is also usually neglected. To show that, we perform an order of magnitude calculation. From Eq. (2.11), we have

$$
\frac{c|\nabla \times \mathbf{E}|}{|\partial \mathbf{B} / \partial t|} \sim \frac{E}{B} \frac{c \tau}{D} \sim \frac{E}{B} \frac{c}{u} \sim 1
$$

I.e., $E / B \sim u / c$, where we assumed that the ratio between the characteristic length-scale $D$ over the timescale $\tau$ of the plasma is $u$. Substituting this result in the ratio between the terms of Eq. (2.13) containing the electric and magnetic field vectors, we have

$$
\frac{|\partial \mathbf{E} / \partial t|}{c|\nabla \times \mathbf{B}|} \sim \frac{E / \tau}{c B / D} \sim \frac{u}{c^{2} \tau / D} \sim \frac{u^{2}}{c^{2}}
$$

For a non-relativistic flow, it is reasonable to assume that $u^{2} / c^{2} \ll 1$, thus showing that we can safely neglect the displacement current in Eq. (2.13). Hence, we recover the original Ampére's law

$$
\mathbf{J}=\frac{c}{4 \pi}(\nabla \times \mathbf{B})
$$

Substituting Eqs. (2.38) and (2.41) in Eq. (2.11), we find

$$
\frac{\partial \mathbf{B}}{\partial t}=-\nabla \times\left(c \frac{\mathbf{J}}{\sigma}-\mathbf{u} \times \mathbf{B}\right)=-\nabla \times\left[\frac{c^{2}}{4 \pi \sigma}(\nabla \times \mathbf{B})-\mathbf{u} \times \mathbf{B}\right],
$$

and the induction equation of the magnetic field is

$$
\frac{\partial \mathbf{B}}{\partial t}=\nabla \times(\mathbf{u} \times \mathbf{B})+\eta \nabla^{2} \mathbf{B}
$$

where $\eta=c^{2} /(4 \pi \sigma)$ is the diffusion coefficient that in the previous deduction was assumed to be constant in space.

The second term in the RHS of Eq. (2.43) represents the diffusion of the magnetic field vector. The ratio between the first and second terms in Eq. (2.43) is known as the magnetic Reynolds number:

$$
R_{m}=\frac{|\nabla \times(\mathbf{u} \times \mathbf{B})|}{\left|\eta \nabla^{2} \mathbf{B}\right|} \sim \frac{D u}{\eta} .
$$

The magnetic Reynolds number $R_{m}$ measures the relative importance between the convective and diffusive terms of Eq. (2.43). In most plasmas of interest, $\eta$ is very small 
(which means that the electrical conductivity $\sigma$ is very large), implying in $R_{m} \gg 1$. When $R_{m} \gg 1$, diffusion can be neglected and Eq. (2.43) describes the evolution of the magnetic field in the ideal MHD approach

$$
\frac{\partial \mathbf{B}}{\partial t}=\nabla \times(\mathbf{u} \times \mathbf{B}) .
$$

\subsubsection{The Equation of Motion for the Plasma}

Similar to what we have done for the electrons [Eq. (2.31)], we write the equation of motion for the ions

$$
n_{i} m_{i} \frac{\mathrm{D} \mathbf{v}_{\mathbf{i}}}{\mathrm{D} t}=-\nabla p_{i}+n_{i} m_{i} \mathbf{g}+n_{i} Z e\left(\mathbf{E}+\frac{\mathbf{v}_{\mathbf{i}} \times \mathbf{B}}{c}\right)+n_{i} \mathbf{F}_{\mathbf{i e}}
$$

The frictional force of the ion-electron collision obeys the relation $n_{i} \mathbf{F}_{\mathbf{i e}}=-n_{e} \mathbf{F}_{\mathbf{e i}}$ and using Eq. (2.31), it can be written as

$$
n_{i} \mathbf{F}_{\mathbf{i}, \mathbf{e}}=-n_{e} \mathbf{F}_{\mathbf{e i}}=-\nabla p_{e}+n_{e} m_{e} \mathbf{g}-n_{e} e\left(\mathbf{E}+\frac{\mathbf{v}_{\mathbf{e}} \times \mathbf{B}}{c}\right)
$$

where we have neglected the acceleration term of the electrons. Substituting Eq. (2.47) in Eq. (2.46), and rearranging terms, we have

$$
n_{i} m_{i} \frac{\mathrm{D} \mathbf{v}_{\mathbf{i}}}{\mathrm{D} t}=-\nabla\left(p_{i}+p_{e}\right)+\left(n_{i} m_{i}+n_{e} m_{e}\right) \mathbf{g}+\left(n_{i} Z e-n_{e} e\right) \mathbf{E}+\left(n_{i} Z e \mathbf{v}_{\mathbf{i}}-n_{e} e \mathbf{v}_{\mathbf{e}}\right) \times \frac{\mathbf{B}}{c}
$$

We can rearrange last equation by remembering that: (1) The sum of the partial pressures is the total pressure $p=p_{i}+p_{e}$. (2) Using Eq. (2.29), we have that $\rho \simeq n_{i} m_{i}$. (3) If the plasma is electrically neutral, $n_{i} Z e \simeq n_{e} e$ [Eq. (2.26)]. Hence, the term containing the electric field vanishes. (4) $\mathbf{J}=n_{i} Z e \mathbf{v}_{\mathbf{i}}-n_{e} e \mathbf{v}_{\mathbf{e}}$ from Eq. (2.27). (5) From Eq. (2.30), $\mathbf{v}_{\mathbf{i}} \simeq \mathbf{u}$. Hence, we have

$$
\rho \frac{\mathrm{D} \mathbf{u}}{\mathrm{D} t}=-\nabla p+\rho \mathbf{g}+\frac{\mathbf{J} \times \mathbf{B}}{c}
$$

or in Eulerian notation

$$
\rho\left(\frac{\partial \mathbf{u}}{\partial t}+(\mathbf{u} \cdot \nabla) \mathbf{u}\right)=-\nabla p+\rho \mathbf{g}+\frac{\mathbf{J} \times \mathbf{B}}{c} .
$$




\section{The Lorentz Force}

We shall concentrate next in the Lorentz force. Using Eq. (2.41)

$$
\mathbf{F}_{\mathbf{B}}=\frac{\mathbf{J} \times \mathbf{B}}{c}=\frac{(\nabla \times \mathbf{B}) \times \mathbf{B}}{4 \pi}=\frac{(\mathbf{B} \cdot \nabla) \mathbf{B}}{4 \pi}-\nabla\left(\frac{B^{2}}{8 \pi}\right)
$$

The second term on the RHS of Eq. (2.51) is the gradient of the magnetic pressure $P_{B}=B^{2} /(8 \pi)$. The first term on the RHS of Eq. (2.51) only contributes to the Lorentz force when the field varies along the direction of $\mathbf{B}$. It is the tension force of the line. If we write $\mathbf{B}=B \hat{\mathbf{s}}$, where $\hat{\mathbf{s}}$ is the unit vector along $\mathbf{B}$ as shown in Fig. 2.1, then the tension is

$$
\mathcal{T}_{\mathbf{B}}=\frac{(\mathbf{B} \cdot \nabla) \mathbf{B}}{4 \pi}=\frac{B}{4 \pi} \frac{\mathrm{d}(B \hat{\mathbf{s}})}{\mathrm{d} s}=\frac{\mathrm{d}}{\mathrm{d} s}\left(\frac{B^{2}}{8 \pi}\right) \hat{\mathbf{s}}+\frac{B^{2}}{4 \pi} \frac{\mathrm{d} \hat{\mathbf{s}}}{\mathrm{d} s}=\nabla_{\|}\left(\frac{B^{2}}{8 \pi}\right)+\frac{B^{2}}{4 \pi} \frac{\hat{\mathbf{n}}}{\varpi_{c}},
$$

where $\hat{\mathbf{n}}$ is the unit vector normal to the field line (i.e., $\hat{\mathbf{n}} \perp \hat{\mathbf{s}}$ ), and $\varpi_{c}$ is the local radius of curvature of the line. The tension force is larger the more curved the line is (implying in low $\varpi_{c}$ ). Substituting Eq. (2.52) into Eq. (2.51)

$$
\mathbf{F}_{\mathbf{B}}=\nabla_{\|}\left(\frac{B^{2}}{8 \pi}\right)+\frac{B^{2}}{4 \pi} \frac{\hat{\mathbf{n}}}{\varpi_{c}}-\nabla\left(\frac{B^{2}}{8 \pi}\right)=\frac{B^{2}}{4 \pi} \frac{\hat{\mathbf{n}}}{\varpi_{c}}-\nabla_{\perp}\left(\frac{B^{2}}{8 \pi}\right) .
$$

We see from Eq. (2.53) that the component along the field line cancels and that the Lorentz force is perpendicular to $\mathbf{B}$. Here we showed that the Lorentz force $\mathbf{F}_{\mathbf{B}}$ acting on the flow is given by the difference between the magnetic tension, $\mathcal{T}_{\mathbf{B}}$, and the gradient of the magnetic pressure $P_{B}$. The tension acts as to resist the curvature of the lines, while $\nabla P_{B}$ acts in a similar fashion as the thermal pressure gradient in Eq. (2.49).

\subsubsection{The Energy Equation}

To derive the energy equation, we will start from the Poynting theorem. Taking the dot product of Eq. (2.11) with $\mathbf{B}$ and adding the resultant equation to the dot product of Eq. (2.13) with $-\mathbf{E}$ yields

$$
\frac{\partial}{\partial t}\left(\frac{B^{2}}{8 \pi}\right)+\nabla \cdot\left[\frac{c}{4 \pi}(\mathbf{E} \times \mathbf{B})\right]=-\mathbf{J} \cdot \mathbf{E}
$$




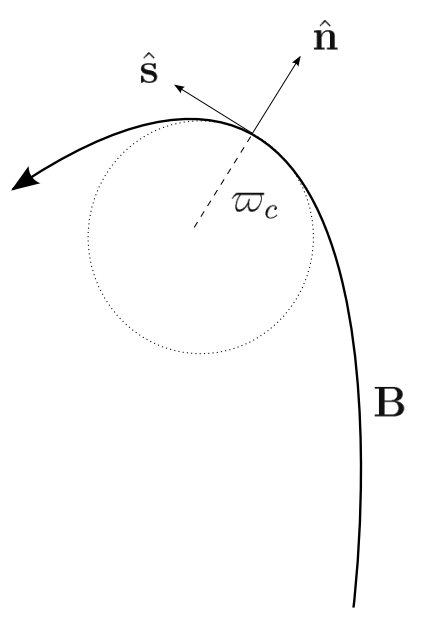

Figure 2.1: Orientation of the unit vectors $\hat{\mathbf{s}}$ and $\hat{\mathbf{n}}$, along and perpendicular to the field line, respectively; $\varpi_{c}$ is the local radius of curvature of the line.

where we already have neglected the displacement current. Let us simplify Eq. (2.54) by solving for its terms separately. The term with the divergent is

$$
\begin{aligned}
\nabla \cdot\left[\frac{c}{4 \pi}(\mathbf{E} \times \mathbf{B})\right] & =\nabla \cdot\left[\frac{c}{4 \pi \sigma}(\mathbf{J} \times \mathbf{B})-\frac{(\mathbf{u} \times \mathbf{B})}{4 \pi} \times \mathbf{B}\right] \\
& =\nabla \cdot\left[\frac{c}{4 \pi \sigma}(\mathbf{J} \times \mathbf{B})+\frac{B^{2}}{4 \pi} \mathbf{u}-\frac{(\mathbf{u} \cdot \mathbf{B})}{4 \pi} \mathbf{B}\right]
\end{aligned}
$$

where we used [Eq. (2.38)]

$$
\mathbf{E}=\frac{\mathbf{J}}{\sigma}-\frac{\mathbf{u} \times \mathbf{B}}{c}
$$

in the first equality. The RHS of Eq. (2.54) is

$$
-\mathbf{J} \cdot \mathbf{E}=-\frac{J^{2}}{\sigma}-\frac{(\mathbf{J} \times \mathbf{B})}{c} \cdot \mathbf{u}
$$

Substituting Eqs. (2.56) and (2.58) in (2.54), and setting $(\mathbf{J} \times \mathbf{B}) \cdot \mathbf{u} / c=0$, we find

$$
\frac{\partial}{\partial t}\left(\frac{B^{2}}{8 \pi}\right)+\nabla \cdot\left[\frac{c}{4 \pi \sigma}(\mathbf{J} \times \mathbf{B})+\frac{B^{2}}{4 \pi} \mathbf{u}-\frac{(\mathbf{u} \cdot \mathbf{B})}{4 \pi} \mathbf{B}\right]=-\frac{J^{2}}{\sigma}
$$

We now use the energy equation for gas dynamics deduced before. Adding Eq. (2.25) and (2.59), we have

$$
\frac{\partial}{\partial t}\left(\epsilon+\frac{B^{2}}{8 \pi}\right)+\nabla \cdot\left[(\epsilon+p) \mathbf{u}+\frac{c}{4 \pi \sigma}(\mathbf{J} \times \mathbf{B})+\frac{B^{2}}{4 \pi} \mathbf{u}-\frac{(\mathbf{u} \cdot \mathbf{B})}{4 \pi} \mathbf{B}\right]=-\rho \dot{Q}-\frac{J^{2}}{\sigma} .
$$


The internal energy for an ideal gas is

$$
U=\frac{1}{(\gamma-1)} \frac{p}{\rho}
$$

where $\gamma$ is the ratio of the specific heats.

In ideal MHD, the conductive is very large, so $1 / \sigma \rightarrow 0$. Let us also consider that there is no extra term of heating and cooling in the system such that $\dot{Q}=0$ (the entropy of the system remains constant). Hence, the energy equation becomes

$$
\begin{aligned}
& \frac{\partial}{\partial t}\left[\rho\left(\frac{u^{2}}{2}+\frac{1}{\gamma-1} \frac{p}{\rho}+\psi\right)+\frac{B^{2}}{8 \pi}\right] \\
& +\nabla \cdot\left[\rho\left(\frac{u^{2}}{2}+\frac{\gamma}{\gamma-1} \frac{p}{\rho}+\psi\right) \mathbf{u}+\frac{B^{2}}{4 \pi} \mathbf{u}-\frac{(\mathbf{u} \cdot \mathbf{B})}{4 \pi} \mathbf{B}\right]=0
\end{aligned}
$$

where we have used Eqs. (2.23) and (2.61).

\section{$2.3 \quad$ Final Remarks}

In three dimensions, the MHD equations consist of eight equations:

a) one equation for the conservation of mass [Eq. (2.14)],

b) three equations for the conservation of momentum [Eq. (2.50)],

c) three equations for the magnetic field (induction) [Eq. (2.45)], and

d) one equation for the energy conservation [Eq. (2.62)].

These eight equations are then solved for eight variables, namely the density $\rho$, the velocity $\mathbf{u}=\left\{u_{x}, u_{y}, u_{z}\right\}$, the magnetic field $\mathbf{B}=\left\{B_{x}, B_{y}, B_{z}\right\}$, and the gas pressure $p$. These variables are known as primitive variables. 


\section{Chapter 3}

\section{Our 3D Numerical Model}

\footnotetext{
G

olving the MHD equations can be very intricate, since they are generally coupled to each other. Unless several approximations are made, numerical methods are needed to solve such equations. To perform the simulations that are presented in the next chapters, we use the numerical code BATS-R-US, which solves the full set of 3D MHD equations. In this chapter, we describe the main characteristics of such tool and the user routines implemented to study magnetized winds.
}

\subsection{BATS-R-US}

The Block Adaptive Tree Solar-wind Roe Upwind Scheme (BATS-R-US) was developed at the University of Michigan (Gombosi et al. 1994, Powell et al. 1999). It is a parallelized code, written completely in standard Fortran 90, and its structure is block-based. The blocks are distributed on different processors and must communicate with each other through message passing. Each block occupies the same amount of space in memory, but they may occupy different sized volumes in physical space. The adaptive mesh refinement (AMR) technique implemented in BATS-R-US allows to create and store complex grids. BATS-R-US solves the MHD equations in a finite-volume scheme and uses high-performance MHD algorithms (such as upwind methods, approximate Riemann solvers, and limited solution reconstruction). We refer the reader to, e.g., Powell (1994), Powell et al. (1999), Tóth (2000), De Zeeuw et al. (2000), Clauer et al. (2000), Gombosi et al. (2004), Tóth et al. (2005; 2006) for details of these numerical methods. The validity 
and accuracy of the scheme are discussed by Powell et al. (1999).

The code solves the coupled ideal MHD equations that were discussed in Chap. 2. The ideal MHD equations can be written in the conservative form, i.e., in the following format

$$
\frac{\partial}{\partial t}(\ldots)+\nabla \cdot(\ldots)=0
$$

If the equation does not follow the format given in Eq. (3.1) and is instead $\neq 0$, the term on the RHS of the equation is known as a source term.

The equations derived in Chap. 2 [Eqs. (2.14), (2.45), (2.50), and (2.62)] can be transformed to the conservative form [Eq. (3.1)] (Goedbloed and Poedts 2004). The equations solved by BATS-R-US are thus given by

$$
\begin{aligned}
& \frac{\partial \rho}{\partial t}+\nabla \cdot(\rho \mathbf{u})=0 \\
& \frac{\partial(\rho \mathbf{u})}{\partial t}+\nabla \cdot\left[\rho \mathbf{u} \mathbf{u}+\left(p+\frac{B^{2}}{8 \pi}\right) I-\frac{\mathbf{B} \mathbf{B}}{4 \pi}\right]=\rho \mathbf{g} \\
& \frac{\partial \mathbf{B}}{\partial t}+\nabla \cdot(\mathbf{u} \mathbf{B}-\mathbf{B} \mathbf{u})=0 \\
& \frac{\partial \varepsilon}{\partial t}+\nabla \cdot\left[\mathbf{u}\left(\varepsilon+p+\frac{B^{2}}{8 \pi}\right)-\frac{(\mathbf{u} \cdot \mathbf{B}) \mathbf{B}}{4 \pi}\right]=\rho \mathbf{g} \cdot \mathbf{u},
\end{aligned}
$$

where $I$ is the identity matrix and $\varepsilon$ is the total energy density (excluding gravitational potential energy)

$$
\varepsilon=\frac{\rho u^{2}}{2}+\frac{p}{\gamma-1}+\frac{B^{2}}{8 \pi}
$$

Here, we note that Eqs. (3.3) and (3.5) present source terms that are related to the potential gravitational field.

One of the great advantages of BATS-R-US is that it can be adaptively refined, increasing or decreasing the resolution of the simulation in specific portions of the computational domain. To do that, an AMR technique is used in the MHD algorithm of BATS-R-US. BATS-R-US uses a Cartesian computational domain that is block-based, where blocks can be created or destroyed as the computation proceeds. The creation of blocks is illustrated as follows. Each parent-block is divided in eight $(2 \times 2 \times 2)$, generating eight other blocks known as children. This procedure doubles the resolution of the simulation in the region where blocks are created. Every time a block is divided, one 


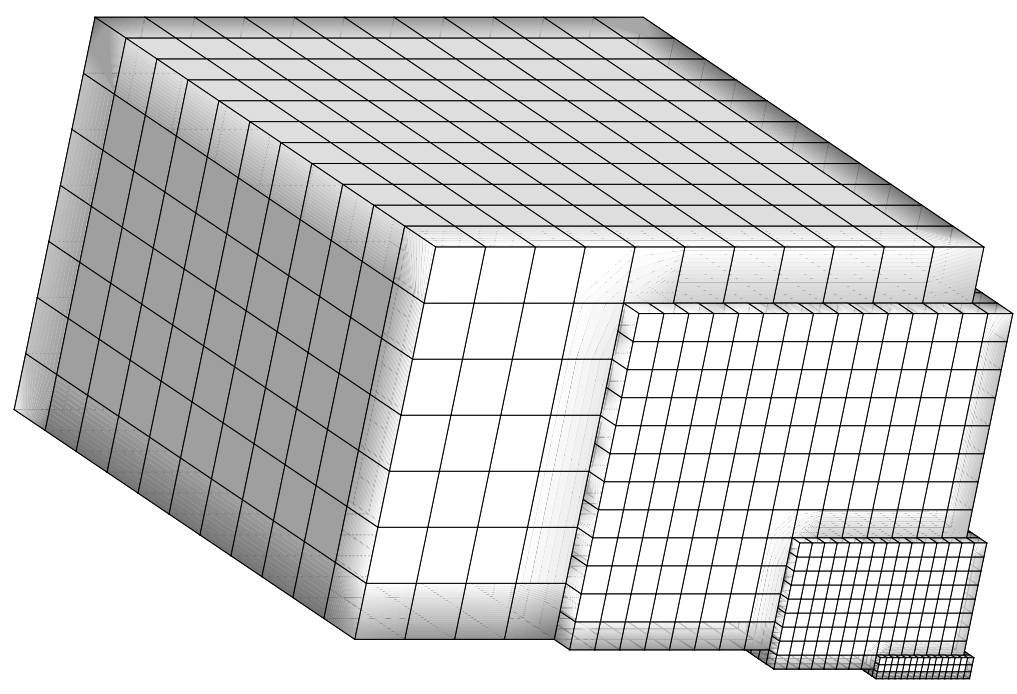

Figure 3.1: The block-adaptive grid structure. In the figure, four different levels of refinement are shown.

level of refinement is added. Figure 3.1 illustrates a grid structure, where four different levels of refinement are shown. Each block contains 64 cells $(4 \times 4 \times 4)$ and the equations are solved in each cell, as will be shown in $§ 3.1 .2$.

The user sets the case where the code should use more resolution: the case can be based on a dynamical characteristic of the simulation (e.g., the increase on the density in a shock region) or on geometrical characteristics (e.g., around a planet sitting on a specific position of the grid).

\subsubsection{Normalized Units}

The MHD equations can be made dimensionless by means of three quantities for the units of length, velocity, and density, which are the basic parameters

$$
\begin{aligned}
& r=r^{\prime} r_{0} \\
& \mathbf{u}=\mathbf{u}^{\prime} u_{0} \\
& \rho=\rho^{\prime} \rho_{0}
\end{aligned}
$$

where the variables with ' are dimensionless variables and $r_{0}, u_{0}$, and $\rho_{0}$ are characteristic constants of the problem. In our simulations, for instance, the characteristic length $r_{0}$ 
is set to be the radius of the star $r_{0}$, while the characteristic velocity $u_{0}$ is the sound velocity, and the characteristic density $\rho_{0}$ is the density at the base of the wind. The other normalization constants follow by exploiting plasma dynamics as presented next.

- Time: $t=t^{\prime} t_{0}$, where $t_{0}$ is derived by the characteristic time (the ratio between the characteristic distance and the characteristic velocity)

$$
t_{0}=\frac{r_{0}}{u_{0}}
$$

- Pressure: $p=p^{\prime} p_{0}$, where $p_{0}$ is derived by the ram pressure of the plasma

$$
p_{0}=\rho_{0} u_{0}^{2}
$$

- Magnetic field: $\mathbf{B}=\mathbf{B}^{\prime} B_{0}$, where $B_{0}$ is derived by the Alfvén velocity ${ }^{1}$

$$
B_{0}=u_{0} \sqrt{\rho_{0}}=\sqrt{p_{0}} .
$$

Other normalization constants follow from the previous ones.

- Energy density: $\varepsilon=\varepsilon^{\prime} \varepsilon_{0}$, where

$$
\varepsilon_{0}=p_{0}
$$

- Gravitational constant: $G=G^{\prime} G_{0}$, where

$$
G_{0}=r_{0} u_{0}^{2}
$$

- Spacial derivatives

$$
\nabla=\nabla^{\prime} / r_{0}
$$

With the use of such constants, the Eqs. (3.2) to (3.5) can be written in dimensionless form

$$
\frac{\rho_{0} u_{0}}{r_{0}}\left\{\frac{\partial \rho^{\prime}}{\partial t^{\prime}}+\nabla^{\prime} \cdot\left(\rho^{\prime} \mathbf{u}^{\prime}\right)\right\}=0
$$

${ }^{1}$ The Alfvén velocity is given in Eq. (1.18). Note that in the calculation of the characteristic magnetic field $B_{0}$ we neglect $(4 \pi)^{-1 / 2}$. 


$$
\begin{aligned}
& \frac{\rho_{0} u_{0}^{2}}{r_{0}}\left\{\frac{\partial\left(\rho^{\prime} \mathbf{u}^{\prime}\right)}{\partial t^{\prime}}+\nabla^{\prime} \cdot\left[\rho^{\prime} \mathbf{u}^{\prime} \mathbf{u}^{\prime}+\left(p^{\prime}+\frac{B^{\prime 2}}{8 \pi}\right) I-\frac{\mathbf{B}^{\prime} \mathbf{B}^{\prime}}{4 \pi}\right]\right\}=\frac{\rho_{0} u_{0}^{2}}{r_{0}}\left\{\rho^{\prime} \mathbf{g}^{\prime}\right\} \\
& \frac{B_{0} u_{0}}{r_{0}}\left\{\frac{\partial \mathbf{B}^{\prime}}{\partial t^{\prime}}+\nabla^{\prime} \cdot\left(\mathbf{u}^{\prime} \mathbf{B}^{\prime}-\mathbf{B}^{\prime} \mathbf{u}^{\prime}\right)\right\}=0 \\
& \frac{\varepsilon_{0} u_{0}}{r_{0}}\left\{\frac{\partial \varepsilon^{\prime}}{\partial t^{\prime}}+\nabla^{\prime} \cdot\left[\mathbf{u}^{\prime}\left(\varepsilon^{\prime}+p^{\prime}+\frac{B^{\prime 2}}{8 \pi}\right)-\frac{\left(\mathbf{u}^{\prime} \cdot \mathbf{B}^{\prime}\right) \mathbf{B}^{\prime}}{4 \pi}\right]\right\}=\frac{\varepsilon_{0} u_{0}}{r_{0}}\left\{\rho^{\prime} \mathbf{g}^{\prime} \cdot \mathbf{u}^{\prime}\right\} \\
& \varepsilon^{\prime}=\frac{\rho^{\prime} u^{\prime 2}}{2}+\frac{p^{\prime}}{\gamma-1}+\frac{B^{\prime 2}}{8 \pi}
\end{aligned}
$$

It is immediate to see that the terms outside the curly brackets cancel and we have essentially the same equations as Eqs. (3.2) to (3.5), but with dimensionless variables.

The important result here is that the ideal MHD equations do not depend on the basic parameters of the plasma, i.e., on the characteristic size of the plasma $r_{0}$, on the characteristic velocity of the plasma $u_{0}$, and on the characteristic density of the plasma $\rho_{0}$. Even if these quantities are changed by orders of magnitude, the same MHD equations apply.

\subsubsection{Solving the MHD Equations}

BATS-R-US solves the MHD equations in the dimensionless form [Eqs. (3.16) to (3.19)], but for clarity, we are omitting the prime symbols. The system of Eqs. (3.16) to (3.19) [or, equivalently, Eqs. (3.2) to (3.5)] can be written as

$$
\frac{\partial \mathbf{U}}{\partial t}+(\nabla \cdot \mathbf{F})^{T}=\mathbf{S}
$$

where $\mathbf{U}$ is the vector of conserved quantities

$$
\mathbf{U}=\left(\begin{array}{c}
\rho \\
\rho \mathbf{u} \\
\mathbf{B} \\
\varepsilon
\end{array}\right)
$$

$\mathbf{F}$ is the flux tensor

$$
\mathbf{F}=\left(\begin{array}{c}
\rho \mathbf{u} \\
\rho \mathbf{u} \mathbf{u}+\left(p+\frac{B^{2}}{8 \pi}\right) I-\frac{\mathbf{B} \mathbf{B}}{4 \pi} \\
\mathbf{u} \mathbf{B}-\mathbf{B} \mathbf{u} \\
\mathbf{u}\left(\varepsilon+p+\frac{B^{2}}{8 \pi}\right)-\frac{(\mathbf{u} \cdot \mathbf{B}) \mathbf{B}}{4 \pi}
\end{array}\right)^{T}
$$


and $\mathbf{S}$ is a source vector, i.e., it includes all the terms of the Eqs. (3.2) to (3.5) that cannot be written in the divergence form (the source terms)

$$
\mathbf{S}=\left(\begin{array}{c}
0 \\
\rho \mathbf{g} \\
0 \\
\rho \mathbf{g u}
\end{array}\right)
$$

BATS-R-US solves the MHD equations in the finite-volume approach. Equations are written in the form of Eq. (3.21) and are integrated over the volume $V$, defined by the surface $A$, of the $i$-th cell in the grid

$$
\begin{aligned}
& \int_{\text {cell i }} \frac{\partial \mathbf{U}}{\partial t} \mathrm{~d} V+\int_{\text {cell i }}(\nabla \cdot \mathbf{F})^{T} \mathrm{~d} V=\int_{\text {cell i }} \mathbf{S} \mathrm{d} V \\
& \frac{\partial \mathbf{U}_{i}}{\partial t} V_{i}+\oint \mathbf{F} \cdot \hat{\mathbf{n}} \mathrm{d} A=\mathbf{S}_{i} V_{i},
\end{aligned}
$$

where $\mathbf{U}_{i}$ and $\mathbf{S}_{i}$ denotes the average value of $\mathbf{U}$ and $\mathbf{S}$ in a cell of volume $V_{i}$, and $\hat{\mathbf{n}}$ is the unit vector normal to the area of the cell (Powell et al. 1999). Equation (3.26) is the basis of the finite-volume scheme.

The closed integral in Eq. (3.26) is performed over the surface of the cell and, in the discrete solution, is the sum of the fluxes entering/leaving the six faces of the cubic cell. The exact form of an interface flux is computed using a solver, which is what defines a particular scheme. BATS-R-US has currently several approximate Riemann solvers available, although originally used the upwind Roe-type scheme.

The temporal integration (i.e., the algorithm that updates the numerical solution in time) is performed using either an explicit, implicit or combined explicit-implicit time stepping scheme (details are found in Tóth et al. 2006). In an explicit scheme, a two-level Runge-Kutta scheme is often used. The numerical algorithm has to obey the CourantFriedichs-Lewy condition, or simply the CFL condition, which states that the fluid is not allowed to flow more than one cell within one time-step

$$
\Delta t_{\max }=\frac{\Delta x}{u}
$$

where $\Delta t_{\max }$ is the maximum allowed time-step used to advance the solution in time, $\Delta x$ is the size of the cell, and $u$ is the velocity of the flow. As $u$ and $\Delta x$ have different values in different positions of the grid, the time-step is chosen as the smallest value among the entire grid. In practice, $\Delta t_{\max }$ is multiplied by a number $\mathrm{C}<1$, to make $\Delta t=\mathrm{C} \Delta t_{\max }$ 
smaller (typically $0.5<\mathrm{C}<0.8$ ). The smallest $\Delta t$ is, the more stable is the scheme, but more time-consuming is the simulation. When a single $\Delta t$ is adopted to advance the solution of all the cells in the grid, the computation is said to be in time accurate mode. Contrary, if the time-step is set locally (different values for different positions on the grid), the computation is running on local time stepping. In this case, the convergence of the solution to steady state can be accelerated, but it has to be used with caution, as it may include numerical errors when the solution is far from convergence (Linde 1998).

\subsubsection{Applications of the Code}

BATS-R-US has been widely used to simulate the Earth's magnetosphere (Ridley et al. 2006), the heliosphere (Roussev et al. 2003, Cohen et al. 2007), the outer heliosphere (Linde et al. 1998, Opher et al. 2003; 2004), coronal mass ejections (Manchester et al. 2004, Lugaz et al. 2005), and the magnetosphere of planets (Tóth et al. 2004, Hansen et al. 2005), among others. Its use can be extended to problems for which the MHD equations can be used as a good description for the physical model.

Based on the model developed by Opher et al. (2003; 2004; 2006), we extended the use of BATS-R-US to the study of a magnetized coronal wind of a solar-like star (Chap. 4). The studies further presented in Chaps. 5 to 7 are extensions of the model presented in Chap. 4.

\subsection{Problem Setup}

The setup of the problem is defined in a file created by the user (ModUser.f90), which is read by BATS-R-US during its compilation. In this file, the user provides the details of the physical problem, for example, a description of :

- the simulation grid, including the formulation of a criterion for the AMR;

- the initial condition of the problem, i.e., a description of the physical condition of the system at time $t=0$;

- the boundary conditions used in the integration of the MHD equations; 
- further details of the simulation, such as new variables for the problem, conversion between units, etc.

The specific characteristics of the system, as for instance, the mass of the star, the radius of the star, the intensity of the magnetic field, etc., are specified in another file (PARAM. in), created by the user as well. This second file is read when the code is executed. It also controls all of the functionality of BATS-R-US, which can be accessed by true or false flags. For example, in the PARAM. in file the user defines how and how often the output should be written, if the simulation should resume from a previous run, what is the size of the simulation box, etc. The list of options is extensive and a detailed description of them can be found in the user's manual, distributed with the code.

To illustrate how the problem setup is done, we will describe the setup used to study the magnetized coronal wind of a solar-like star (Chap. 4). Figure 3.2 presents the spherical and Cartesian coordinate systems, which are related to each other according to the following relations

$$
\begin{aligned}
& x=r \sin \theta \cos \varphi, \\
& y=r \sin \theta \sin \varphi,
\end{aligned}
$$

and

$$
z=r \cos \theta
$$

where $r$ is the radial coordinate, $\theta$ the co-latitude, and $\varphi$ is the azimuthal angle measured in the equatorial plane.

\subsubsection{The Adopted Grid}

The grid constructed to study magnetized coronal winds of solar-like stars was set as to provide better resolution around the star and along the equatorial plane, where a current sheet is formed. Figure 3.3 presents three different views of the grid (cut in the $y=0$ plane), where inside each delimited region we indicate the maximum size of the available cell in units of $r_{0}$, where $r_{0}$ is the radius of the star. The grid is Cartesian and the axes $x, y$, and $z$ extend from $-75 r_{0}$ to $75 r_{0}$ (Fig. 3.3a). The center of the star is placed at the origin. 


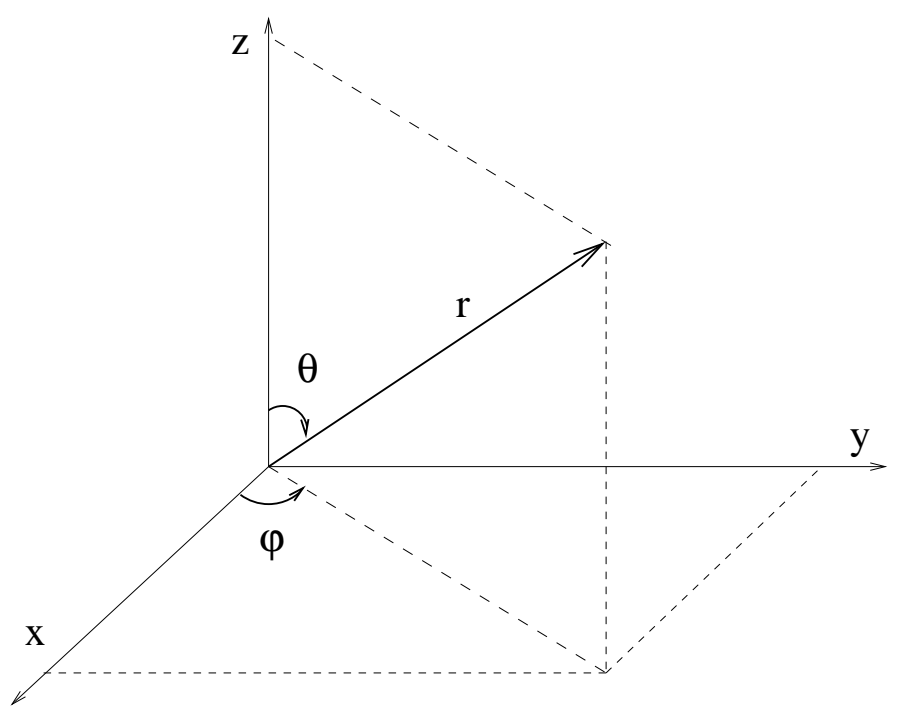

Figure 3.2: Cartesian and spherical coordinate systems.

Initially, the whole simulation domain is refined in five levels. Other five refinement levels are applied next only around the central star and along the equatorial plane. Finally, an additional level is applied around the star. This grid results in $\sim 9.1 \times 10^{6}$ cells in the domain. The smallest cell is located around the central star and has a size of $0.018 r_{0}$ (Fig. 3.3c). The largest cell is located at the corners of the grid and its size is $4.68 r_{0}$ (Fig. 3.3a). The smallest cell size near the current sheet region is $0.036 r_{0}$.

\subsubsection{The Initial Conditions}

To initialize the simulation, it is required to set an initial condition for each cell in the computational domain, i.e., for each cell, the eight quantities $\mathbf{u}, \mathbf{B}, \rho$, and $p$ have to be specified.

At $t=0$, we assume that the grid has a spherically-symmetric hydrodynamical (HD) wind (Parker 1958), as presented in $\$ 1.3 .1$. The purely radial velocity law $\left(\mathbf{u}=u_{r} \hat{\mathbf{r}}\right)$ of such a wind is given by the solution of Eq. (1.5), which is computed in a separate 1D code and, after that, included in the initial setup of BATS-R-US. The only physical possible solution for this wind is the one that becomes supersonic when passing through the critical radius (i.e., when the velocity of the flow equals the sound speed). This solution is dependent solely on the choice of the base temperature of the wind, on the choice of $\gamma$, and on the mass and radius of the star. Figure 3.4a shows the solution of 

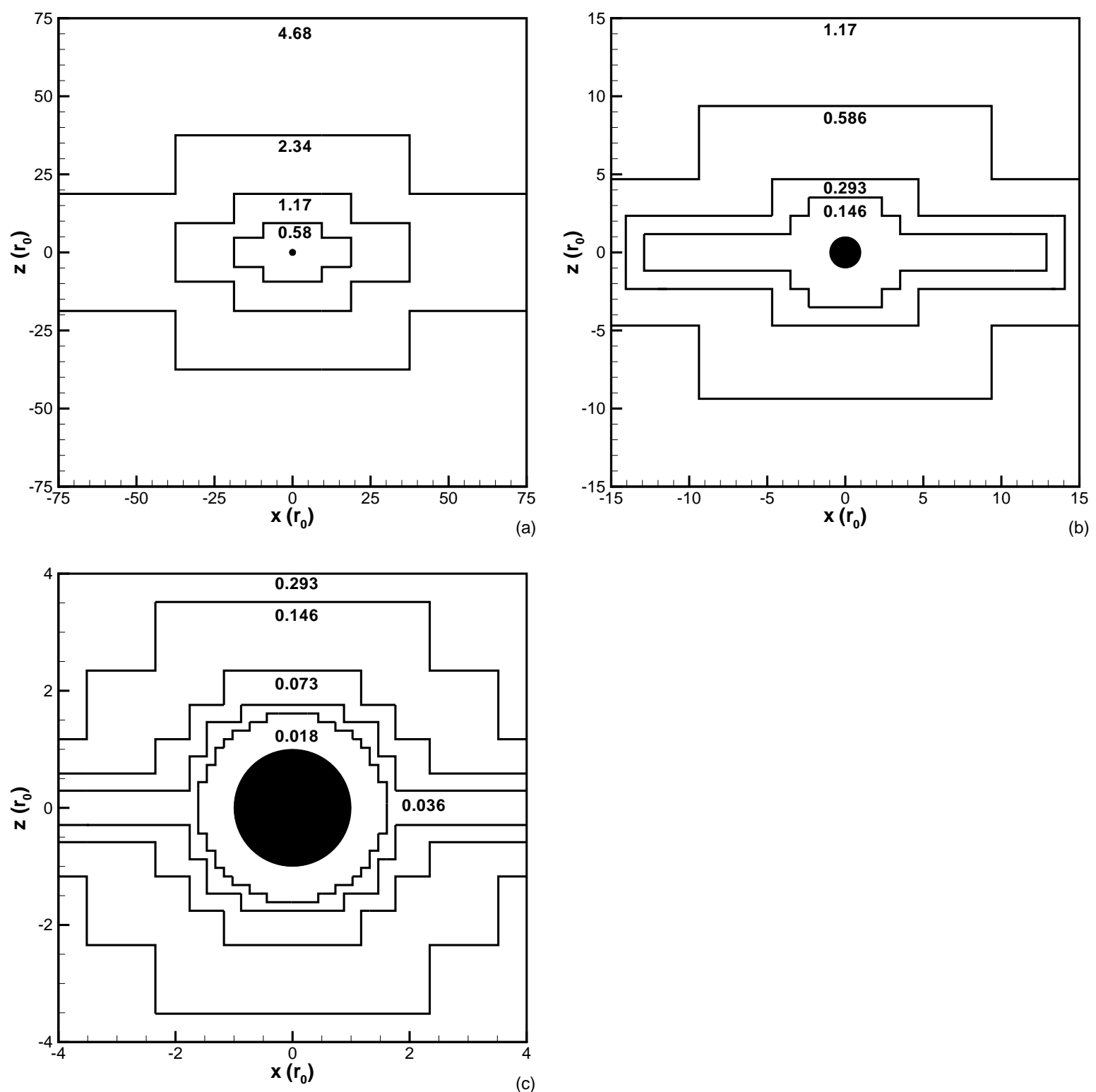

Figure 3.3: Three different views of the grid. Inside each region, we indicate the maximum cell size of the region (in $r_{0}$ units).

an isothermal wind $(\gamma \simeq 1)$ with temperature $T_{0}=1.56 \times 10^{6} \mathrm{~K}$, for a star with $1 \mathrm{M}_{\odot}$ and $r_{0}=1 \mathrm{R}_{\odot}$. Such solution was previously determined in a $1 \mathrm{D}$ model (Fig. 1.10) and inserted in the computational grid of BATS-R-US (Fig. 3.4a). The density profile of the wind is then obtained using conservation of mass of a steady wind [Eq. (1.4)]: $\rho^{-1} \propto u_{r} r^{2}$. We consider ideal gas and, initially, the temperature in the grid obeys the politropic relation $T \propto r^{\gamma-1}$. 

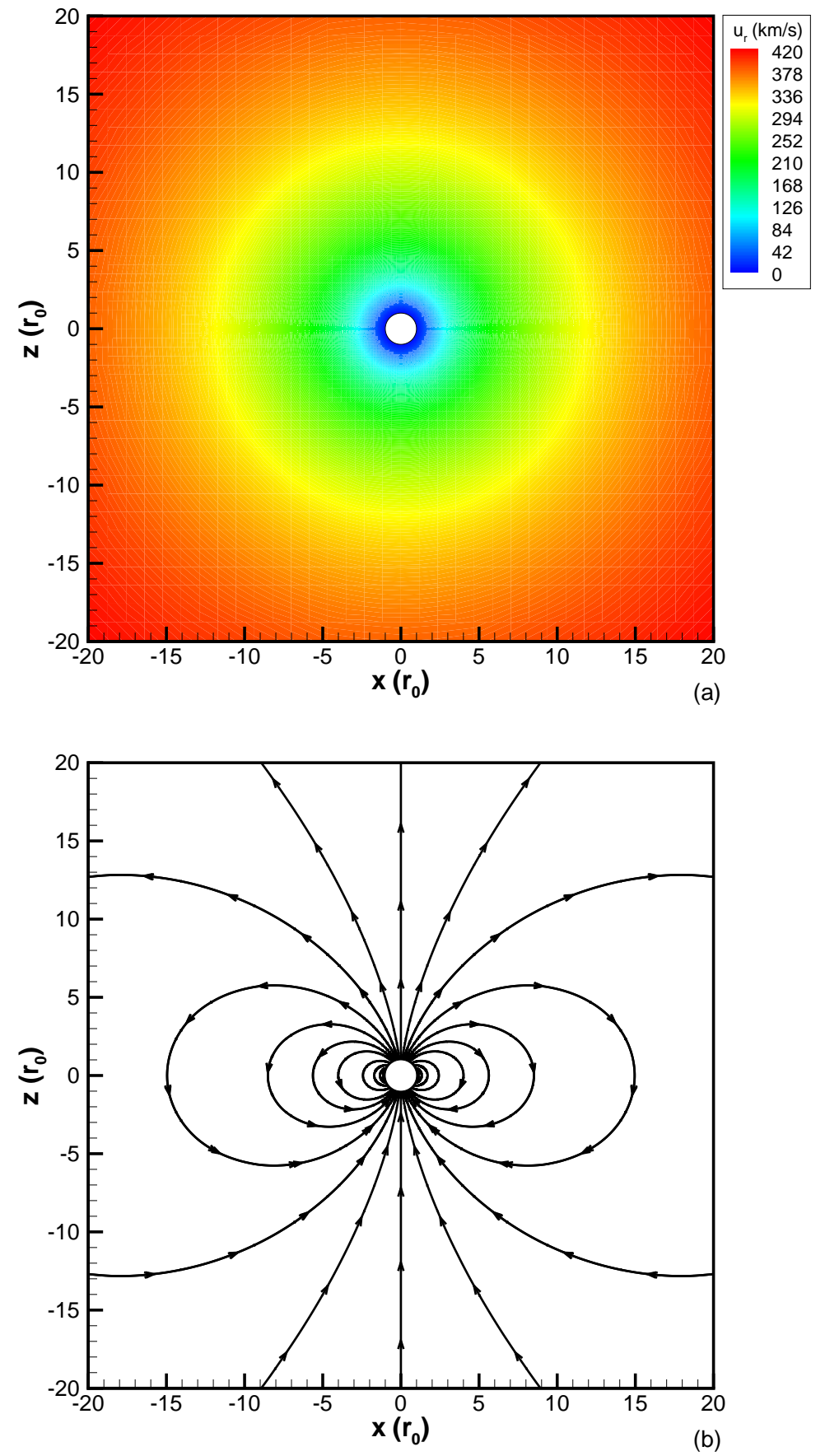

Figure 3.4: The initial conditions adopted in the grid are composed of (a) a HD wind and (b) a dipolar magnetic field in vacuum.

After the spherical wind is set in the grid, a magnetic field is inserted. The simulations are initialized with a bipolar magnetic field configuration, described in spherical 
coordinates $\{r, \theta, \varphi\}$ by

$$
\mathbf{B}=\frac{B_{0} r_{0}^{3}}{r^{3}}\left(\cos \theta, \frac{1}{2} \sin \theta, 0\right)
$$

where $B_{0}$ is the magnetic field intensity at the poles $\left(\theta=0^{\circ}, 180^{\circ}\right)$. Such magnetic configuration is shown in Fig. 3.4b. It should be reminded that this initial configuration of a HD wind plus a dipolar field is not in equilibrium. Thus, when the simulation starts, the stellar magnetic field and the wind velocity interact with each other, modifying the initial configurations set in the grid.

\subsubsection{The Boundary Conditions}

To find the solutions of the MHD equations, the code needs additional information at the boundaries of the system. There are two boundaries in the simulation domain. The inner boundary of the system is the base of the wind, set at $r=r_{0}$. The outer boundary is the edge of the grid. In the simulations that we present next, the outer boundary of the grid is located at $|x|=|y|=|z|=75 r_{0}$. Each boundary is filled with at least two rows of cells that, on the simulations, have the unique role to store the information of the physical conditions at that point. These cells are called ghost cells and they share such informations with adjacent cells that are part of the simulation domain and, for this reason, are called true cells. We tested several different sets of boundary conditions for the simulations we present in the next chapters until we found the set that correctly describes the physical conditions at the inner and outer boundaries. The conditions we use are as follows.

On the inner boundary at $r=r_{0}$, fixed conditions are adopted; particles can freely leave the base of the wind, but no backflow is allowed. In practice, this means that the density $\rho_{0}$ and pressure $p_{0}$ are fixed at $r_{0}$ and their values are set accordingly to the physical problem of interest. The three components of the magnetic filed at $r=r_{0}$ are set as in Eq. (3.31). The three components of the velocity are given as follows: the radial component is set from the thermally driven solution (HD wind), the azimuthal component is entirely due to stellar rotation, and there is no latitudinal component. The star is considered to be rotating as a solid body with its axis of rotation oriented along the $z$ direction, parallel to the magnetic moment vector. It has a period of rotation $P_{0}$, and angular velocity $\Omega=2 \pi / P_{0}$, which gives $u_{\varphi}\left(r_{0}\right)=\Omega r_{0} \sin \theta$. Due to the rotation of the star, the wind that emerges from the star acquires an azimuthal velocity. 
The outer boundary has outflow conditions, i.e., a zero gradient is set to all the primary variables.

\subsubsection{The Steady-State Solution}

As discussed in $§ 3.2 .2$, the initial condition of the simulations is not in equilibrium. This means that the simulation evolves in time, changing both the initial wind velocity and the initial magnetic field configuration. The temporal evolution for the magnetic field will be illustrated in $\S 4.1 .1$. In this section we would like to present how we determine that the system has reached steady state.

When the system is in steady state, all the temporal derivatives in Eqs. (3.2) to (3.5) are null: if one takes a picture of the system in two different instants, the pictures should be the same.

What effectively happens in the simulation is that the conditions imposed at the inner boundary (i.e., at the base of the coronal wind) propagate outwards. The necessary time for the conditions at $r=r_{0}$ to reach the outer boundary depends on the distance the information has to travel and on the velocity of this information. By the time the solution of the wind has propagated outwards, there is no trace of the adopted initial conditions anymore. After that, the system may or may not achieve steady state. In all the simulations we present in the next chapters, except for Chap. 7, steady state is achieved. In practice, if we compare the solution of any of the primary variables of the simulation in two different instants, we note that they are identical. In this case, the simulation is stopped and the steady state solution is found. 


\section{Chapter 4}

\section{D Simulations of Magnetized Winds of Solar-Like Stars}

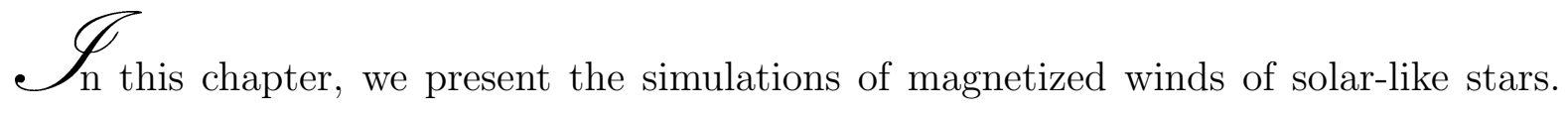
The focus is on stars similar to the Sun: with one solar mass and solar coronal temperatures. This chapter has a didactic importance, as it is here where we present the global characteristics of a coronal magnetized wind, that will serve as basis for the next chapters. The results presented here were published in the Astrophysical Journal, 2009, 699, 441 (Vidotto et al. 2009a).

We aim at investigating in detail the behavior of the long-lived coronal features in solar-like stellar winds (such as the ones presented in Fig. 1.3). In the absence of a magnetic field, a non-rotating corona expands spherically (Parker 1958, Velli 1994). A different picture, however, is expected if the magnetic energy density at the base of the corona is at least of the same amount of the thermal energy density. We investigate the influence of the magnetic field in solar-like stellar winds with different plasma- $\beta$ (the ratio between thermal and magnetic energy densities). We solve the fully 3D MHD equations with the temporal evolution of the energy equation. Therefore, the topology of the magnetic field is not restricted and the steady state arises from the dynamical interplay of the outflow and the magnetic field. Also, meridional flows arise naturally in the system due to the presence of the Lorentz force. We neglect stellar rotation in this chapter. The results presented here are thus valid for non-rotators or slow rotators in the region where the toroidal component of the magnetic field is still much smaller than the 
poloidal component.

The numerical model we use was presented in Chap. 3. We adopted a star with $M_{\star}=1 M_{\odot}$ and $r_{0}=1 R_{\odot}$. The wind consists of a totally ionized plasma of hydrogen (i.e., $\mu=0.5$ ) and has a coronal base temperature of $T_{0}=1.56 \times 10^{6} \mathrm{~K}$. The adopted grid was described in $\S 3.2 .1$, with the initial and boundary conditions presented in $§ 3.2 .2$ and $§ 3.2 .3$, respectively.

Table 4.1 presents the parameters used in the simulations. S00 is a purely HD simulation included for comparison purposes. The simulations are divided in three sets. The first set of simulations, composed by simulations S01 to S05, aims at investigating the effect of the magnetic energy density on the wind. We increased $B_{0}$ from 1 to $20 \mathrm{G}$ while maintaining the other initial values fixed. As a consequence, $\beta_{0}$, the ratio between the surface thermal pressure $p_{0}$ and the magnetic pressure evaluated at the pole

$$
\beta_{0}=\frac{8 \pi p_{0}}{B_{0}^{2}}
$$

decreases from 1 to $1 / 400$. S01 is similar to the case studied by Pneuman and Kopp (1971) with $\beta_{0}=1$. The initial HD wind is the same for all the simulations of the first set $\left(\rho_{0}=\rho_{c}=1.544 \times 10^{-16} \mathrm{~g} \mathrm{~cm}^{-3}, T_{0}=1.56 \times 10^{6} \mathrm{~K}\right.$, as can be seen in Table 4.1). In the second set of simulations, from simulations S06 to S09, $B_{0}$ is fixed at $20 \mathrm{G}$ and we vary $\rho_{0}$ from 1 to $400 \rho_{c}$, thus increasing $\beta_{0}$ back to 1 in S09. In both sets of simulations, we adopted the value $\gamma=1.01$. We then consider a third set of simulations, where parameters similar to the first and second sets are used, but we adopt $\gamma=1.1$.

\subsection{The First Set of Simulations}

The Lorentz force $\mathbf{F}_{\mathbf{B}}$ acting on the flow is given by the difference between the magnetic tension $\mathcal{T}_{\mathbf{B}}$ and the gradient of the magnetic pressure $P_{B}(\S 2.2 .5)$. As the magnetic field lines are more curved at low latitudes, the tension becomes more important as one approaches the equatorial plane. Hence, opening a bipolar field structure at low latitudes, where lines are more curved, is more difficult than opening a bipolar field topology at high latitudes. As both $\mathcal{T}_{\mathbf{B}}$ and $\nabla P_{B}$ are latitude-dependent, so is $\mathbf{F}_{\mathbf{B}}$. We therefore expect from the interaction of the outflow and the magnetic field a latitude-dependent wind. 
Table 4.1: The complete set of simulations for solar-like stars. The columns represent, respectively: the name of the simulation, the temperature of the wind at the base of the corona, the density at $r_{0}$, the surface magnetic field intensity at the pole, the plasma- $\beta$ evaluated at the pole, and the value of $\gamma$ adopted.

\begin{tabular}{cccccc}
\hline \hline Name & $T_{0}$ & $\rho_{0}$ & $B_{0}$ & $\beta_{0}$ & $\gamma$ \\
- & $\left(10^{6} \mathrm{~K}\right)$ & $\left(\rho_{c}{ }^{*}\right)$ & $(\mathrm{G})$ & - & - \\
\hline $\mathrm{S} 00$ & 1.56 & 1 & 0 & $\infty$ & 1.01 \\
\hline \multicolumn{5}{c}{ First Set of Simulations } \\
\hline S01 & 1.56 & 1 & 1 & 1 & 1.01 \\
$\mathrm{~S} 02$ & 1.56 & 1 & 5 & $1 / 25$ & 1.01 \\
$\mathrm{~S} 03$ & 1.56 & 1 & 10 & $1 / 100$ & 1.01 \\
$\mathrm{~S} 04$ & 1.56 & 1 & 15 & $1 / 225$ & 1.01 \\
$\mathrm{~S} 05$ & 1.56 & 1 & 20 & $1 / 400$ & 1.01 \\
\hline \multicolumn{7}{c}{ Second Set of Simulations } \\
\hline S06 & 1.56 & 1.8 & 20 & $1 / 225$ & 1.01 \\
S07 & 1.56 & 4 & 20 & $1 / 100$ & 1.01 \\
S08 & 1.56 & 16 & 20 & $1 / 25$ & 1.01 \\
S09 & 1.56 & 400 & 20 & 1 & 1.01 \\
\hline \multicolumn{7}{c}{ Third Set of Simulations } \\
\hline S01b & 1.56 & 1 & 1 & 1 & 1.1 \\
S02b & 1.56 & 1 & 5 & $1 / 25$ & 1.1 \\
S08c & 1.56 & 16 & 20 & $1 / 25$ & 1.1 \\
S09c & 1.56 & 400 & 20 & 1 & 1.1 \\
\hline${ }^{*} \rho_{c}=1.544 \times 10^{-16}$ g cm ${ }^{-3}$ & & &
\end{tabular}

\subsubsection{Evolution to Steady State}

Figures $4.1 \mathrm{a}$ and $4.1 \mathrm{~b}$ present the temporal evolution of the magnetic field lines from iteration $N=1$ to $N=20,000$. The plots presented are cuts in the meridional plane $(y=0)$, for S03. Figure 4.1a shows that, at high latitudes, field lines tend to become open. Figure $4.1 \mathrm{~b}$ shows the initial stretching of the lines emerging from low latitudes along the equator. This temporal evolution of the magnetic field lines was common to all runs.

As the simulation evolves in time, the magnetic field lines reconnect in the equato- 


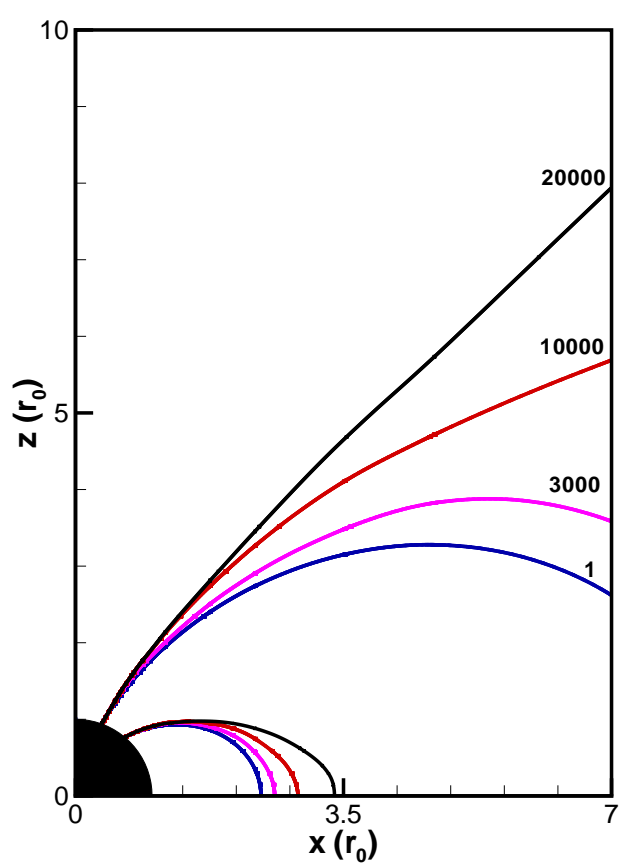

(a)

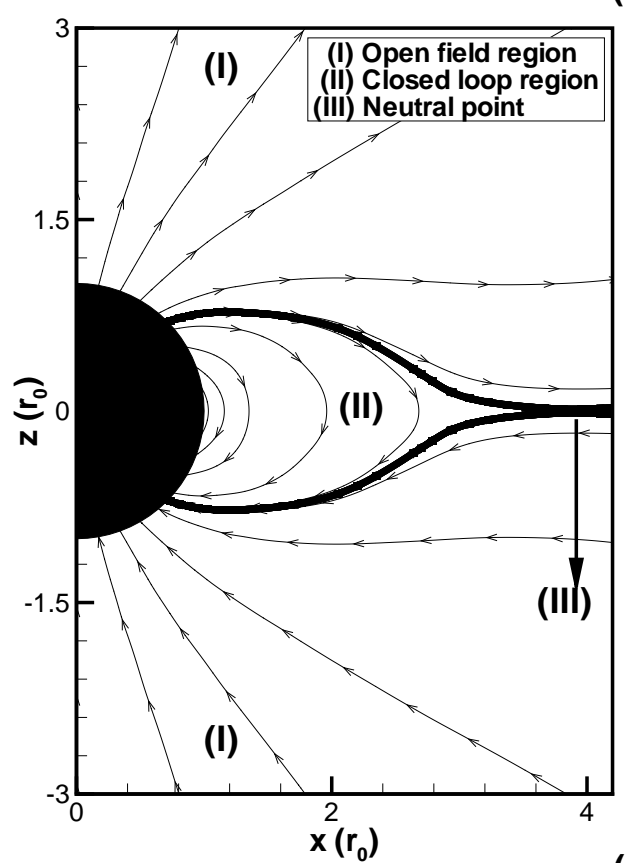

(c)

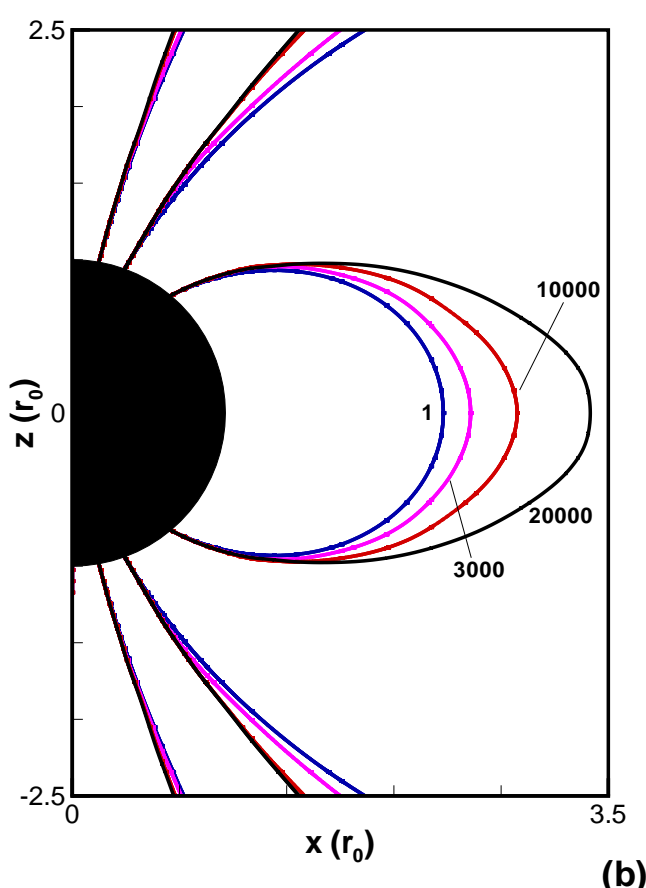

(b)

Figure 4.1: (a) and (b): Evolution of the magnetic field lines for S03. The color of each streamline represents a given iteration, ranging from $N=1$ ( $t=0$, blue) to 20,000 ( $t=7 \mathrm{~h}$, black). At $N=1$, the field is a dipole. The steady state is achieved at $N=148,000(t=51 \mathrm{~h})$. (c): Final steady-state configuration of the magnetic lines: (I) represents the zone of open field lines; (II) the zone of closed field lines; (III) the location of the neutral point. The thick line represents the position of the current sheet formed. 
rial plane. The steady-state configuration (Fig. 4.1c) consists of a formation of a bicomponent wind. This configuration is composed by a zone of open magnetic field lines (I) coexisting with a zone of closed loops anchored on the star (II), also called helmet streamer. At the top of the closed loops lies the neutral point of cusp-type (III): approaching the neutral point from inside the closed loops, the magnetic field goes to zero. Beyond the neutral point, along the equatorial plane, a current sheet is formed (thick line in Fig. 4.1c). The zone of closed field lines is located at low latitudes from $\sim-45^{\circ}$ to $45^{\circ}$ and extends up to the neutral point. Beyond the neutral point, the zone of open field lines fills all the volume. Figure 4.2 shows a $3 \mathrm{D}$ view of the final magnetic configuration.

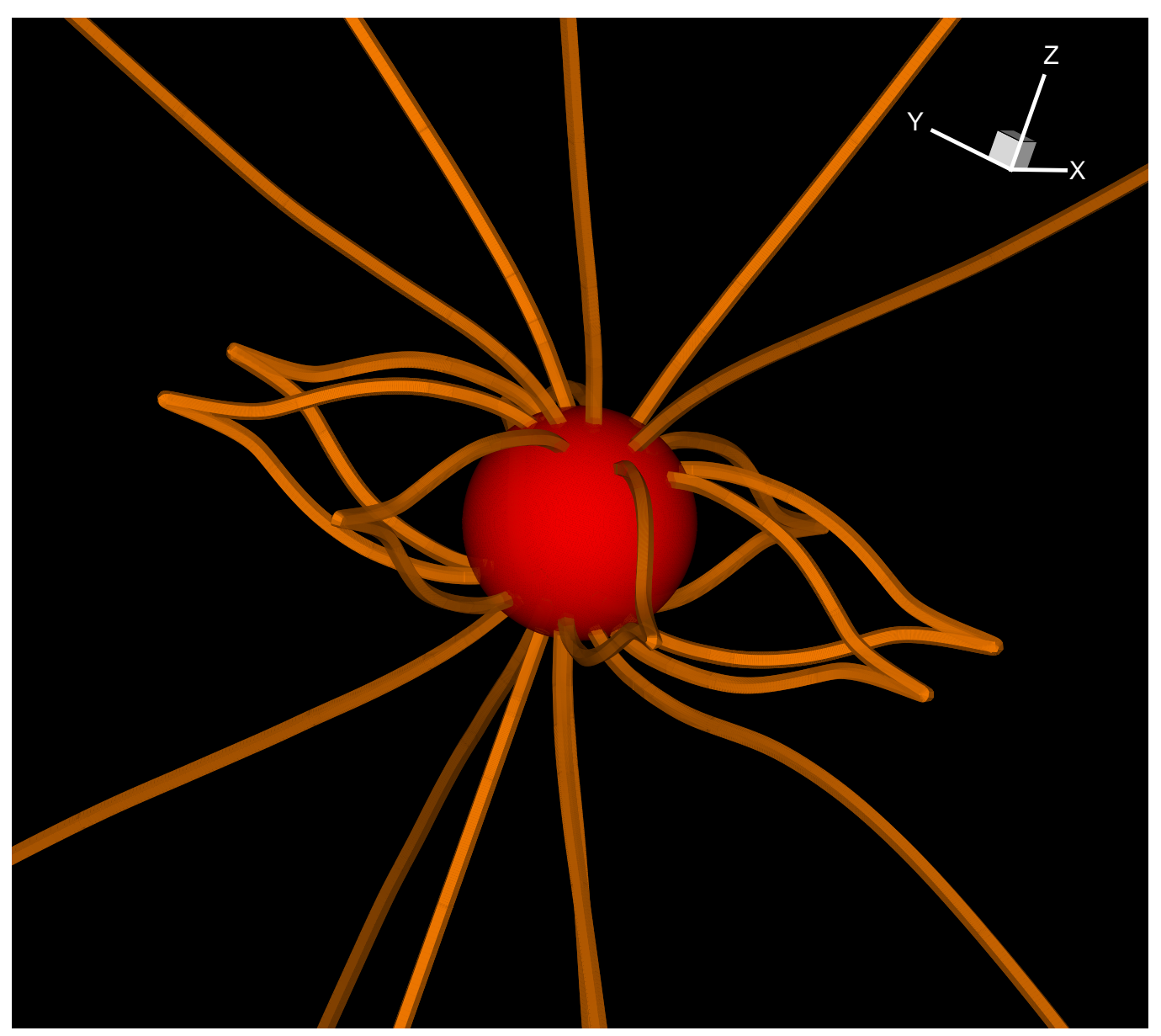

Figure 4.2: Three-dimensional view of the steady state for S03. 


\subsubsection{Steady-State Wind Profile}

Figure 4.3 shows the total velocity of the wind in the meridional plane for the first set of simulations. The magnetic field lines are the black lines and the Alfvén surface is indicated by the white line. In the Alfvén surface, the velocity of the flow $|\mathbf{u}|$ equals the Alfvén velocity $v_{A}$ [Eq. (1.18)]. It can be seen that the increase of $B_{0}$ leads to faster winds.

Although the five panels in Fig. 4.3 present similar structures, they are quantitatively different. The wind is not spherical; higher velocities are achieved at high latitudes. The higher $B_{0}$ is, the higher is the departure from spherical symmetry and the higher is the total velocity of the wind.

The bimodality of the wind is due to the nature of the magnetic force. A purely HD (non-rotating) wind is spherically symmetric but, in the MHD case, this symmetry is lost because the magnetic force has a latitudinal component.

The solar wind has an observed bimodal velocity that, in solar minima, ranges from 400 to $800 \mathrm{~km} \mathrm{~s}^{-1}$ at $1 \mathrm{AU}$ (McComas et al. 1998). Although our model does not make use of magnetograms and thermal latitudinal heating as more realistic solar models such as Cohen et al. (2007) and Liu et al. (2008) do, it is interesting to compare S01 to their results. In Liu et al.'s (2008) model, at $r=4.5 r_{0}$, the highest velocity of $\sim 440 \mathrm{~km} \mathrm{~s}^{-1}$ is achieved at the polar axis $\left(\theta=0^{\circ}\right)$, while in the mid-latitudes $\left(\theta \sim 45^{\circ}\right)$, this value is decreased to $\sim 230 \mathrm{~km} \mathrm{~s}^{-1}$. For $\mathrm{S} 01$, the same positions lead to $201 \mathrm{~km} \mathrm{~s}^{-1}$ and $190 \mathrm{~km} \mathrm{~s}^{-1}$, respectively. In our models, we are not treating the presence of waves and damping that is responsible for the latitude dependence that is observed in the solar wind. However, it is interesting that, assuming an initially spherical wind interacting with the magnetic field, at steady state, we obtain a latitude-dependent wind, although not as dramatic as in the Sun.

Figure 4.4 presents a radial cut at $r=4.5 r_{0}$ for cases S00 and S01. There is a range of angles, $\sim 30^{\circ}$ above and below the equator, where the MHD wind (solid line) is slower compared to the HD model (S00, double-dot-dashed line). This deceleration is a consequence of the magnetic tension that is stronger near the equator. At high latitudes, the gradient of both thermal and magnetic pressures is responsible for driving the wind. Figure 4.4 also presents the analytical result for a non-magnetized wind (dashed line) for comparison purposes. The width of the decrease seen in Fig. 4.4 for the S01 curve is 

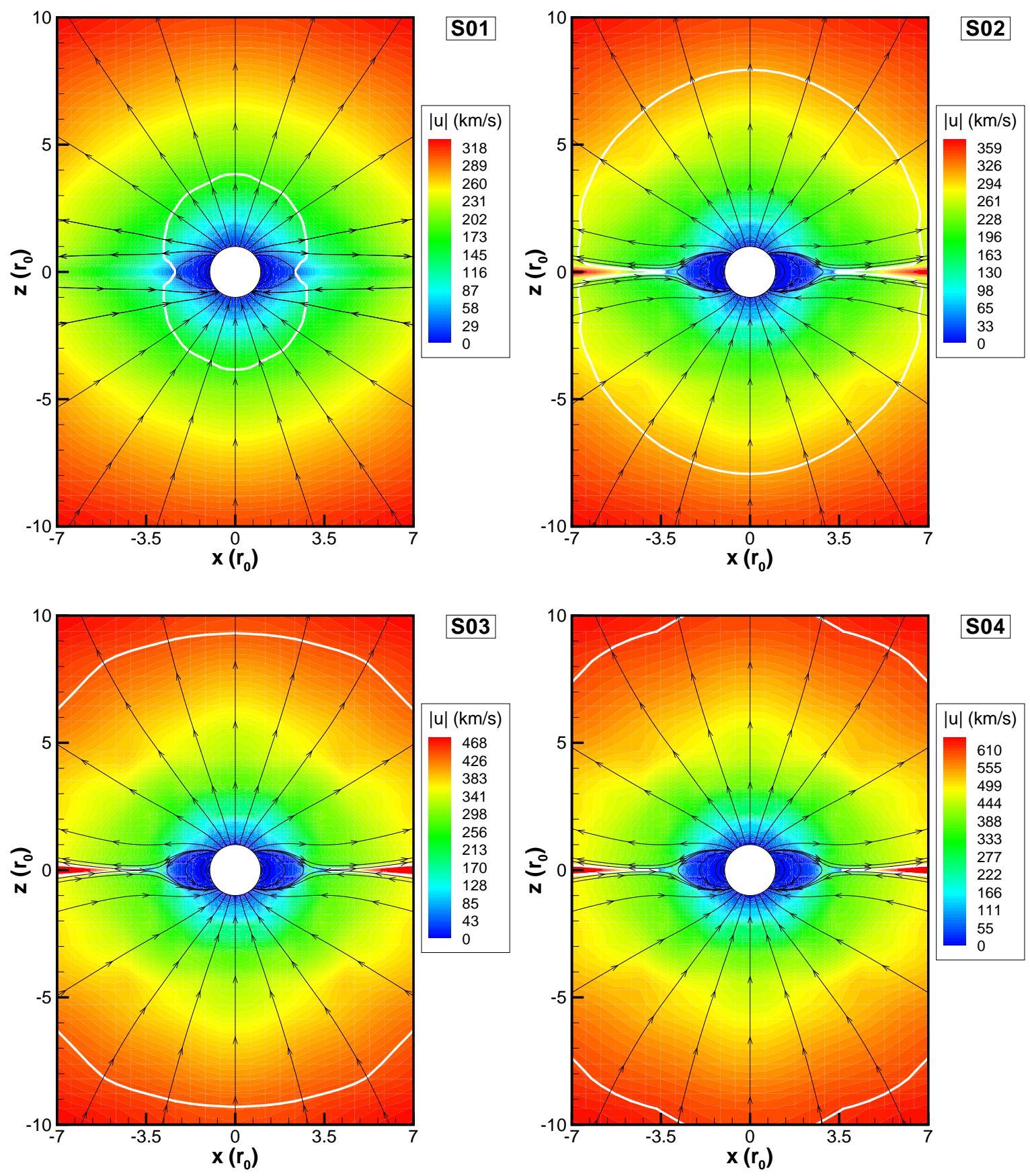

Figure 4.3: Meridional cuts of the steady-state configurations for simulations S01 to S05. Black streamlines represent the final configuration of the magnetic field. Contour plots of the velocity of the flow are shown in the background. The white line is the Alfvén surface. Continues in the next page. 


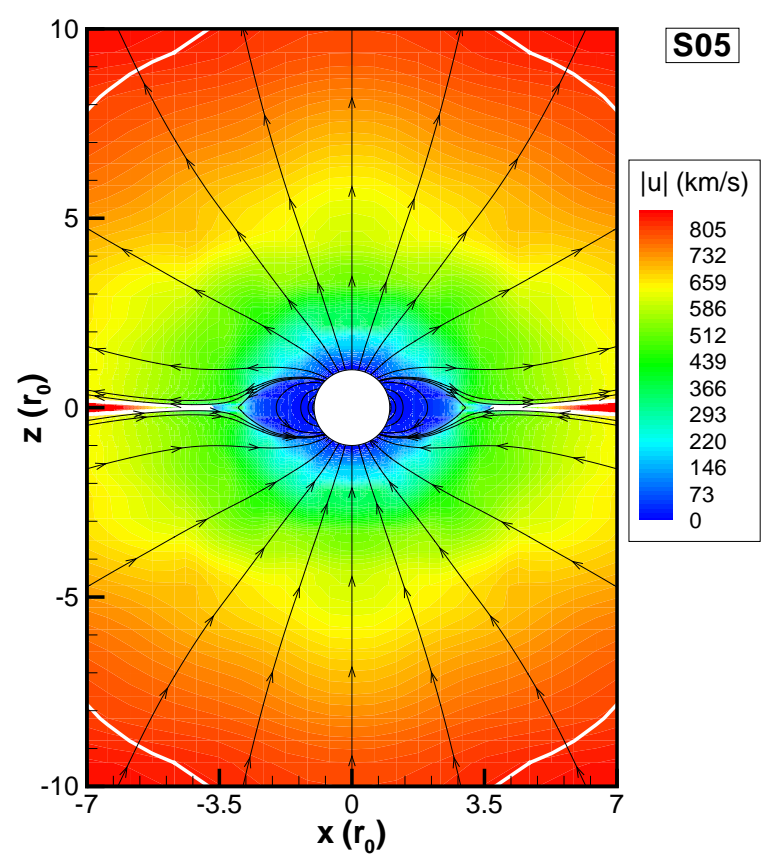

Figure 4.3: Cont.

dependent on the grid resolution (Opher et al. 2004).

Figure 4.5 presents the radial cuts for the remaining simulations of the first set from $\theta=0^{\circ}$ to $90^{\circ}$. All the curves present smaller velocities at the equator $\left(\theta=90^{\circ}\right)$ and higher velocities at high latitudes. The difference in velocities increases as $B_{0}$ is increased (see Table 4.2).

Another consequence of the latitudinal dependence of the Lorentz force, the mass-loss

Table 4.2: Co-latitude dependence of the radial velocity $u_{r}$ at $r=4.5 r_{0}$. The columns represent, respectively: the name of the simulation, $u_{r}$ evaluated at $\theta=18^{\circ}, u_{r}$ evaluated at $\theta=45^{\circ}$, and the difference $\Delta u_{r}=u_{r}\left(\theta=18^{\circ}\right)-u_{r}\left(\theta=45^{\circ}\right)$.

\begin{tabular}{cccc}
\hline \hline Name & $\begin{array}{c}u_{r}\left(\theta=18^{\circ}\right) \\
-\end{array}$ & $\begin{array}{c}u_{r}\left(\theta=45^{\circ}\right) \\
\left(\mathrm{km} \mathrm{s}^{-1}\right)\end{array}$ & $\begin{array}{c}\Delta u_{r} \\
\left(\mathrm{~km} \mathrm{~s}^{-1}\right)\end{array}$ \\
\hline S01 & 200 & 189 & 11 \\
S02 & 243 & 224 & 19 \\
S03 & 329 & 295 & 34 \\
S04 & 456 & 400 & 56 \\
S05 & 610 & 528 & 82 \\
\hline
\end{tabular}




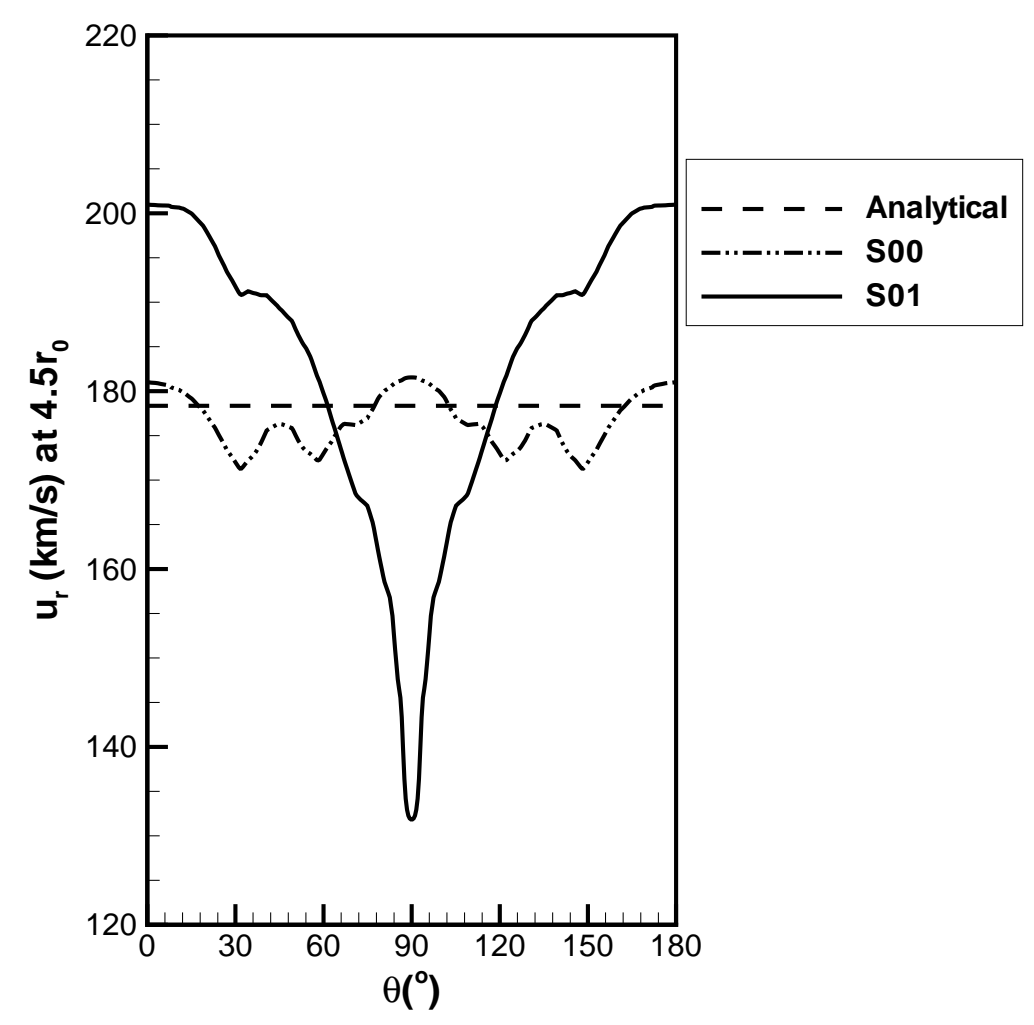

Figure 4.4: Radial velocity of the wind as a function of $\theta$ at $r=4.5 r_{0}$, for S00 (double-dotdashed line), S01 (solid line) and analytical solution of a non-magnetized wind (dashed line).

rate per unit solid angle of a steady wind

$$
\dot{m}(\theta)=r^{2} \rho(\theta) u_{r}(\theta)
$$

is also latitude-dependent. According to Eq. (3.2), $\dot{m}(\theta)$ is constant if there is no variation in $\theta(\partial / \partial \theta=0)$. In our simulations, this is only verified far from the central star. Table 4.3 presents the calculated $\dot{m}(\theta)$ for two given co-latitudes of the steady-state wind, when it has already reached constant values. $\dot{m}(\theta)$ at the pole is smaller compared to other angles, despite the increase of the radial velocity. The lower value of $\dot{m}(\theta)$ at the pole is due to matter carried from high latitudes to low latitudes (§4.1.3). As the latitudinal increase of density is higher than the corresponding decrease in the radial velocity, $\dot{m}(\theta)$ is higher at low latitudes. 


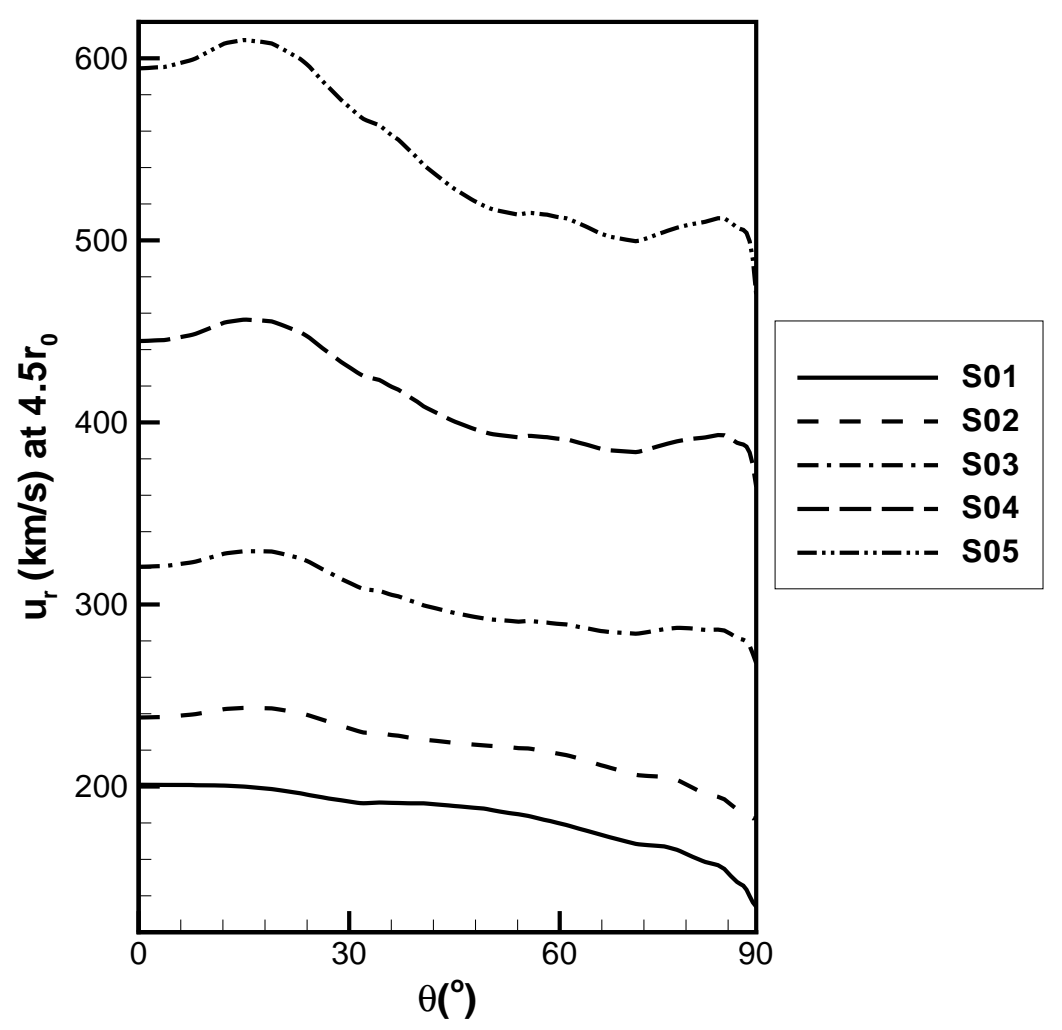

Figure 4.5: Same as Fig. 4.4, but for S01 to S05, from $\theta=0^{\circ}$ to $\theta=90^{\circ}$.

Table 4.3: Mass-loss rate $\dot{m}$ per unit solid angle of the outflow. The columns represent, respectively: the name of the simulation, $\dot{m}$ evaluated at $\theta=0^{\circ}$, and $\dot{m}$ evaluated at $\theta=45^{\circ}$.

\begin{tabular}{|c|c|c|}
\hline $\begin{array}{c}\text { Name } \\
-\end{array}$ & $\begin{array}{c}\dot{m}\left(\theta=0^{\circ}\right) \\
\left(10^{-15} \mathrm{M}_{\odot} \mathrm{yr}^{-1}\right)\end{array}$ & $\begin{array}{c}\dot{m}\left(\theta=45^{\circ}\right) \\
\left(10^{-15} \mathrm{M}_{\odot} \mathrm{yr}^{-1}\right)\end{array}$ \\
\hline S01 & 5.7 & 5.8 \\
\hline S02 & 5.1 & 5.7 \\
\hline S03 & 6.0 & 6.6 \\
\hline S04 & 7.1 & 7.5 \\
\hline S05 & 8.2 & 8.7 \\
\hline
\end{tabular}

\subsubsection{Meridional Flows}

The component of the magnetic force in the $\theta$ direction gives rise to meridional flows, bringing matter from both hemispheres towards the equator. This causes a density enhancement along the equatorial plane. 
Figure 4.6 presents the meridional velocity, $u_{\theta}$, for S01 to S05 at steady state. $u_{\theta}$ increases with $B_{0}$, ranging from a maximum value of $28 \mathrm{~km} \mathrm{~s}^{-1}$ in $\mathrm{S} 01\left(B_{0}=1 \mathrm{G}\right)$ to $339 \mathrm{~km} \mathrm{~s}^{-1}$ in S05 $\left(B_{0}=20 \mathrm{G}\right)$. The last panel of Fig. 4.6 presents the flux of matter in the $\theta$ direction, $\rho u_{\theta}$, at $4.5 r_{0}$. It can be seen that, as the magnetic field increases, there is an increase of the meridional flux of matter.

\subsubsection{The Neutral Point}

The meridional flows are also responsible for compressing lines with opposite polarities along the equatorial plane and due to reconnection, a current sheet is formed. In our simulations, the reconnection is happening due to numerical resistivity. In order to minimize the effects of numerical resistivity, we refined the grid along the equatorial plane. Ultimately, a more realistic reconnection should be included in the global 3D MHD simulations, such as done by Kuznetsova et al. (2007). The reconnection leads to the emergence of a faster wind in the equatorial plane, beyond the reconnection point (Fig. 4.3). Such wind is an artifact of our model, and a similar behavior was also observed by Roussev et al. (2003).

The location of the neutral point $r_{N}$ is given by the requirement that the total pressure (magnetic plus thermal) at the tip of the cusp of closed loops is continuous

$$
p\left(r_{N}^{+}\right)-p\left(r_{N}^{-}\right)=\frac{B\left(r_{N}\right)^{2}}{8 \pi}
$$

where $p\left(r_{N}^{+}\right)$is the thermal pressure evaluated immediately after $r_{N}$ and $p\left(r_{N}^{-}\right)$immediately before $r_{N}$ (Pneuman and Kopp 1971). Along a magnetic streamline, the magnetic force is null. Assuming an isothermal atmosphere, the energy equation along a magnetic streamline reduces to

$$
\frac{u^{2}}{2}+a^{2} \ln \frac{p}{p_{0}}+g r=\frac{u_{0}^{2}}{2}+g_{0} r_{0},
$$

where $a$ denotes the isothermal sound speed and the use of the subscript " 0 " indicates that the quantity is evaluated at the base of the wind. Equation (4.3) can be written as

$$
p\left(r_{N}^{+}\right)-p\left(r_{N}^{-}\right) \simeq p\left(r_{N}^{+}\right)\left[\exp \frac{1}{2}\left(\frac{u\left(r_{N}\right)}{a}\right)^{2}-1\right] \simeq p\left(r_{N}^{+}\right) \frac{u\left(r_{N}\right)^{2}}{2 a^{2}}
$$

where we neglected the initial velocity of the flow in comparison with the velocity of the flow at the neutral point, $u\left(r_{N}\right)$, and assumed $u\left(r_{N}\right) \ll a$. Under these considerations, 

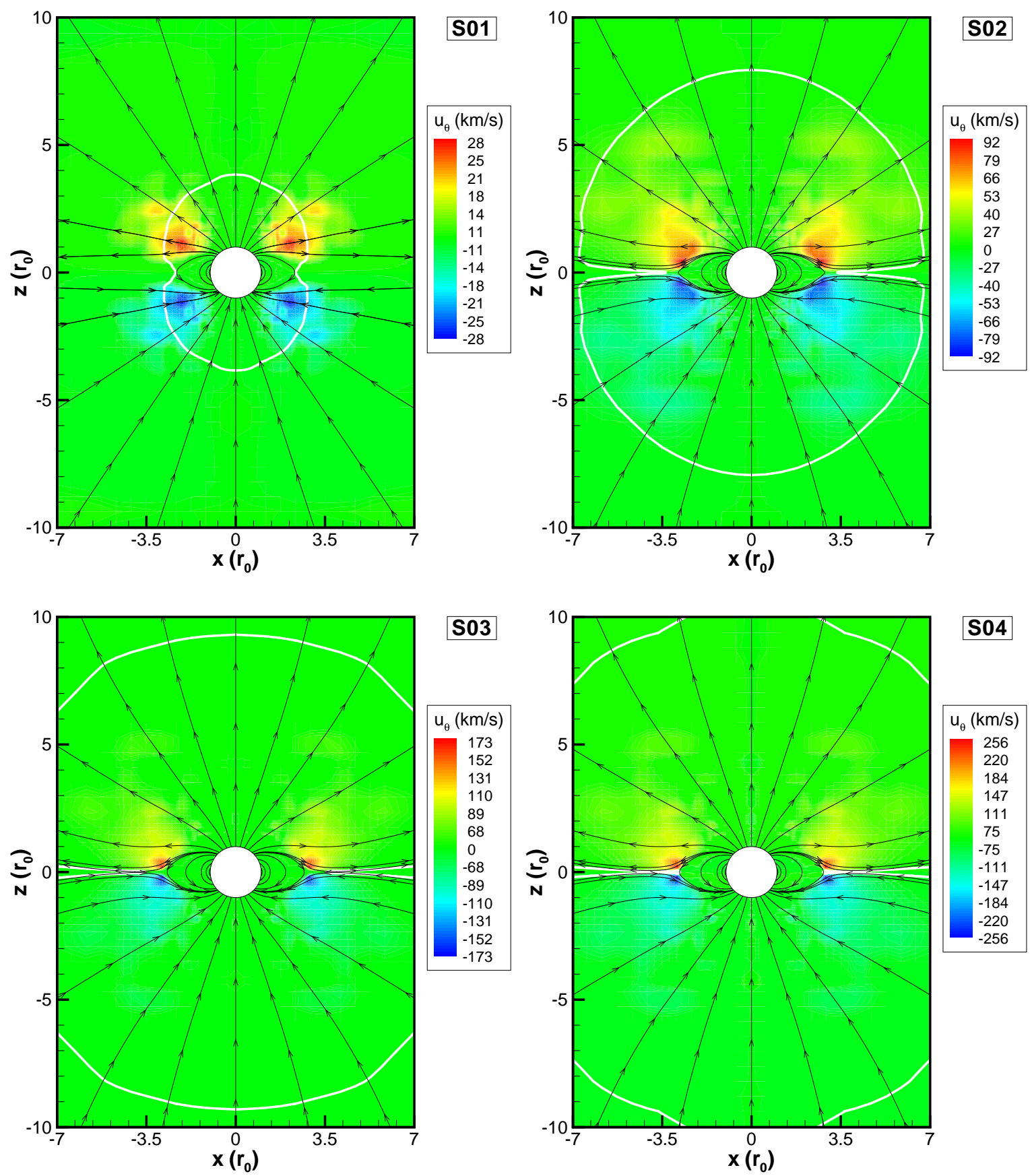

Figure 4.6: Meridional velocities $u_{\theta}$ for the steady-state configurations of simulations S01 to S05. Black streamlines are the magnetic field lines. The white line is the Alfvén surface. Last panel in the next page: The flux of matter in the $\theta$ direction, $\rho u_{\theta}$, for $S 01$ to $S 05$ at $r=4.5 r_{0}$. Continues in the next page. 

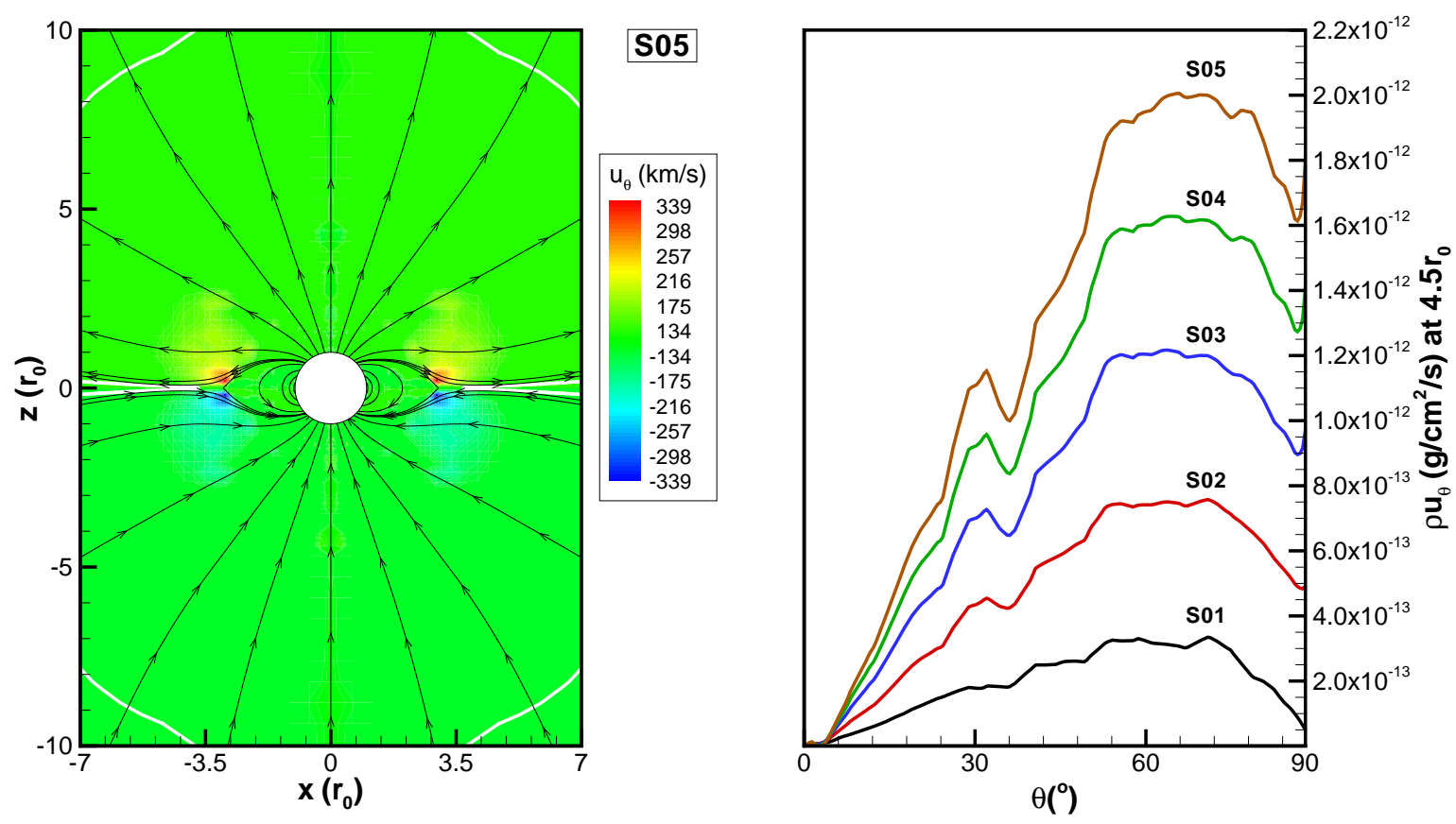

Figure 4.6: Cont.

Eqs. (4.3) and (4.5) lead to

$$
\frac{B\left(r_{N}\right)^{2}}{8 \pi} \simeq p\left(r_{N}^{+}\right) \frac{u\left(r_{N}\right)^{2}}{2 a^{2}} \simeq \frac{1}{2} \rho\left(r_{N}^{+}\right) u\left(r_{N}\right)^{2},
$$

implying that at the neutral point

$$
u\left(r_{N}\right) \simeq v_{A}\left(r_{N}\right)
$$

where we have used Eq. (1.18) for $v_{A}$, i.e., the neutral point lies on the Alfvén surface.

Figure 4.6 presents the Alfvén surface (white line), where it can be seen that the above result is confirmed by our simulations. In Table 4.4 the position of the neutral point $r_{N}$ is given for each of the simulations of the first set. The cell size at the current sheet is $0.036 r_{0}$, so the numerical error associated with $r_{N}$ is $\pm 0.018 r_{0}$ (half the size of the cell). $r_{N}$ increases as $B_{0}$ is increased, i.e., the dead-zone (zone of closed loops) is larger for larger $B_{0}$. As $B_{0}$ is increased from $5 \mathrm{G}$ to $20 \mathrm{G}, r_{N}$ moves from $3.68 r_{0}$ to $4.12 r_{0}$, which is explained due to the fact that the Alfvén surface moves farther from the star as $B_{0}$ is increased. S01 does not present reconnection.

Inside the closed field line region, particles are trapped. This is evidenced in Fig. 4.7, where we plot the magnetic field lines and the vectors of the flow velocity. As can be seen, 
Table 4.4: Location of the neutral point, $r_{N}$, along the equatorial plane as a function of $B_{0}$ for the first set of simulations.

\begin{tabular}{ccc}
\hline \hline Name & $B_{0}$ & $r_{N}$ \\
- & $(\mathrm{G})$ & $\left(r_{0}\right)$ \\
\hline $\mathrm{S} 02$ & 5 & 3.68 \\
$\mathrm{~S} 03$ & 10 & 3.90 \\
$\mathrm{~S} 04$ & 15 & 4.01 \\
$\mathrm{~S} 05$ & 20 & 4.12 \\
\hline
\end{tabular}

if a given particle emerges inside the closed loop zone, it will remain there, because its velocity is not high enough to escape the effective potential well created by the magnetic tension and gravitational attraction force.

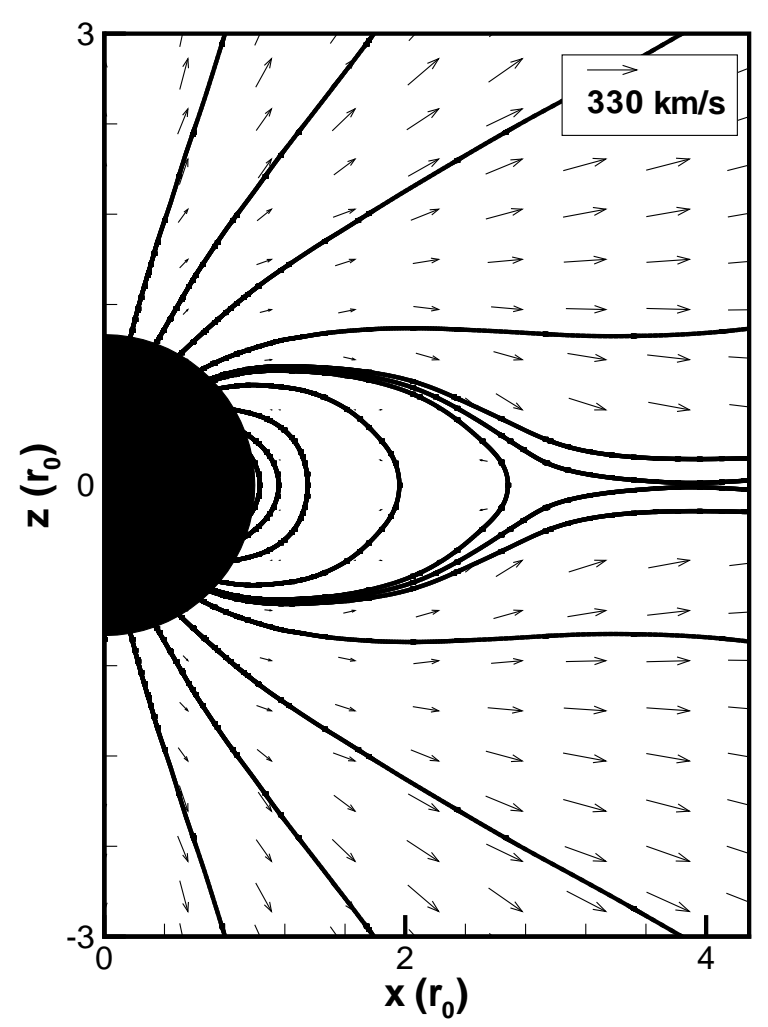

Figure 4.7: Trapping of particles inside the closed loop region for S03. Thick lines represent the magnetic field lines and vectors represent the flow velocity, both in the meridional plane $(y=0)$. 


\subsubsection{Energetics}

In order to verify if energy is conserved in the simulations, we would like to estimate the contribution of the different energies to the total energy content of the system.

The temperature $T$ of the steady-state coronal wind increases with $B_{0}$. Figure 4.8 presents the temperature profiles along the polar axis for the cases of the first set of simulations (in steady state). For higher $B_{0}, T$ increases near the base of the wind and it drops slowly for larger radii. For S05, the increase in the temperature is maximum among all the cases, reaching $\simeq 5.9 \mathrm{MK}$ around $6 r_{0}$. In the solar wind, however, the temperature profile is not flat (Matthaeus et al. 2006), because the bulk of the internal energy deposition occurs near the Sun.

For steady state, conservation of total energy flux is given by

$$
\nabla \cdot \mathbf{q}=0
$$

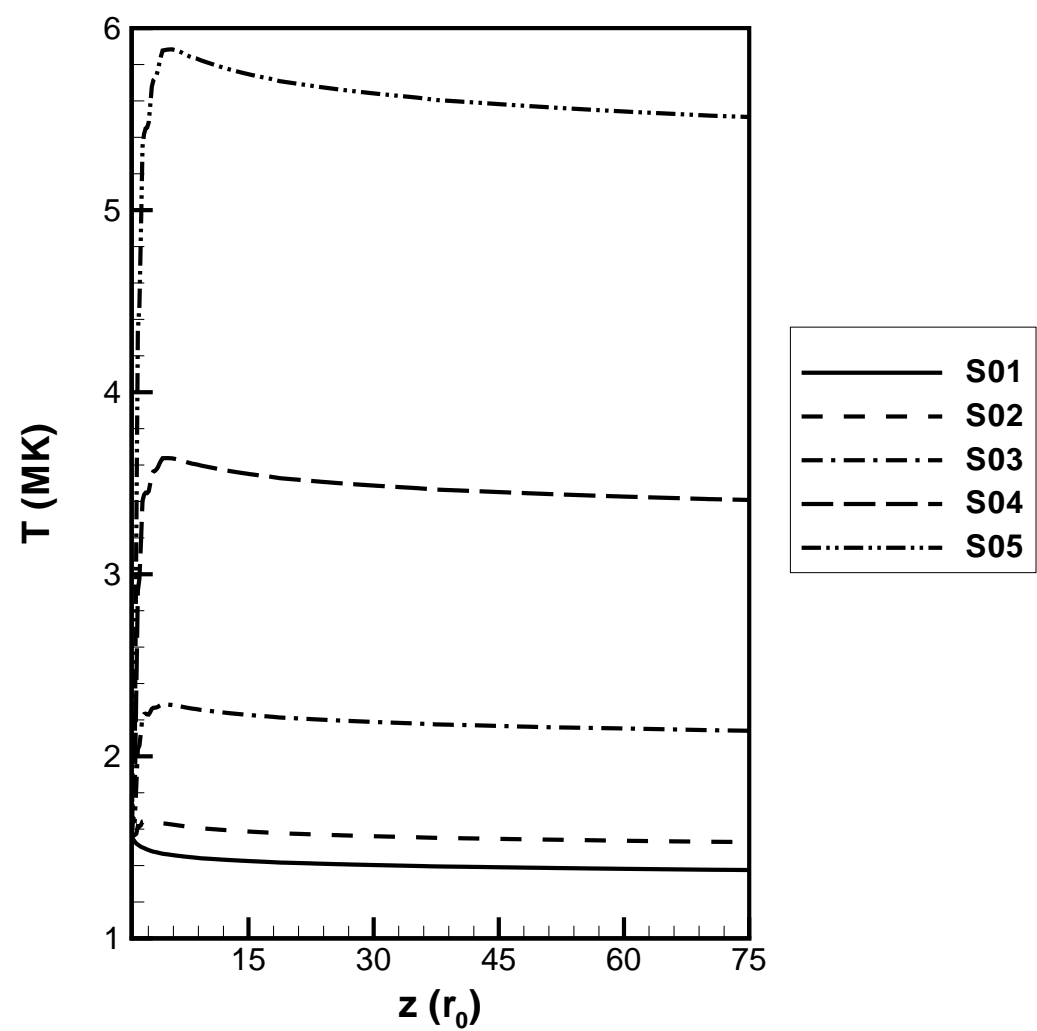

Figure 4.8: Temperature profile along the polar axis. 
where $\mathbf{q}$ is the total energy flux given by

$$
\mathbf{q}=\mathbf{u}\left(\frac{\rho u^{2}}{2}+\frac{\gamma}{\gamma-1} p+\rho \psi\right)+\mathbf{S}
$$

The terms on the RHS of Eq. (4.9) are the kinetic, enthalpy, and gravitational energy fluxes, and $\mathbf{S}$ is the Poynting flux vector that, in ideal MHD, is given by

$$
\mathbf{S}=\frac{B^{2}}{4 \pi} \mathbf{u}-\frac{(\mathbf{u} \cdot \mathbf{B})}{4 \pi} \mathbf{B}
$$

Taking a volume defined by a given magnetic flux tube bounded by two cross-sections (see Fig. 4.9), the net energy flux q should be conserved. This is verified in our simulations. Table 4.5 presents each parcel of the total energy flux that crosses four crosssectional areas of a magnetic flux tube whose central axis is located at $\theta \sim 30^{\circ}$. At $2.2 r_{0}$, the enthalpy power (i.e., the thermal plus internal energy fluxes integrated over the area of the cross-section of the tube at this position) is already the dominant form of energy. At $3.0 r_{0}$, magnetic power is almost negligible indicating that the continuous acceleration of the flow from there on is driven by solely the enthalpy. At $r=4.2 r_{0}$ and $6.0 r_{0}$, the conversion of enthalpy into kinetic energy is responsible for the continuous acceleration of the wind until a terminal velocity is reached.

Table 4.5: Percentage amount of energy flux passing through four different cross-sections at $2.2 r_{0}, 3 r_{0}, 4.2 r_{0}$, and $6 r_{0}$, along a flux tube for S03 and S05.

\begin{tabular}{lrrrr}
\hline \hline Energy Flux & $2.2 r_{0}$ & $3 r_{0}$ & $4.2 r_{0}$ & $6 r_{0}$ \\
\hline \multicolumn{5}{c}{$\mathrm{S} 03$} \\
Magnetic (\%) & 0.15 & 0.07 & 0.02 & 0.01 \\
Kinetic (\%) & 0.32 & 0.59 & 1.26 & 1.76 \\
Enthalpy (\%) & 97.08 & 97.63 & 97.50 & 97.39 \\
Gravitational (\%) & 2.45 & 1.72 & 1.22 & 0.85 \\
\hline & $\mathrm{S} 05$ & & & \\
\hline Magnetic (\%) & 0.41 & 0.15 & 0.02 & 0.02 \\
Kinetic (\%) & 0.46 & 0.85 & 1.77 & 2.33 \\
Enthalpy (\%) & 98.04 & 98.27 & 97.72 & 97.31 \\
Gravitational (\%) & 1.09 & 0.72 & 0.50 & 0.34 \\
\hline
\end{tabular}




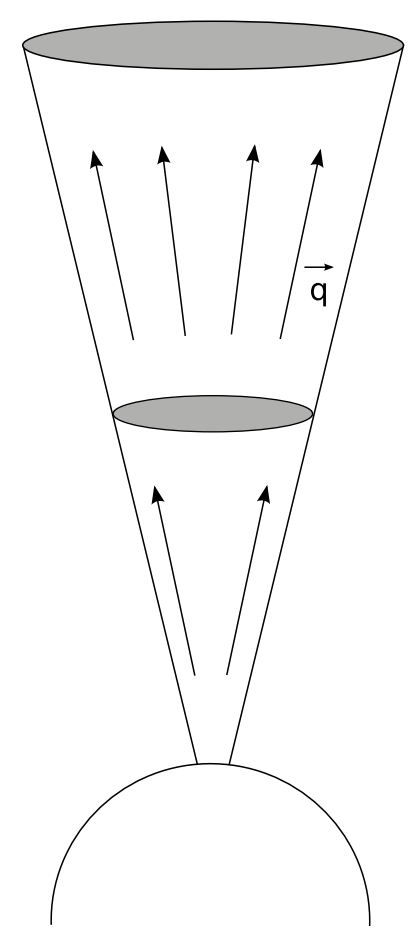

Figure 4.9: Cartoon of the energy flux $\mathbf{q}$ along a flux tube.

\subsection{The Second Set of Simulations}

The second set of simulations aims at investigating the effect of the density on the wind. We vary the initial density maintaining the same surface magnetic field $B_{0}$ and surface temperature $T_{0}$ adopted in S05. The simulations we analyze in this section consist of simulations S06 to S09 (Table 4.1).

We note that as $\rho_{0}$ increases, there is a decrease in the wind velocity, as can be seen in Fig. 4.10, where we plotted the radial velocity of the flows along $\theta=45^{\circ}$. Also, as $\rho_{0}$ is increased, the reconnection point moves closer to the star.

\subsubsection{Comparison Between First and Second Sets}

Comparing the first and the second set of simulations, we note that flows with same $\beta_{0}$ possess the same wind velocity profiles (S01/S09, S02/S08, S03/S07, and S04/S06). This result can be verified from Eqs. (3.2) to (3.5). The magnetic field can be written in terms of a normalized field $\tilde{B}$ with a normalization constant $B_{0}$

$$
\mathbf{B}=\tilde{\mathbf{B}} B_{0}
$$




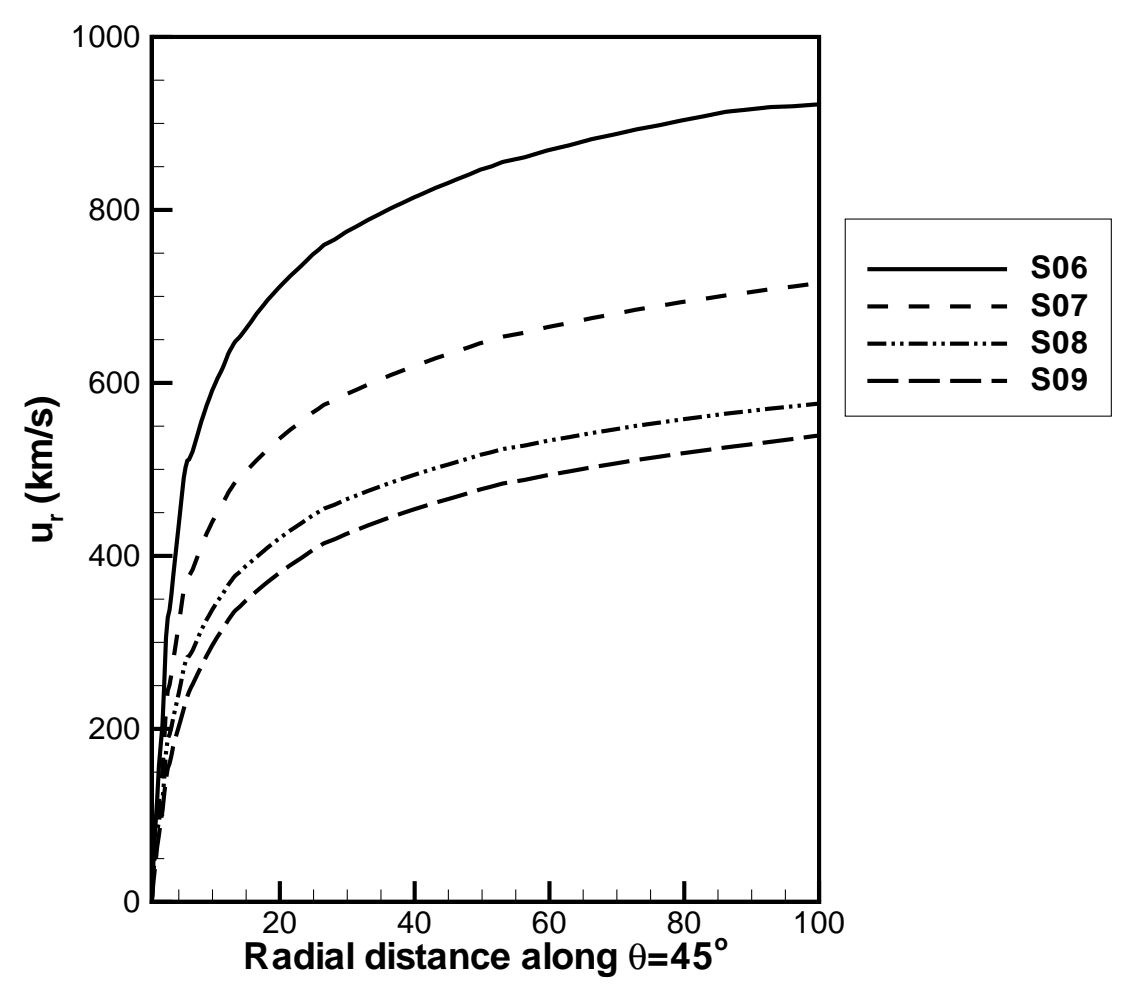

Figure 4.10: Radial velocity profiles as a function of the distance along $\theta=45^{\circ}$ for the second set of simulations.

Doing the same for the density

$$
\rho=\tilde{\rho} \rho_{0}
$$

In this case, Eqs. (3.2) to (3.5) are written as

$$
\begin{aligned}
& \frac{\partial \tilde{\rho}}{\partial t}+\nabla \cdot(\tilde{\rho} \mathbf{u})=0 \\
& \frac{\partial(\tilde{\rho} \mathbf{u})}{\partial t}+\nabla \cdot\left[\tilde{\rho} \mathbf{u} \mathbf{u}+\left(\tilde{p}+\frac{B_{0}^{2}}{\rho_{0}} \frac{\tilde{B}^{2}}{8 \pi}\right) I-\frac{B_{0}^{2}}{\rho_{0}} \frac{\tilde{\mathbf{B}}}{4 \pi}\right]=\tilde{\rho} \mathbf{g} \\
& \frac{\partial \tilde{\mathbf{B}}}{\partial t}+\nabla \cdot(\mathbf{u} \mathbf{\mathbf { B }}-\tilde{\mathbf{B}} \mathbf{u})=0 \\
& \frac{\partial \tilde{\varepsilon}}{\partial t}+\nabla \cdot\left[\mathbf{u}\left(\tilde{\varepsilon}+\tilde{p}+\frac{B_{0}^{2}}{\rho_{0}} \frac{\tilde{B}^{2}}{8 \pi}\right)-\frac{B_{0}^{2}}{\rho_{0}} \frac{(\mathbf{u} \cdot \tilde{\mathbf{B}}) \tilde{\mathbf{B}}}{4 \pi}\right]=\tilde{\rho} \mathbf{g} \cdot \mathbf{u},
\end{aligned}
$$

where

$$
\tilde{\varepsilon}=\frac{\tilde{\rho} u^{2}}{2}+\frac{\tilde{p}}{\gamma-1}+\frac{B_{0}^{2}}{\rho_{0}} \frac{\tilde{B}^{2}}{8 \pi} .
$$


Equations (4.11) and (4.13) show that the normalized density profile $(\tilde{\rho})$ and the normalized field profile $(\tilde{B})$ are independent of the values $\rho_{0}$ and $B_{0}$ adopted at the surface. Equations (4.12) and (4.14) show that the choice of $\rho_{0}$ and $B_{0}$ (or in other words the choice of $\beta_{0} \propto \rho_{0} / B_{0}^{2}$, as we assumed the same base temperature) appears explicitly in the momentum and energy equations. According to Eqs. (4.11) to (4.14), it is expected that flows with same $\beta_{0}$ have the same wind velocity, magnetic field configuration, density profile, etc. Although the normalized density and magnetic field profiles are the same if $\beta_{0}$ is the same in two different simulations, the mass-loss rates are different, where the value of the normalization constant $\rho_{0}$ has to be considered.

Hence, by analyzing only the velocity of an outflow, one cannot equivocally predict its physical characteristics at its base. Our results indicate that there is a group of magnetized flows that would present the same terminal velocity despite of its thermal and magnetic energy densities, as long as $\beta_{0}$ is the same.

This degeneracy can be removed if we consider the mass-loss rate. As the velocity profiles for two different magnetized outflows with similar $\beta_{0}$ are the same, the difference between the mass-loss rates of these winds comes from different density structures. To illustrate, consider cases S01 and S09 with $\beta_{0}=1$ : both cases present the same velocity profile and magnetic field configuration (although the intensity is not equal); but the density, and consequently the mass-loss rate, of S09 is 400 times larger than S01 in the entire grid.

\subsection{The Third Set of Simulations with $\gamma=1.1$}

Throughout Chap. 4, we have assumed that $\gamma=1.01$. We investigate now how the wind structure will change if a different $\gamma$ is considered. Considering the cases S01/S09 and $\mathrm{S} 02 / \mathrm{S} 08$, where $\beta_{0}=1,1 / 25$, respectively, we performed simulations considering $\gamma=1.1$. This third set of simulations is presented in Table 4.1 and the meridional cuts of the steady-state configurations for S01b and S02b are shown in Fig. 4.11.

It is worth noting that by adopting a different $\gamma$, the acceleration mechanism of the wind changes, what changes the velocity profile of the wind and, consequently, the configuration of the magnetic field (compare, for instance, the steady-state configuration of S01 and S01b). This is expected because, the higher $\gamma$ is, the thermal acceleration 

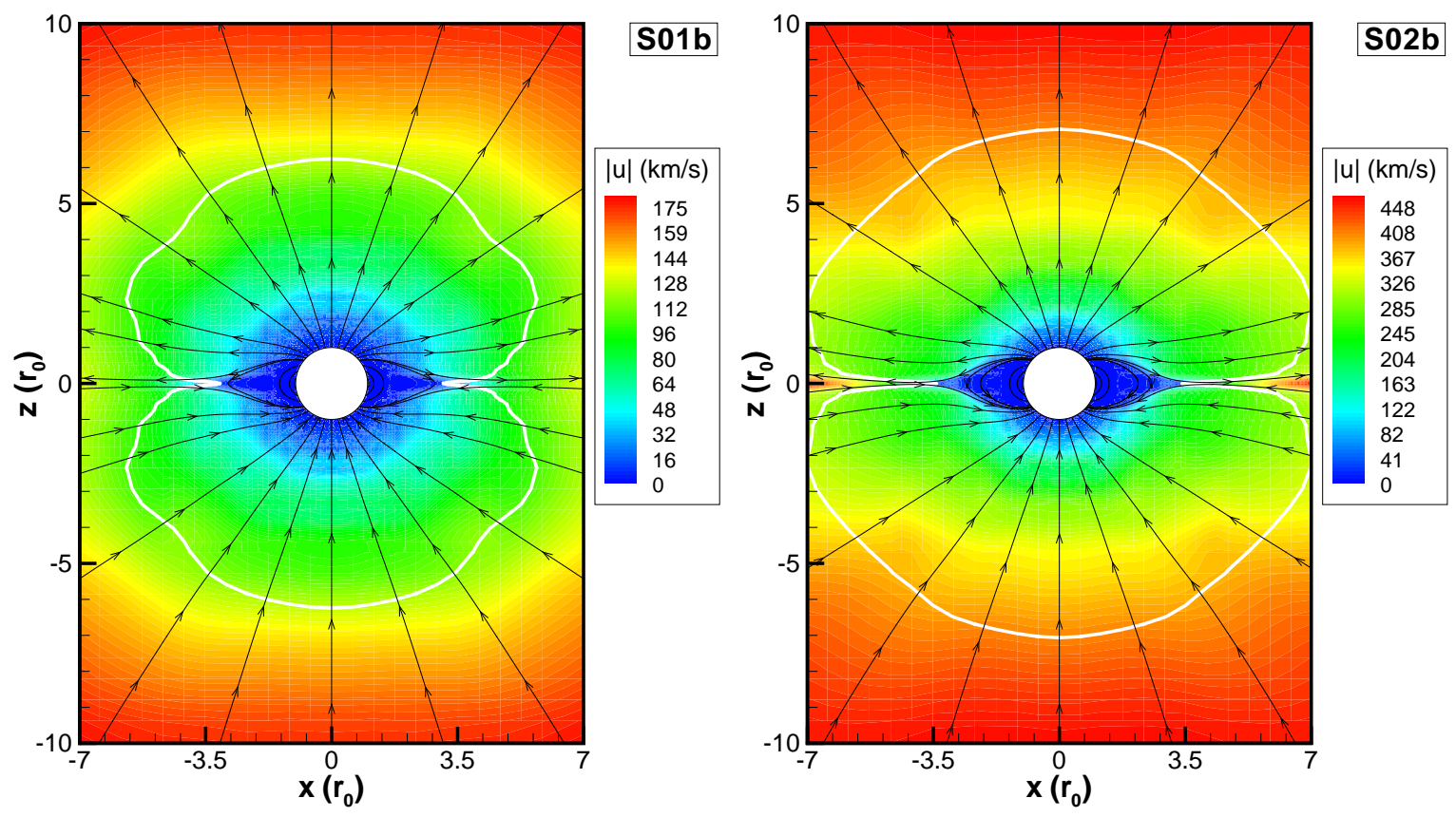

Figure 4.11: Same as Fig. 4.3, but for simulations S01b and S02b, where $\gamma=1.1$.

mechanism is less effective (e.g., there is less turbulence or dissipation of waves).

Due to the scaling relations described in $\S 4.2 .1$, simulations S01b and S09b, with $\beta_{0}=1$, present the same configuration of the steady-state wind. The same result is achieved for simulations S02b and S08b, with $\beta_{0}=1 / 25$.

Although $\gamma$ is necessary for the thermal acceleration of the wind, a wind with a high $\gamma$ implies a proportionally high Lorentz force, when compared to the thermal force. For this reason, the third set of simulations is more asymmetrical than the first set (compare S01 and S01b, S02 and S02b), since the Lorentz force is $\theta$-dependent. Another aspect is that in a purely HD coronal wind, the thermal force is the main responsible for the acceleration of the wind. This implies that by adopting a high $\gamma$, the wind has less internal energy available, being less thermally accelerated. In the cases S01 $(\gamma=1.01)$ and S01b $(\gamma=1.1)$, the same situation holds: case S01b is less accelerated than case S01. However, cases $\mathrm{S} 02(\gamma=1.01)$ and $\mathrm{S} 02 \mathrm{~b}(\gamma=1.1)$ show a different behavior. The present simulations indicate that a change in $\gamma$ from 1.01 to 1.1 decreases the terminal speed of the wind by $43 \%$ in cases S01/S01b, but increases the terminal speed by $8 \%$ in cases $\mathrm{S} 02 / \mathrm{S} 02 \mathrm{~b}$. This different behavior is because in case S02b the Lorentz force provides a significant acceleration mechanism for the wind, i.e., the Lorentz force is proportionally 
more important than the thermal force. This illustrates the need to perform full 3D MHD simulations in order to assess the importance of $\beta$ and $\gamma$.

\subsection{Discussion and Conclusion}

This is the first study to perform 3D MHD numerical simulations analyzing in detail winds of magnetized solar-like stars with different $\beta_{0}$. As initial condition, our model assumes a bipolar configuration for the magnetic field at the stellar surface. This distribution of magnetic field, although very simplified, is useful as a first step in understanding how the interaction between stellar magnetic field and the wind occurs in a 3D self-consistent manner.

In our simulations, we adopt a simpler treatment and parameterize the energy content of the wind in terms of $\gamma$. This is a first step towards more realistic simulations. It should be stressed that if one aims at describing the complex physics of stellar winds, a more complete description of the energy content of the wind has to be taken into account. As the goal of our study is to investigate the effects of the magnetic fields in the general properties of the stellar wind, the inclusion of MHD waves or variable $\gamma$ is postponed for future work (see Chap. 5).

In the first set of simulations where a fixed heating parameter $\gamma=1.01$ was adopted, we showed that for a solar-like star, the increase in the magnetic field intensity creates faster winds, with general characteristics as the Pneuman and Kopp (1971) result for the Sun: a creation of a bimodal stellar wind. The final configuration of the magnetic field consists of a zone of closed magnetic loops at low latitudes. On top of the closed loop region lies the neutral point, where the reconnection takes place and is the starting point of the current sheet that extends for larger distances along the equatorial plane. The zone of open field lines located at high latitudes of the star fills all the space outside the closed loop region and carries along a wind.

For a given $\beta_{0}$, the wind is more accelerated in the polar regions than in the equatorial region. This difference is generated by the latitude-dependent Lorentz force which creates a flux of matter directed to the equatorial plane. This generates a density enhancement at low latitudes providing a mass-loss rate per unit solid angle that is also latitudedependent and increases at low latitudes. This meridional flux is intensified for higher 
magnetic fields. The increase in the magnetic field also led to hotter winds with higher mass-loss rates.

We showed that the $\beta$-parameter is a key parameter in the steady-state structure of the wind in case $\gamma$ is the same for all simulations. As $\beta_{0}$ was increased back to 1 by changing $\rho_{0}$ and maintaining $B_{0}=20 \mathrm{G}$ in the second set of simulations, the wind decelerated and cooled. Comparing the first and second sets of simulations, we conclude that winds with same $\beta_{0}$ have (i) same velocity profile and (ii) same magnetic field topology (the neutral point is located at the same position). However, mass-loss rates are different as $\dot{m}(\theta)$ is dependent on the choice of $\rho_{0}$.

By analyzing the effects of a different $\gamma$ in the simulations, we showed in the third set of simulations that the heating parameter $\gamma$ is, together with the plasma- $\beta$ parameter at the coronal base, an important parameter in the structure of the wind. However, the steady-state configuration of the wind in the third set is different from the first and second ones because the thermal force driving the wind changes when $\gamma$ changes.

The effective temperature of solar-like stars is orders of magnitude lower than the coronal temperature adopted in our models. In the present work, we did not take into account what is heating the wind from photospheric temperatures up to coronal temperatures. Instead, we placed the inner boundary of our model at the coronal base. For a more realistic study, however, the energy equation should be solved starting from lower layers. Hence, the plasma- $\beta$ parameter at the coronal base would then be incorporated as resultant from the energy interactions happening in these lower layers rather than being a free parameter at the coronal base, as in our models. Models and 1D simulations that resolve the energy balance in the chromosphere-corona transition region have been calculated recently for the solar wind (Suzuki and Inutsuka 2005, Cranmer et al. 2007) and stellar winds (Suzuki 2007, Cranmer 2008). If incorporated in 3D global simulations, these models can provide physical insights on the processes that accelerate and heat stellar winds.

The present work considered moderate $\beta_{0}$. In the presence of a weak magnetic field (high $\beta_{0}$ ), the wind is energetic enough to drag the field lines with it, leading to a configuration of radial magnetic field lines. Consequently, a spherical expansion of the corona is expected, similar to a purely HD case. 


\section{Chapter 5}

\section{Energetic Processes in Coronal Winds}

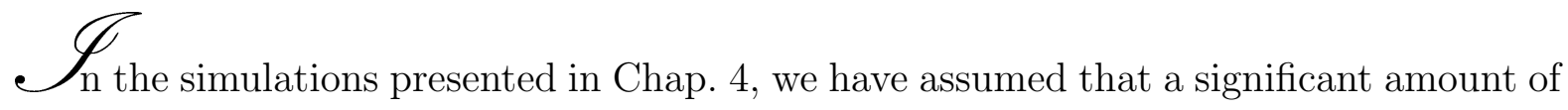
energy is stored in the internal degrees of freedom of the plasma. In practice, this means that $\gamma$, the ratio of the specific heats of the gas, is not, e.g., the usual 5/3-value used for monoatomic species, but a value that simulates internal energy addition to the particles of the wind. In Chap. 4, we have adopted $\gamma=1.01$ and 1.1. Furthermore, observations of the solar corona have shown that $\gamma$ is not constant along the solar wind. It has been inferred that closer to the Sun, $\gamma \simeq 1$ (Steinolfson and Hundhausen 1988), and at $1 \mathrm{AU}$, $\gamma \simeq 1.5$ (Totten et al. 1995, Phillips et al. 1995). In this chapter, we develop a way to mimic a more realistic energetic input in the wind by letting $\gamma$ vary and compare to what has been presented earlier on this thesis.

\subsection{Mimicking Energetic Processes}

Due to the lack of knowledge of all the detailed processes that take place in a stellar wind, it is difficult to estimate all the mechanisms that modify the heat content of the wind. Conduction, radiation, mechanical dissipation of energy that is transferred to the plasma, among others, all contribute to the heating and cooling of the wind. MHD waves and turbulence are known to play important roles on the solar wind acceleration and heating (Matthaeus et al. 1999, Cranmer and van Ballegooijen 2005, Cranmer et al. 2007) and, 
for this reason, they are often included in stellar wind models as well (e.g., Airapetian et al. 2000, Vidotto and Jatenco-Pereira 2006; 2009, Vidotto et al. 2006, Suzuki 2007). The connection between the solar wind and the stellar winds is usually made by scaling the observed solar wind quantities (such as, the wave flux and its spectral slope, surface magnetograms, etc.) to other stars (Jatenco-Pereira and Opher 1989, Schrijver 2001). This is a valuable method, however it is not clear how these observables scale to other solar-like stars.

In Chap. 4, we used a simpler treatment of the mechanisms of acceleration of the wind [similarly to Roussev et al. (2003)] by considering that $\gamma$ is associated with "turbulent" internal degrees of freedom, in a way analogous to the Sun, where a significant amount of energy is stored in the form of waves and turbulent fluctuations. In this treatment, the value of $\gamma$ accounts for the additional heating stored as the internal energy.

To avoid confusion, let us call $\Gamma$ as the usual ratio of the specific heats (e.g., $\Gamma=5 / 3$ for monoatomic species), as derived from thermodynamics, and $\gamma$ the heating index that we used in Chap. 4. The energy equation [Eq. (3.5)] is rewritten as

$$
\frac{\partial}{\partial t}\left(\frac{\rho u^{2}}{2}+\frac{p}{\gamma-1}+\frac{B^{2}}{8 \pi}\right)+\nabla \cdot\left[\mathbf{u}\left(\frac{\rho u^{2}}{2}+\frac{\gamma}{\gamma-1} p+\frac{B^{2}}{4 \pi}\right)-\frac{(\mathbf{u} \cdot \mathbf{B})}{4 \pi} \mathbf{B}\right]=\rho \mathbf{g} \cdot \mathbf{u} .
$$

In terms of a constant $\Gamma$, the last equation is written as

$$
\frac{\partial}{\partial t}\left(\frac{\rho u^{2}}{2}+\frac{p}{\Gamma-1}+\frac{B^{2}}{8 \pi}\right)+\nabla \cdot\left[\mathbf{u}\left(\frac{\rho u^{2}}{2}+\frac{\Gamma}{\Gamma-1} p+\frac{B^{2}}{4 \pi}\right)-\frac{(\mathbf{u} \cdot \mathbf{B})}{4 \pi} \mathbf{B}\right]=\rho \mathbf{g} \cdot \mathbf{u}+q,
$$

where $q$ is the energy input. Combining the two last equations, we can find the amount of energy that is input in the wind when we consider the heating index $\gamma$ instead of the ratio of specific heats $\Gamma=5 / 3$

$$
q=\frac{\partial}{\partial t}\left(\frac{p}{\Gamma-1}-\frac{p}{\gamma-1}\right)+\nabla \cdot\left[\mathbf{u}\left(\frac{1}{\Gamma-1}-\frac{1}{\gamma-1}\right) p\right] .
$$

\subsubsection{Derivation of the Energy Equation}

Equations (5.1) to (5.3) are valid as long as $\gamma$ and $\Gamma$ are constants in space and time. A way to mimic energy deposition in the wind, and therefore, increase the internal energy of the particles, was suggested by Roussev et al. (2003) and later on also adopted by Cohen et al. (2007), in the study of the solar wind. In such approach, it is assumed that $\gamma$ can vary with the distance to the star. Motivated by this method, we now aim 
at deriving a more general energy equation, similar to Eq. (5.1), but that allows for a variable $\gamma$. Note that more realistic magnetized wind models compute the heating of the wind caused due to physical processes, as is the case of the 1D models done by Vidotto and Jatenco-Pereira (2006; 2009), Vidotto et al. (2006), Falceta-Gonçalves et al. (2006), Cranmer et al. (2007), among others. However, inclusion of such processes, e.g. dissipation of waves or turbulence, in a $3 \mathrm{D}$ code is very challenging and is deferred for future studies.

We begin such derivation from the conservation of entropy, that in terms of the total derivative is given by

$$
\frac{\mathrm{D}}{\mathrm{D} t}\left(p \rho^{-\gamma_{v}}\right)=0
$$

The index $v$ is used as to differentiate $\gamma_{v}$, which is variable in time and space, from the constant $\gamma$. Hence

$$
p \frac{\mathrm{D} \rho^{-\gamma_{v}}}{\mathrm{D} t}+\rho^{-\gamma_{v}} \frac{\mathrm{D} p}{\mathrm{D} t}=0 .
$$

The first term in Eq. (5.5) is written as

$$
p \frac{\mathrm{D} \rho^{-\gamma_{v}}}{\mathrm{D} t}=p \rho^{-\gamma_{v}}\left(\gamma_{v} \nabla \cdot \mathbf{u}-\ln \rho \frac{\mathrm{D} \gamma_{v}}{\mathrm{D} t}\right)
$$

where we used Eq. (2.15). Equation (5.5) becomes

$$
\frac{\mathrm{D} p}{\mathrm{D} t}+p \gamma_{v} \nabla \cdot \mathbf{u}=p \ln \rho \frac{\mathrm{D} \gamma_{v}}{\mathrm{D} t}
$$

Expanding the term $\mathrm{D} p / \mathrm{D} t$ by using Eq. (2.16) and rewriting $p \gamma_{v} \nabla \cdot \mathbf{u}=\gamma_{v} \nabla \cdot(p \mathbf{u})-$ $\gamma_{v}(\mathbf{u} \cdot \nabla) p$, the previous equation becomes

$$
\begin{aligned}
(\mathbf{u} \cdot \nabla) p= & \frac{1}{\gamma_{v}-1} \frac{\partial p}{\partial t}+\frac{\gamma_{v}}{\gamma_{v}-1} \nabla \cdot(p \mathbf{u})-\frac{p}{\gamma_{v}-1} \ln \rho \frac{\mathrm{D} \gamma_{v}}{\mathrm{D} t} \\
(\mathbf{u} \cdot \nabla) p= & \frac{\partial}{\partial t}\left(\frac{p}{\gamma_{v}-1}\right)+\nabla \cdot\left(\frac{\gamma_{v}}{\gamma_{v}-1} p \mathbf{u}\right) \\
& +\frac{p}{\left(\gamma_{v}-1\right)^{2}}\left[1-\left(\gamma_{v}-1\right) \ln \rho\right] \frac{\mathrm{D} \gamma_{v}}{\mathrm{D} t}
\end{aligned}
$$

Taking the dot product of the equation of motion [Eq. (2.49)] with $\mathbf{u}$, we have

$$
\rho \mathbf{u} \cdot \frac{\mathrm{D} \mathbf{u}}{\mathrm{D} t}=-(\mathbf{u} \cdot \nabla) p+\rho \mathbf{u} \cdot \mathbf{g}+\mathbf{u} \cdot \frac{\mathbf{J} \times \mathbf{B}}{c} .
$$


Now, we include Eq. (5.9) in (5.10) and after some analytical work, the resultant equation gives rise to a modified energy equation. We do not need to follow each step of this derivation. By a careful look at Eq. (5.9), it is immediate to recognize the new extra terms that arose due to the non-constancy of $\gamma$, namely

$$
S\left(\gamma_{v}\right)=-\frac{p}{\left(\gamma_{v}-1\right)^{2}}\left[1-\left(\gamma_{v}-1\right) \ln \rho\right] \frac{\mathrm{D} \gamma_{v}}{\mathrm{D} t}
$$

Thus, the energy equation, modified by a variable $\gamma$, becomes

$$
\begin{array}{r}
\frac{\partial}{\partial t}\left(\frac{\rho u^{2}}{2}+\frac{p}{\gamma_{v}-1}+\frac{B^{2}}{8 \pi}\right)+\nabla \cdot\left[\mathbf{u}\left(\frac{\rho u^{2}}{2}+\frac{\gamma_{v}}{\gamma_{v}-1} p+\frac{B^{2}}{4 \pi}\right)-\frac{(\mathbf{u} \cdot \mathbf{B})}{4 \pi} \mathbf{B}\right] \\
=\rho \mathbf{g} \cdot \mathbf{u}+S\left(\gamma_{v}\right) .
\end{array}
$$

In the case $\gamma$ is constant $\left(\gamma=\gamma_{v}\right), S\left(\gamma_{v}\right)=0$ and we recover Eq. (5.1).

In the following results, we assume that $\gamma_{v}$ does not vary with time, and that it only depends on the radial coordinate. Hence, Eq. (5.11) becomes

$$
S\left(\gamma_{v}\right)=-\frac{p}{\left(\gamma_{v}-1\right)^{2}}\left[1-\left(\gamma_{v}-1\right) \ln \rho\right] u_{r} \frac{\partial \gamma_{v}}{\partial r} .
$$

In practice, this means that we are mimicking energy deposition in the wind that depends on the radial position, but not on time.

\subsubsection{Numerical Model}

Because the approach we have just derived requires a modification in the energy equation, BATS-R-US need to be modified so it can allow for the variable $\gamma$. The easiest way to modify BATS-R-US is to continue to solve Eqs. (3.2) to (3.5) as they are, and add a new equation (and thus a new variable) to this system of equations. To be consistent with the code, this new equation should be written in the conservative form [Eq. (3.1)].

We thus introduced a new variable $\varepsilon_{w}$, where the subscript " $w$ " indicates that this extra energy is stored in form of waves. Hence, the equation that describes the extra energy input due to $\gamma_{v}$ is

$$
\frac{\partial \varepsilon_{w}}{\partial t}+\nabla \cdot\left(\mathbf{u} \varepsilon_{w}\right)=S\left(\gamma_{v}\right)
$$

where

$$
\varepsilon_{w}=p\left(\frac{1}{\gamma_{v}-1}-\frac{1}{\gamma-1}\right) .
$$


It is easy to show that the sum of Eqs. (5.1) and (5.14) gives rise to the recently derived Eq. (5.12). In the limiting case of $\gamma_{v}=\gamma$ (i.e., constant $\gamma$ ), Eq. (5.14) vanishes.

The main goal of this chapter is to compare the results we achieved in Chap. 4, when a constant $\gamma=1.01$ was used, to the cases we present next, when a variable $\gamma$ is used. For this reason, we adopt the same parameters as in Chap. 4, namely that the star has $M_{\star}=1 M_{\odot}$ and $r_{0}=1 R_{\odot}$, the wind consists of a totally ionized plasma of hydrogen (i.e., $\mu=0.5$ ) and has a coronal base temperature of $T_{0}=1.56 \times 10^{6} \mathrm{~K}$. The adopted grid was described in $\S 3.2 .1$, with the initial ${ }^{1}$ and boundary conditions presented in $\S 3.2 .2$ and $\S 3.2 .3$, respectively.

Motivated by observations of the solar wind that $\gamma$ varies with the distance (Steinolfson and Hundhausen 1988, Totten et al. 1995, Phillips et al. 1995), we adopt a linear function that empirically describes the variation of $\gamma$

$$
\gamma_{v}=1.01+\frac{0.49}{214}\left(r-r_{0}\right)
$$

so that $\gamma_{v}\left(r_{0}\right) \simeq 1$ and $\gamma_{v}\left(215 r_{0}\right)=1.5$. One can understand the relation above as a more intense energy deposition near the star and, at $1 \mathrm{AU}\left(\simeq 215 r_{0}\right)$, the energy input is less dramatic (the case where $\gamma=\Gamma=5 / 3 \simeq 1.66$ means adiabatic expansion, i.e., there is neither input nor loss of energy). The dashed-line in Fig. 5.1a represents Eq. (5.16), while the solid line represents the constant value of $\gamma=1.01$, as used in Chap. 4. Figure 5.1b presents the initial radial velocity profiles for both cases. As a consequence of the larger energetic input in the case where $\gamma$ is kept constant throughout the wind, we note that the wind is more accelerated than in the case where $\gamma$ is varying accordingly to Eq. (5.16).

\subsection{Results}

In this chapter, we perform two simulations whose parameters used are presented in Table 5.1. As it can be seen, we are adopting the same parameters as S01 and S02 (Chap. 4), except for $\gamma$. The reason for that is because we would like to compare the results from the two different approaches for energy input in the simulations: constant versus variable $\gamma$.

\footnotetext{
${ }^{1}$ In the simulations shown in this chapter, the initial values of velocity and density profiles have to take into account that $\gamma$ is not constant.
} 

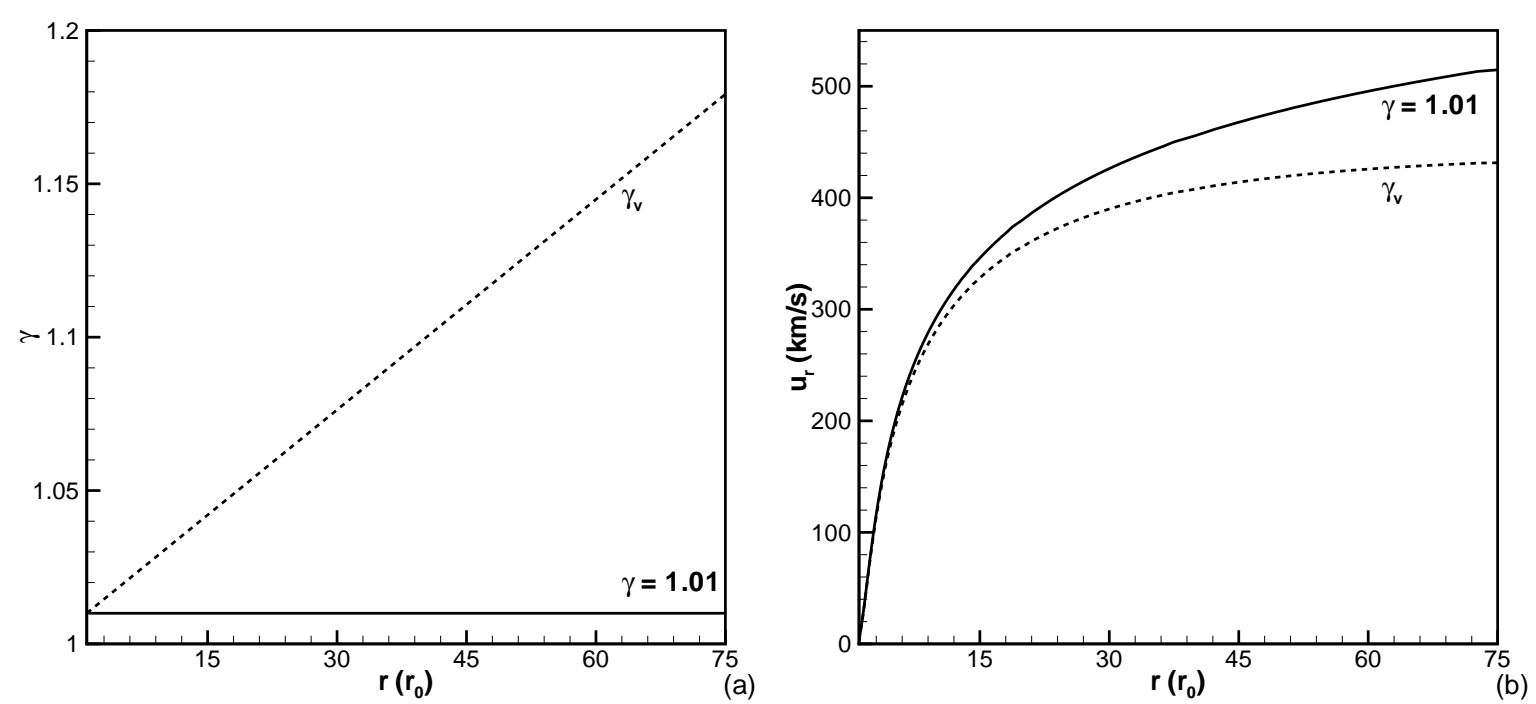

Figure 5.1: (a) Radial dependence of the $\gamma$ adopted. (b) Initial radial velocity profiles. Solid line: constant $\gamma(\gamma=1.01)$; dashed line: variable $\gamma[E q$. (5.16)].

Figure 5.2 shows the meridional cuts of total velocity for V01, V02, S01, and S02, when simulations have achieved steady state. We also present streamlines of magnetic field (black lines), together with the Alfvén surface (white line). We note that simulations S01 and V01 are very similar to each other: they present similar velocity profiles, magnetic configuration, and also Alfvén surface. A careful analysis shows subtle differences though. For example, the acceleration of S01 occurs nearer from the central star than for V01, thus S01 presents slightly larger velocities than V01. This results in a small difference in the density profile, with V01 having larger densities and larger mass-loss rates. We also note that the volume of the closed line region is larger for V01. Simulations S02 and V02 also present similar steady-state results with subtle differences: the mass-loss rate, density profiles, and velocity profiles of V02 are larger than of S02; V02 also presents a

Table 5.1: The set of simulations performed with a variable $\gamma[E q .(5.16)]$. The columns represent, respectively: the name of the simulation, the temperature and density at the coronal base, the magnetic field intensity at the pole, and the value of $\beta$ evaluated at the pole.

\begin{tabular}{|c|c|c|c|c|}
\hline $\begin{array}{c}\text { Name } \\
-\end{array}$ & $\begin{array}{c}T_{0} \\
\left(10^{6} \mathrm{~K}\right)\end{array}$ & $\begin{array}{c}\rho_{0} \\
\left(\mathrm{~g} \mathrm{~cm}^{-3}\right)\end{array}$ & $\begin{array}{l}B_{0} \\
(\mathrm{G})\end{array}$ & $\begin{array}{l}\beta_{0} \\
-\end{array}$ \\
\hline V01 & 1.56 & $1.544 \times 10^{-16}$ & 1 & 1 \\
\hline V02 & 1.56 & $1.544 \times 10^{-16}$ & 5 & $1 / 25$ \\
\hline
\end{tabular}



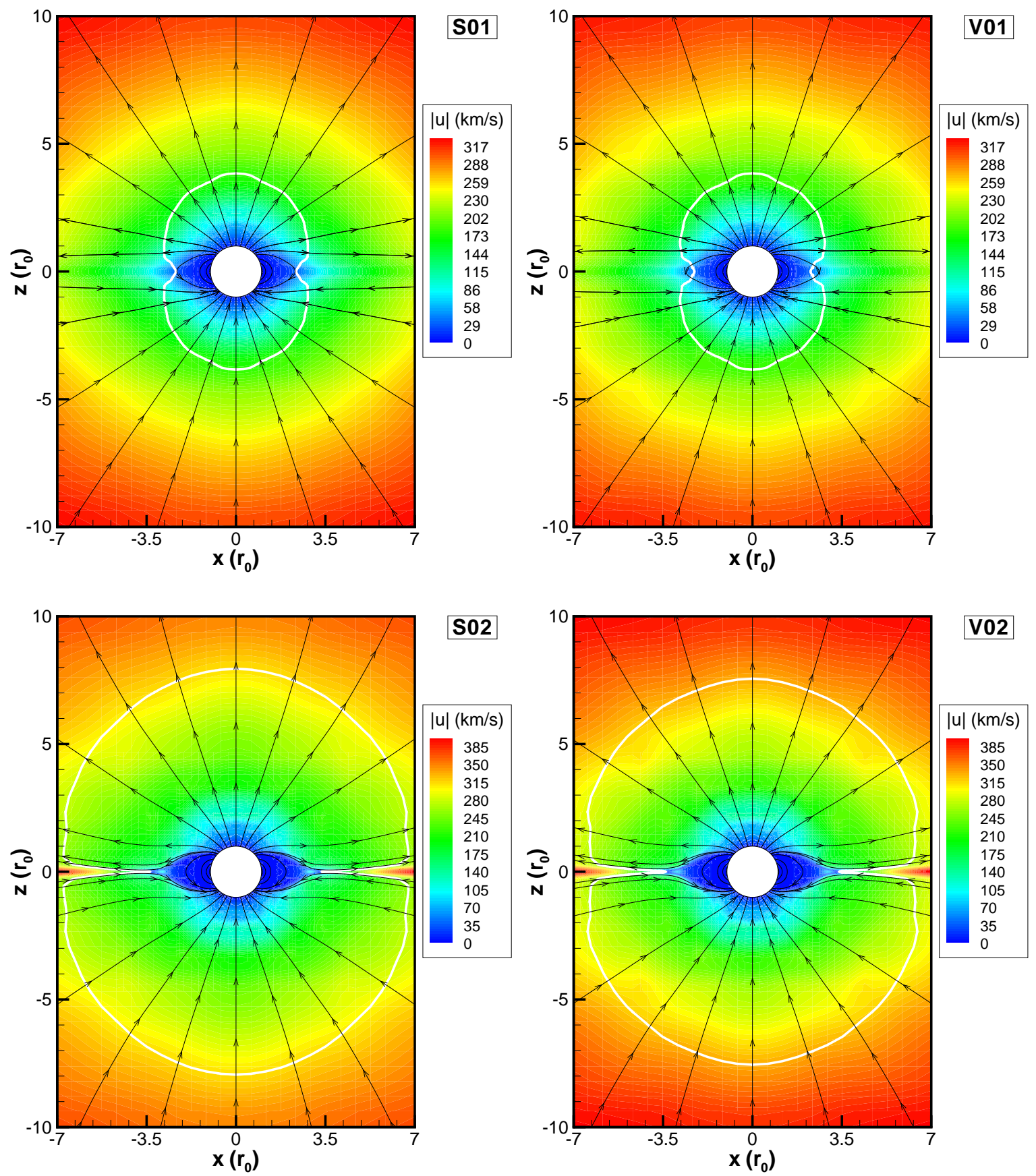

Figure 5.2: Meridional cuts of the steady-state configurations for simulations V01, V02, S01, and S02. Black streamlines represent the final configuration of the magnetic field. Contour plots of the velocity of the flow are shown in the background. The white line is the Alfvén surface.

larger region of closed lines compared to S02. 
These results show that the resultant wind structure using the approach of variable $\gamma$ in order to mimic energetic processes in the wind does not differ considerably from the steady-state wind structure derived from the approach with constant $\gamma$. It should be noted however that the relation expressed in Eq. (5.16) provides a very smooth transition between the region where $\gamma_{v} \simeq 1$ and the region where $\gamma_{v}=1.5$ (i.e., $\gamma_{v}$ varies slowly from the proximity of the star up to $1 \mathrm{AU})$. A steep transition would result in a more significant change in the wind structures derived from the two different approaches.

\subsubsection{Application: Estimates of X-ray Emission Measure}

The magnetically confined hot coronal plasma $\left(T \gtrsim 10^{6} \mathrm{~K}\right)$ of solar-like stars emits the bulk of its radiative energy in the X-ray range (Schmitt and Wichmann 2001). For this reason, the detection of soft X-ray radiation can diagnose coronal emission. In cool lowmass stars (either in the MS as well as in the PMS phases), the coronal plasmas are responsible for most of the X-ray emission detected. There are evidences that the X-ray luminosity $L_{X}$ increases with stellar mass (for T Tauri stars, $L_{X} \propto M_{\star}^{1.7 \pm 0.1}$, Telleschi et al. 2007) and with magnetic flux $\Phi$ (for the Sun, G, K, and M dwarfs, and T Tauri stars $L_{X} \propto \Phi^{1.13 \pm 0.05}$, Pevtsov et al. 2003).

In this section, we aim at estimating the optically thin X-ray luminosity from the coronae of solar-like stars. We calculate $L_{X}$ for simulations V01 and V02, and compare these results to S01 and S02, respectively. The power emitted in an optically thin emission line is proportional to the emission measure of the emitting matter

$$
E_{M}=\int_{V} n_{i} n_{e} \mathrm{~d} V
$$

where $n_{i}$ and $n_{e}$ are the number densities of ions and electrons, respectively, and $V$ is the volume of the region where the X-ray emission is generated. In the simulations we performed, we adopted $n_{e}=n_{i}=n$ for simplicity. If one assumes that the X-ray emission is optically thin, from the emission measure, the X-ray luminosity can be estimated as

$$
L_{X}=E_{M} \Lambda(\langle T\rangle)
$$

where $\Lambda(\langle T\rangle)$ is the radiative cooling function of the plasma, which here we assume to depend solely on the mean temperature $\langle T\rangle$ of the region. We use the values of $\Lambda$ computed by Schmutzler and Tscharnuter (1993). Assuming that the X-ray emission of 
Table 5.2: Emission measure and X-ray luminosity generated inside the region of closed field lines for V01, V02, S01, and S02. The columns represent, respectively: the name of the simulation, the radial dependence of $\gamma$, the volume $V$ defined by the closed field lines, the estimated emission measure, the mean temperature inside $V$, and the estimated X-ray luminosity.

\begin{tabular}{cccccc}
\hline \hline Name & $\gamma(r)$ & $V$ & $E_{M}$ & $\langle T\rangle$ & $L_{X}$ \\
- & - & $\left(r_{0}^{3}\right)$ & $\left(10^{48} \mathrm{~cm}^{-3}\right)$ & $\left(10^{6} \mathrm{~K}\right)$ & $\left(10^{26} L_{\odot}\right)$ \\
\hline V01 & variable & 34 & 8.44 & 1.54 & 11.1 \\
S01 & constant & 31 & 8.49 & 1.51 & 9.67 \\
\hline V02 & variable & 24 & 10.6 & 1.81 & 9.60 \\
S02 & constant & 23 & 10.8 & 1.66 & 9.35 \\
\hline
\end{tabular}

the coronae of solar-like stars comes from the closed field line region, we calculate the emission measure [Eq. (5.17)] and the X-ray luminosity [Eq. (5.18)] for V01, V02, S01, and S02, and present them in Table 5.2, along with the mean temperature of the confined plasma and the volume of the emitting region.

From Table 5.2, we note that the volume of the closed field line region is larger for the simulations where $\gamma$ is variable. Despite of that, the emission measure calculated for the simulations with variable $\gamma$ results in slightly smaller values than for the simulations with constant $\gamma$, a consequence of smaller densities in cases S01 (S02) with respect to V01 (V02), respectively. When we compare the X-ray luminosity, the opposite takes place: the X-ray luminosity calculated for the simulations where $\gamma$ is constant is smaller than for variable $\gamma$, due to slightly lower temperatures in S01 and S02.

The X-ray luminosities presented in Table 5.2 are of the order of $\sim 10^{27} \mathrm{erg} \mathrm{s}^{-1}$. It should be reminded here that we are performing estimates: we are not considering a real chemical composition of the corona, neither a two-component temperature plasma, which are usually taken into account in the derivation of the properties of the emitting region. Nevertheless, when compared to values from Pevtsov et al. (2003) for G, K, and $\mathrm{M}$ dwarfs $\left(10^{27}\right.$ to $\left.10^{29} \mathrm{erg} \mathrm{s}^{-1}\right)$, and to the Sun, which has $L_{X} \sim 10^{26}$ to $10^{27} \mathrm{erg} \mathrm{s}^{-1}$, we see that our results present roughly the observed $L_{X}$ for MS solar-like stars. 


\subsection{Final Remarks}

In this chapter, we have showed that the wind properties, especially the observable values of mass-loss rates, terminal velocities, and X-ray luminosities, calculated through the approach of a constant heating parameter $\gamma$ for the wind is very similar to the wind properties derived from the approach of a $\gamma$ that has a linear radial dependence with the distance to the central star [Eq. (5.16)]. Our main goal of this chapter was to validate the approach of constant $\gamma$ that was used in Chap. 4. In face of this similarity, in the remainder of this thesis, we will maintain the approach adopted in Chap. 4, which considers that $\gamma$ is constant throughout the wind.

As discussed in $\S 5.2$, a steeper variation of $\gamma$ may result in more significant changes in the wind structure. 


\section{Chapter 6}

\section{D Simulations of Winds of T Tauri Stars and The Influence of the Wind on Planet Migration}

e extend the model presented in Chap. 4 to the study of magnetized winds of PMS stars. We focus on weak-lined T Tauri stars (WTTSs), as the presence of the tenuous accretion disk is not expected to have strong influence on the structure of the stellar wind. We aim at investigating the coronal magnetic configuration of WTTSs using 3D MHD numerical simulations. T Tauri stars are believed to be magnetized, and rotating several times faster than the Sun, hence it is important to consider a magneto-centrifugal wind. The results presented here were published in the Astrophysical Journal, 2009, 703, 1734 (Vidotto et al. 2009b).

As discussed in Chap. 1, the structure of a magnetized stellar wind significantly depends on both the field intensity and topology. In Chap. 4 (Vidotto et al. 2009a), we performed an analysis of coronal winds in MS solar-like stars. We showed that a decisive parameter in the acceleration of the magnetized wind of a solar-like star is the plasma- $\beta$ at the coronal base. Using 3D MHD self-consistent simulations, we explored how the change in the magnetic and thermal energy densities at the base of the wind modifies the magnetic configuration of the corona and the velocity profile of the wind. Rotation was not considered in that work.

We now investigate the magnetic configuration of the winds of WTTSs with the 
Table 6.1: The set of simulations for WTTSs. The columns represent, respectively: the name of the simulation, the surface magnetic field intensity at the pole, the period of rotation of the star, $\gamma$, the density at $r_{0}$, and the plasma- $\beta$ evaluated at the pole.

\begin{tabular}{cccccc}
\hline \hline Name & $B_{0}$ & $P_{0}$ & $\gamma$ & $\rho_{0}$ & $\beta_{0}$ \\
- & $\left(10^{3} \mathrm{G}\right)$ & $(\mathrm{d})$ & - & $\left(\mathrm{g} \mathrm{cm}^{-3}\right)$ & - \\
\hline $\mathrm{A}$ & 1 & 3 & 1.2 & $1 \times 10^{-11}$ & $1 / 25$ \\
$\mathrm{~B}$ & 0.2 & 3 & 1.2 & $1 \times 10^{-11}$ & 1 \\
$\mathrm{C}$ & 1 & 3 & 1.1 & $1 \times 10^{-11}$ & $1 / 25$ \\
$\mathrm{D}$ & 1 & 10 & 1.2 & $1 \times 10^{-11}$ & $1 / 25$ \\
$\mathrm{E}$ & 1 & 3 & 1.2 & $2.4 \times 10^{-10}$ & 1 \\
$\mathrm{~F}$ & 1 & 3 & 1.2 & $2.4 \times 10^{-12}$ & $1 / 100$ \\
$\mathrm{G}$ & 1 & 0.5 & 1.2 & $2.4 \times 10^{-12}$ & $1 / 100$ \\
\hline
\end{tabular}

addition of stellar rotation. In our models we vary different physical parameters of the systems, such as stellar rotational period, and magnetic field intensity and gas density at the base of the wind. In particular, we investigate the occurrence of helmet streamers or slingshot prominences, as well as their shape and extension. We perform self-consistent time-dependent 3D numerical simulations, including centrifugal, magnetic, gravitational, and thermal forces. The full set of MHD equations is solved to obtain the steady-state solution for the large-scale magnetic configuration of a $\mathrm{T}$ Tauri star.

The parameters used in the simulations can be seen in Table 6.1, where we present the maximum surface magnetic field intensity $B_{0}$, the stellar period of rotation $P_{0}$, the ratio of specific heats $\gamma$, the density at the base of the corona $\rho_{0}$, and $\beta_{0}$, the surface value of the plasma- $\beta$ evaluated at the pole. We chose to simulate extreme values of the stellar rotational period, magnetic field intensity, and density, in order to probe the possible magnetic configurations for WTTSs. The range of densities we adopted for the base of the stellar wind is $2.4 \times 10^{-12}$ to $2.4 \times 10^{-10} \mathrm{~g} \mathrm{~cm}^{-3}$. This choice of density results in mass-loss rates ranging between $1 \times 10^{-9}$ and $8 \times 10^{-8} \mathrm{M}_{\odot} \mathrm{yr}^{-1}$.

We use the numerical model presented in Chap. 3. We now adopt a star with $M_{\star}=$ $0.8 M_{\odot}$ and $r_{0}=2 R_{\odot}$ to be consistent with a typical WTTS. The stellar wind consists of a totally ionized plasma of hydrogen (i.e., $\mu=0.5$ ) and has a coronal base temperature of $T_{0}=1 \times 10^{6} \mathrm{~K}$. Although there are observations of very high temperature plasmas inside closed magnetic structures, as evidenced by X-ray measurements (Getman et al. 
2005, Güdel et al. 2007), the outflowing stellar wind is believed to be much cooler, as estimates presented by Johns-Krull and Herczeg (2007) from UV spectra of the CTTS TW Hya, and by Gómez de Castro and Verdugo (2007) for the CTTS RY Tau.

We simulate three different stellar periods of rotation: 10,3 , and $0.5 \mathrm{~d}$. It is known that when the star is rotating with velocities near the critical velocity $\left[v_{\text {crit }}=\right.$ $\left.\left(G M_{\star} / r_{0}\right)^{1 / 2}\right]$, its surface is deformed, becoming an oblate spheroid. For the WTTSs we simulate here, $v_{\text {crit }} \simeq 276 \mathrm{~km} \mathrm{~s}^{-1}$, and the maximum rotational velocity (at the equator of star) is $u_{\varphi, \max }\left(r_{0}\right)=2 \pi r_{0} / P_{0} \simeq 10,34$, and $202 \mathrm{~km} \mathrm{~s}^{-1}$ for $P_{0}=10,3$, and $0.5 \mathrm{~d}$, respectively. For these three periods, the ratios between the azimuthal and critical velocities are $u_{\varphi, \max }\left(r_{0}\right) / v_{\text {crit }} \simeq 0.04,0.1$, and 0.7. Among the cases we simulate, case $\mathrm{G}$, with $P_{0}=0.5 \mathrm{~d}$ and $u_{\varphi, \max }\left(r_{0}\right) / v_{\text {crit }} \simeq 0.7$, is the case where the star deviates most from spherical symmetry due to rotation. For such case, the ratio of the equatorial radius to the polar radius of the star is $\sim 1.1$ (Maeder and Meynet 2000). This effect is not considered in our simulations.

The adopted grid in our simulations is described in $§ 3.2 .1$ with the initial and boundary conditions presented in $\S 3.2 .2$ and $\S 3.2 .3$, respectively. These conditions propagate in the numerical grid until the steady-state condition is found. The results presented next correspond to when steady state has been achieved.

\subsection{The Magnetic and Wind Structures}

In Fig. 6.1 we present meridional plots for cases A, E, and F. The plots show contours of the poloidal velocity, the black lines are streamlines of magnetic fields, and the white lines represent the Alfvén surface (where the poloidal velocity of the wind equals the local Alfvén velocity). What differs between these three cases is the coronal base density and, consequently, the plasma- $\beta$, i.e., the ratio of the thermal to magnetic energy densities. For cases $\mathrm{E}, \mathrm{A}$, and F, $\beta_{0}$ (evaluated at the pole) is respectively $1,1 / 25$, and $1 / 100$. Analyzing Fig. 6.1, the immediate conclusion is that the configuration of the magnetic field lines is very different in each case. Case E (Fig. 6.1a) presents a dipolar-like configuration of the magnetic field lines. In case F (Fig. 6.1c), the open field lines are more collimated in the polar region and, in the equatorial region, we observe the formation of streamers. In the intermediate case A (Fig. 6.1b), we find a larger region of closed field lines, coexisting with an open field line region emanating from high latitudes of the star. 

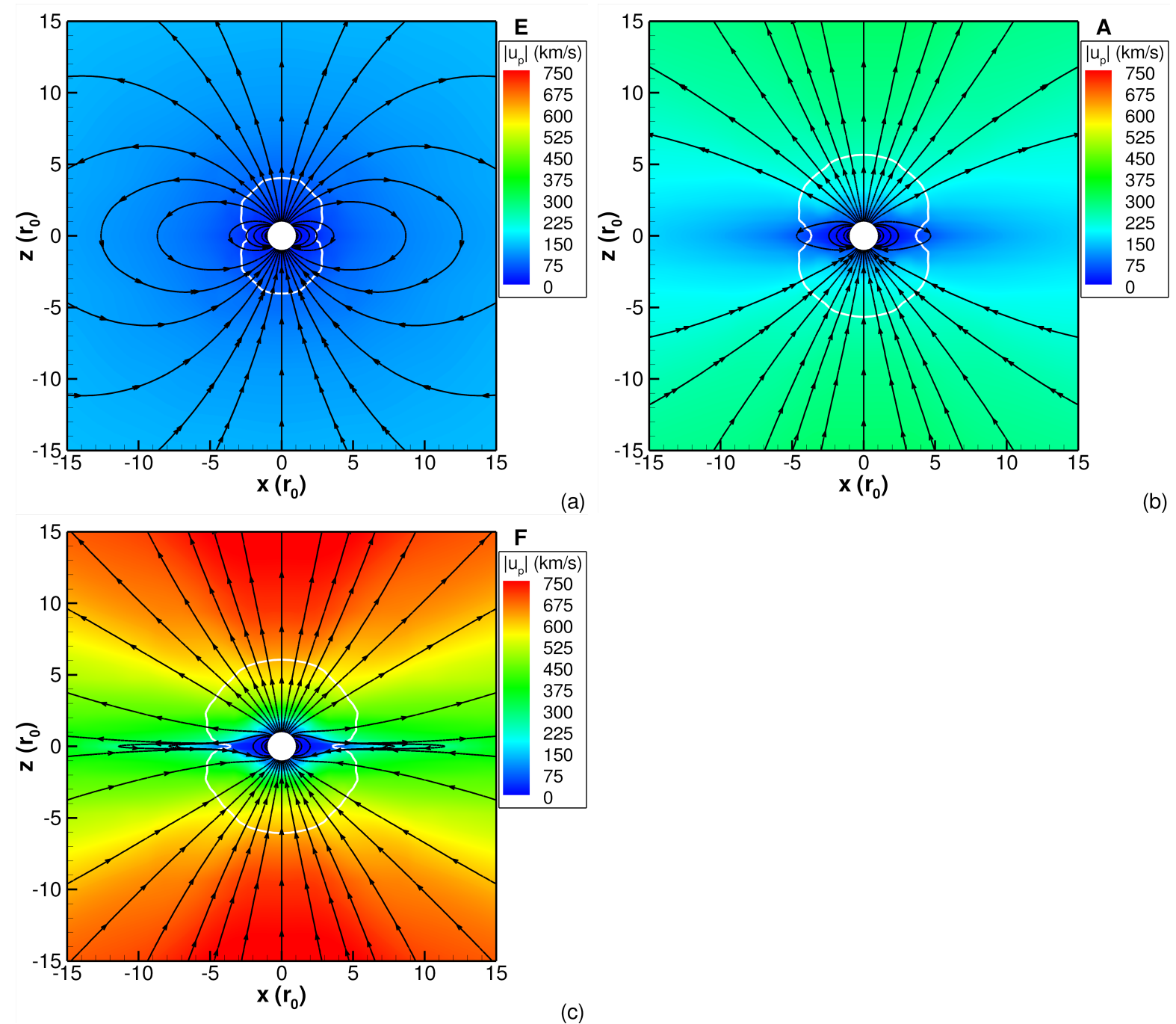

(b)

Figure 6.1: Meridional cuts of poloidal velocity profiles for: (a) case $E\left(\beta_{0}=1\right)$, (b) case $A$ $\left(\beta_{0}=1 / 25\right)$, and $(c)$ case $F\left(\beta_{0}=1 / 100\right)$. The black lines are streamlines of magnetic field and the white lines represent the Alfvén surface.

As discussed in Chap. 4 (Vidotto et al. 2009a) in the context of non-rotating MS solar-like stars, the plasma- $\beta$ is a decisive parameter in the acceleration of the wind. When the magnetic energy density is of the same order as the thermal energy density, as in case E (Fig. 6.1a), the magnetic configuration remains approximately that of a dipole. Otherwise, when the magnetic energy density dominates over the thermal energy density, the wind is accelerated reaching a situation where the ram pressure of the flow is able to distort the initial magnetic configuration, as in case F (Fig. 6.1c).

In addition to the acceleration of the flow, as a consequence of the Lorentz force, the wind velocity becomes more anisotropic with the decrease of $\beta_{0}$. Among the cases we 
analyzed, case E (and also B) presents velocity profiles resembling spherical symmetry and in case $\mathrm{F}$, where $\beta_{0}=1 / 100$ (high magnetic energy density), the wind is noticeably divided in a region of fast speeds at high latitudes and slow speeds at low latitudes.

A comparison between cases A and F, where open field lines are found, shows that (i) case $\mathrm{F}$ shows higher collimation of lines toward the axis of rotation; and (ii) the ratio of open to closed field line is larger in case F. As the magneto-centrifugal forces are responsible for the collimation of lines along the axis of rotation, a wind more magnetically dominated presents higher levels of collimation (considering the same stellar rotational rate). The reason why there are more open magnetic field lines in case $\mathrm{F}$ is mainly due to the flow speed: the high wind velocities stretch and open the previously closed field lines. As the polar wind is faster in case $\mathrm{F}$, it is natural to expect a higher ratio of open to closed field line when compared to case A.

These results also show that longer streamers will be present only if $\beta_{0} \ll 1$. The presence of elongated magnetic features has been recently considered to explain observations of recurrent radio flares in binary systems composed of T Tauri stars (Massi et al. 2008, Skelly et al. 2008). In these systems, the elongated magnetic features belonging to both stars could overlap in certain orbital phases, resulting in a modification of the magnetic configuration (reconnection). This picture could account for giant radio flares, emitted when the binary system is near periastron. Taking for example the system under consideration in Massi et al. (2008, Fig. 1.6), the existence of elongated magnetic features indicates that $\beta_{0} \lesssim 0.01$. Adopting $B_{0} \sim 2600 \mathrm{G}$ and $T_{0} \sim 1 \mathrm{MK}$, it results in $n_{0} T_{0} \lesssim 2 \times 10^{19} \mathrm{~K} \mathrm{~cm}^{-3}$, or $\rho_{0} \lesssim 3.3 \times 10^{-11} \mu \mathrm{g} \mathrm{cm}^{-3}$, where $n_{0}$ is the number density at $r_{0}$ and $\mu$ is the mean molecular weight of the wind particles. Therefore, the observations of radio flares can help us constrain the coronal density in these stars.

PMS stars exhibit X-ray flares occurring in star sized, small structures, often interpreted as scaled-up solar-like coronal activities (Preibisch et al. 2005). In addition, intense X-ray flares are also observed. Favata et al. (2005) analyzed these intense Xray flare decays to model physical parameters of the flaring structure, finding that these large flares take place in very long magnetic structures, extending out to several stellar radii. Getman et al. (2008) also argued that in fast rotating stars these powerful X-ray flares extend beyond the corotation radius, where centrifugal forces dominate over gravity. They conclude that the flares arise in traditional solar-type magnetic loops, where both footpoints are anchored in the stellar surface. Jardine and van Ballegooijen (2005) 
developed a model for magnetic loops in rapidly rotating MS stars that explains the existence of magnetic prominences that can extend out to heights of 2 to $5 r_{0}$, above the corotation radius, in equilibrium with the open field region. In our simulations, we observe an extended, slender closed field region in case F. In this case, the extension of the streamer is assured up to an equatorial radius of $\sim 13 r_{0}$. Above this point, the grid changes resolution, causing a local numerical reconnection (in principle, the loop could extend farther out). At the equatorial plane, the corotation radius is given by

$$
r_{\mathrm{co}}=\left(\frac{G M_{\star} P_{0}}{4 \pi^{2}}\right)^{1 / 3}=4.2 R_{\odot}\left(\frac{P_{0}}{1 \mathrm{~d}}\right)^{2 / 3}\left(\frac{M_{\star}}{M_{\odot}}\right)^{1 / 3},
$$

which for the WTTSs simulated here is $r_{\mathrm{co}} / r_{0}=1.95 P_{d}^{2 / 3}$, where $P_{d}$ is the stellar rotational period measured in days. For $P_{0}=3 \mathrm{~d}, r_{\mathrm{co}} \simeq 4 r_{0}$. This shows that the elongated streamers, as obtained in case $\mathrm{F}$, is stable to at least $\sim 3$ times the corotation radius.

Figure 6.2 shows the steady-state solutions for the remaining of the simulations presented in Table 6.1. We enumerate the following conclusions. (i) We show that only a change in the rotation of the star does not provide a significant change in the poloidal velocity profile of the system (compare cases A and D, F and G). It changes, however, how the magnetic field lines are twisted: the faster is the rotation of the star, the larger is the $\varphi$-component of the magnetic field. ( $i i)$ The heating parameter $\gamma$ influences the thermal acceleration of the wind (compare cases $\mathrm{A}$ and $\mathrm{C}$ ), being more accelerated in the case where the parameterized energy input is higher, i.e., for low $\gamma$. As the wind characteristics are different, the resultant interaction with the magnetic field is different: case A (high $\gamma$ ) presents a larger ratio of open to close lines than case $\mathrm{C}$ (case $\mathrm{C}$ has a larger closed field line region). (iii) Cases B and E present the same characteristics, as they possess the same $\beta_{0}$ (Vidotto et al. 2009a, Chap. 4). Furthermore, because $\beta_{0}=1$, there is an equipartition of thermal and magnetic energy densities at the base of the corona, which results in lower velocities and field lines that are merely modified in relation to the initial configuration, resembling a dipole. (iv) Case $\mathrm{G}$ represents a star with a high rotation rate. Because of this, among the cases we analyzed, it is the case where the open field lines are more collimated toward the axis of rotation. Comparing it to case F, where the rotation rate is six times smaller, it can also be seen that the higher the rotation rate is, the less elongated are the closed field lines.

The Alfvén surface plays an essential role in the determination of the magnetic field configuration. In magneto-centrifugal winds, both $B_{\varphi} / B_{r}$ and $B_{\varphi} / B_{\theta}$ are expected to be 

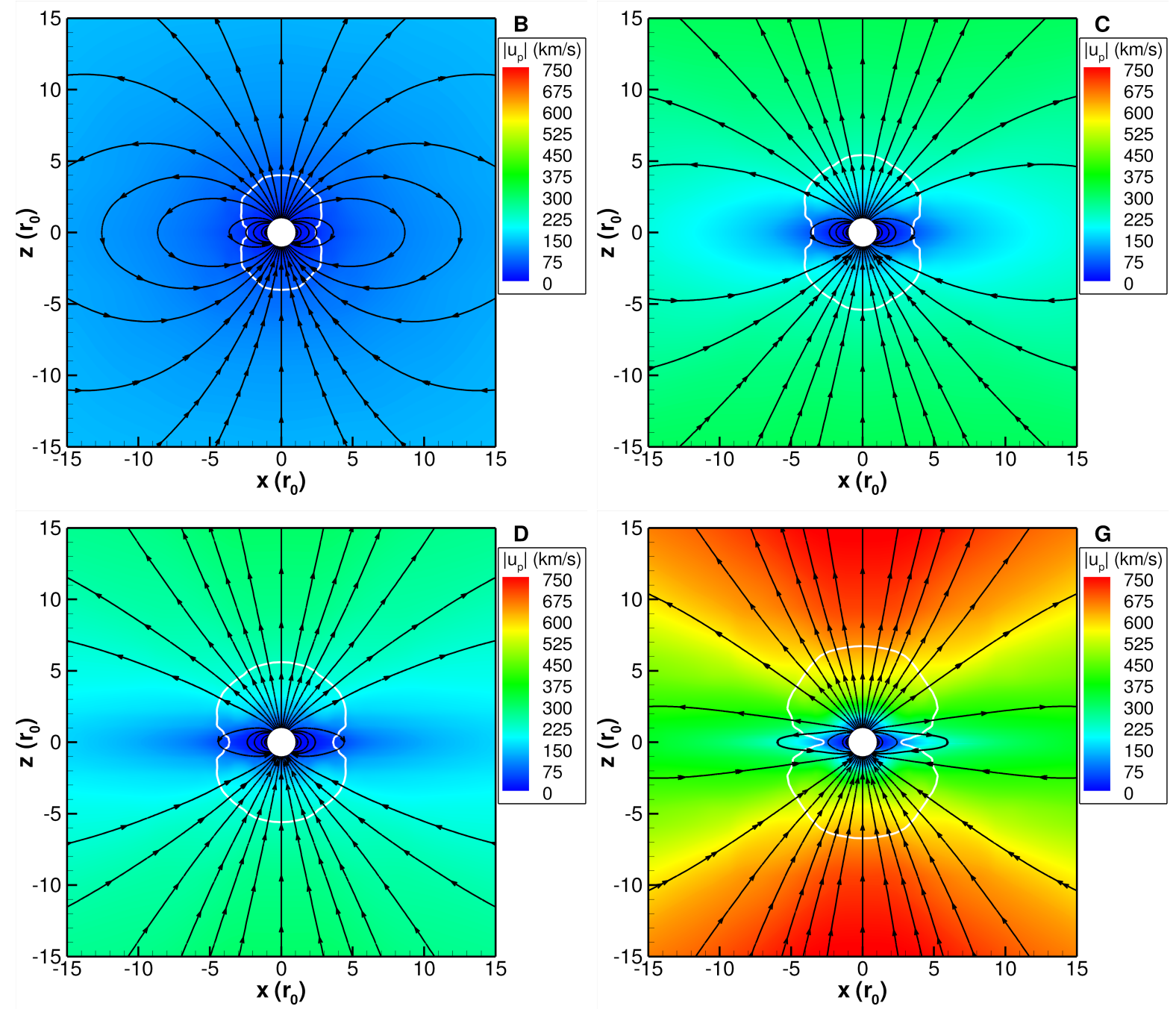

Figure 6.2: Same as Fig. 6.1 for the remaining cases.

small inside the Alfvén surface. Beyond the Alfvén surface, the $r$ and $\theta$ components of the magnetic field decay faster than the $\varphi$ component, causing the field lines to be twisted. Therefore, helmet streamers anchored on rotating stars are not radial. In Fig. 6.3, we plot a $3 \mathrm{D}$ view of the region close to the star. As can be seen, the picture of approximately radial streamers, as observed in the Sun or in slowly rotating stars, is not anymore verified in WTTSs with high rotation rates: both open and closed lines become twisted due to the stellar rotation.

Andre et al. (1988) presented an analytical expression for the calculation of the Alfvén surface, considering that (i) the wind should have spherical mass loss; (ii) by the time the wind is crossing the Alfvén surface, it has already achieved the terminal velocity; and 


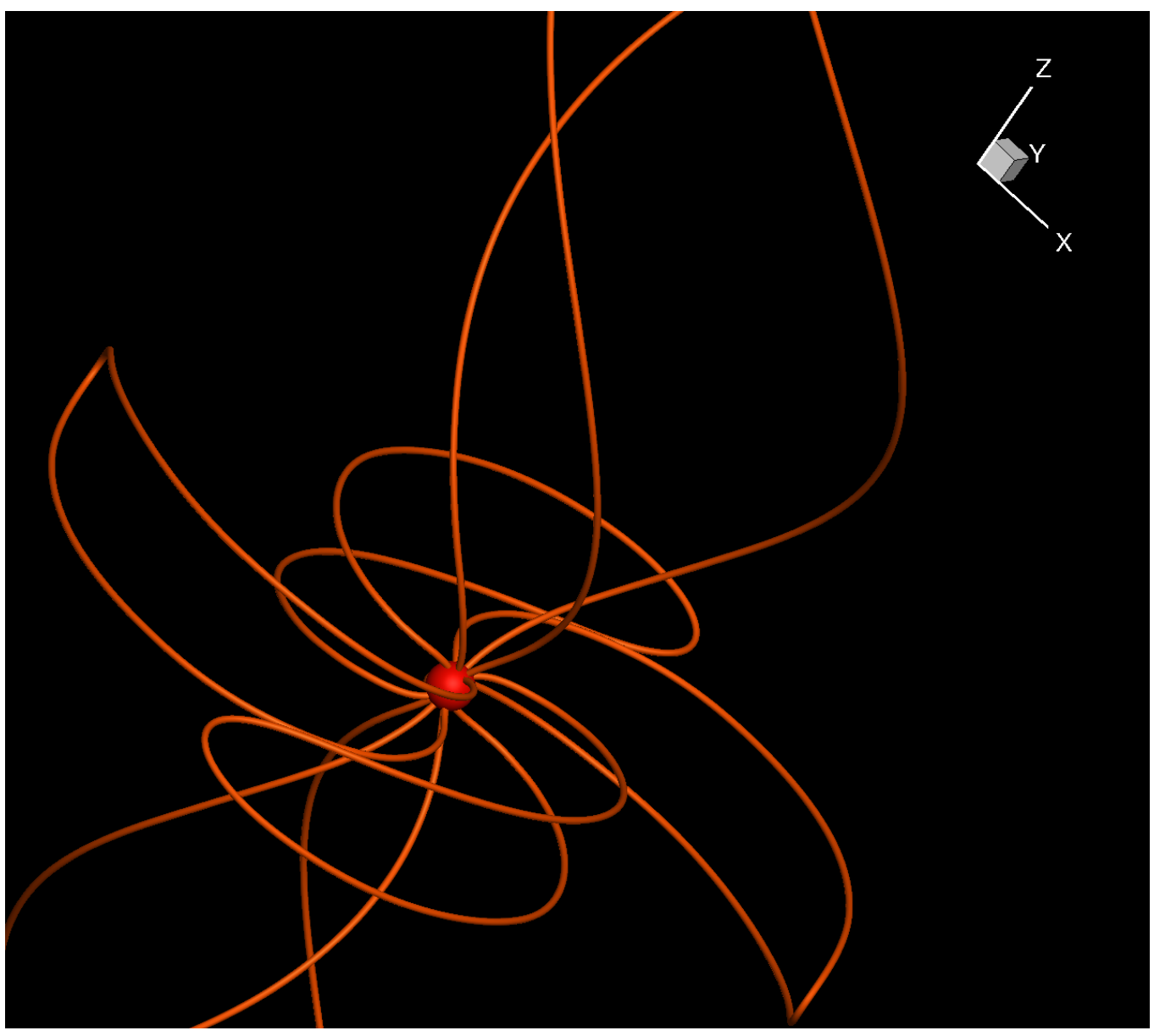

Figure 6.3: Three-dimensional view of the inner most region of case G. The lines plotted were selected as to outline the twisting of both closed and open magnetic lines anchored on the surface of the star.

(iii) the field is dipolar all the way to the Alfvén surface. As can be seen from our results, when the self-consistent interaction of the field lines and the wind is taken into account, these hypotheses may not be verified any longer.

Massi et al. (2008) computed the Alfvén radius of the wind of each of the stars in the binary system V 773 Tau A, where they observed helmet streamers, using Andre et al. (1988)'s analytical expression. They ruled out the twisting of magnetic lines of the helmet streamer, because in their estimate they found a large Alfvén surface with radius of $\sim 60 r_{0}$. According to our model, the Alfvén surface of each individual stellar wind is located much closer to the star and is probably even smaller than the minimum separation of the stars in the binary system V 773 Tau A $\left(\sim 30 r_{0}\right)$, suggesting that the interacting streamers are probably twisted, as illustrated in Fig. 6.3. 


\subsection{Stellar Wind Effects on Planet Migration}

In the previous section, we investigated the magnetic configuration of the wind of WTTSs. This configuration arises naturally from the interplay between the outflow and the field, i.e., the magnetic field is not restricted in our simulations. Several models assume a fixed magnetic field topology, as is the case of the Weber and Davis (WD) model (§1.3.2). In this section, we investigate the effects the wind computed by our more realistic wind model will have on the timescale for migration of a giant planet.

According to the current favored planetary formation theory, the core of a giant planet forms inside the accretion disk, far away from the central star, beyond the snow line ( $\gtrsim 5$ AU, where the disk temperature falls below the condensation temperature of water) by coalescence of planetesimals (Pollack et al. 1996). When the solid core is sufficiently massive, it captures the surrounding gas of the accretion disk to form the atmosphere of a giant planet (Papaloizou and Nelson 2005). However, observations show the existence of planets orbiting solar-like stars at very close distances $(\lesssim 0.1 \mathrm{AU})$. For this reason, these giant planets are often referred as hot-Jupiters. A possible explanation for this inconsistence is that the giant planet may have formed several AU from the central star, and later migrated inward. The interaction of the protoplanet with the disk wherein it was formed is a probable process that may drive this migration (Lin et al. 1996).

The inward migration is expected to cease when the planet is in the inner magnetospheric cavity, a cleared region between the stellar surface and the inner region of the accretion disk (Lin et al. 1996, Trilling et al. 1998, Papaloizou 2007). In recent numerical simulations, Romanova and Lovelace (2006) found that for a stellar dipolar field that is not highly misaligned with the spin axis of the star and neither almost aligned so that 3D instabilities cannot fill the magnetospheric cavity with matter, the formation of the cleared region is assured. As in the cavity there is insufficient material to remove the planet's angular momentum, the giant planet radial motions halt. Also, due to the lack of external material, the planet cannot grow further. In addition to this mechanism, others have been proposed to explain why the planet migration is inhibited all the way to the star (Lin et al. 1996, Fleck 2008).

In this section, in particular, we investigate the action of magnetic torques from the stellar wind acting on the planet.

Considering that a $\mathrm{T}$ Tauri star has magnetic fields and rotation rates that are several 
times larger than in the Sun, Lovelace et al. (2008, LRB08 from now on) studied the torque that a magneto-centrifugally driven wind would cause on a close-in giant planet. They adopted the model of WD for the stellar wind and evaluated the change of the planet's angular momentum due to an azimuthal drag force exerted by the wind on the planet. Considering $M_{\star}=1 \mathrm{M}_{\odot}, r_{0}=2 R_{\odot}, P_{0} \sim 3$ to $5 \mathrm{~d}$, and $B_{0} \sim 1$ to $3 \mathrm{kG}$, they found a timescale of 2 to $20 \mathrm{Myr}$ for a giant planet like Jupiter, orbiting the equatorial plane of the star, to have considerable radial motions.

We estimate this timescale by adopting a more realistic stellar wind, which is obtained from our simulations. Following LRB08, we consider a planet of mass $M_{p}$, orbiting very close to the star at a distance $r_{p}$. The angular momentum of the planet is then $L_{p}=M_{p} v_{K} r_{p}$, where $v_{K}=\left(G M_{\star} / r_{p}\right)^{1 / 2}$ is the azimuthal velocity of the planet, assumed Keplerian. Therefore, a change in the planet's angular momentum leads to

$$
\left|\frac{\mathrm{d} L_{p}}{\mathrm{~d} t}\right| \simeq \frac{1}{2} M_{p} v_{K} \frac{d r_{p}}{d t} \simeq \frac{1}{2} M_{p} v_{K} \frac{r_{p}}{\tau_{w}}
$$

where $\tau_{w}$ is the timescale for the stellar wind drag that significantly changes the planet's orbit (Lin et al. 1996). Another way to express the torque [Eq. (6.2)] is to multiply the force the stellar wind will exert on the planet by the distance $r_{p}$ (LRB08)

$$
\left|\frac{\mathrm{d} L_{p}}{\mathrm{~d} t}\right| \simeq\left(P_{\mathrm{ram}} A_{\mathrm{eff}}\right) r_{p},
$$

where $P_{\text {ram }}$ is the ram pressure the wind exerts on the planet

$$
P_{\text {ram }}=\frac{1}{2} \rho\left(u_{\varphi}-v_{K}\right)^{2}+\frac{1}{4 \pi} B^{2},
$$

and $A_{\text {eff }}$ is the cross section of the planet that intercepts the stellar wind. In order to take into account the effects that the magnetic field of the planet could have in the drag force of the stellar wind, LRB08 defined an effective cross section of the planet as

$$
A_{\mathrm{eff}}=\max \left[\pi R_{p}^{2}\left(\frac{B_{p}^{2} / 4 \pi}{P_{\mathrm{ram}}}\right)^{1 / 3}, \pi R_{p}^{2}\right],
$$

where $B_{p}$ is the surface magnetic field intensity at the pole of the planet and $R_{p}$ is its radius. The planet is assumed to have a dipolar-field configuration, and planetary mass loss is not considered. From Eqs. (6.2) and (6.3), we find

$$
\tau_{w} \simeq \frac{1}{2} \frac{M_{p} v_{K}}{P_{\mathrm{ram}} A_{\mathrm{eff}}} .
$$


A large $A_{\text {eff }}$ means that the cross section of the planet intercepting the wind is large, and thus, a more efficient drag mechanism (i.e., low $\tau_{w}$ ) is expected. If the wind ram pressure is large, the dragging mechanism is again expected to be important. However, a high $P_{\text {ram }}$ also implies in a small $A_{\text {eff }}$ [see Eq. (6.5)].

The planet is supposed to be in the corotation radius if the system is in a tidal equilibrium state (i.e., synchronized with the stellar rotation). However, Levrard et al. (2009) have shown that, in the majority of the observed transiting extra-solar planets, the star's rotation is not synchronous. Due to this reason, we compute $\tau_{w}$ for a range of equatorial radial distances.

Considering the stellar winds from the simulations A, E, and F, we calculated $\tau_{w}$ at the equatorial plane by assuming that the giant planet has the same mass and radius as Jupiter, and that the planet's magnetic field is $B_{p}=100 \mathrm{G}$ (same parameters adopted by LRB08). In order to perform the comparison of our results with the WD model, we evaluate Eq. (6.6) for both models. We thus compute the velocity and magnetic field profiles of the stellar wind using the WD model. We assume the same parameters at the base of the wind as used in our simulations $\mathrm{A}, \mathrm{E}$, and $\mathrm{F}$, with the difference that $B_{0}$ is the magnetic field intensity at the pole in our model, and in the WD model it is the radial field calculated at the surface. We do the same procedure for the more realistic winds we simulate, i.e., we use the results of the simulations for $\mathbf{u}, \mathbf{B}$, and $\rho$ and evaluate $\tau_{w}$ using Eqs. (6.4) to (6.6). These results are shown in Fig. 6.4, where the solid lines represent the results obtained using our model and the dashed lines for the WD model.

Figures 6.4a and 6.4b show, respectively, the effective cross section of the planet and the ram pressure exerted on the planet for the region inside $\sim 0.1 \mathrm{AU}$ (i.e., $\lesssim 10 r_{0}$ ). It can be seen that when $P_{\text {ram }}$ is large, $A_{\text {eff }}$ is small, and vice versa. In addition, $A_{\text {eff }}\left(P_{\text {ram }}\right)$ calculated from our stellar wind model is larger (smaller) than if it were calculated using the WD model. This is because, for the WD model, $P_{\text {ram }}$ is verified to be essentially magnetic, i.e., the first term of the RHS of Eq. (6.4) is negligible compared to the second one, while both terms are significant in our stellar wind model. In addition, for both models, the first term of the RHS of Eq. (6.4) are of the same order. The difference, thus, comes from the magnetic term of Eq. (6.4), which is larger in the WD model than in our model. This behavior is illustrated in Fig. 6.5, for case F.

This results in larger timescales as compared to the WD model (Fig. 6.4c), which implies that the more realistic winds simulated here are not expected to have significant 

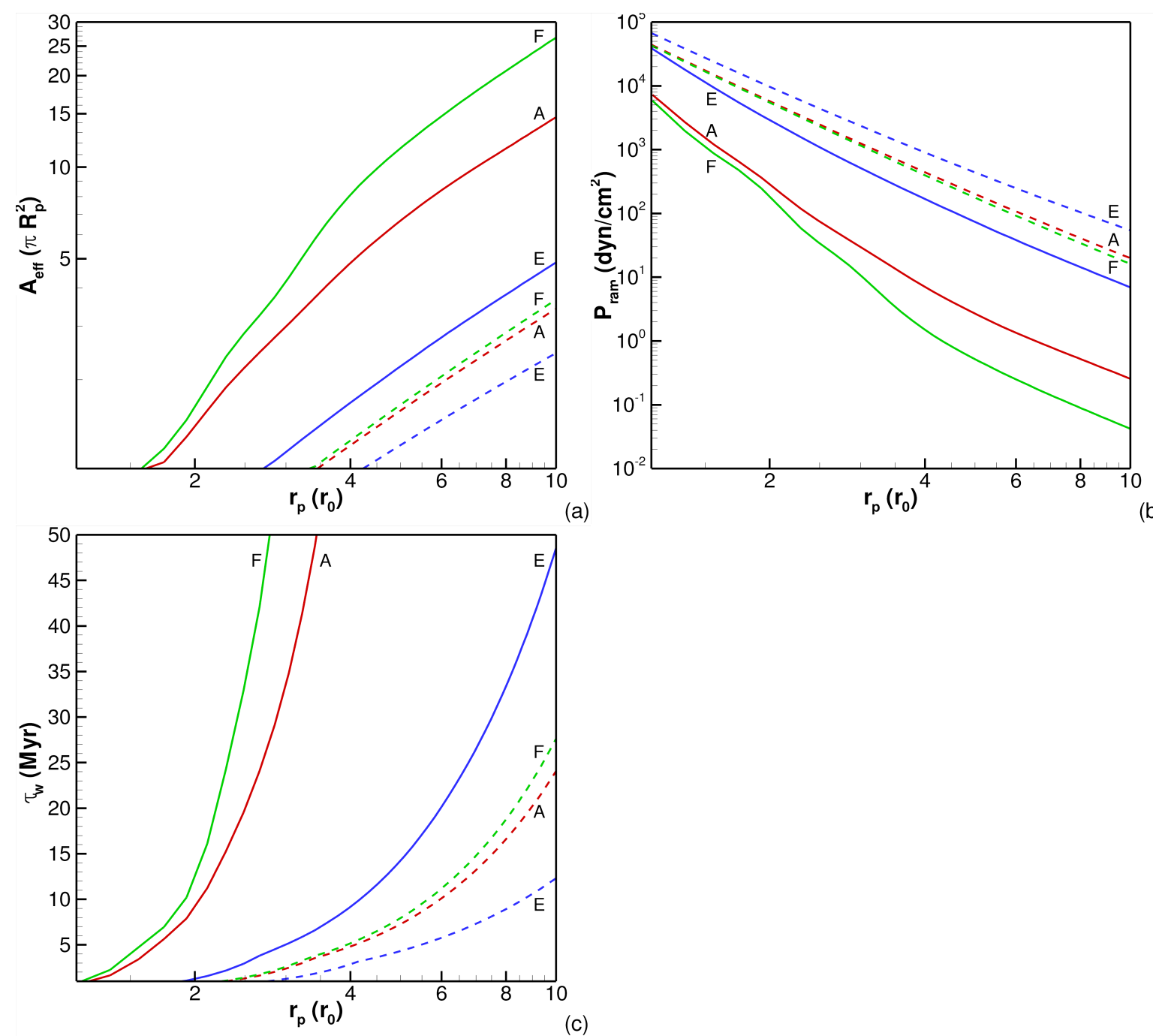

(b)

(c)

Figure 6.4: Comparison of: (a) the effective cross section of the planet that intercepts the stellar wind; (b) the wind ram pressure; and (c) the timescale for the stellar wind drag that significantly changes the planet's orbit. We use the same parameters as in cases $A$ (red lines), E (blue lines), and F (green lines): solid lines are the results for our stellar wind model, and dashed lines for the WD model.

influence on hot-Jupiter migrations. The timescales we estimate (e.g., $\gtrsim 50$ Myr for cases $\mathrm{A}$ and $\mathrm{F}$, for $\left.r_{p} \gtrsim 3 r_{0}\right)$ are much larger than the ones estimated by LRB08 $(\sim 2$ to $20 \mathrm{Myr}$ ). Other effects, such as an increase in the base density or magnetic field intensity, can reduce $\tau_{w}$. Case $\mathrm{E}$ presents the highest base density, while case $\mathrm{F}$ presents the lowest one among our simulations. From Fig. 6.4c, we note that an increase in $\rho_{0}$ reduces $\tau_{w}$. We expect that for $\rho_{0}>2.4 \times 10^{-10} \mathrm{~g} \mathrm{~cm}^{-3}$ with the remaining parameters as adopted in cases A, E, and F, the effects of the migration will be more significant. A similar trend, 


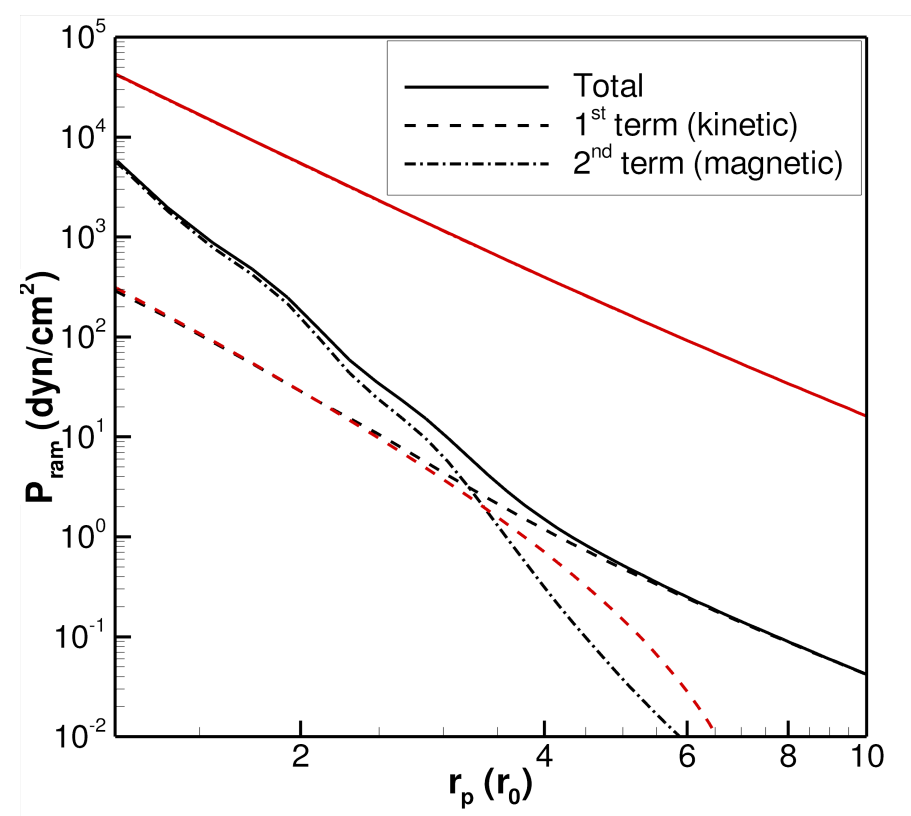

Figure 6.5: Relative importance of the first (dashed lines) and second (dot-dashed lines) terms in the RHS of Eq. (6.4) calculated for case F using the results of our simulations (black) and using the WD model (red). Solid lines are the total ram pressures, i.e., the sum of first and second terms.

i.e., a decrease in $\tau_{w}$, is also expected if one increases $B_{0}$. For example, cases $\mathrm{A}, \mathrm{E}$, and $\mathrm{F}$ assume $B_{0}=1 \mathrm{kG}$. If $B_{0}$ were increased, it would result in a larger ram pressure exerted by the wind on the planet. This would cause a more efficient drag and, ultimately, it would lower $\tau_{w}$ and, perhaps, $\tau_{w}$ would even become smaller than $20 \mathrm{Myr}$ in the region where $r_{p} \lesssim 10 r_{0}$. In the case of higher $B_{0}$, the winds simulated by our model could have a more significant influence on the giant planet migration, although the resultant $\tau_{w}$ would still be much larger than the one computed with the WD model.

According to LRB08, the magnetic winds are expected to be important for planet migration in the case of a multi-polar magnetic field rather than a dipolar field. Here, we extend this statement to the case of the multi-field component coronae, as the ones analyzed previously, where open and closed field lines coexist. Our model suggests that the stellar wind of these modified dipole coronae are not expected to have significant influence on hot-Jupiters migration.

The variation of the angular momentum of planet $\mathrm{d} L_{p} / \mathrm{d} t \propto \operatorname{sign}\left(u_{\varphi}-v_{K}\right)$, which means that the stellar wind causes outward migration of the planet when $u_{\varphi}>v_{K}$, and inward migration when $u_{\varphi}<v_{K}$. In all the simulations we presented, $u_{\varphi}>v_{K}$ only for 
case $\mathrm{G}$, for $r_{p} \gtrsim 1.26 r_{0}$. For all the other cases $u_{\varphi}<v_{K}$, and the wind would cause the planet to move inward, and the closest the planet is from the star, the stronger/faster would be this dragging.

An aspect that is not investigated in this work is when the surface dipole is tilted with respect to the stellar rotation axis. The wind ram pressure is an interplay between magnetic $\left(\propto B^{2}\right)$ and kinetic terms $\left(\propto \rho\left[u_{\varphi}-v_{K}\right]^{2}\right)$. In the tilted case, a planet orbiting the rotational equator would be subjected to high magnetic field intensities (since $|\mathbf{B}|$ is maximum at the magnetic pole and minimum at the magnetic equator). However, it is unclear the latitudinal dependence of $u_{\varphi}$ : in the aligned case, $u_{\varphi}$ is maximum at the equator; in the misaligned case and in a highly magnetized ambient, the stellar magnetic field is expected to channel the rotating outflow. Hence, by a superposition of the magnetic and kinematic effects, it could be possible that $\tau_{w}$ becomes smaller in a tilted configuration. Future works will explore this scenario.

Another interesting configuration is when the orbital axis of the planet is inclined with respect to the spin axis of the star, as it is believed to occur, e.g., in the extra-solar planets HD80606b (Moutou et al. 2009) and XO-3b (Hébrard et al. 2008, Winn et al. 2009). In this configuration, the planet would interact with a stellar field strength that is larger at the position of the planet than at the magnetic equator, which could have an effect on $\tau_{w}$.

\subsection{Discussion and Conclusion}

Recent works indicate the presence of complex magnetic structure, as the one observed in the Sun, in T Tauri stars. This structure certainly influences the stellar winds. WTTS offers a tool for studying the magnetic behavior of low-mass PMS stars with the benefit that it is no longer strongly influenced by the presence of an accretion disk. As a consequence, the study of WTTS may eventually have applications on theories of CTTSs and also clues on the physical conditions for planetary formation and/or migration.

In the present work, we showed 3D MHD numerical simulations of magnetized stellar winds in rotating $\mathrm{T}$ Tauri stars. With the parameters we selected, we scan the possible values of rotational period ( 0.5 to $10 \mathrm{~d}$ ), magnetic field intensities (200 to $1 \mathrm{kG}$ ), and surface density $\left(2.4 \times 10^{-12}\right.$ to $\left.2.4 \times 10^{-10} \mathrm{~g} \mathrm{~cm}^{-3}\right)$. We show that the plasma- $\beta$ parameter 
is a crucial parameter in the acceleration of the wind and in shaping the magnetic field lines. If the surface magnetic and thermal energy densities are equal at the pole, i.e., $\beta_{0}=1$, we showed that (i) the wind presents approximately spherical symmetry; (ii) the magnetic field lines are not significantly distorted from the dipolar field that we assume as an initial condition of the simulation; and (iii) the wind velocity does not differ considerably from the hydrodynamic case. On the other hand, if the magnetic energy density is much greater than the thermal one, i.e., $\beta_{0} \ll 1$, we observed departure from spherical symmetry. The wind shows two different components of the velocity, being faster at high latitudes and slower at low latitudes. In addition, the field lines are not anymore dipolar: the high velocity of the flow is able to stretch and open the field lines at high latitudes, while in low latitudes we observe elongated magnetic features. This indicates that streamer structures should be present in WTTSs with $\beta_{0} \ll 1$.

In a different context, ud-Doula and Owocki (2002) defined a similar parameter that is used as an indicator of the effectiveness of magnetic fields in channeling outflow from hot supergiant stars. The "magnetic confinement parameter", defined by them as the ratio between the magnetic and kinetic wind energy densities, operates in a similar fashion as $\beta_{0}$ operates in our work: it identifies whether the magnetic field will play a significant role in the acceleration of the wind. However, both in the present work and in Chap. 4 (Vidotto et al. 2009a), the thermal energy of the (coronal) wind is more significant then the kinetic energy, while in the line-driven winds studied by ud-Doula and Owocki (2002), the kinetic energy is more important than the thermal energy of the wind.

We also show that when the self-consistent interaction of the field lines and the wind is taken into account, the picture of approximately radial streamers (near the star), as observed in the Sun, is not anymore verified for the WTTSs with rotational periods up to $10 \mathrm{~d}$ : both open and closed lines are twisted.

With typical parameters of WTTSs, we estimate the timescale for planet migration due to the torque exerted by the azimuthal ram pressure of the stellar winds, and we showed that for the multicomponent corona of a WTTS simulated here, this migration mechanism is not important, in contrast to the results from LRB08, who argued that magnetic winds computed using the WD model are expected to be important for planet migration. Further simulations with a greater parameter range (higher magnetic field intensity or higher density at the coronal base) or different system configurations (a tilted stellar magnetosphere or a tilted planetary orbit with respect to the stellar spin 
axis) may reduce the timescale to significantly affect the orbital radius of the planet. For instance, if we consider a magnetic field intensity of 2 to $3 \mathrm{kG}$, still consistent with values derived from Zeeman-broadening measurements (Johns-Krull 2007), a reduction in $\tau_{w}$ is expected, as the ram pressure of the wind increases. 


\section{Chapter 7}

\section{D Simulations of Misaligned Magnetospheres of WTTSs}

his chapter is motivated by recent surface magnetic field maps of T Tauri stars, where it was found that the orientation of the stellar magnetic moment is often misaligned with the axis of rotation of the star (Donati et al. 2007; 2008a, Hussain et al. 2009). In such configuration, the system loses the axisymmetry presented in the aligned case (Chaps. 4 to 6), requiring a fully 3D approach. In this chapter, we present results from simulations where there is a misalignment between the magnetic moment and the rotation vectors and study how such misalignment influences the stellar wind. The model is once more applied to a T Tauri star, neglecting the action of the disk in the coronal structure of the star. This work extends the study of Chap. 6 (Vidotto et al. 2009b), where the rotation and magnetic moment vectors were assumed to be parallel, along the $z$-axis. The results presented here are preliminary and will be explored in a forthcoming publication.

\subsection{The System Configuration}

The star is considered to be rotating as a solid body with a period of rotation $P_{0}=2 \pi / \Omega$, where $\Omega$ is the angular velocity of the star. Its axis of rotation is along the $z$-direction

$$
\Omega=\Omega \hat{\mathbf{z}}
$$


The stellar magnetic moment vector $\mathbf{m}$ is tilted with respect to $\Omega$ at an angle $\theta_{t}$

$$
|\mathbf{m} \cdot \Omega|=m \Omega \cos \theta_{t}
$$

As the magnetic field is anchored on the star, in one stellar rotational period, the magnetic moment vector $\mathbf{m}$ will draw an imaginary cone, whose central axis is the $z$-axis (Fig. 7.1a).

The simulations are initialized with a dipolar magnetic field described in spherical coordinates $\{r, \theta, \varphi\}$ by

$$
\begin{aligned}
& B_{r}\left(t_{0}\right)=\frac{B_{0} r_{0}^{3}}{r^{3}}\left(\cos \theta \cos \theta_{t}+\sin \theta \cos \varphi \sin \theta_{t}\right), \\
& B_{\theta}\left(t_{0}\right)=\frac{B_{0} r_{0}^{3}}{r^{3}}\left(\frac{1}{2} \sin \theta \cos \theta_{t}-\frac{1}{2} \cos \theta \cos \varphi \sin \theta_{t}\right), \\
& B_{\varphi}\left(t_{0}\right)=\frac{B_{0} r_{0}^{3}}{r^{3}} \frac{1}{2} \sin \varphi \sin \theta_{t},
\end{aligned}
$$

where $B_{0}$ is the magnetic field intensity at the magnetic poles (where $\theta=\theta_{t}$ and $r=r_{0}$ ). At the initial instant $t_{0}$, the vector $\mathbf{m}$ is in the $y=0$ plane, tilted by an angle $\theta_{t}$ in the clockwise direction around the $y$-axis (Fig. 7.1b). The MHD solution is evolved in time from the initial dipolar configuration for the magnetic field to a fully self-consistent non-dipolar solution. The wind interacts with the magnetic field lines and deforms the initial dipolar configuration of the field. The stellar wind is also modified by the magnetic field, i.e., no fixed topologies for the magnetic field neither for the wind are assumed.

Except for the magnetic field, the remaining boundary and initial conditions are the same as presented in $\S 3.2 .3$ and $§ 3.2 .2$, respectively. As the magnetic moment vector $\mathbf{m}$ varies in time, in the misaligned dipole simulations, the inner boundary conditions are time-dependent. For this reason, the simulations never reach steady state. Rather, they achieve a periodic configuration.

In these preliminary simulations, we are adopting a simpler and coarser grid than the one presented in $§ 3.2 .1$. Figure 7.2 presents the inner portion of the grid, where we note that more resolution is concentrated only around the central star, differently to the grid that has been used in the previous chapters, where we refined also around the equatorial plane $(z=0$, compare Figs. 3.3 with 7.2$)$. 

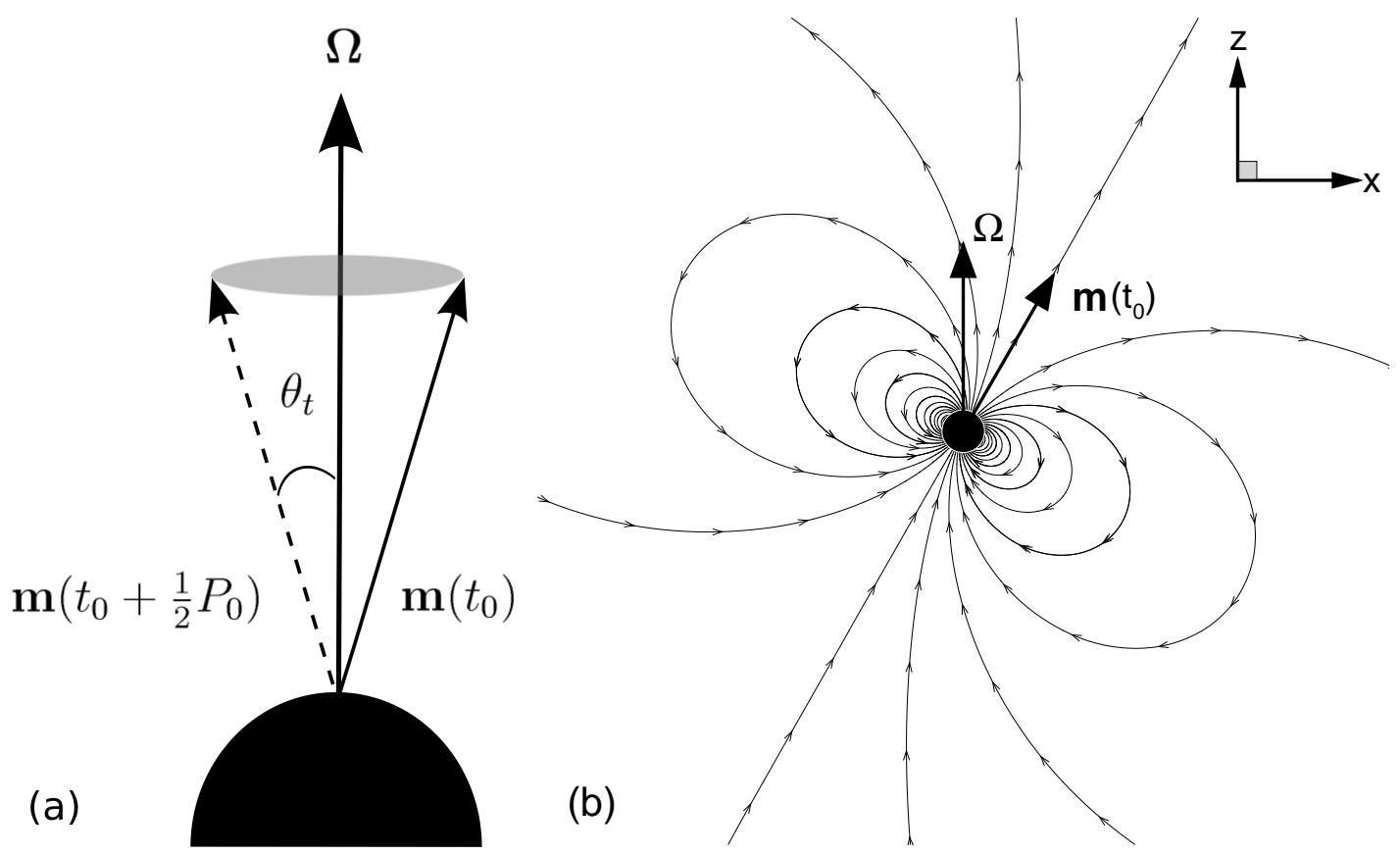

Figure 7.1: The magnetic field configuration in the simulations of misaligned magnetospheres: (a) in one stellar rotational period, the magnetic moment vector $\mathbf{m}$ draws an imaginary cone, whose central axis is the z-axis; (b) orientation of the magnetic field lines in the $y=0$ plane at the instant $t_{0}$.

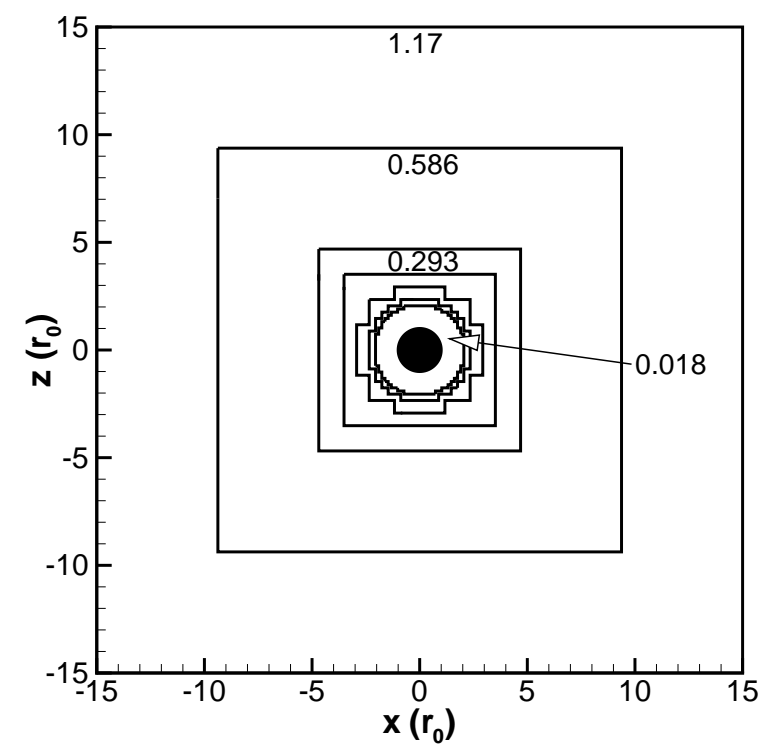

Figure 7.2: Adopted grid in the simulations of misaligned magnetospheres. The numbers represent the maximum size of the cell inside each delimited region (in units of the stellar radius $\left.r_{0}\right)$. 


\subsection{Results}

We present in this chapter, three new simulations, where we have considered the following parameters. The star has $M_{\star}=0.8 \mathrm{M}_{\odot}, r_{0}=2 \mathrm{R}_{\odot}$, and $P_{0}=1 \mathrm{~d}$; for the wind, we adopt $\gamma=1.2$; at the base of the wind, we adopt $T_{0}=10^{6} \mathrm{~K}, \rho_{0}=10^{-11} \mathrm{~g} \mathrm{~cm}^{-3}$, and at the magnetic pole $B_{0}=1000 \mathrm{G}$. These values lead to a plasma- $\beta$ parameter at the magnetic pole $\beta_{0}=1 / 25$. These are essentially the same values as for the case $\mathrm{A}$ presented in Chap. 6, except that here we have a star rotating three times faster. The only parameter that differs between these three new simulations is the misalignment angle $\theta_{t}$, which was chosen to be $\theta_{t}=10^{\circ}, 20^{\circ}$, and $30^{\circ}$.

\subsubsection{Time-Dependent Behavior}

We ran the simulations for $\sim 8$ stellar rotational periods, which is a time interval that is sufficient for the solution at $r_{0}$ to propagate in the grid, and, thus, remove the conditions that were initially set. Steady state is not achieved in the present simulations, rather we find a periodic behavior of the system with the same period of rotation of the star. Figure 7.3 illustrates such time-dependent behavior, where we show meridional cuts of the poloidal velocity of the wind for seven instants during one full period of rotation of the star. The first panel represents a given instant $t=t_{1}$; the subsequent panels represents multiples of $1 / 6 P_{0}=4 \mathrm{~h}$, until the cycle completes at $t=t_{1}+P_{0}$. The magnetic field lines are represented by black lines and the white line represents a contour where the magnetic field changes polarity (i.e., when $B_{r}=0$ ). We note that the instants $t=t_{1}$ and $t=t_{1}+P_{0}$ are identical, which exemplifies the periodic behavior of the simulation. We also note that the magnetic field has zones of open and closed field lines. The zone of closed field lines, for example, oscillates around the equatorial plane $(z=0)$, as is readily seen if one follows the white lines of the plots.

\subsubsection{The Effects of the Misalignment Angle}

Let us now compare the simulations with three misalignment angles $\theta_{t}=10^{\circ}, 20^{\circ}$, and $30^{\circ}$. Figure 7.4 presents poloidal velocities of three snapshots of the simulations after they achieved the periodic behavior described in $\S 7.2 .1$. The three panels shows meridional cuts after 8 stellar rotational periods, and each panel corresponds to a different misalignment 


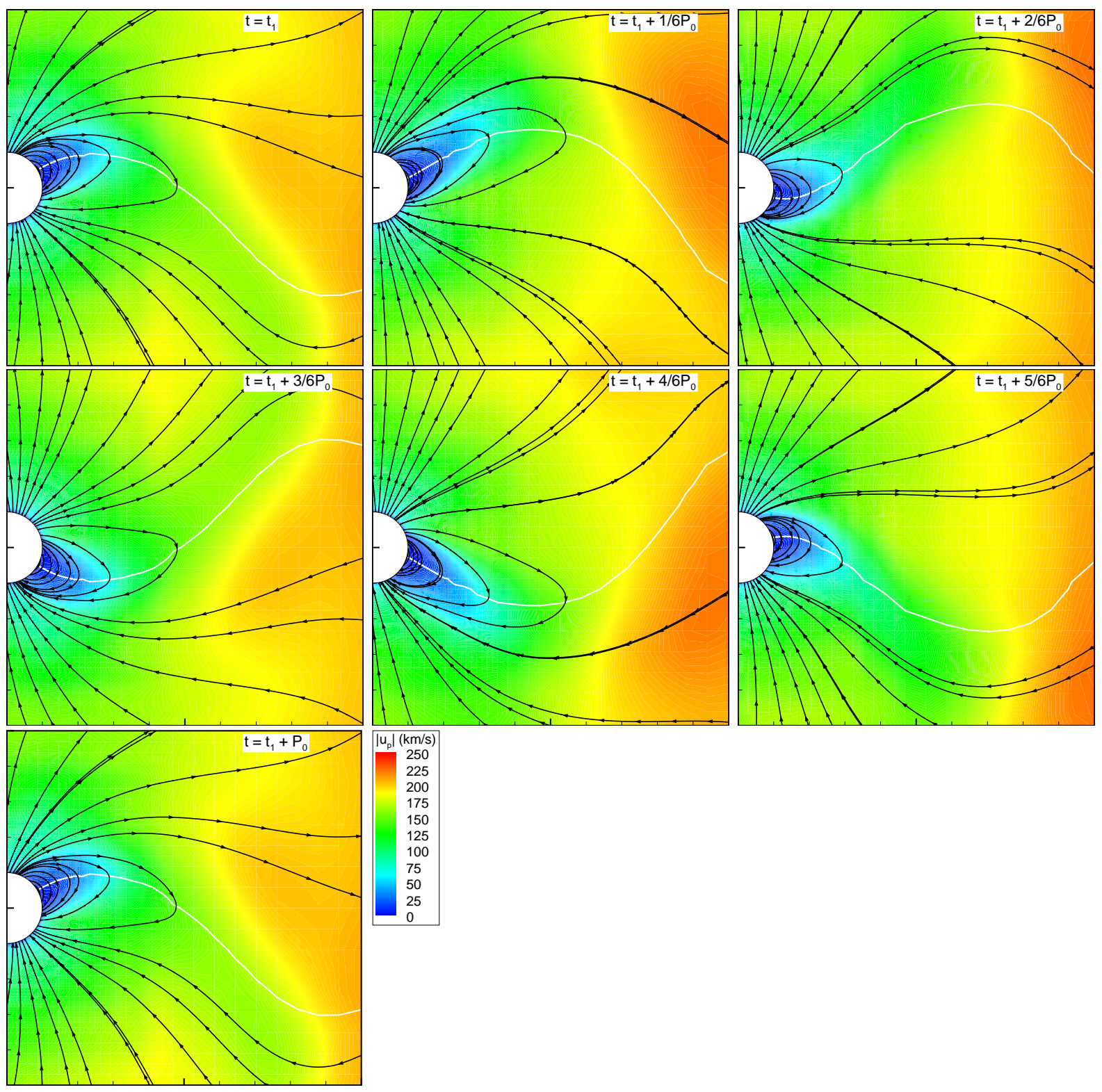

Figure 7.3: Temporal evolution of the simulation with $\theta_{t}=30^{\circ}$ during one full stellar rotational period. The panels show 7 different instants, starting from $t=t_{1}$, and increasing in multiples of $1 / 6 P_{0}=4 h$, until $t=t_{1}+P_{0}$. We plot meridional cuts of the poloidal velocity, the magnetic field lines are represented by black lines, and the white line represents a contour where the magnetic field changes polarity (i.e., when $B_{r}=0$ ). The $x$-axis ranges from 0 to $10 r_{0}$ and the $y$-axis ranges from $-5 r_{0}$ to $5 r_{0}$.

angle analyzed. The magnetic field lines are represented by black lines, and the white line represents a contour where the magnetic field changes polarity (i.e., when $B_{r}=0$ ). 

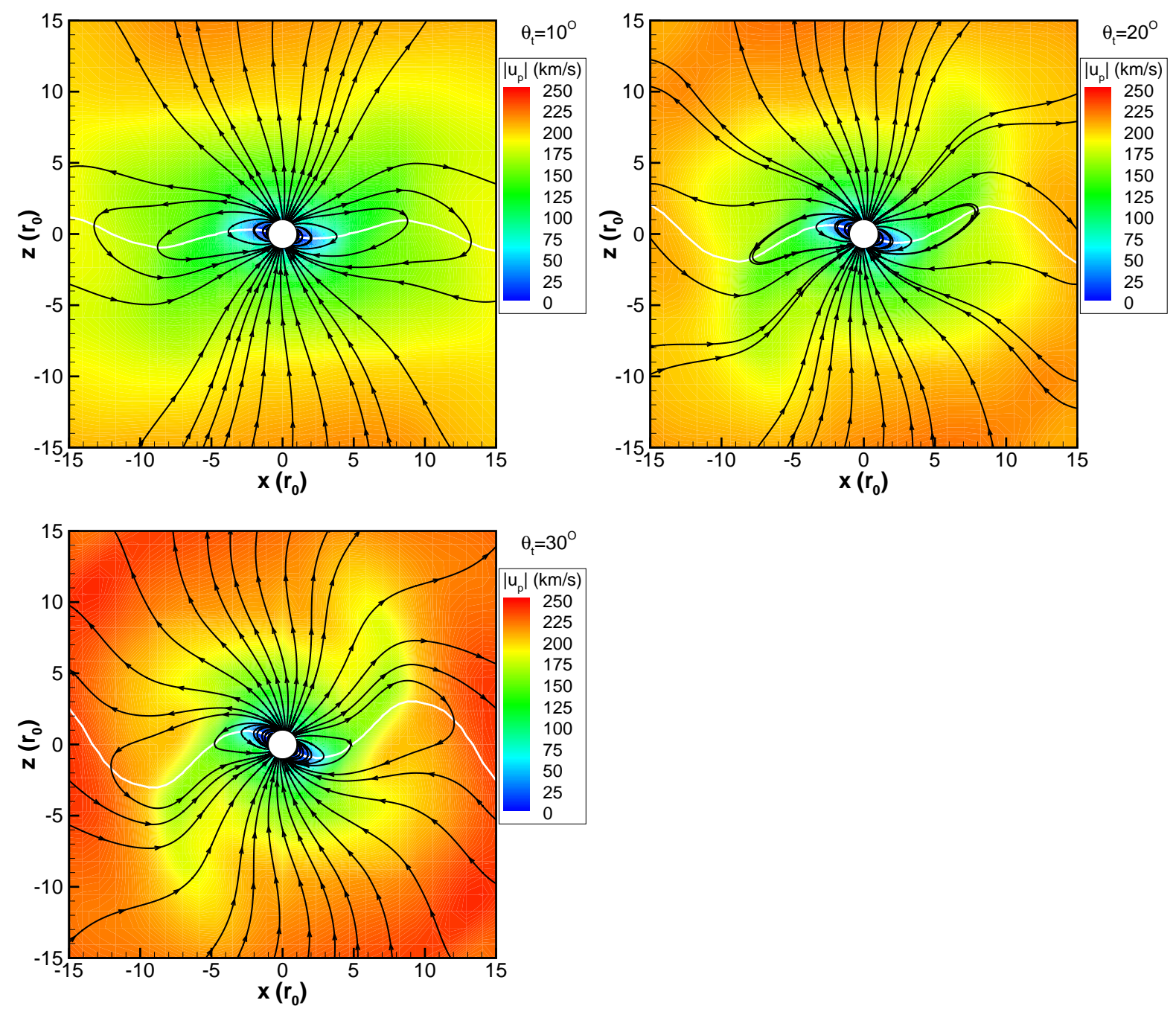

Figure 7.4: Meridional cuts of poloidal velocities for three misalignment angles $\theta_{t}=10^{\circ}, 20^{\circ}$, and $30^{\circ}$. The panels are snapshots of the simulations after 8 stellar periods of rotation. The black lines represent magnetic streamlines and the white lines show contours for $B_{r}=0$ (when the magnetic field changes polarity).

We note that the increase in $\theta_{t}$ leads to faster winds. We will investigate in future works the cause of such behavior. By following the white line, we also note that the magnetic field presents higher amplitude of oscillation for larger $\theta_{t}$ and we note that the white line is concentrated inside a conical aperture that makes an angle $\sim \theta_{t} / 2$ above and below the equatorial plane $(z=0)$.

Figure 7.5 presents zooms of the inner region of the simulations, where panels on the left show azimuthal velocity contours and panels on the right show azimuthal field 

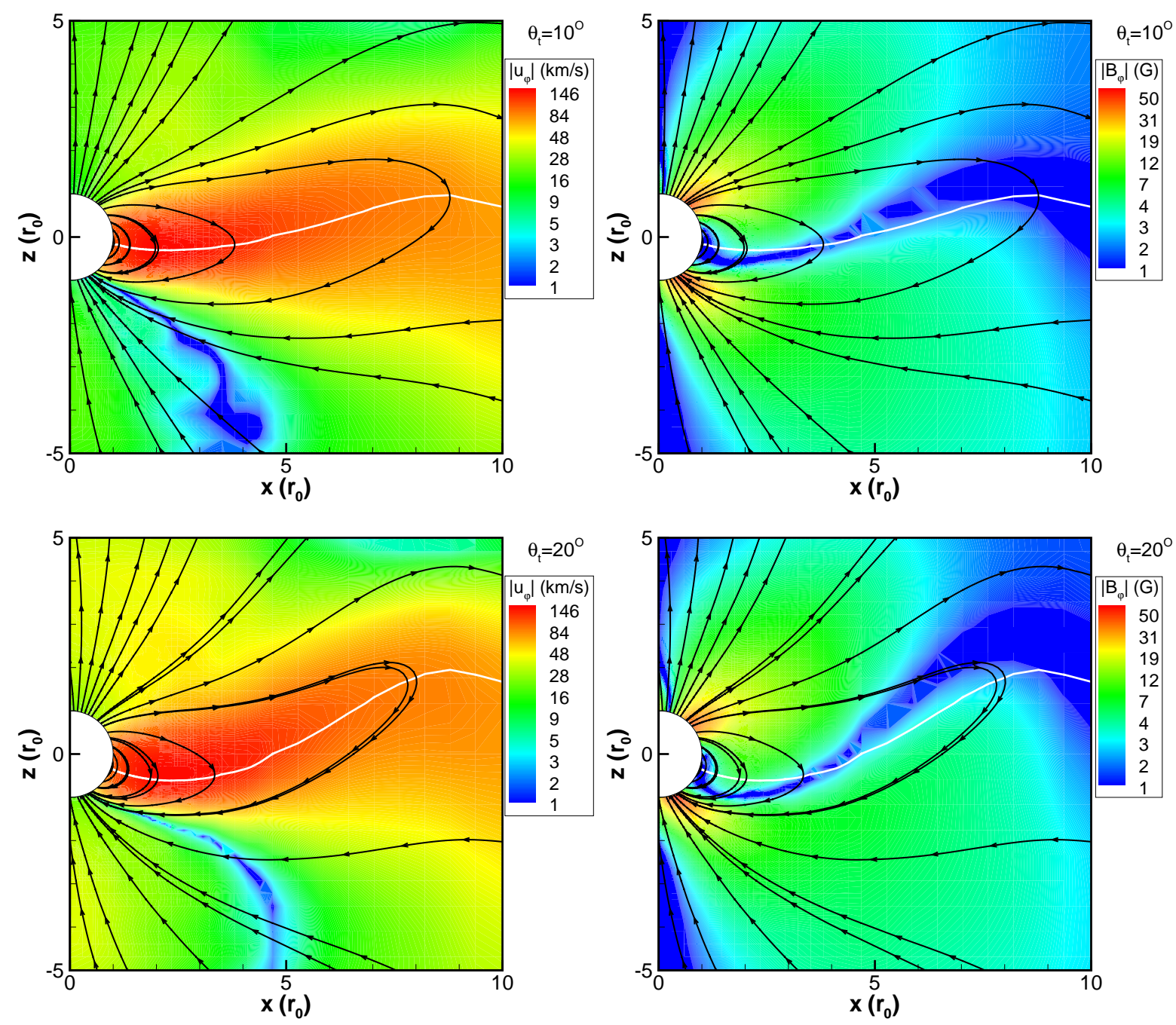

Figure 7.5: Meridional cuts of the azimuthal components of velocity (left) and magnetic field (right) for three misalignment angles $\theta_{t}=10^{\circ}$ (upper row), $20^{\circ}$ (lower row), and $30^{\circ}$ (row in the next page). The instants plotted are the same as presented in Fig. 7.4 and the contours are in logarithmic scale. Continues in the next page.

contours. Each row of panels display a different $\theta_{t}$ analyzed. Black and white lines have the same meaning as before. We note that the azimuthal velocity of the wind is also deflected from the equatorial plane $(z=0)$, which shows that the wind is coupled to the magnetic field lines and tries to follow the oscillatory motion of the stellar magnetosphere. For a rotational period of $P_{0}=1 \mathrm{~d}$, the maximum azimuthal velocity at the surface of the star is $u_{\varphi, \max }\left(r_{0}\right)=2 \pi r_{0} / P_{0} \simeq 100 \mathrm{~km} \mathrm{~s}^{-1}$. However the wind reaches values 

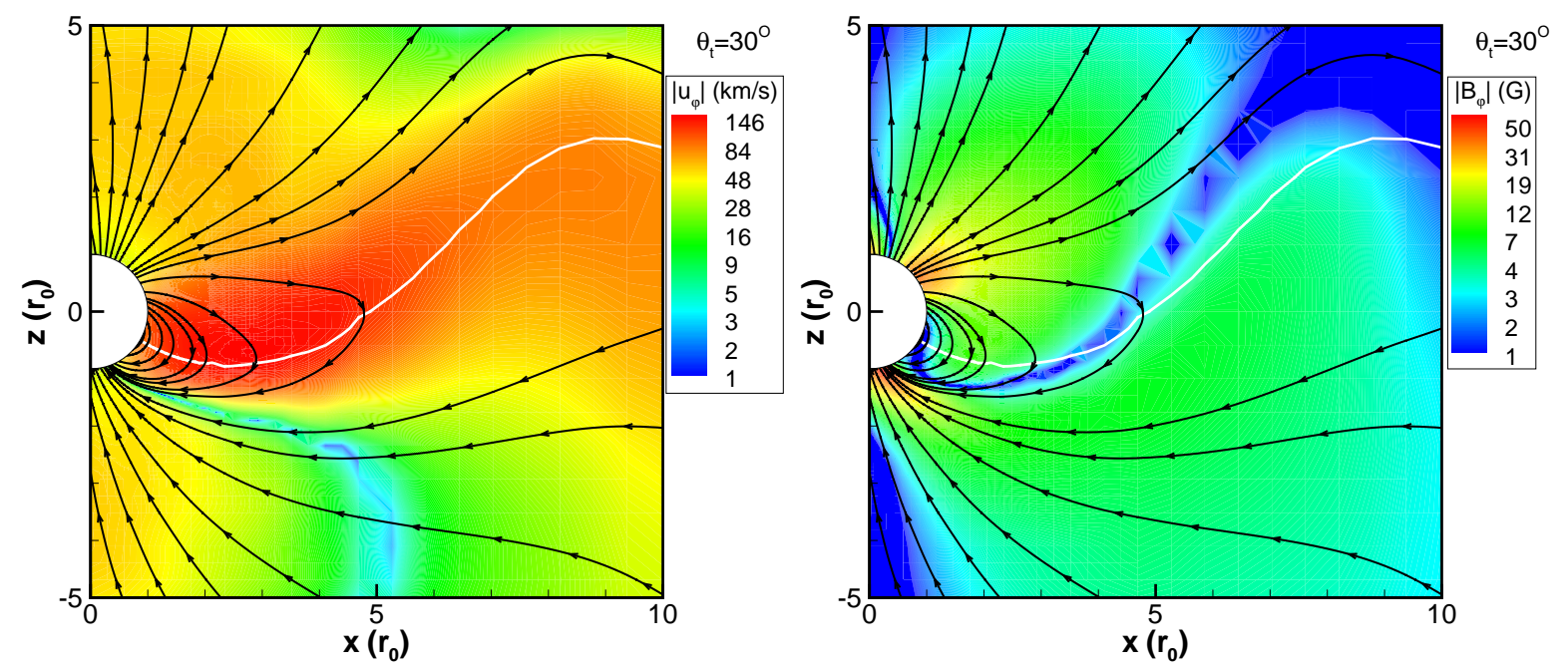

Figure 7.5: Cont.

$\gtrsim 150 \mathrm{~km} \mathrm{~s}^{-1}$, showing that the magnetic field tries to maintain the wind in corotation with the star. Such wind rotation gives rise to an azimuthal component for the magnetic field lines and we see from the panels on the right in Fig. 7.5 that $\left|B_{\varphi}\right|$ is maximum on the interface between closed and open field line regions, near the star.

The periodic movement of the magnetosphere affects the entire wind structure. Figure 7.6 shows, for example, the azimuthal velocity of the wind in the case $\theta_{t}=30^{\circ}$. In

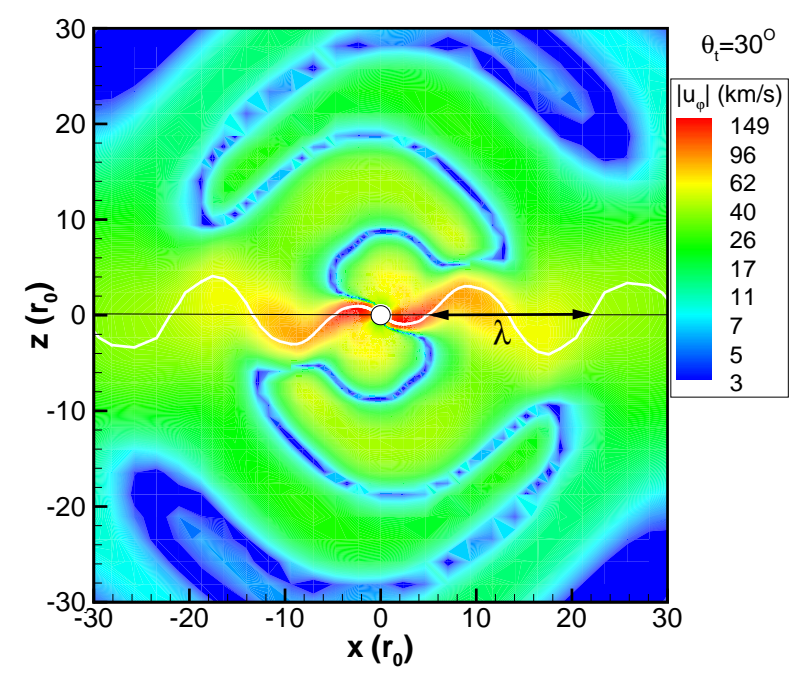

Figure 7.6: Meridional cuts of azimuthal velocity of the wind, as seen from a larger portion of the grid. Contours are in logarithmic scale. 
the case where there is no misalignment between $\Omega$ and $\mathbf{m}, u_{\varphi}=0$ at the polar axes. In the Fig. 7.6, we note that $u_{\varphi}=0$ in a sinusoidal "line". We also can not specify anymore fixed hemispheres with positive/negative polarity. Instead, the white line $B_{r}=0$ oscillates up and down the equatorial plane. The length $\lambda$ (Fig. 7.6) of this oscillation can be estimated as (Lazarian and Opher 2009)

$$
\lambda \simeq u_{\text {char }} P_{0}
$$

where $u_{\text {char }}$ is the characteristic velocity of the wind. As $P_{0}$ is the same for the three simulations performed, it is immediate to conclude that $\lambda$ will be larger for larger $\theta_{t}$, as the velocity of the wind increases with $\theta_{t}$. In fact, our estimates show that $\lambda \sim 13$, 14 , and $16 r_{0}$ for $\theta_{t}=10^{\circ}, 20^{\circ}$, and $30^{\circ}$, respectively, which are consistent to the values measured from the simulations $\lambda \sim 14,16$, and $17 r_{0}$.

\subsection{Final Remarks}

In this chapter, we presented preliminary simulations of magnetized stellar winds where the stellar magnetosphere is tilted with respect to the axis of rotation of the star. This configuration requires a fully 3D approach, as the system does not present axisymmetry. We so far have performed three simulations investigating the effects of the misalignment angle on the wind structure. More precise analyses require a finer grid than the one adopted in these preliminary simulations, although the overall results we presented should not change drastically. With a grid with more resolution, we expect to better define, for instance, the sinusoidal "line" we note in Fig. 7.6, or the blue regions of azimuthal velocity and field in Fig. 7.5. Furthermore, a wider range of parameters should be explored in future works. We would like to investigate, for instance, how the magnetic field intensity can change the wind structure in a highly misaligned magnetosphere. Another interesting topic we could analyze is whether the inclination of the magnetosphere can enhance the angular momentum loss through stellar winds. 


\section{Chapter 8}

\section{Summary of the Thesis and Perspectives}

he magnetic field plays important roles in the acceleration and heating of coronal stellar winds and, consequently, in the determination of the structure of a magnetized wind. For this reason, a realistic determination of the coronal magnetic structure is essential. However, the interplay between the magnetic field and the wind is very complex because, mathematically speaking, the equations of the MHD form a coupled system of first order partial differential equations. In three dimensions, there are eight equations to be solved. Because of that, several approximations have to be adopted, otherwise, numerical methods, which usually require a large computational effort, need to be used.

We have presented in this thesis a stepwise development of a simulational study whose subject was magnetized winds of low-mass stars. Using the numerical code BATS-R-US, we have performed three-dimensional simulations of winds of MS solar-like stars and WTTSs, investigating the coronal magnetic and wind structures.

In the first three chapters, we provided the reader with the necessary base to what was presented in the remainder of the thesis: Chap. 1 presented an overview of coronal winds, including a description of the numerical models developed in the past years. Chapter 2 introduced the equations of magnetohydrodynamics, an approach that treats the plasma as a magnetized fluid. The necessary equations were derived, and their main characteristics were discussed. In Chap. 3 we presented the numerical code BATS-R-US. The code was implemented to study coronal winds of solar-like stars: a grid suitable for 
the problem was developed and described in such chapter; we also presented the physical conditions of the problem; described the boundary conditions used; and presented the criterion to determine the steady-state solution.

In Chap. 4 (Vidotto et al. 2009a) we presented the first numerical simulations of magnetized winds, but we did not take into consideration stellar rotation. A thermally driven coronal wind was set on the grid together with a stellar magnetic dipole. The simulation was turned on and when steady state was achieved, the results were analyzed. We showed that the coronal winds of solar-like stars present a multi-field configuration, with zones of closed and open field lines. In the zone of open field lines, which is located around the polar axes, faster winds were observed. In the equatorial region and closer to the star, we observed a zone of closed field lines and the consequent formation of a helmet streamer, with a cusp on top of it. We simulated 13 cases with different physical conditions (Table 4.1), to explore the effects different plasma- $\beta$ 's have on the wind structure. We also varied the heating parameter $\gamma$ in our simulations. We showed that, for a constant $\gamma$, the structure of the wind (velocity, density, pressure profiles) significantly depends on the ratio of the thermal to magnetic pressures at the base of the wind $\left(\beta_{0}\right)$ : for small $\beta_{0}$ (i.e., magnetic energy densities more important) the wind becomes more accelerated.

In Chap. 5 we presented a way to mimic energy deposition in the wind. We do not know exactly how the different energetic mechanisms act on the wind. However, from observations of the solar wind, there are reasons to believe that the energy is deposited more efficiently near the Sun. From determinations of the heating parameter, it was shown that, closer to the Sun, $\gamma \sim 1$, while, closer to the Earth, $\gamma \sim 1.5$. In the Sun, we believe that the dissipation of magnetosonic waves near the base of wind is causing a more efficient heating. However, it is very challenging to implement detailed energetic processes in a $3 \mathrm{D}$ code. We opted to mimic energy deposition by letting $\gamma$ vary radially in the wind. In Chap. 5 we derived the new equation of energy for a variable $\gamma$ and we simulated two new cases: V01 and V02 (Table 5.1). These cases are essentially the same as two particular cases presented in Chap. 4 (namely S01 and S02), except for the radial dependence of $\gamma$ (constant in Chap. 4 versus variable in Chap. 5). We compared these four cases and we showed that the results obtained using the approach of variable $\gamma$ do not considerably differ from the results where $\gamma$ was considered constant, i.e., the steadystate structure of the wind and configuration of the magnetic field are very similar using both approaches. We have to be aware that we have mimicked the energy deposition in 
a smooth way (varying $\gamma$ linearly from 1 to 1.5 in a radial range of $1 r_{0}$ up to 1 AU). We expect that a more dramatic variation of $\gamma$ would cause a more significant difference in the wind structure. We also estimated the X-ray luminosity of the simulations S01, S02, V01, and V02 by assuming that the X-ray originates inside closed magnetic structures. We showed that the four cases simulated present roughly the same X-ray luminosity observed for the Sun and solar-like stars. In face of the result obtained in Chap. 5, we kept on using a heating parameter that is constant along the wind.

In Chap. 6 (Vidotto et al. 2009b) we study magnetized winds of PMS stars. In this evolutionary phase, low-mass stars have more intense magnetic fields and winds, and rotate faster. Hence, we adapted the model described in Chap. 4 to take into account these different characteristics. In the PMS phase, T Tauri stars have accretion disks that tend to dissipate in $\sim 10$ Myr after the stellar birth. As the accretion disk can disturb the coronal magnetic field, we chose to simulate WTTSs, as the presence of the tenuous accretion disk is not expected to strongly influence the coronal structure of the wind and the magnetic field. We simulated seven cases with different stellar periods of rotation, surface magnetic field intensities, surface densities, and $\gamma$ (Table 6.1). We showed once more that the parameter $\beta_{0}$ is very important in the determination of the magnetic configuration of the wind and, if $\beta_{0} \ll 1$, the field presents very elongated structures. By considering our results, we analyzed whether the stellar wind is able to cause planetary migration due to drag forces acting in the azimuthal direction. Our study was motivated by the findings of Lovelace et al. (2008), who showed that planetary migration could occur in relatively short timescales when disturbed by stellar winds described by the Weber-Davis model (§1.3.2). From our model, we obtained larger timescales than the timescales computed by Lovelace et al. (2008) using the Weber-Davis model, which means that planetary migration is not influenced by the stellar winds we simulated. However, these timescales could be reduced if the star had more intense surface magnetic field strengths or higher coronal base densities, as well as if the stellar magnetosphere presented a misalignment angle with the axis of rotation.

In Chap. 7 we presented preliminary results of such configuration. Considering the stellar magnetic moment vector is inclined by an angle $\theta_{t}$ from the axis of rotation of the star, we performed three simulations of WTTS varying the misalignment angle, which was chosen to be $\theta_{t}=10^{\circ}, 20^{\circ}$, and $30^{\circ}$. We showed that the system does not achieve steady state but, instead, presents a periodic behavior with the same period of rotation of the 
star. As in the aligned case, the coronal magnetic field presents zones of open and closed field lines. However, the entire magnetic coronal structure shows a periodic motion. The zone of closed field lines, for example, oscillates around the equatorial plane $(z=0)$ and the amplitude of these oscillations increases for larger $\theta_{t}$. This periodic movement of the magnetosphere affects the entire wind structure. Also, we showed that the wind is more accelerated for larger $\theta_{t}$. A more complete analysis of this configuration will be pursued in the near future, including simulations with a larger range of parameters.

Certainly, there are many other topics that can be investigated using the powerful tool we have described in this thesis, and additional applications of the presented models and other implementations are foreseen.

For example, we have been using a magnetic dipole as initial and boundary conditions of the simulations. However, the use of multi-polar fields could easily be implemented in the numerical code. A more realistic approach would be the inclusion of observed surface magnetograms in the simulations. To our knowledge, there are no simulations with realistic magnetograms for any star other than the Sun. How different would be the structure of the wind if a more realistic magnetogram were used? There are in the literature extrapolations of surface magnetograms with the potential field source surface technique (Jardine et al. 2008, Gregory et al. 2008). In this technique, the wind is not taken into account and the magnetic field is assumed to be force-free, and because of that, it requires less computational resources. A comparison of such technique with the results from our simulations could be very elucidative.

One limitation of our method and of 3D studies in general is that the detailed energetic processes are not considered. Such calculations only exist in 1D studies, as the self-consistent implementation of such processes in 3D codes may be very challenging. Nevertheless, another potential topic of investigation would be to include MHD waves or turbulence in the model, which could eliminate the use of the heating parameter $\gamma$.

An extension of the models described in Chap. 6 would be to consider an accretion disk in the study of magnetized winds of young stars, extending our simulations for WTTSs to CTTSs. This could help us to understand how the interaction of the stellar magnetosphere with the accretion disk takes place, or how the stellar magnetosphere is disturbed due to the presence of the disk, for example.

It would also be interesting to investigate, for instance, the interaction of an extrasolar planet with the stellar wind. To do that, we would have to consider the planet 
in the simulations. Lately, the study of extra-solar planets have been a very popular subject of investigation. As of October 2009, there have been discovered and confirmed about 300 companions to solar-type stars, with masses below 25 Jupiter's mass. Recent space missions are dedicated to exoplanetary research, such as the Corot and the Kepler missions. Thus, theoretical and numerical studies have to be carried out to understand the large amount of information that should outcome from these missions.

Despite the long history of studies of magnetized winds, significant questions about the global structure of stellar coronal winds and their interaction with circumstellar material and planetary systems still remain unanswered. Studies of magnetized coronal winds should benefit from the joint effort between simulationists, theoreticians, and observational astronomers. 


\section{Appendix A}

\section{Refereed Publications}

he refereed publications from which part of this thesis derived are:

1. Vidotto, A. A., Opher, M., Jatenco-Pereira, V. and Gombosi, T. I.: 2009a, Threedimensional Numerical Simulations of Magnetized Winds of Solar-like Stars, ApJ 699,441

2. Vidotto, A. A., Opher, M., Jatenco-Pereira, V. and Gombosi, T. I.: 2009b, Simulations of Winds of Weak-Lined T Tauri Stars: The Magnetic Field Geometry and The Influence of the Wind on Giant Planet Migration, ApJ 703, 1734

As part of the doctoral program, I was also involved in other topics of research, which resulted in the following publications:

3. Vidotto, A. A., Falceta-Gonçalves, D. and Jatenco-Pereira, V.: 2006, A SelfConsistent Determination of the Temperature Profile and The Magnetic Field Geometry in Winds of Late-Type Stars, Space Science Reviews 122, 181

4. Falceta-Gonçalves, D., Vidotto, A. A. and Jatenco-Pereira, V.: 2006, On the Magnetic Structure and Wind Parameter Profiles of Alfvén Wave Driven Winds in Late-Type Supergiant Stars, MNRAS 368, 1145

All these publications can be found in the following pages. 


\title{
THREE-DIMENSIONAL NUMERICAL SIMULATIONS OF MAGNETIZED WINDS OF SOLAR-LIKE STARS
}

\author{
A. A. Vidotto ${ }^{1,2}$, M. Opher $^{2}$, V. Jatenco-Pereira ${ }^{1}$, And T. I. Gombosi ${ }^{3}$ \\ ${ }^{1}$ University of São Paulo, Rua do Matão 1226, São Paulo, SP 05508-090, Brazil; aline@ astro.iag.usp.br \\ ${ }^{2}$ George Mason University, 4400 University Drive, Fairfax, VA 22030-4444, USA \\ ${ }^{3}$ University of Michigan, 1517 Space Research Building, Ann Arbor, MI 48109-2143, USA \\ Received 2008 August 13; accepted 2009 April 29; published 2009 June 11
}

\begin{abstract}
By means of self-consistent three-dimensional magnetohydrodynamics (MHD) numerical simulations, we analyze magnetized solar-like stellar winds and their dependence on the plasma- $\beta$ parameter (the ratio between thermal and magnetic energy densities). This is the first study to perform such analysis solving the fully ideal three-dimensional MHD equations. We adopt in our simulations a heating parameter described by $\gamma$, which is responsible for the thermal acceleration of the wind. We analyze winds with polar magnetic field intensities ranging from 1 to $20 \mathrm{G}$. We show that the wind structure presents characteristics that are similar to the solar coronal wind. The steady-state magnetic field topology for all cases is similar, presenting a configuration of helmet streamer-type, with zones of closed field lines and open field lines coexisting. Higher magnetic field intensities lead to faster and hotter winds. For the maximum magnetic intensity simulated of $20 \mathrm{G}$ and solar coronal base density, the wind velocity reaches values of $\sim 1000 \mathrm{~km} \mathrm{~s}^{-1}$ at $r \sim 20 r_{0}$ and a maximum temperature of $\sim 6 \times 10^{6} \mathrm{~K}$ at $r \sim 6 r_{0}$. The increase of the field intensity generates a larger "dead zone" in the wind, i.e., the closed loops that inhibit matter to escape from latitudes lower than $\sim 45^{\circ}$ extend farther away from the star. The Lorentz force leads naturally to a latitude-dependent wind. We show that by increasing the density and maintaining $B_{0}=20 \mathrm{G}$ the system recover back to slower and cooler winds. For a fixed $\gamma$, we show that the key parameter in determining the wind velocity profile is the $\beta$-parameter at the coronal base. Therefore, there is a group of magnetized flows that would present the same terminal velocity despite its thermal and magnetic energy densities, as long as the plasma- $\beta$ parameter is the same. This degeneracy, however, can be removed if we compare other physical parameters of the wind, such as the mass-loss rate. We analyze the influence of $\gamma$ in our results and we show that it is also important in determining the wind structure.

Key words: methods: numerical - MHD - stars: late-type - stars: winds, outflows
\end{abstract}

Online-only material: color figures

\section{INTRODUCTION}

Studies of the solar corona (SC) have played a crucial role in understanding stellar winds in general. Due to our privileged position immersed in the solar wind, researchers have had access to a great quantity of data that allow a detailed understanding of the physics that is operating in the Sun. Recent sophisticated observations, e.g., via SOHO and Ulysses (McComas et al. 1995; Suess \& Smith 1996; Jones et al. 1998; Wilhelm 2006, among others), have shown that the SC is a highly complex system. It consists of long-lived features, such as the fast and slow solar wind, streamers, and coronal holes, and also of short-lived features, such as the coronal mass ejections, solar flares and sunspots. Hence, if one could separate the long-lived structures from the short-lived ones, one could, in principle, come to a better understanding of the SC itself and other similar outflows. In this paper, we aim to investigate in detail the behavior of the long-lived coronal features in solar-like stellar winds.

Direct measurements of tenuous coronal winds for other stars rather than the Sun have proved to be very difficult to do, although indirect detections of stellar coronal winds have been performed (Wood et al. 2005). As it occurs in the SC, it is very probable that the magnetic field is playing an important role in coronal winds of solar-like stars. Magnetic activity has not only been detected in other stars (Bagnulo et al. 2002; Reiners \& Basri 2006; Phan-Bao et al. 2006; Wade et al. 2007), but also in stars similar to the Sun (Robinson et al. 1980; Petit et al. 2005).
In the absence of a magnetic field, a non-rotating corona expands spherically (Parker 1958; Velli 1994). A different picture, however, is expected if the magnetic energy density at the base of the corona is at least of the same amount of the thermal energy density.

A particular case of equal magnetic and thermal energy densities was explored by Pneuman \& Kopp (1971, hereafter PK71) for the case of the magnetized solar wind. By means of a numerical iterative method, they concluded that the SC is composed of two different magnetic structures in large scale: a region of closed magnetic field loops near the star at low latitudes and at high latitudes, open field lines that cannot restrain the expanding gas.

Astrophysical outflows have long been studied (Parker 1958; Weber \& Davis 1967; Mestel 1968; Nerney \& Suess 1975). Several analytical studies were made toward the understanding of an expanding magnetized corona. For instance, Low \& Tsinganos (1986) found a class of radial analytic solutions for magnetized steady-state winds by removing the latitude dependence of the problem. By varying the global magnetic field geometry and other parameters of their model, such as the velocity at the base of the wind, they analyzed the behavior of flows along open magnetic field lines. Tsinganos \& Trussoni (1991) relaxed the assumption of a polytropic equation of state, but neglected the meridional component of the flow. Sauty \& Tsinganos (1994) found a new class of analytical solutions calculating the exact shape of a field line along the flow rather than assuming a global magnetic field geometry. They showed that for a rotating young stellar object the shape of the field lines 
is an important parameter for the formation of a collimated jet/non-collimated wind. In a more recent work, Lima et al. (2001) constructed exact solutions for a rotating, magnetized wind. They verified that the wind is highly non-spherically symmetric. However, as they pointed out, their model has limitations, as, for instance, meridional flows and the meridional component of the magnetic field were neglected.

Meanwhile, numerical studies were carried out with increasing level of sophistication. Washimi \& Shibata (1993) studied the influence of the stellar rotation on the wind structure and acceleration performing two-dimensional simulations. Solar wind parameters were used, except for the uniform angular velocity at the surface of the star that was varied as to analyze the effects of the centrifugal force. They showed that if the angular velocity is increased more than 10 times the solar value, the centrifugal force becomes comparable to the thermal force, influencing the meridional structure of the wind. Keppens \& Goedbloed (1999) presented detailed one-dimensional and two-dimensional ideal magnetohydrodynamic (MHD) numerical simulations of a polytropic, axisymmetric wind. In a posterior work, Keppens \& Goedbloed (2000) modeled stellar axisymmetric rotating outflows by solving the ideal MHD equations, investigating the effects of open and closed magnetic field lines in the wind. By varying the extension of the closed field line region, as well as the intensity of the magnetic field, they showed that the global wind structure is modified. Ud-Doula (2003), working toward the modeling of hot-star winds, presented a simulation of the solar wind using time-dependent, axisymmetric, MHD simulation. He showed that the MHD modeling was consistent with the work of PK71. Modeling the propagation of a coronal mass ejection from the inner SC to $1 \mathrm{AU}$, Groth et al. (2000) numerically reproduced the steady-state bi-modal nature of the solar wind with a prescribed ad hoc heating mechanism. Working on the modeling of the solar wind, Roussev et al. (2003) simulated the three-dimensional structure of the solar wind under steady-state conditions, using solar magnetogram data as input parameters for the initiation of the wind, and considering a variable heating mechanism. Cohen et al. (2007) extended the Roussev et al. (2003) work considering, as a heating mechanism, a radial dependence of the ratio of specific heats, $\gamma=\gamma(r)$, as to reproduce the observed bi-modality of the velocity of the solar wind.

Despite all the notable evolution of both analytical and numerical studies performed in the last decades, we are far from a satisfactory three-dimensional MHD description of a magnetized wind. Several approximations were made in order to make the system analytically and numerically tractable (e.g., neglecting meridional flows, assuming a polytropic equation of state, assuming a magnetic field topology).

In the present study, we investigate the influence of the magnetic field in solar-like stellar winds with different plasma$\beta$ (the ratio between thermal and magnetic energy densities). We solve the fully three-dimensional MHD equations with the temporal evolution of the energy equation. Therefore, the topology of the field is not restricted and the steady state arises from the dynamical interplay of the outflow and the field. Also, meridional flows arise naturally in the system. We neglect the stellar rotation. The results presented here are thus valid for non-rotators or slow rotators in the region where the toroidal component of the field is still much smaller than the poloidal component.

The paper is organized as follows. In Section 2 we present the numerical scheme used, and in Section 3 the results obtained. Section 4 is dedicated to conclusions and discussion.

\section{THE NUMERICAL MODEL}

To perform the simulations, we make use of the Block Adaptive Tree Solar-wind Roe Upwind Scheme (BATS-RUS), a three-dimensional MHD numerical code developed at the Center for Space Environment Modeling at University of Michigan (Powell et al. 1999).

BATS-R-US uses a computational domain that is blockbased, consisting of Cartesian blocks of cells that can be adaptively refined. It has been widely used to simulate the Earth's magnetosphere (Ridley et al. 2006), the heliosphere (Roussev et al. 2003; Cohen et al. 2007), the outerheliosphere (Linde et al. 1998; Opher et al. 2003, 2004), coronal mass ejections (Manchester et al. 2004; Lugaz et al. 2005), and the magnetosphere of planets (Tóth et al. 2004; Hansen et al. 2005), among others. In this work, we adapted the version for the outer heliosphere (Opher et al. 2003, 2004) to study the problem of the wind of a solar-like star.

The code solves the ideal MHD equations, that in the conservative form are given by

$$
\begin{gathered}
\frac{\partial \rho}{\partial t}+\nabla \cdot(\rho \mathbf{u})=0 \\
\frac{\partial(\rho \mathbf{u})}{\partial t}+\nabla \cdot\left[\rho \mathbf{u} \mathbf{u}+\left(p+\frac{B^{2}}{8 \pi}\right) I-\frac{\mathbf{B} \mathbf{B}}{4 \pi}\right]=\rho \mathbf{g} \\
\frac{\partial \mathbf{B}}{\partial t}+\nabla \cdot(\mathbf{u} \mathbf{B}-\mathbf{B} \mathbf{u})=0 \\
\frac{\partial \varepsilon}{\partial t}+\nabla \cdot\left[\mathbf{u}\left(\varepsilon+p+\frac{B^{2}}{8 \pi}\right)-\frac{(\mathbf{u} \cdot \mathbf{B}) \mathbf{B}}{4 \pi}\right]=\rho \mathbf{g} \cdot \mathbf{u},
\end{gathered}
$$

where $\rho$ is the mass density, $\mathbf{u}$ the plasma velocity, $\mathbf{B}$ the magnetic field, $p$ the gas pressure, $\mathbf{g}$ the gravitational acceleration due to the central body, and $\varepsilon$ is the total energy density given by

$$
\varepsilon=\frac{\rho u^{2}}{2}+\frac{p}{\gamma-1}+\frac{B^{2}}{8 \pi} .
$$

We consider ideal gas, so $p=\rho k_{B} T /\left(\mu m_{p}\right)$, where $k_{B}$ is the Boltzmann constant, $T$ the temperature, $\mu m_{p}$ the mean mass of the particle, and $\gamma$ is the ratio of the specific heats.

Due to the lack of knowledge of all the detailed processes that take place in a stellar wind, it is difficult to estimate all the mechanisms that modify the heat content of the wind (e.g., conduction, radiation, mechanical dissipation of energy that is transferred to the plasma). MHD waves and turbulence are known to play an important role on solar wind acceleration and heating (Matthaeus et al. 1999; Cranmer \& van Ballegooijen 2005; Cranmer et al. 2007). For this reason, they are often included in stellar wind models as well (e.g., Airapetian et al. 2000; Vidotto \& Jatenco-Pereira 2006; Suzuki 2007). The solarstellar wind connection is usually made by scaling the observed solar wind characteristics (e.g., the wave flux and spectral slope, solar magnetograms) to other stars (Jatenco-Pereira \& Opher 1989; Schrijver 2001). However, it is not clear how these characteristics scale to other solar-like stars, in this first analysis we start with a simpler treatment of the mechanisms of acceleration of the wind. We adopt, therefore, an approach similar to Roussev et al. (2003) who considered that $\gamma$ is associated with "turbulent" internal degrees of freedom, in a way analogous to the Sun, where a significant amount of energy is stored in the form of waves and turbulent fluctuations. 

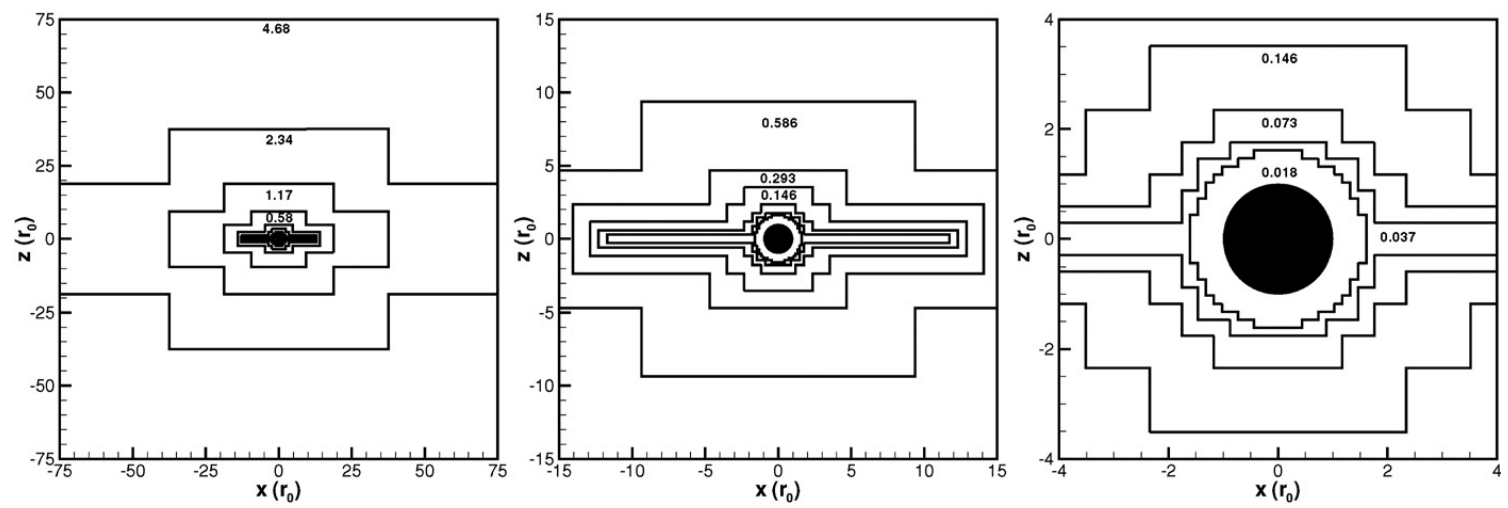

Figure 1. Three different views of the grid. Inside each region, we indicate the maximum cell size of the region (in $r_{0}$ units).

\subsection{The Grid Adopted}

For all the simulations done, we adopted the same grid resolution. Initially, the simulation domain is refined in five levels. Other five refinement levels that are body-focused and focused on the equatorial plane (current sheet region) are applied next. Finally, an additional level is applied to the body. There are $9.1 \times 10^{6}$ cells in the domain. The smallest cell size is $0.018 r_{0}$, located around the central body. The maximum cell size is $4.68 r_{0}$. The cell size near the current sheet is $0.036 r_{0}$. The grid is Cartesian $\{x, y, z\}$ and the center of the star is placed at the origin. The axes $x, y$, and $z$ extend from $-75 r_{0}$ to $75 r_{0}$. The grid can be seen in Figure 1.

\subsection{The Initial Conditions}

The simulation domain is initialized with a solar-like star at its center with $1 M_{\odot}$ and $r_{0}=1 R_{\odot}$. The grid is initialized with a one-dimensional hydrodynamical (HD) wind for a totally ionized plasma of hydrogen. The initial temperature profile is set according to the value of $\gamma$ adopted. This solution is dependent solely on the choice of the base temperature of the wind and the only physical possible solution is the one that becomes supersonic when passing through the critical radius (Parker 1958). Due to conservation of mass of a steady wind, we obtain the density profile from the radial velocity profile $u_{r}(r)$. Initially, we chose a coronal base temperature of $T_{0}=1.56 \times 10^{6} \mathrm{~K}$ and a base density of $\rho_{0}=1.544 \times 10^{-16} \mathrm{~g} \mathrm{~cm}^{-3}$ (we vary $\rho_{0}$ in the second and third sets of simulations presented later on).

The simulations are initialized with a bipolar magnetic field configuration described in spherical coordinates $\{r, \theta, \varphi\}$ by

$$
\mathbf{B}=\frac{B_{0} r_{0}^{3}}{r^{3}}\left(\cos \theta, \frac{1}{2} \sin \theta, 0\right),
$$

where $B_{0}$ is the magnetic field intensity at the poles, $r$ the radial coordinate, $\theta$ the co-latitude, and $\varphi$ is the azimuthal angle measured in the equatorial plane. The system is then evolved in time until steady state is achieved.

\subsection{The Boundary Conditions}

The inner boundary of the system is considered to be the base of the wind at $r=r_{0}$ and its conditions are dependent on local flow conditions: plasma can freely leave the reservoir (i.e., the base of the coronal wind), but no "backflow" is allowed. Fixed boundary conditions were adopted at $r=r_{0}$. The outer
Table 1

The Complete Set of Simulations

\begin{tabular}{lccccc}
\hline \hline Name & $T_{0}(\mathrm{MK})$ & $\rho_{0} / \rho_{c}{ }^{\mathrm{a}}$ & $B_{0}(\mathrm{G})$ & $\beta_{0}$ & $\gamma$ \\
\hline S00 & 1.56 & 1 & 0 & $\infty$ & 1.01 \\
\hline \multicolumn{5}{c}{ First Set of Simulations } \\
\hline S01 & 1.56 & 1 & 1 & 1 & 1.01 \\
S02 & 1.56 & 1 & 5 & $1 / 25$ & 1.01 \\
S03 & 1.56 & 1 & 10 & $1 / 100$ & 1.01 \\
S04 & 1.56 & 1 & 15 & $1 / 225$ & 1.01 \\
S05 & 1.56 & 1 & 20 & $1 / 400$ & 1.01 \\
\hline \multicolumn{5}{c}{ Second Set of Simulations } \\
\hline S06 & 1.56 & 1.8 & 20 & $1 / 225$ & 1.01 \\
S07 & 1.56 & 4 & 20 & $1 / 100$ & 1.01 \\
S08 & 1.56 & 16 & 20 & $1 / 25$ & 1.01 \\
S09 & 1.56 & 400 & 20 & 1 & 1.01 \\
\hline \multicolumn{5}{c}{ Third Set of Simulations } \\
\hline S01b & 1.56 & 1 & 1 & 1 & 1.1 \\
S02b & 1.56 & 1 & 5 & $1 / 25$ & 1.1 \\
S08c & 1.56 & 16 & 20 & $1 / 25$ & 1.1 \\
S09c & 1.56 & 400 & 20 & 1 & 1.1 \\
\hline
\end{tabular}

Note. ${ }^{\mathrm{a}} \rho_{c}=1.544 \times 10^{-16} \mathrm{~g} \mathrm{~cm}^{-3}$

boundary has outflow conditions, i.e., a zero gradient is set to all the primary variables.

\section{RESULTS}

Table 1 presents the parameters used for the simulations performed. S00 is a purely HD simulation included for comparison purposes. The simulations are divided in three sets. The first set of simulations, composed by simulations S01 to S05, aims to investigate the effect of the magnetic energy density on the wind. We increased $B_{0}$ from 1 to $20 \mathrm{G}$ while maintaining the other initial values fixed: $\beta_{0}$, the ratio between the surface thermal pressure $p_{0}$ and the magnetic pressure evaluated at the pole

$$
\beta_{0}=\frac{8 \pi p_{0}}{B_{0}^{2}},
$$

is decreased from 1 to $1 / 400$. S01 is similar to the case studied by PK71 with $\beta_{0}=1$. The initial HD wind is the same for all the simulations of the first set $\left(\rho_{0}=\rho_{c}=1.544 \times 10^{-16} \mathrm{~g}\right.$ $\mathrm{cm}^{-3}, T_{0}=1.56 \times 10^{6} \mathrm{~K}$, as can be seen in Table 1). In the second set of simulations, from simulations S06 to S09, $B_{0}$ is 


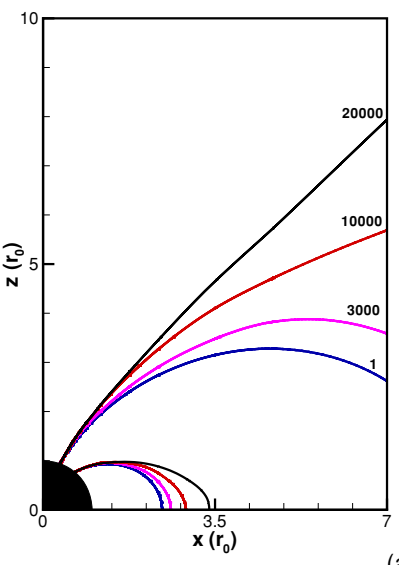

(a)

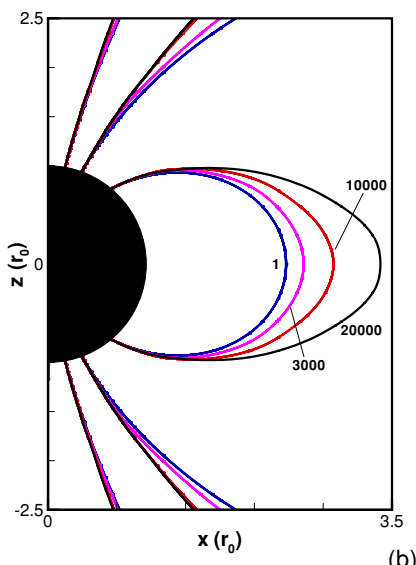

(b)

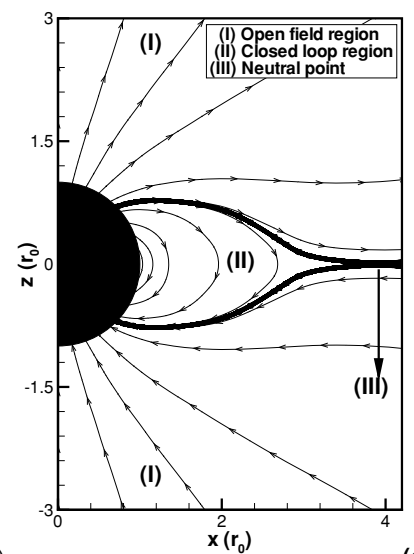

(c)

Figure 2. (a) and (b): evolution of the magnetic field lines for S03. The color of each streamline represents a given iteration, ranging from $N=1(t=0$, blue) to $20,000(t=7 \mathrm{hr}$, black). At $N=1$, the field is a dipole. The steady state is achieved at $N=148,000(t=51 \mathrm{hr})$. (c): Final steady-state configuration of the magnetic lines: (1) represents the zone of open field lines; (2) the zone of closed field lines; (3) the location of the neutral point. The thick line represents the position of the current sheet formed.

(A color version of this figure is available in the online journal.)

fixed at $20 \mathrm{G}$ and we vary $\rho_{0}$ from 1 to 400 times the solar density, $\rho_{c}=1.544 \times 10^{-16} \mathrm{~g} \mathrm{~cm}^{-3}$, thus increasing $\beta_{0}$ back to 1 in S09. In both sets of simulations, we adopted the value $\gamma=1.01$. We then consider a third set of simulations, where parameters similar to the first and second sets are used, but we adopt $\gamma=1.1$.

\subsection{The First Set of Simulations}

\subsubsection{Evolution to Steady State}

The Lorentz force $\mathbf{F}_{\mathbf{B}}$ acting on the flow is given by the difference between the magnetic tension, $\mathcal{T}_{\mathbf{B}}$, and a non-isotropic gradient of the magnetic pressure $P_{B}$

$$
\mathbf{F}_{\mathbf{B}}=\mathcal{T}_{\mathbf{B}}-\nabla_{\perp} P_{B},
$$

where $\nabla_{\perp}$ is the component of the gradient perpendicular to the magnetic field B. As the magnetic field lines are more curved at low latitudes, the tension becomes more important as one approaches the equatorial plane. Hence, opening a bipolar field structure at low latitudes, where lines are more curved, is more difficult than opening a bipolar field topology at high latitudes. As both $\mathcal{T}_{\mathbf{B}}$ and $\nabla_{\perp} P_{B}$ are latitude-dependent, so is $\mathbf{F}_{\mathbf{B}}$. We therefore expect from the interaction of the outflow and the magnetic field a latitude-dependent wind.

Figures 2(a) and (b) present the temporal evolution of the magnetic field lines from iteration $N=1$ to $N=20,000$. This evolution was common to all runs. The plot presented is in the meridional plane, for S03. Figure 2(a) shows that at high latitudes field lines tend to become open. Figure 2(b) shows the initial stretching of the lines emerging from low latitudes along the equator.

As the simulation evolves in time, the magnetic field lines reconnect in the equatorial plane. The steady-state configuration (Figure 2(c)) consists of a formation of a bi-component wind. This configuration is composed by a zone of open magnetic field lines (1) coexisting with a zone of closed loops anchored on the star (2), also called helmet streamer. At the top of the closed loops lies the neutral point of cusp-type (3): approaching the neutral point from inside the closed loops, the magnetic field

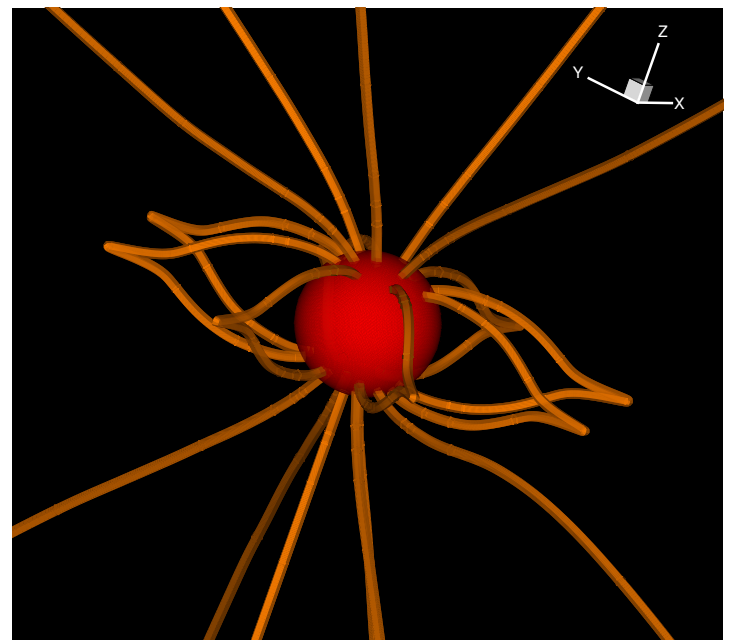

Figure 3. Three-dimensional view of the steady state for S03.

(A color version of this figure is available in the online journal.)

goes to zero. Beyond the neutral point, along the equatorial plane, a current sheet is formed (thick line in Figure 2(c)). The zone of closed field lines is located at low latitudes from $\sim-45^{\circ}$ to $45^{\circ}$ and extends up to the neutral point. Beyond the neutral point, the zone of open field lines fills all the volume. Figure 3 shows a three-dimensional view of the final configuration.

\subsubsection{Steady-State Wind Profile}

Figure 4 shows the total velocity of the wind in the meridional plane for the first set of simulations. The magnetic field lines are the black lines and the Alfvén surface is indicated by the white line. It can be seen that the increase of $B_{0}$ leads to faster winds.

The five panels present similar structures but quantitatively very different. The wind is not spherical; higher velocities are achieved at high latitudes. The higher $B_{0}$ is, the higher is the 

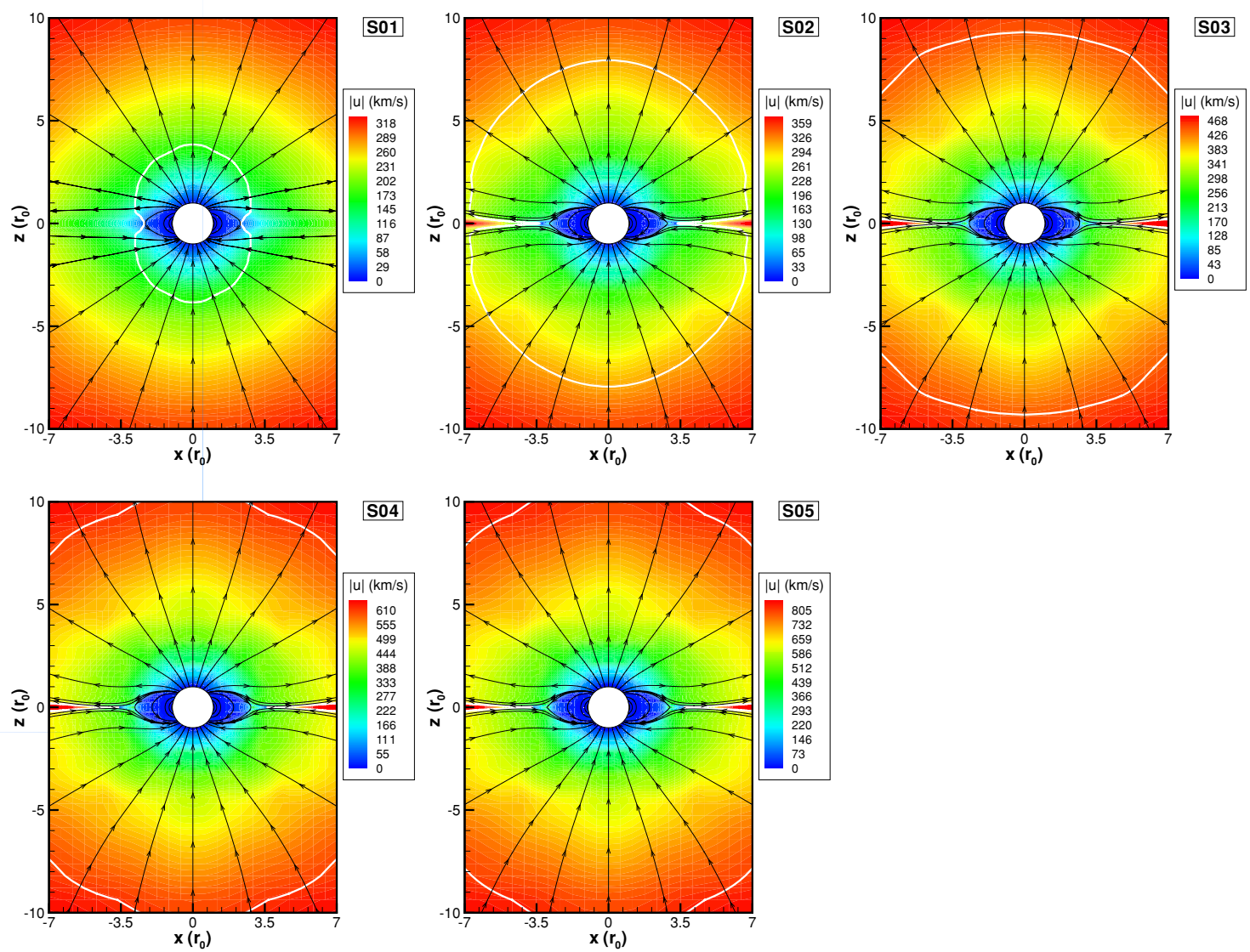

Figure 4. Meridional cuts of the steady-state configurations for simulations S01 to S05. Black streamlines represent the final configuration of the magnetic field. Contour plots of the velocity of the flow are shown in the background. The white line is the Alfvén surface.

(A color version of this figure is available in the online journal.)

departure from spherical symmetry and the higher is the total velocity of the wind.

The bi-modality of the wind is due to the nature of the magnetic force. A purely HD (non-rotating) wind is spherically symmetric but in the MHD case, this symmetry is lost because the magnetic force has a meridional component.

The solar wind has an observed bi-modal velocity ranging from 400 to $800 \mathrm{~km} \mathrm{~s}^{-1}$ at $1 \mathrm{AU}$ (solar minima). Although our model does not make use of magnetograms and thermal latitudinal heating as more realistic solar models such as Cohen et al. (2007) and Liu et al. (2008) do, it is interesting to compare S01 to their results. In the Liu et al. (2008) model, at $r=4.5 r_{0}$, the highest velocity of $\sim 440 \mathrm{~km} \mathrm{~s}^{-1}$ is achieved at the polar axis $\left(\theta=0^{\circ}\right)$, while in the mid-latitudes $\left(\theta \sim 45^{\circ}\right)$, this value is decreased to $\sim 230 \mathrm{~km} \mathrm{~s}^{-1}$. For $\mathrm{S} 01$, the same positions lead to $201 \mathrm{~km} \mathrm{~s}^{-1}$ and $190 \mathrm{~km} \mathrm{~s}^{-1}$, respectively. In our models, we are not treating the presence of waves and damping that is responsible for the latitude dependence that is observed in the solar wind. However, it is interesting that, just by the presence of magnetic field with an initially spherical wind, we obtain a latitude-dependent wind, although not as dramatic as in the Sun.

Figure 5 presents a radial cut at $r=4.5 r_{0}$ for cases $\mathrm{S} 00$ and S01. There is a range of angles, $\sim 30^{\circ}$ above and below
Table 2

Co-Latitude Dependence of $u_{r}$ at $r=4.5 r_{0}$

\begin{tabular}{lccc}
\hline \hline Name & $u_{r}\left(\theta=18^{\circ}\right)$ & $u_{r}\left(\theta=45^{\circ}\right)$ & $\Delta u_{r}{ }^{\mathrm{a}}$ \\
\hline S01 & 200 & 189 & 11 \\
S02 & 243 & 224 & 19 \\
S03 & 329 & 295 & 34 \\
S04 & 456 & 400 & 56 \\
S05 & 610 & 528 & 82 \\
\hline
\end{tabular}

Note. ${ }^{\text {a }} \Delta u_{r}=u_{r}\left(\theta=18^{\circ}\right)-u_{r}\left(\theta=45^{\circ}\right)$.

the equator, where the MHD wind is slower compared to the HD model (SO0). This deceleration is a consequence of the magnetic tension that is stronger near the equator. At high latitudes, the gradient of both thermal and magnetic pressures is responsible for driving the wind. Figure 5 also presents the analytical result for a non-magnetized wind (dashed line) for comparison purposes. The width of the decrease seen in Figure 5 for the S01 curve is dependent on the grid resolution (Opher et al. 2004).

Figure 6 presents the radial cuts for the remaining simulations of the first set from $\theta=0^{\circ}$ to $90^{\circ}$. All the curves present smaller velocities at the equator $\left(\theta=90^{\circ}\right)$ and higher velocities at high 


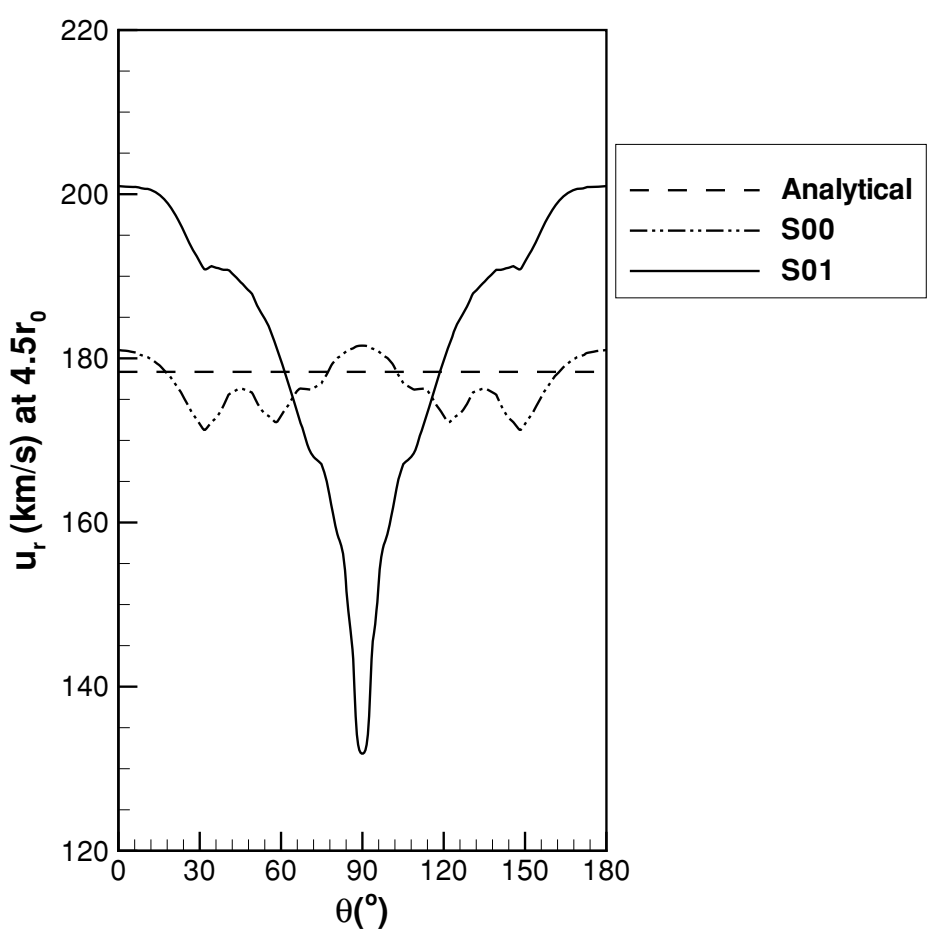

Figure 5. Radial velocity of the wind as a function of $\theta$ at $r=4.5 r_{0}$, for S00 (double-dot-dashed line), S01 (solid line) and analytical solution of a non-magnetized wind (dashed line).

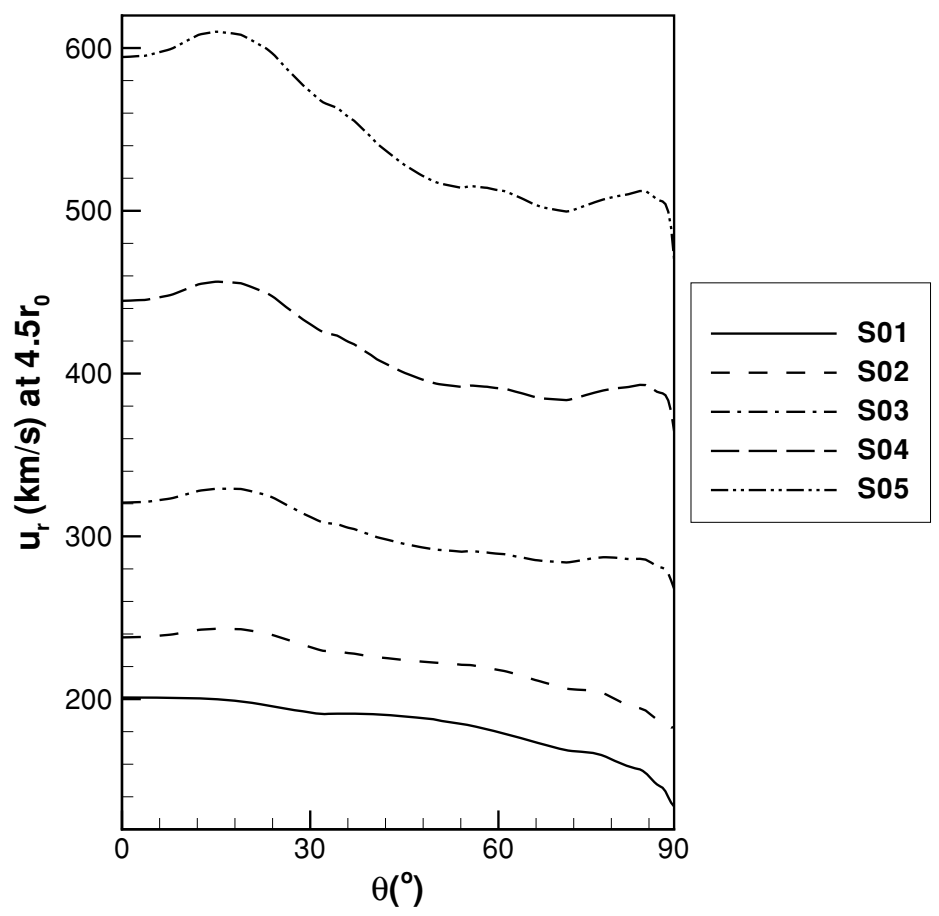

Figure 6. Same as Figure 5, but for S01 to S05, from $\theta=0^{\circ}$ to $\theta=90^{\circ}$. 
Table 3

Mass-loss Rate per Unit Solid Angle of the Outflow ${ }^{\mathrm{a}}$

\begin{tabular}{lcc}
\hline \hline Name & $\dot{m}_{15}\left(\theta=0^{\circ}\right)$ & $\dot{m}_{15}\left(\theta=45^{\circ}\right)$ \\
\hline S02 & 5.1 & 5.7 \\
S03 & 6.0 & 6.6 \\
S04 & 7.1 & 7.5 \\
S05 & 8.2 & 8.7 \\
\hline
\end{tabular}

Note. ${ }^{\mathrm{a}} \dot{m}_{15}(\theta)=\left[\dot{m}(\theta) / 10^{-15}\right] M_{\odot} \mathrm{yr}^{-1}$.

latitudes. The difference in velocities increases as $B_{0}$ is increased (see Table 2).

Another consequence of the latitudinal dependence of the Lorentz force, the mass-loss rate per unit solid angle of a steady wind

$$
\dot{m}(\theta)=r^{2} \rho(\theta) u_{r}(\theta)
$$

is also latitude-dependent. According to Equation (1), $\dot{m}(\theta)$ is constant if there is no variation in $\theta$. In our simulations, this is verified far from the central star. Table 3 presents the calculated $\dot{m}(\theta)$ for two given co-latitudes of the steady-state wind, when it has already reached constant values. $\dot{m}(\theta)$ at the pole is smaller compared to other angles, despite the increase of the radial velocity. The lower value of $\dot{m}(\theta)$ at the pole is due to matter carried from high latitudes to low latitudes (Section 3.1.3). As the latitudinal increase of density is higher than the corresponding decrease in the radial velocity, $\dot{m}(\theta)$ is higher at low latitudes.

\subsubsection{Meridional Flows}

The component of the magnetic force in the $\theta$ direction gives rise to meridional flows, bringing matter from both hemispheres toward the equator. This causes a density enhancement along the equatorial plane.

Figure 7 presents the meridional velocity, $u_{\theta}$, for S01 to S05 at steady state. $u_{\theta}$ increases with $B_{0}$, ranging from a maximum value of $28 \mathrm{~km} \mathrm{~s}^{-1}$ in S01 $\left(B_{0}=1 \mathrm{G}\right)$ to $339 \mathrm{~km} \mathrm{~s}^{-1}$ in S05 $\left(B_{0}=20 \mathrm{G}\right)$. The last panel of Figure 7 presents the flux of matter in the $\theta$ direction, $\rho u_{\theta}$, at $4.5 r_{0}$. It can be seen that as the magnetic field increases there is an increase of the meridional flux of matter.

\subsubsection{The Neutral Point}

The meridional flows are also responsible for compressing lines with opposite polarities along the equatorial plane and due to reconnection, a current sheet is formed. In our simulations, the reconnection is controlled due to numerical resistivity. In order to minimize the effects of numerical resistivity, we refined the grid along the equatorial plane. Ultimately, a more realistic reconnection should be included in the global three-dimensional MHD simulations such as done by Kuznetsova et al. (2007). The reconnection leads to the emergence of a faster wind in the equatorial plane, beyond the reconnection point (Figure 4). Such wind is an artifact of the model and the same behavior was also observed by Roussev et al. (2003).

The location of the neutral point $r_{N}$ is given by the requirement that the total pressure (magnetic plus thermal) at the tip of the cusp of closed loops is continuous

$$
p\left(r_{N}^{+}\right)-p\left(r_{N}^{-}\right)=\frac{B\left(r_{N}\right)^{2}}{8 \pi},
$$

where $p\left(r_{N}^{+}\right)$is the thermal pressure evaluated immediately after $r_{N}$ and $p\left(r_{N}^{-}\right)$immediately before $r_{N}(\mathrm{PK} 71)$. Along a magnetic
Table 4

Location of the Neutral Point, $r_{N}$, Along the Equatorial Plane as a Function of $B_{0}$

\begin{tabular}{lcc}
\hline \hline Name & $B_{0}(\mathrm{G})$ & $r_{N}\left(r_{0}\right)$ \\
\hline S02 & 5 & 3.68 \\
S03 & 10 & 3.90 \\
S04 & 15 & 4.01 \\
S05 & 20 & 4.12 \\
\hline
\end{tabular}

streamline, the magnetic force is null. Assuming an isothermal atmosphere, the energy equation reduces to

$$
\frac{u^{2}}{2}+c_{s}^{2} \ln \frac{p}{p_{0}}+g r=\frac{u_{0}^{2}}{2}+g_{0} r_{0},
$$

where $c_{s}$ denotes the isothermal sound speed and the use of the subscript " 0 " indicates that the quantity is evaluated at the base of the wind. Equation (10) can be written as

$$
\begin{aligned}
p\left(r_{N}^{+}\right)-p\left(r_{N}^{-}\right) & \simeq p\left(r_{N}^{+}\right)\left[\exp \frac{1}{2}\left(\frac{u\left(r_{N}\right)}{c_{s}}\right)^{2}-1\right] \\
& \simeq p\left(r_{N}^{+}\right) \frac{u\left(r_{N}\right)^{2}}{2 c_{s}^{2}}
\end{aligned}
$$

where we neglected the initial velocity of the flow in comparison with the velocity of the flow at the neutral point, $u\left(r_{N}\right)$, and assumed $u\left(r_{N}\right) \ll c_{s}$. Under these considerations, Equations (10) and (12) lead to

$$
\frac{B\left(r_{N}\right)^{2}}{8 \pi} \simeq p\left(r_{N}^{+}\right) \frac{u\left(r_{N}\right)^{2}}{2 c_{s}^{2}} \simeq \frac{1}{2} \rho\left(r_{N}^{+}\right) u\left(r_{N}\right)^{2},
$$

implying that at the neutral point

$$
u\left(r_{N}\right) \simeq v_{A}\left(r_{N}\right),
$$

where $v_{A}$ is the Alfvén velocity, i.e., the neutral point lies on the Alfvén surface.

Figure 7 presents the Alfvén surface (white line), where it can be seen that the above result is confirmed by our simulations. In Table 4 , the position of the neutral point $r_{N}$ is given for each of the simulations of the first set. The cell size at the current sheet is $0.036 r_{0}$, so the numerical error associated with $r_{N}$ is $\pm 0.018 r_{0}$. $r_{N}$ increases as $B_{0}$ is increased, i.e., the "dead-zone" (zone of closed loops) is larger for larger $B_{0}$. As $B_{0}$ is increased from $5 \mathrm{G}$ to $20 \mathrm{G}, r_{N}$ moves from $3.68 r_{0}$ to $4.12 r_{0}$. This is expected, since the Alfvén surface moves farther from the star as $B_{0}$ is increased. S01 does not present reconnection.

Inside the closed field line region, particles are trapped. This is evidenced in Figure 8, where we plot the magnetic field lines and the vectors of the flow velocity. As can be seen, if a given particle emerges inside the closed loop zone, it will remain there, because its velocity is not high enough to escape the effective potential well created by the magnetic tension and gravitational attraction force.

\subsubsection{Energetics}

We would like to estimate the contribution of the different energies to the total energy content of the system.

The temperature $T$ of the steady-state coronal wind increases with $B_{0}$. Figure 9 presents the temperature profiles along the 

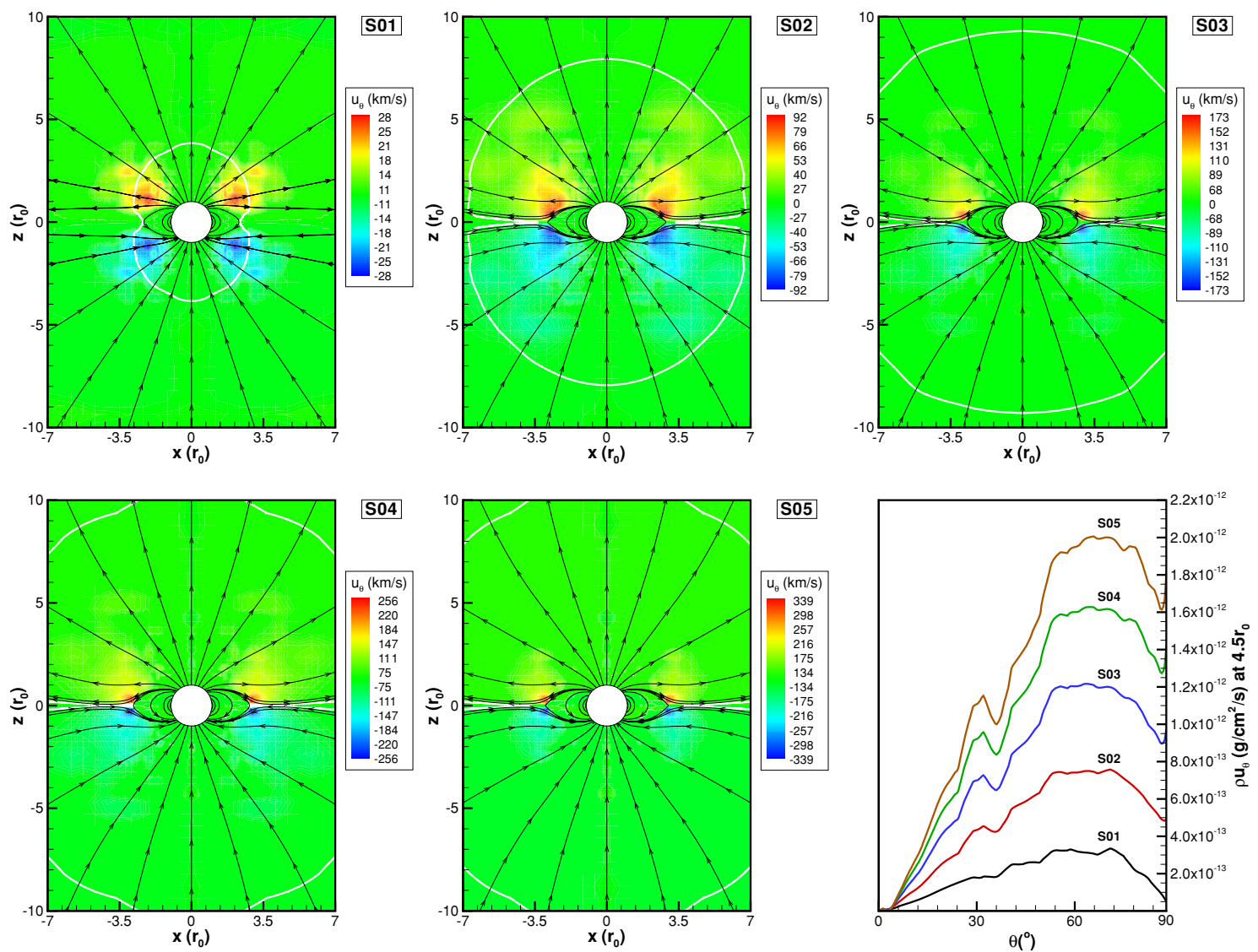

Figure 7. Meridional velocities $u_{\theta}$ for the steady-state configurations of simulations S01 to S05. Black streamlines are the magnetic field lines. The white line is the Alfvén surface. Last panel: the flux of matter in the $\theta$ direction, $\rho u_{\theta}$, for S01 to S05 at $r=4.5 r_{0}$.

(A color version of this figure is available in the online journal.)

polar axis for the cases of the first set of simulations (in steady state). For higher $B_{0}, T$ increases near the base of the wind and it drops slowly for larger radii. For S05, the increase in the temperature is maximum among all the cases, reaching $\simeq 5.9 \mathrm{MK}$ around $6 r_{0}$. In the solar wind, however, the temperature profile is not flat (Matthaeus et al. 2006), because the bulk of the internal energy deposition occurs near the Sun.

For steady state, conservation of total energy flux is given by

$$
\nabla \cdot \mathbf{q}=0
$$

where $\mathbf{q}$ is the total energy flux given by

$$
\mathbf{q}=\mathbf{u}\left(\frac{\rho u^{2}}{2}+\frac{\gamma p}{\gamma-1}+\rho \Phi\right)+\mathbf{S}
$$

and $\Phi$ is the gravitational potential. The terms on the right-hand side of Equation (16) are the kinetic, enthalpy, and gravitational energy fluxes, and $\mathbf{S}$ is the Poynting flux vector that in ideal MHD is given by

$$
\mathbf{S}=\frac{B^{2}}{4 \pi} \mathbf{u}-\frac{(\mathbf{u} \cdot \mathbf{B})}{4 \pi} \mathbf{B} .
$$

Taking a volume defined by a given magnetic flux tube bounded by two cross-sections (see Figure 10), the net energy flux q should be conserved.

Table 5 presents each parcel of the total energy flux that crosses four cross-sectional areas of a magnetic flux tube whose central axis is located at $\theta \sim 30^{\circ}$. At $2.2 r_{0}$, the enthalpy power (i.e., the thermal plus internal energy fluxes integrated over the area of the cross-section of the tube at this position) is already the dominant form of energy. At 3.0 $r_{0}$, magnetic power is almost negligible indicating that the continuous acceleration of the flow from there on is driven by solely the enthalpy. At $r=4.2 r_{0}$ and $6.0 r_{0}$, the conversion of enthalpy into kinetic energy is responsible for the continuous acceleration of the wind until a terminal velocity is reached.

\subsection{The Second Set of Simulations}

The second set of simulations aims to investigate the effect of the density on the wind. We vary the initial density maintaining the same surface magnetic field $B_{0}$ and surface temperature $T_{0}$ adopted in $\mathrm{S} 05$. The simulations we analyze in this section consist of simulations S06 to S09 (Table 1).

We note that as $\rho_{0}$ increases, there is a decrease in the wind velocity, as can be seen in Figure 11, where we plotted the radial 


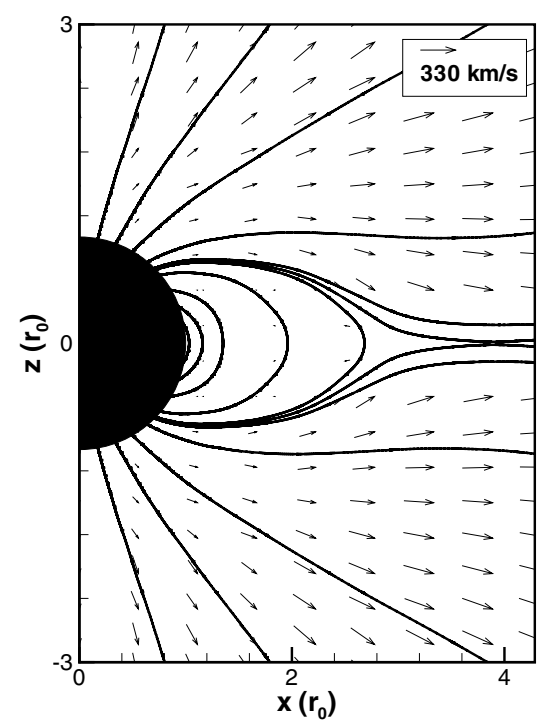

Figure 8. Trapping of particles inside the closed loop region for S03. Thick lines represent the magnetic field lines and vectors represent the flow velocity, both in the meridional plane.

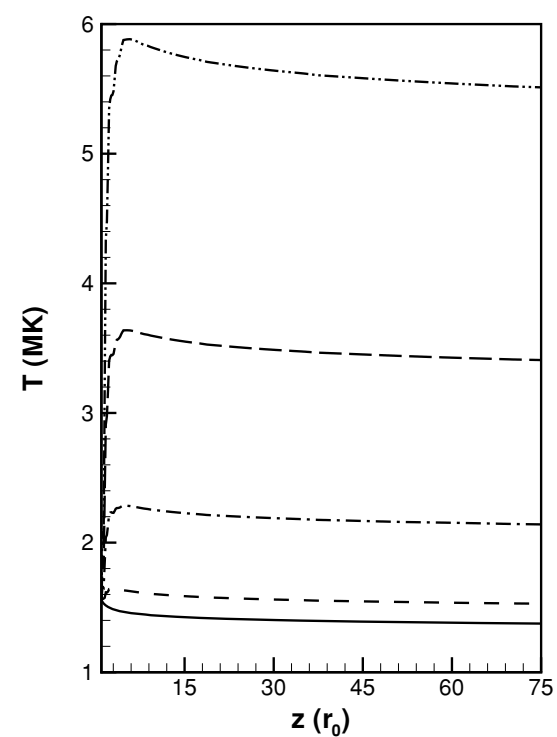

Figure 9. Temperature profile along the polar axis.

velocity of the flows along $\theta=45^{\circ}$. Also, as $\rho_{0}$ is increased, the reconnection point moves closer to the star.

\subsection{Comparison Between First and Second Sets}

Comparing the first and the second set of simulations, we note that flows with same $\beta_{0}$ possess the same wind velocity profiles (S01/S09, S02/S08, S03/S07, and S04/S06). This result can be verified from Equations (1) to (4). The magnetic field can be written in terms of a normalized field $\tilde{B}$ with a normalization constant $B_{0}$

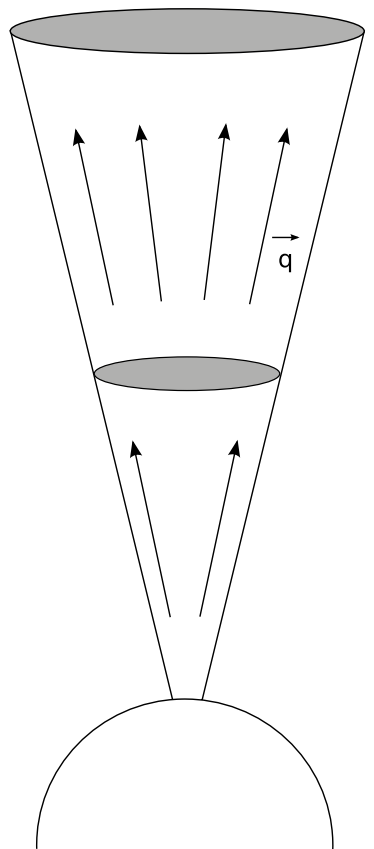

Figure 10. Cartoon of the energy flux $q$ along a flux tube.

Table 5

Percentage Amount of Energy Flux Passing Through Four Different Cross-Sections Along a Flux Tube for S03 and S05

\begin{tabular}{lcccc}
\hline \hline Energy Flux & $2.2 r_{0}$ & $3 r_{0}$ & $4.2 r_{0}$ & $6 r_{0}$ \\
\hline & \multicolumn{5}{c}{$\mathrm{S} 03$} \\
\hline Magnetic & 0.15 & 0.07 & 0.02 & 0.01 \\
Kinetic & 0.32 & 0.59 & 1.26 & 1.76 \\
Enthalpy & 97.08 & 97.63 & 97.50 & 97.39 \\
Grav. & 2.45 & 1.72 & 1.22 & 0.85 \\
\hline & \multicolumn{5}{c}{$\mathrm{S} 05$} \\
\hline Magnetic & 0.41 & 0.15 & 0.02 & 0.02 \\
Kinetic & 0.46 & 0.85 & 1.77 & 2.33 \\
Enthalpy & 98.04 & 98.27 & 97.72 & 97.31 \\
Grav. & 1.09 & 0.72 & 0.50 & 0.34 \\
& & & &
\end{tabular}

$$
\mathbf{B}=\tilde{\mathbf{B}} B_{0} .
$$

Doing the same for the density

$$
\rho=\tilde{\rho} \rho_{0} .
$$

In this case, Equations (1) to (4) are written as

$$
\begin{gathered}
\frac{\partial \tilde{\rho}}{\partial t}+\nabla \cdot(\tilde{\rho} \mathbf{u})=0 \\
\frac{\partial(\tilde{\rho} \mathbf{u})}{\partial t}+\nabla \cdot\left[\begin{array}{c}
\left.\tilde{\rho} \mathbf{u}+\left(\tilde{p}+\frac{B_{0}^{2}}{\rho_{0}} \frac{\tilde{B}^{2}}{8 \pi}\right) I-\frac{B_{0}^{2}}{\rho_{0}} \frac{\tilde{\mathbf{B}}}{4 \pi}\right]=\tilde{\rho} \mathbf{g} \\
\frac{\partial \tilde{\mathbf{B}}}{\partial t}+\nabla \cdot(\mathbf{u} \mathbf{\mathbf { B }}-\tilde{\mathbf{B}} \mathbf{u})=0 \\
\frac{\partial \tilde{\varepsilon}}{\partial t}+\nabla \cdot\left[\mathbf{u}\left(\tilde{\varepsilon}+\tilde{p}+\frac{B_{0}^{2}}{\rho_{0}} \frac{\tilde{B}^{2}}{8 \pi}\right)-\frac{B_{0}^{2}}{\rho_{0}} \frac{(\mathbf{u} \cdot \tilde{\mathbf{B}})}{4 \pi}\right]=\tilde{\mathbf{B}}
\end{array}\right]=\tilde{g} \cdot \mathbf{u},
\end{gathered}
$$




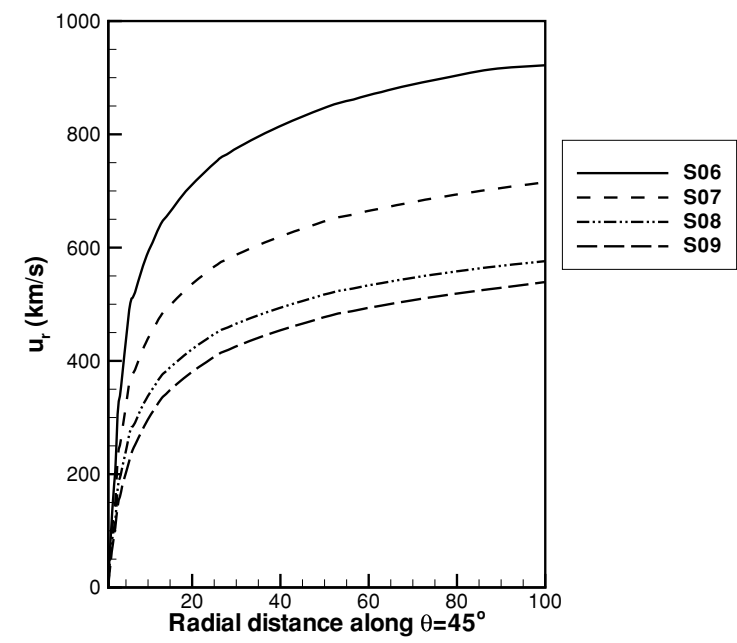

Figure 11. Radial velocity profiles as a function of the distance along $\theta=45^{\circ}$ for the second set of simulations.

where

$$
\tilde{\varepsilon}=\frac{\tilde{\rho} u^{2}}{2}+\frac{\tilde{p}}{\gamma-1}+\frac{B_{0}^{2}}{\rho_{0}} \frac{\tilde{B}^{2}}{8 \pi} .
$$

Equations (18) and (20) show that the normalized density profile $(\tilde{\rho})$ and the normalized field profile $(\tilde{B})$ are independent of the values $\rho_{0}$ and $B_{0}$ adopted at the surface. Equations (19) and (21) show that the choice of $\rho_{0}$ and $B_{0}$ (or in other words the choice of $\beta_{0} \propto \rho_{0} / B_{0}^{2}$, as we assumed the same base temperature) appears explicitly in the momentum and energy equations. According to Equations (18)-(21), it is expected that flows with same $\beta_{0}$ have the same wind velocity, magnetic field configuration, density profile, etc. Although the normalized density and magnetic field profiles are the same if $\beta_{0}$ is the same in two different simulations, the mass-loss rates are different, where the value of the normalization constant $\rho_{0}$ has to be considered.

Hence, by analyzing only the velocity of an outflow, one cannot equivocally predict its physical characteristics at its base. Our results indicate that there is a group of magnetized flows that would present the same terminal velocity despite its thermal and magnetic energy densities, as long as $\beta_{0}$ is the same.

This degeneracy can be removed if we consider the massloss rate. As the velocity profiles for two different magnetized outflows with similar $\beta_{0}$ are the same, the difference between the mass-loss rates of these winds comes from different density structures. To illustrate, consider cases S01 and S09 with $\beta_{0}=1$ : both cases present the same velocity profile and magnetic field configuration (although the intensity is not equal); but the density, and consequently the mass-loss rate, of S09 is 400 times larger than S01 in the entire numerical domain.

\subsection{The Third Set of Simulations with $\gamma=1.1$}

Throughout this paper, we have assumed that $\gamma=1.01$. However, more realistic magnetized wind models compute the heating of the wind caused due to physical processes, as is the case of the one-dimensional models done by Vidotto \& JatencoPereira (2006), Falceta-Gonçalves et al. (2006), Cranmer et al. (2007), among others. However, inclusion of such processes,
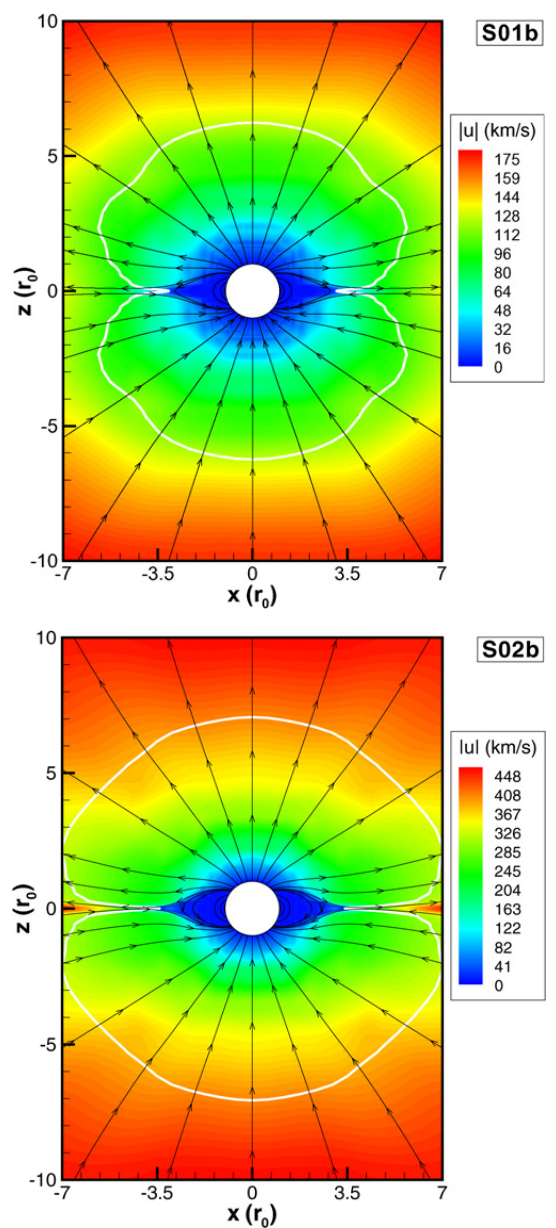

Figure 12. Same as Figure 4, but for simulations S01b and S02b, where $\gamma=1.1$. (A color version of this figure is available in the online journal.)

e.g., dissipation of waves or turbulence, in a three-dimensional code is very challenging. We thus opted to parameterize the energy content of the wind by the use of $\gamma$.

We investigate now how the wind structure will change if a different $\gamma$ is considered. Considering the cases S01/ S09 and S02/S08, where $\beta_{0}=1,1 / 25$, respectively, we performed simulations considering $\gamma=1.1$. This third set of simulations is presented in Table 1 and the meridional cuts of the steady-state configurations for $\mathrm{S} 01 \mathrm{~b}$ and $\mathrm{S} 02 \mathrm{~b}$ are shown in Figure 12.

It is worth noting that by adopting a different $\gamma$, the acceleration mechanism of the wind changes, what changes the velocity profile of the wind and, consequently, the configuration of the magnetic field (compare, for instance, the steady-state configuration of S01 and S01b). This is expected because, the higher $\gamma$ is, the thermal acceleration mechanism is less effective (e.g., there is less turbulence or dissipation of waves).

Due to the scaling relations described in Section 3.3, simulations $\mathrm{S} 01 \mathrm{~b}$ and $\mathrm{S} 09 \mathrm{~b}$, with $\beta_{0}=1$, present the same configuration of the steady-state wind. The same result is achieved for simulations $\mathrm{S} 02 \mathrm{~b}$ and $\mathrm{S} 08 \mathrm{~b}$, with $\beta_{0}=1 / 25$. 


\section{DISCUSSION AND CONCLUSION}

This is the first study to perform three-dimensional MHD numerical simulations analyzing in detail winds of magnetized solar-like stars with different $\beta_{0}$. As initial condition, our model assumes a bipolar configuration for the magnetic field at the stellar surface. This distribution of magnetic field, although very simplified, is useful as a first step in understanding how the interaction between stellar magnetic field and the wind occurs in a three-dimensional self-consistent manner.

In our simulations, we adopt a simpler treatment and parameterize the energy content of the wind in terms of $\gamma$. This is a first step toward more realistic simulations. It should be stressed that if one aims to describe the complex physics of stellar winds, a more complete description of the energy content of the wind has to be taken into account. In the SC, for instance, it is inferred that closer to the Sun, $\gamma \simeq 1$ (Steinolfson \& Hundhausen 1988), and at $1 \mathrm{AU}, \gamma \simeq 1.5$ (Totten et al. 1995; Phillips et al. 1995). Therefore, $\gamma$ is not expected to be constant throughout the stellar wind, as we assumed. As the aim of our study is to investigate the effects of the magnetic fields in the general properties of the stellar wind, the inclusion of MHD waves or variable $\gamma$ is postponed for future work.

In the first set of simulations where a fixed heating parameter $\gamma=1.01$ was adopted, we showed that for a solar-like star, the increase in the magnetic field intensity creates faster winds, with general characteristics as the PK71 result for the Sun: a creation of a bi-modal stellar wind. The final configuration of the magnetic field consists of a zone of closed magnetic loops at low latitudes. On top of the closed loop region lies the neutral point, where the reconnection takes place and is the starting point of the current sheet that extends for larger distances along the equatorial plane. The zone of open field lines located at high latitudes of the star fills all the space outside the closed loop region and carries along a wind.

For a given $\beta_{0}$, the wind is more accelerated in the polar regions than in the equatorial region. This difference is generated by the latitude-dependent Lorentz force which creates a flux of matter directed to the equatorial plane. This generates a density enhancement at low latitudes providing a mass-loss rate per unit solid angle that is also latitude-dependent and increases at low latitudes. This meridional flux is intensified for higher magnetic fields. The increase in the magnetic field also led to hotter winds with higher mass-loss rates.

We showed that the $\beta$-parameter is a key parameter in the structure of the wind in case $\gamma$ is the same for all simulations. As $\beta_{0}$ was increased back to 1 by changing $\rho_{0}$ and maintaining $B_{0}=20 \mathrm{G}$ in the second set of simulations, the wind decelerated and cooled. Comparing the first and second sets of simulations, we conclude that winds with same $\beta_{0}$ have (1) same velocity profile and (2) same magnetic field topology (the neutral point is located at the same position). However, mass-loss rates are different as $\dot{m}(\theta)$ is dependent on the choice of $\rho_{0}$.

By analyzing the effects of a different $\gamma$ in the simulations, we showed in the third set of simulations that the heating parameter $\gamma$ is, together with the plasma- $\beta$ parameter at the coronal base, an important parameter in the structure of the wind. However, the steady-state configuration of the wind in the third set is different from the first and second ones because the thermal force driving the wind changes when $\gamma$ changes.

In the simulations we ran, we adopted $\gamma=1.01$ in the first and second sets, and $\gamma=1.1$ in the third set. Although $\gamma$ is necessary for the thermal acceleration of the wind, a wind with a low $\gamma$ implies a proportionally high Lorentz force, when compared to the thermal force. For this reason, the third set of simulations is more asymmetrical than the first set (compare S01 and S01b, S02 and S02b), since the Lorentz force is $\theta$-dependent. Another aspect is that in a purely HD coronal wind, the thermal force is the main responsible for the acceleration of the wind. This implies that by adopting a low $\gamma$, the wind has less internal energy available, being less thermally accelerated. In the cases S01 $(\gamma=1.01)$ and S01b $(\gamma=1.1)$, the same situation holds: case $\mathrm{S} 01 \mathrm{~b}$ is less accelerated than case S01. However, cases S02 $(\gamma=1.01)$ and S02b $(\gamma=1.1)$ show a different behavior. The present simulations indicate that a change in gamma from 1.01 to 1.1 decreases the terminal speed by $43 \%$ in cases $\mathrm{S} 01 / \mathrm{S} 01 \mathrm{~b}$ and increases $8 \%$ in cases S02/S02b. This different behavior is because in case S02b, the Lorentz force provides a significant acceleration mechanism for the wind, i.e., the Lorentz force is proportionally more important than the thermal force. This illustrates the need to perform full three-dimensional MHD simulations in order to assess the importance of $\beta$ and $\gamma$.

The effective temperature of solar-like stars is orders of magnitude lower than the coronal temperature adopted in our models. In the present work, we did not take into account what is heating the wind from photospheric temperatures up to coronal temperatures. Instead, we placed the inner boundary of our model at the coronal base. For a more realistic study, however, the energy equation should be solved starting from lower layers. Hence, the plasma- $\beta$ parameter at the coronal base would then be incorporated as resultant from the energy interactions happening in these lower layers rather than being a free parameter at the coronal base, as in our models. Models and one-dimensional simulations that resolve the energy balance in the chromosphere-corona transition region have been calculated recently for the solar wind (Suzuki \& Inutsuka 2005; Cranmer et al. 2007) and stellar winds (Suzuki 2007; Cranmer 2008). If incorporated in three-dimensional global simulations, these models can provide physical insights on the processes that accelerate and heat stellar winds.

The present work considered moderate $\beta_{0}$. In the presence of a weak magnetic field (high $\beta_{0}$ ), the wind is energetic enough to drag the field lines with it, leading to a configuration of radial magnetic field lines. Consequently, a spherical expansion of the corona is expected, similar to a purely HD case.

Here, we deal with a class of non-rotating stars. The validity of our results should be tested also in rotating objects, since a more generalized description of magnetized outflows from solar-like stars requires the inclusion of rotation. The detailed interaction including rotation requires detailed three-dimensional MHD simulations. A future work will extend the present work to this direction.

A.A.V. thanks the Brazilian agencies FAPESP and CAPES for financial supports under grants 04-13846-6 and BEX4686/ 06-3, respectively. A.A.V. also thanks the warm reception at George Mason University where part of the work was performed. M.O. acknowledges the support by National Science Foundation CAREER grant ATM-0747654, and FAPESP support (2007/58793-5) during her visits to Brazil. M.O. is also thankful for the hospitality of University of São Paulo. V.J.-P. thanks CNPq under grant 305905/2007-4. We would like to thank the staff at NASA Ames Research Center for the use of the Columbia supercomputer and the anonymous referees for comments and suggestions that helped improve the manuscript. 


\section{REFERENCES}

Airapetian, V. S., Ofman, L., Robinson, R. D., Carpenter, K., \& Davila, J. 2000, ApJ, 528, 965

Bagnulo, S., Szeifert, T., Wade, G. A., Landstreet, J. D., \& Mathys, G. 2002, A\&A, 389, 191

Cohen, O., et al. 2007, ApJ, 654, L163

Cranmer, S. R. 2008, ApJ, 689, 316

Cranmer, S. R., \& van Ballegooijen, A. A. 2005, ApJS, 156, 265

Cranmer, S. R., van Ballegooijen, A. A., \& Edgar, R. J. 2007, ApJS, 171, 520

Falceta-Gonçalves, D., Vidotto, A. A., \& Jatenco-Pereira, V. 2006, MNRAS, 368,1145

Groth, C. P. T., De Zeeuw, D. L., Gombosi, T. I., \& Powell, K. G. 2000, J. Geophys. Res., 105, 25053

Hansen, K. C., Ridley, A. J., Hospodarsky, G. B., Achilleos, N., Dougherty, M. K., Gombosi, T. I., \& Tóth, G. 2005, Geophys. Res. Lett., 32, 20

Jatenco-Pereira, V., \& Opher, R. 1989, A\&A, 209, 327

Jones, G. H., Balogh, A., \& Forsyth, R. J. 1998, Geophys. Res. Lett., 25, 3109

Keppens, R., \& Goedbloed, J. P. 1999, A\&A, 343, 251

Keppens, R., \& Goedbloed, J. P. 2000, ApJ, 530, 1036 Zeeuw, D. L., Ridley, A., \& Gombosi, T. I. 2007, J. Geophys. Res. (Space Phys.), 112, 10210

Lima, J. J. G., Priest, E. R., \& Tsinganos, K. 2001, A\&A, 371, 240

Linde, T. J., Gombosi, T. I., Roe, P. L., Powell, K. G., \& Dezeeuw, D. L. 1998, J. Geophys. Res., 103, 1889

Liu, Y. C.-M., et al. 2008, ApJ, 680, 757

Low, B. C., \& Tsinganos, K. 1986, ApJ, 302, 163

Lugaz, N., Manchester, W. B., IV, \& Gombosi, T. I. 2005, ApJ, 627, 1019

Manchester, W. B., Gombosi, T. I., Roussev, I., De Zeeuw, D. L., Sokolov, I. V., Powell, K. G., Tóth, G., \& Opher, M. 2004, J Geophys. Res. (Space Phys.), 109,1102

Matthaeus, W. H., Elliott, H. A., \& McComas, D. J. 2006, J. Geophys. Res. (Space Phys.), 111, 10103

Matthaeus, W. H., Zank, G. P., Oughton, S., Mullan, D. J., \& Dmitruk, P. 1999, ApJ, 523, L93

McComas, D. J., Barraclough, B. L., Gosling, J. T., Hammond, C. M., Phillips, J. L., Neugebauer, M., Balogh, A., \& Forsyth, R. J. 1995, J. Geophys. Res., 100,19893
Mestel, L. 1968, MNRAS, 138, 359

Nerney, S. F., \& Suess, S. T. 1975, ApJ, 196, 837

Opher, M., Liewer, P. C., Gombosi, T. I., Manchester, W., DeZeeuw, D. L., Sokolov, I., \& Toth, G. 2003, ApJ, 591, L61

Opher, M., et al. 2004, ApJ, 611, 575

Parker, E. N. 1958, ApJ, 128, 664

Petit, P., et al. 2005, MNRAS, 361, 837

Phan-Bao, N., Martín, E. L., Donati, J.-F., \& Lim, J. 2006, ApJ, 646 L73

Phillips, J. L., et al. 1995, Geophys. Res. Lett., 22, 3301

Pneuman, G. W., \& Kopp, R. A. 1971, Sol. Phys., 18, 258 (PK71)

Powell, K. G., Roe, P. L., Linde, T. J., Gombosi, T. I., \& de Zeeuw, D. L. 1999, J. Comput. Phys., 154, 284

Reiners, A., \& Basri, G. 2006, ApJ, 644, 497

Ridley, A. J., de Zeeuw, D. L., Manchester, W. B., \& Hansen, K. C. 2006, Adv. Space Res., 38, 263

Robinson, R. D., Worden, S. P., \& Harvey, J. W. 1980, ApJ, 236, L155

Roussev, I. I., et al. 2003, ApJ, 595, L57

Sauty, C., \& Tsinganos, K. 1994, A\&A, 287, 893

Schrijver, C. J. 2001, ApJ, 547, 475

Steinolfson, R. S., \& Hundhausen, A. J. 1988, J. Geophys. Res., 93 14269

Suess, S. T., \& Smith, E. J. 1996, Geophys. Res. Lett., 23, 3267

Suzuki, T. K. 2007, ApJ, 659, 1592

Suzuki, T. K. \& Inutsuka, S.-i. 2005, ApJ, 632, L49

Tóth, G., Kovács, D., Hansen, K. C., \& Gombosi, T. I. 2004, J. Geophys. Res. (Space Phys.), 109, 11210

Totten, T. L., Freeman, J. W., \& Arya, S. 1995, J. Geophys. Res., 100, 13

Tsinganos, K., \& Trussoni, E. 1991, A\&A, 249, 156

Ud-Doula, A. 2003, PhD thesis, Univ. of Delaware

Velli, M. 1994, ApJ, 432, L55

Vidotto, A. A., \& Jatenco-Pereira, V. 2006, ApJ, 639, 416

Wade, G. A., Bagnulo, S., Drouin, D., Landstreet, J. D., \& Monin, D. 2007, MNRAS, 376, 1145

Washimi, H., \& Shibata, S. 1993, MNRAS, 262, 936

Weber, E. J., \& Davis, L. J. 1967, ApJ, 148, 217

Wilhelm, K. 2006, A\&A, 455, 697

Wood, B. E., Müller, H.-R., Zank, G. P., Linsky, J. L., \& Redfield, S. 2005, ApJ, $628, \mathrm{~L} 143$ 


\title{
SIMULATIONS OF WINDS OF WEAK-LINED T TAURI STARS: THE MAGNETIC FIELD GEOMETRY AND THE INFLUENCE OF THE WIND ON GIANT PLANET MIGRATION
}

\author{
A. A. Vidotto ${ }^{1,2}$, M. Opher $^{2}$, V. JATEnco-Pereira ${ }^{1}$, And T. I. Gombosi ${ }^{3}$ \\ ${ }^{1}$ University of São Paulo, Rua do Matão 1226, São Paulo, SP 05508-090, Brazil \\ ${ }^{2}$ George Mason University, 4400 University Drive, Fairfax, VA 22030-4444, USA; aline @ astro.iag.usp.br \\ ${ }^{3}$ University of Michigan, 1517 Space Research Building, Ann Arbor, MI 48109-2143, USA \\ Received 2009 May 6; accepted 2009 August 13; published 2009 September 11
}

\section{ABSTRACT}

By means of numerical simulations, we investigate magnetized stellar winds of pre-main-sequence stars. In particular, we analyze under which circumstances these stars will present elongated magnetic features (e.g., helmet streamers, slingshot prominences, etc). We focus on weak-lined T Tauri stars, as the presence of the tenuous accretion disk is not expected to have strong influence on the structure of the stellar wind. We show that the plasma- $\beta$ parameter (the ratio of thermal to magnetic energy densities) is a decisive factor in defining the magnetic configuration of the stellar wind. Using initial parameters within the observed range for these stars, we show that the coronal magnetic field configuration can vary between a dipole-like configuration and a configuration with strong collimated polar lines and closed streamers at the equator (multicomponent configuration for the magnetic field). We show that elongated magnetic features will only be present if the plasma- $\beta$ parameter at the coronal base is $\beta_{0} \ll 1$. Using our self-consistent three-dimensional magnetohydrodynamics model, we estimate for these stellar winds the timescale of planet migration due to drag forces exerted by the stellar wind on a hot-Jupiter. In contrast to the findings of Lovelace et al., who estimated such timescales using the Weber and Davis model, our model suggests that the stellar wind of these multicomponent coronae are not expected to have significant influence on hot-Jupiters migration. Further simulations are necessary to investigate this result under more intense surface magnetic field strengths $(\sim 2-3 \mathrm{kG})$ and higher coronal base densities, as well as in a tilted stellar magnetosphere.

Key words: magnetic fields - methods: numerical - MHD - planets and satellites: general - stars: pre-main sequence - stars: winds, outflows

Online-only material: color figures

\section{INTRODUCTION}

$\mathrm{T}$ Tauri stars are recently formed low-mass stars $(0.5 \lesssim$ $M / M_{\odot} \lesssim 2$ ), with spectral type ranging from $\mathrm{F}$ to $\mathrm{M}$, and radius of no more than 3-4 $R_{\odot}$. They are the predecessors of solarlike stars that are still in the pre-main-sequence (PMS) phase. Usually, T Tauri stars are classified in two categories: classical and weak-lined. A classical T Tauri star (CTTS) shows strong emission line activity, excess ultraviolet and infrared emissions, which are associated with accretion flows and the presence of circumstellar disks. A weak-lined T Tauri star (WTTS), on the other hand, has a weaker emission line activity and shows little or no trace of accretion, implying that the accretion disk has been dissipated. For this reason, they are also known as naked T Tauri stars.

WTTSs also show radio and X-ray flare activity that is usually explained as a scaled-up magnetic activity similar to the one observed in the Sun. In the Sun, highly detailed observations of the magnetic field, using both in situ and remote measurements, have shown a very complex magnetic configuration (McComas et al. 1995; Lin et al. 2005; Susino et al. 2008). Although in less detail, magnetic activity has also been observed in other stars (e.g., Petit et al. 2005; Phan-Bao et al. 2006; Reiners \& Basri 2006; Wade et al. 2007; Johns-Krull 2007; Valyavin et al. 2008; Donati et al. 2008b). As observational techniques are becoming more accurate, detection of a more complex magnetic field geometry in young stars has just started to be accomplished, for example, the CTTSs V2129 Oph (Donati et al. 2007), BP Tau
(Donati et al. 2008a), and CV Cha \& CR Cha (Hussain et al. 2009) where surface magnetic maps have been derived from spectropolarimetric data. Another system, V773 Tau A, a young binary system consisted of two WTTSs, is known to posses high levels of magnetic activity (Phillips et al. 1991, 1996). Recently, Massi et al. (2008) observed two extended radio emissions that were interpreted as associated to the primary and secondary stars of the system. They suggested that these features were produced by helmet streamer structures from each of the stars, that when overlapped at certain orbital phases, would result in reconnection producing the giant flares that were observed: radio emission would increase from a few $\mathrm{mJy}$ at apastron to more than $100 \mathrm{mJy}$ at periastron. In another recent work, Skelly et al. (2008) reported the findings of slingshot prominences on TWA6, another WTT star. In their interpretation, such magnetic prominence extends for about 4 stellar radii, which is beyond the corotation radius located at 2.4 stellar radii. Magnetic field extrapolations from surface magnetograms have also shown that the geometry of the large-scale field of T Tauri stars may be very complex (Jardine et al. 2008; Gregory et al. 2008).

In the present work, we aim to investigate the magnetic configuration of the corona of WTTSs using three-dimensional (3D) magnetohydrodynamics (MHD) numerical simulations. $\mathrm{T}$ Tauri stars are believed to be magnetized, and rotating several times faster than the Sun, hence it is important to consider a magneto-centrifugal wind.

The first theoretical models to analyze the effects of both rotation and magnetic field on stellar winds are Weber \& Davis 
(1967, WD from now on) and Mestel (1968), who investigated the coupling of a purely radial stellar magnetic field to the wind using MHD approach. Recently, theoretical and numerical efforts were made toward the study of more realistic magnetocentrifugal winds in different systems. For instance, Washimi \& Shibata (1993) studied the influence of stellar rotation on the wind structure and acceleration of magnetized main-sequence solar-like stars investigating the effects of the centrifugal force. Holzwarth (2005), on the other hand, analyzed the impact of a purely radial field on the wind. The stellar field, although radial, was taken to have a nonuniform distribution in the stellar surface, as to account for the nonuniform surface magnetic flux distribution observed in active stars. Matt \& Pudritz (2008) aiming to explain the low rotation rates observed in CTTSs, studied the role of an accretion powered stellar wind in the spinning down of a typical CTTS.

All these works have confirmed that the structure of a magnetized stellar wind significantly depends on both the field intensity and topology. In Vidotto et al. (2009), we performed an analysis of coronal winds in main-sequence solar-like stars. We showed that a decisive parameter in the acceleration of the magnetized wind of a solar-like star is the plasma- $\beta$ at the coronal base. Using 3D MHD self-consistent simulations, we explored how the change in the magnetic and thermal energy densities at the base of the wind modifies the magnetic configuration of the corona and the velocity profile of the wind. Rotation was not considered in that work.

We now investigate the magnetic configuration of the winds of WTTSs. In our models, we vary different physical parameters of the systems, such as stellar rotation period, and magnetic field intensity and gas density at the base of the wind. In particular, we investigate the occurrence of helmet streamers or slingshot prominences, as well as their shape and extension. We perform self-consistent time-dependent 3D numerical simulations, including centrifugal, magnetic, gravitational, and thermal forces. The full set of MHD equations is solved to obtain the steady state solution for the large-scale magnetic configuration of a T Tauri star.

The paper is organized as follows. In Section 2, we present the numerical scheme used; in Section 3, we describe our choice of parameters used in the simulations; and in Section 4, we present and discuss the results obtained. In Section 5, we analyze planetary migration due to drag forces exerted by the magnetized stellar wind on "hot-Jupiters," giant planets orbiting very close to the host star ( $\lesssim 0.1 \mathrm{AU})$. Section 6 is dedicated to conclusions and discussion.

\section{THE NUMERICAL MODEL}

To perform the simulations, we make use of the BlockAdaptive Tree Solar-wind Roe Upwind Scheme (BATS-RUS), a 3D MHD numerical code developed at the Center for Space Environment Modeling at University of Michigan (Powell et al. 1999). BATS-R-US has a block-based computational domain, consisting of Cartesian blocks of cells that can be adaptively refined for the region of interest. It has been used to simulate the heliosphere (Roussev et al. 2003), the outer heliosphere (Linde et al. 1998; Opher et al. 2003, 2006, 2007), coronal mass ejections (Manchester et al. 2004; Lugaz et al. 2005), the Earth's magnetosphere (Ridley et al. 2006), and the magnetosphere of other planets (Tóth et al. 2004; Hansen et al. 2005), among others. In this work, we extend the model developed in Vidotto et al. (2009) to study the wind structure of WTTSs.
BATS-R-US solves the ideal MHD equations, which in the conservative form are given by

$$
\begin{gathered}
\frac{\partial \rho}{\partial t}+\nabla \cdot(\rho \mathbf{u})=0, \\
\frac{\partial(\rho \mathbf{u})}{\partial t}+\nabla \cdot\left[\rho \mathbf{u} \mathbf{u}+\left(p+\frac{B^{2}}{8 \pi}\right) I-\frac{\mathbf{B} \mathbf{B}}{4 \pi}\right]=\rho \mathbf{g}, \\
\frac{\partial \mathbf{B}}{\partial t}+\nabla \cdot(\mathbf{u} \mathbf{B}-\mathbf{B} \mathbf{u})=0 \\
\frac{\partial \varepsilon}{\partial t}+\nabla \cdot\left[\mathbf{u}\left(\varepsilon+p+\frac{B^{2}}{8 \pi}\right)-\frac{(\mathbf{u} \cdot \mathbf{B}) \mathbf{B}}{4 \pi}\right]=\rho \mathbf{g} \cdot \mathbf{u},
\end{gathered}
$$

where $I$ is the identity matrix, $\rho$ the mass density, u the plasma velocity, $\mathbf{B}$ the magnetic field, $p$ the gas pressure, $\mathbf{g}$ the gravitational acceleration due to the central body, and $\varepsilon$ the total energy density given by

$$
\varepsilon=\frac{\rho u^{2}}{2}+\frac{p}{\gamma-1}+\frac{B^{2}}{8 \pi}
$$

We consider ideal gas, so $p=\rho k_{\mathrm{B}} T /\left(\mu m_{p}\right)$, where $k_{\mathrm{B}}$ is the Boltzmann constant, $T$ is the temperature, $\mu m_{p}$ is the mean mass of the particle, and $\gamma$ is the ratio of the specific heats. Due to the lack of knowledge of all the detailed processes that take place in a stellar wind, it is difficult to estimate all the mechanisms that modify the heat content of the wind (e.g., conduction, radiation, mechanical dissipation of energy that is transferred to the plasma). We adopt, therefore, an approach similar to Roussev et al. (2003) who considered that $\gamma$ is associated with "turbulent" internal degrees of freedom, in a way analogous to the Sun, where a significant amount of energy is stored in the form of waves and turbulent fluctuations.

For all the simulations performed, we adopted the same grid resolution. Initially, the simulation domain is refined in five levels. Other five refinement levels that are body-focused and focused on the equatorial plane (current sheet region) are applied next. Finally, an additional level is applied to the body. There are $9.1 \times 10^{6}$ cells in the domain. The smallest cell size is $0.018 R_{*}$, located around the central star, where $R_{*}$ is the stellar radius. The maximum cell size is $4.68 R_{*}$. The cell size near the current sheet is $0.036 R_{*}$. The grid is Cartesian and the star is placed at the origin. The axes $x, y$, and $z$ extend from $-75 R_{*}$ to $75 R_{*}$. More details of the grid can be found in Vidotto et al. (2009).

The inner boundary of the system is the base of the wind at $r=R_{*}$. Fixed boundary conditions were adopted at $r=R_{*}$. The outer boundary has outflow conditions, i.e., a zero gradient is set to all the primary variables.

The star is located at the center of the grid and has $M_{\star}=$ $0.8 M_{\odot}$ and $R_{*}=2 R_{\odot}$. The grid is initialized with a onedimensional hydrodynamic (HD), thermal pressure driven wind for a fully ionized hydrogen plasma. This solution is dependent on the choice of the temperature at the base of the wind and on $\gamma$, and the only physical possible solution is the one that becomes supersonic when passing through the critical radius (Parker 1958). The initial temperature profile obeys the polytropic relation $T \propto \rho^{\gamma-1}$. Due to conservation of mass of a steady wind, we obtain the density profile from the radial velocity profile $u_{r}(r)$.

The star is considered to be rotating as a solid body with a period of rotation $P$. The rotation axis is along the $z$-direction, parallel to the magnetic moment vector. The simulations are 
Table 1

The Set of Simulations

\begin{tabular}{lcccrc}
\hline \hline Name & $B_{0}(\mathrm{kG})$ & $P(\mathrm{~d})$ & $\gamma$ & $\rho_{0}\left(\mathrm{~g} \mathrm{~cm}^{-3}\right)$ & $\beta_{0}$ \\
\hline $\mathrm{A}$ & 1 & 3 & 1.2 & $1 \times 10^{-11}$ & $1 / 25$ \\
$\mathrm{~B}$ & 0.2 & 3 & 1.2 & $1 \times 10^{-11}$ & 1 \\
$\mathrm{C}$ & 1 & 3 & 1.1 & $1 \times 10^{-11}$ & $1 / 25$ \\
$\mathrm{D}$ & 1 & 10 & 1.2 & $1 \times 10^{-11}$ & $1 / 25$ \\
$\mathrm{E}$ & 1 & 3 & 1.2 & $2.4 \times 10^{-10}$ & 1 \\
$\mathrm{~F}$ & 1 & 3 & 1.2 & $2.4 \times 10^{-12}$ & $1 / 100$ \\
$\mathrm{G}$ & 1 & 0.5 & 1.2 & $2.4 \times 10^{-12}$ & $1 / 100$
\end{tabular}

Note. The columns represent, respectively: the name of the simulation, the surface magnetic field intensity at the pole, the period of rotation of the star, $\gamma$, the density at $R_{*}$, and the plasma- $\beta$ evaluated at the pole.

initialized with a dipolar magnetic field configuration described in spherical coordinates $\{r, \theta, \varphi\}$ by

$$
\mathbf{B}=\frac{B_{0} R_{*}^{3}}{r^{3}}\left(\cos \theta, \frac{1}{2} \sin \theta, 0\right),
$$

where $B_{0}$ is the magnetic field intensity at the poles, $r$ is the radial coordinate, $\theta$ is the colatitude, and $\varphi$ is the azimuthal angle measured in the equatorial plane. As the magnetic energy density is latitude dependent and the thermal pressure is held constant at the base of the wind, the plasma- $\beta$ at the surface of the star is minimum at the pole. The MHD solution is evolved in time from the initial dipolar configuration for the magnetic field to a fully self-consistent non-dipolar solution, until steady state is achieved.

\section{THE CHOICE OF PARAMETERS}

In this section, we describe the choice of the parameters used in the simulations. They can be seen in Table 1, where we present the maximum surface magnetic field intensity $B_{0}$, the stellar period of rotation $P$, the ratio of specific heats $\gamma$, the density at the base of the corona $\rho_{0}$, and $\beta_{0}$, the surface value of the plasma- $\beta$ evaluated at the pole. We chose to simulate extreme values of the stellar rotation period, magnetic field intensity, and density; in order to probe the possible magnetic configurations for WTTSs. A brief explanation of our choices is presented next.

Stellar rotation. It is believed that, after the dissipation of the disk, a low-mass PMS star spins up as it contracts toward the zero-age main sequence. Such belief of a disk-regulated rotational evolution during the PMS phase is supported by observations of the bimodal distribution of rotation periods (Bouvier et al. 1993; Choi \& Herbst 1996; Rebull et al. 2006). In samples of PMS stars from the Orion Nebula Cluster and NGC2264, Cieza \& Baliber (2007) found that stars with disks possess a distribution of periods peaking around $P \sim 8$ days, while stars without disks present the peak of the bimodal distribution at $P \sim 2$ days. To explore the extreme values of $P$, we chose periods ranging from 0.5 days to 10 days.

Coronal temperature. Over the last years, several observations, such as from the large X-ray surveys Chandra Orion Ultradeep Project (Getman et al. 2005) and XMM-Newton Extended Survey of the Taurus molecular cloud (Güdel et al. 2007), showed that the coronal temperature of TTSs can exceed $10^{7} \mathrm{~K}$. It is believed that the bulk of the X-ray emission is coronal, and it comes from the material confined inside closed magnetic field structures (Preibisch et al. 2005). However, the outflowing stellar wind is believed to be much cooler, as estimates presented by
Johns-Krull \& Herczeg (2007) from UV spectra of the CTTS TW Hya and by Gómez de Castro \& Verdugo (2007) for the CTTS RY Tau. In the present paper, our stellar wind models adopt a hot corona whose temperature at $R_{*}$ is $T_{0}=1 \times 10^{6} \mathrm{~K}$. Future works exploring latitudinal dependence of coronal heating, such as a prescribed heating for loops, will be explored.

Magnetic field intensity. High mean surface field strengths of up to a few $\mathrm{kG}$ have been observed in T Tauri stars (e.g., Johns-Krull 2007; Donati et al. 2007; Yang et al. 2008). Although the magnetic field configuration at the surface seems complex, a dipolar component should dominate at larger distances. A dipolar magnitude of few hundreds of $G$ has been detected (Valenti \& Johns-Krull 2004). In this work, we chose a magnetic field intensity in the range of 200-1000 G.

Ratio of specific heats. When detailed heating and cooling mechanisms are unknown, the energy budget of a stellar wind can be described by the use of a heating parameter $\gamma$ (Equation (5)). Near the Sun, for instance, due to a large amount of heating (e.g., by turbulence, dissipation of MHD waves, etc.), the solar plasma is considered as a gas with $\gamma \sim 1$ (Steinolfson $\&$ Hundhausen 1988). In T Tauri stars, the heating processes of the corona are poorly known. For simplicity, we parameterize the energy content of the wind of a WTTS by the use of $\gamma$, and we adopt $\gamma=1.1-1.2$.

Density. The range of densities we adopted for the base of the stellar wind is $2.4 \times 10^{-12}$ to $2.4 \times 10^{-10} \mathrm{~g} \mathrm{~cm}^{-3}$. This choice of density results in mass-loss rates ranging between $\sim 10^{-9}$ and $8 \times 10^{-8} M_{\odot} \mathrm{yr}^{-1}$.

\section{RESULTS: THE MAGNETIC FIELD CONFIGURATION}

In Figure 1, we present meridional plots for cases A, E, and $\mathrm{F}$ : the plots show contours of the poloidal velocity, the black lines are streamlines of magnetic fields, and the white lines represent the Alfvén surface (where the poloidal velocity of the wind equals the local Alfvén velocity). The first immediate conclusion is that the configuration of the magnetic field lines is very different in each case. Case E (Figure 1(a)) presents a dipolar-like configuration of the magnetic field lines. In case $\mathrm{F}$ (Figure 1(c)), the open field lines are more collimated in the polar region and in the equatorial region, we observe the formation of streamers. In the intermediate case A (Figure 1(b)), we find a larger region of closed field lines, coexisting with an open field line region emanating from high latitudes of the star. What differs between these three cases is the plasma- $\beta$, i.e., the ratio of the thermal to magnetic energy densities. For cases E, A, and $\mathrm{F}, \beta_{0}$ (evaluated at the pole) is respectively $1,1 / 25$, and $1 / 100$.

As discussed in Vidotto et al. (2009) in the context of nonrotating main-sequence solar-like stars, the plasma- $\beta$ is a decisive parameter in the acceleration of the wind. When the magnetic energy density is of the same order as the thermal energy density, as in case E (Figure 1(a)), the magnetic configuration remains approximately that of a dipole. Otherwise, when the magnetic energy density dominates over the thermal energy density, the wind is accelerated reaching a situation where the flow ram pressure is able to distort the initial magnetic configuration, as in case $\mathrm{F}$ (Figure 1(c)).

In addition to the acceleration of the flow, as a consequence of the Lorentz force, the wind velocity becomes more anisotropic with the decrease of $\beta_{0}$. Among the cases we analyzed, case $\mathrm{E}$ (and also B) presents velocity profiles resembling spherical symmetry and in case $\mathrm{F}$, where $\beta_{0}=1 / 100$ (high magnetic energy density), the wind is visibly divided into a region of fast speeds at high latitudes and slow speeds at low latitudes. 

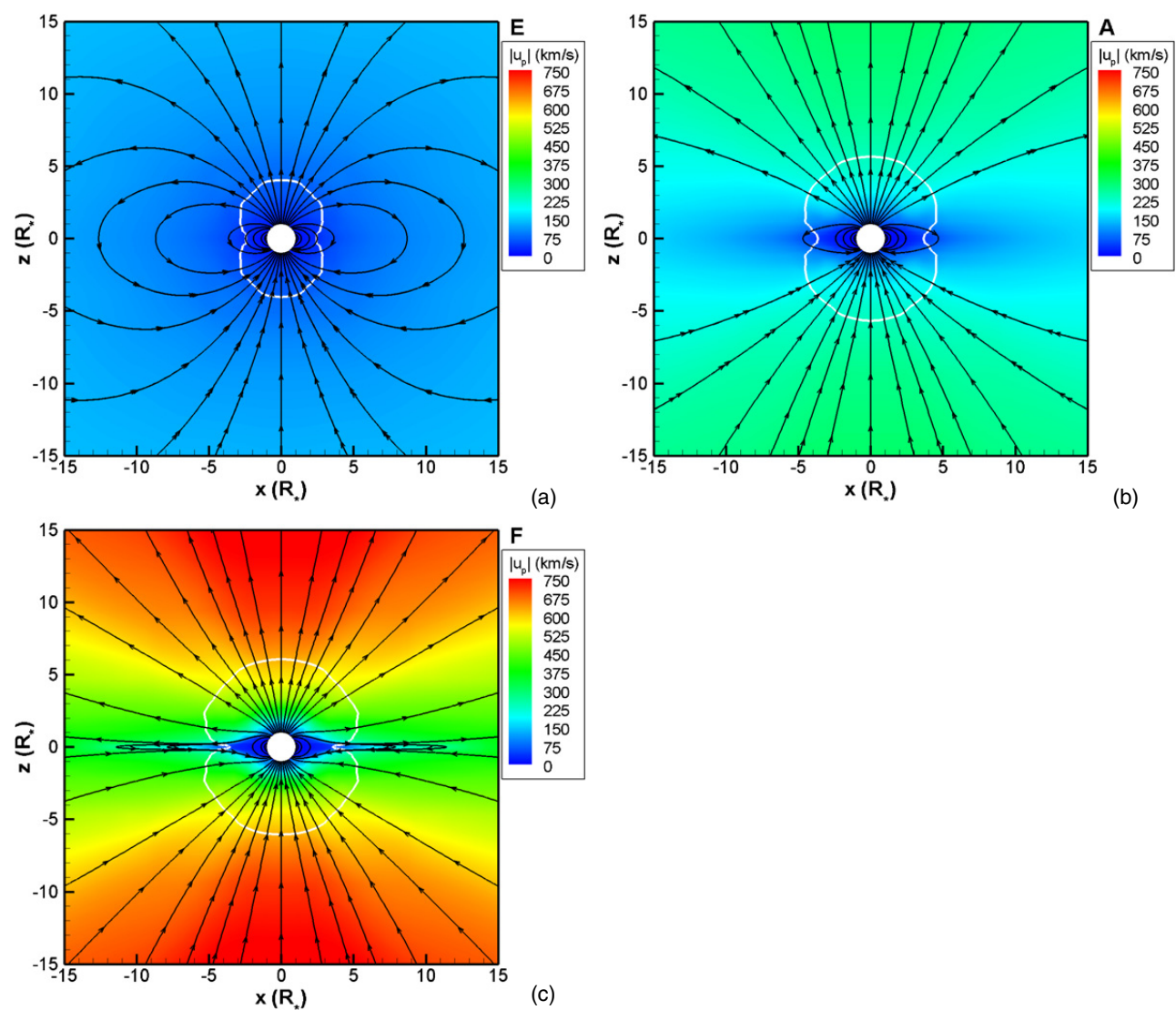

(b)

igure 1. Meridional cuts of poloidal velocity profiles for: (a) case

(A color version of this figure is available in the online journal.)

A comparison between cases A and F, where open field lines are found, shows that (1) case F shows higher collimation of lines toward the axis of rotation; and (2) the ratio of open to closed field line is larger in case F. As the magneto-centrifugal forces are responsible for the collimation of lines along the axis of rotation, it is expected that a wind more magnetically dominated would present higher levels of collimation (considering same stellar rotation rate). The reason why there are more open magnetic field lines in case F is mainly due to the flow speed: the high wind velocities stretch and open the previously closed field lines. As the polar wind is faster in case F, it is natural to expect a higher ratio of open to closed field line when compared to case $\mathrm{A}$.

These results also show that longer streamers will be present only if $\beta_{0} \ll 1$. The presence of elongated magnetic features has been recently considered to explain observations of recurrent radio flares in binary systems composed of T Tauri stars (Massi et al. 2008; Skelly et al. 2008). In these systems, the elongated magnetic features belonging to both stars could overlap in certain orbital phases, resulting in a modification of the magnetic configuration (reconnection). This picture could account for giant radio flares, emitted when the binary system is near periastron. Taking for example the system under consideration in Massi et al. (2008), the existence of elongated magnetic features indicates that $\beta_{0} \lesssim 0.01$. Adopting $B_{0} \sim 2600 \mathrm{G}$ and $T_{0} \sim 1 \mathrm{MK}$, it results in $n_{0} T_{0} \lesssim 2 \times 10^{19} \mathrm{~K} \mathrm{~cm}^{-3}$, or $\rho_{0} \lesssim 3.3 \times 10^{-11} \mu \mathrm{g} \mathrm{cm}^{-3}$, where $n_{0}$ is the number density at $R_{*}$ and $\mu$ is the mean molecular weight of the wind particles. Therefore, the observations of radio flares can help us constrain the coronal density in these stars.

PMS stars exhibit X-ray flares occurring in star sized, small structures, often interpreted as scaled-up solar-like coronal activities (Preibisch et al. 2005). In addition, intense X-ray flares are also observed. Favata et al. (2005) analyzed these intense X-ray flare decays to model physical parameters of the flaring structure, finding that these large flares take place in very long magnetic structures, extending out to several stellar radii. Getman et al. (2008) also argued that in fast rotating stars these powerful X-ray flares extend beyond the corotation radius, where centrifugal forces dominate over gravity. They conclude that the flares arise in traditional solar-type magnetic loops, where both footpoints are anchored in the stellar surface. Jardine \& van Ballegooijen (2005) developed a model for magnetic loops in rapidly rotating main-sequence stars that explains the existence of magnetic prominences that can extend out to heights of $2-5 R_{*}$, above the corotation radius, in equilibrium with the open field region. In our simulations, we observed an extended, slender closed field region in case F. In this case, the extension 

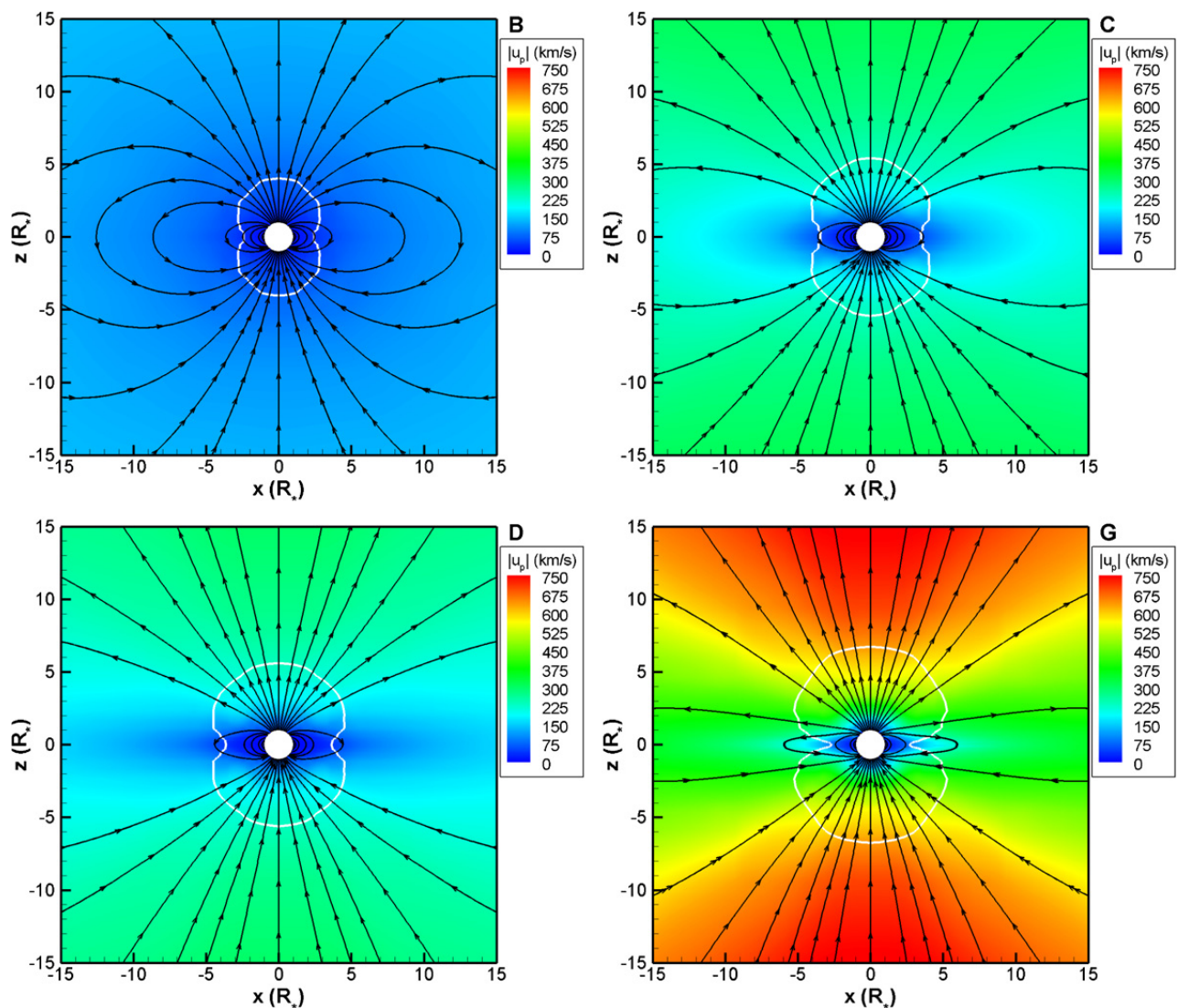

Figure 2. Same as Figure 1 for the remaining cases.

(A color version of this figure is available in the online journal.)

of the streamer is assured up to an equatorial radius of $\sim 13 R_{*}$. Above this point, the grid changes resolution, causing a local numerical reconnection (in principle, the loop could extend farther out). At the equatorial plane, the corotation radius is given by

$$
r_{\mathrm{co}}=\left(\frac{G M_{\star} P}{4 \pi^{2}}\right)^{1 / 3}=4.2 R_{\odot}\left(\frac{P}{1 \mathrm{~d}}\right)^{2 / 3}\left(\frac{M}{M_{\odot}}\right)^{1 / 3},
$$

which for the TTS simulated here is $r_{\mathrm{co}} / R_{*}=1.95 P_{d}^{2 / 3}$, where $P_{d}$ is the stellar rotation period measured in days. For $P=$ 3 days, $r_{\mathrm{co}} \simeq 4 R_{*}$. This shows that the elongated streamers, as obtained in case $\mathrm{F}$, is stable to at least $\sim 3$ times the corotation radius.

Figure 2 shows the steady state solutions for the remaining of the simulations presented in Table 1 . We enumerate the following conclusions. (1) We show that only a change in the rotation of the star does not provide a significant change in the poloidal velocity profile of the system (compare cases $\mathrm{A}$ and $\mathrm{D}, \mathrm{F}$ and $\mathrm{G})$. It changes, however, how the magnetic field lines are twisted: the faster is the rotation of the star, the larger is the $\varphi$-component of the magnetic field. (2) The heating parameter $\gamma$ influences the thermal acceleration of the wind (compare cases $\mathrm{A}$ and $\mathrm{C}$ ), being more accelerated in the case where the parameterized energy input is higher, i.e., for low $\gamma$.
As the wind characteristics are different, the resultant interaction with the magnetic field is different: case $\mathrm{A}$ (high $\gamma$ ) presents a larger ratio of open to close lines than case $\mathrm{C}$ (case $\mathrm{C}$ has a larger closed field line region). (3) Cases $\mathrm{B}$ and $\mathrm{E}$ present the same characteristics, as they possess the same $\beta_{0}$ (Vidotto et al. 2009). Furthermore, because $\beta_{0}=1$, there is an equipartition of thermal and magnetic energy densities at the base of the corona, which results in lower velocities and field lines that are merely modified in relation to the initial configuration, resembling a dipole. (4) Case $G$ represents a star with a high rotation rate. Because of this, among the cases we analyzed, it is the case where the open field lines are more collimated toward the axis of rotation. Comparing it to case $F$, where the rotation rate is six times smaller, it can also be seen that the higher the rotation rate is, the less elongated are the closed field lines.

The Alfvén surface plays an essential role in the determination of the magnetic field configuration. In magneto-centrifugal winds, both $B_{\varphi} / B_{r}$ and $B_{\varphi} / B_{\theta}$ are expected to be small inside the Alfvén surface. Beyond the Alfvén surface, the $r$ and $\theta$ components of the magnetic field decay faster than the $\varphi$ component, causing the field lines to be twisted. Therefore, helmet streamers anchored on rotating stars are not radial. In Figure 3, we plot a 3D view of the region close to the star. As can be seen, the picture of approximately radial streamers, as observed in the Sun or in slowly rotating stars, is not anymore 


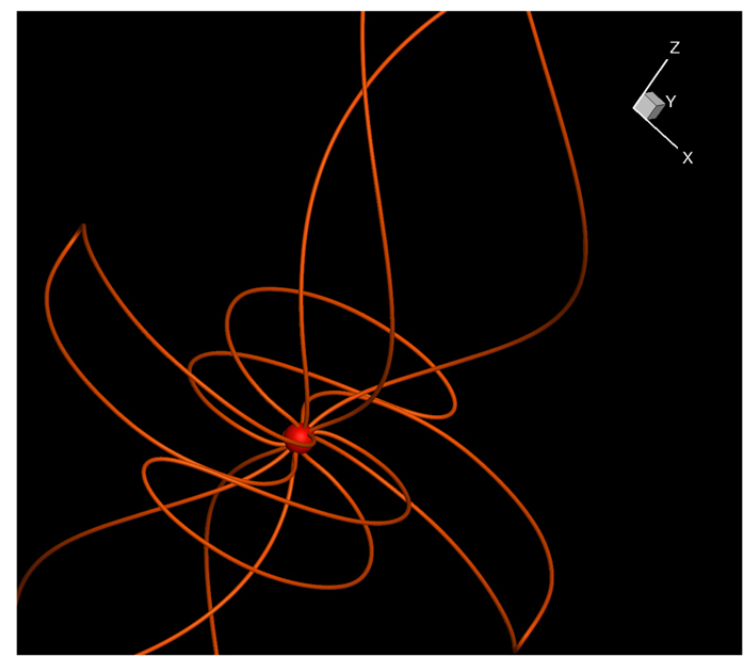

Figure 3. Three-dimensional view of the inner most region of case G. The lines plotted were selected as to outline the twisting of both closed and open magnetic lines anchored on the surface of the star.

(A color version of this figure is available in the online journal.)

verified in WTTSs with high rotation rates: both open and closed lines become twisted due to the stellar rotation.

Andre et al. (1988) presented an analytical expression for the calculation of the Alfvén surface, considering that (1) the wind should have spherical mass loss; (2) by the time the wind is crossing the Alfvén surface, it has already achieved the terminal velocity; and (3) the field is dipolar all the way to the Alfvén surface. As can be seen from our results, when the self-consistent interaction of the field lines and the wind is taken into account, these hypotheses may not be verified any longer.

Massi et al. (2008) computed the Alfvén radius of the wind of each of the stars in the binary system V 773 Tau A, where they observed helmet streamers, using Andre et al.'s (1988) analytical expression. They ruled out the twisting of magnetic lines of the helmet streamer, because in their estimate they found a large Alfvén surface with radius of $\sim 60 R_{*}$. According to our model, the Alfvén surface of each individual stellar wind is located much closer to the star and is probably even smaller than the minimum separation of the stars in the binary system $\mathrm{V} 773$ Tau A $\left(\sim 30 R_{*}\right)$, suggesting that the interacting streamers are probably twisted, as illustrated in Figure 3.

\section{STELLAR WIND EFFECTS ON PLANET MIGRATION}

In the previous section, we investigated the magnetic configuration of the wind of WTTSs. This configuration arises naturally from the interplay between the outflow and the field, i.e., the magnetic field is not restricted in our simulations. Several models assume a fixed magnetic field topology, as is the case of the Weber and Davis (WD) model. In this section, we investigate the effects our more realistic wind model will have on the migration of a giant planet.

According to the current favored planetary formation theory, the core of a giant planet forms far away from the central star, beyond the snow line ( $\gtrsim 5 \mathrm{AU}$, where the disk temperature falls below the condensation temperature of water) by coalescence of planetesimals (Pollack et al. 1996). When the solid core is sufficiently massive, it captures the surrounding gas of the accretion disk to form the atmosphere of a giant planet (Papaloizou \& Nelson 2005). However, observations show the existence of planets orbiting solar-like stars at very close distances $(\lesssim 0.1 \mathrm{AU})$. For this reason, these giant planets are often referred as hot-Jupiters. A possible explanation for this inconsistence is that the giant planet may have formed several AU from the central star, and later migrated inward. The interaction of the protoplanet with the disk wherein it was formed is a probable process that may drive this migration (Lin et al. 1996).

The inward migration is expected to cease when the planet is in the inner magnetospheric cavity, a cleared region between the stellar surface and the inner region of the accretion disk (Lin et al. 1996; Trilling et al. 1998; Papaloizou 2007). In recent numerical simulations, Romanova \& Lovelace (2006) found that for a stellar dipolar field that is not highly misaligned with the spin axis of the star and neither almost aligned so that 3D instabilities cannot fill the magnetospheric cavity with matter, the formation of the cleared region is assured. As in the cavity there is insufficient external material to remove the planet's angular momentum, the giant planet radial motions halt; the planet cannot grow further. In addition to this mechanism, others have been proposed to explain why the planet migration is inhibited all the way to the star (Lin et al. 1996; Fleck 2008).

In this section, in particular, we will investigate the action of magnetic torques from the stellar wind acting on the planet.

Considering that a T Tauri star has magnetic fields and rotation rates that are several times larger than in the Sun, Lovelace et al. (2008, LRB08 from now on) studied the torque that a magneto-centrifugally driven wind would cause on a close-in giant planet. They adopted the model of WD for the stellar wind and evaluated the change of the planet's angular momentum due to an azimuthal drag force exerted by the wind on the planet. Considering a solar mass star with $2 R_{\odot}, P \sim 3-5$ days, and $B_{0} \sim 1-3 \mathrm{kG}$, they found a timescale of $2-20 \mathrm{Myr}$ for a giant planet like Jupiter, orbiting the equatorial plane of the star, to have considerable radial motions.

Following LRB08, we estimate this timescale by adopting a more realistic stellar wind, product of our simulations. Consider a planet of mass $M_{p}$, orbiting very close to the star at a distance $r_{p}$. The angular momentum of the planet is then $L_{p}=M_{p} v_{K} r_{p}$, where $v_{K}=\left(G M_{\star} / r_{p}\right)^{1 / 2}$ is the azimuthal velocity of the planet, assumed Keplerian. Therefore, a change in the planet's angular momentum leads to

$$
\left|\frac{d L_{p}}{d t}\right| \simeq \frac{1}{2} M_{p} v_{K} \frac{d r_{p}}{d t} \simeq \frac{1}{2} M_{p} v_{K} \frac{r_{p}}{\tau_{w}},
$$

where $\tau_{w}$ is the timescale for changing significantly the planet's orbit due to the stellar wind drag (Lin et al. 1996). Another way to express the torque (Equation (8)) is to multiply the force the stellar wind will exert on the planet by the distance $r_{p}$ (LRB08)

$$
\left|\frac{d L_{p}}{d t}\right| \simeq\left(P_{\mathrm{ram}} A_{\mathrm{eff}}\right) r_{p},
$$

where $P_{\text {ram }}$ is the ram pressure the wind exerts on the planet

$$
P_{\text {ram }}=\frac{1}{2} \rho\left(u_{\varphi}-v_{K}\right)^{2}+\frac{1}{4 \pi} B^{2},
$$

and $A_{\text {eff }}$ is the cross section of the planet that intercepts the stellar wind. In order to take into account the effects that the magnetic field of the planet could have in the drag force 
of the stellar wind, LRB08 defined an effective cross section of the planet as

$$
A_{\mathrm{eff}}=\max \left[\pi R_{p}^{2}\left(\frac{B_{p}^{2} / 4 \pi}{P_{\mathrm{ram}}}\right)^{1 / 3}, \pi R_{p}^{2}\right],
$$

where $B_{p}$ is the surface magnetic field intensity at the pole of the planet and $R_{p}$ is its radius. The planet is assumed to have a dipolar-field configuration, and planetary mass loss is not considered. From Equations (8) and (9), we find

$$
\tau_{w} \simeq \frac{1}{2} \frac{M_{p} v_{K}}{P_{\mathrm{ram}} A_{\mathrm{eff}}} .
$$

A large $A_{\text {eff }}$ means that the cross section of the planet intercepting the wind is large, and thus, a more efficient drag mechanism (i.e., low $\tau_{w}$ ) is expected. If the wind ram pressure is large, the dragging mechanism is also expected to be important. However, a high $P_{\text {ram }}$ also implies in a small $A_{\text {eff }}$ (see Equation (11)).

The planet is supposed to be in the corotation radius, if the system is in a tidal equilibrium state (i.e., synchronized with the stellar rotation). However, Levrard et al. (2009) have shown that in the majority of the observed transiting extrasolar planets, the star's rotation is not synchronous. Due to this reason, we compute $\tau_{w}$ for a range of equatorial radial distances.

Considering the stellar winds from the simulations A, E, and $\mathrm{F}$, we calculated $\tau_{w}$ at the equatorial plane by assuming that the giant planet has the same mass and radius as Jupiter, and that the planet's magnetic field is $B_{p}=100 \mathrm{G}$ (same parameters adopted by LRB08). In order to perform the comparison of our results with the WD model, we evaluate Equation (12) for both models. We thus compute the velocity and magnetic field profiles of the stellar wind using the WD model. We assume the same parameters at the base of the wind as used in our simulations $\mathrm{A}, \mathrm{E}$, and $\mathrm{F}$, with the difference that $B_{0}$ is the magnetic field intensity at the pole in our model, and in the WD model it is the radial field calculated at the surface. We do the same procedure for the more realistic winds we simulate, i.e., we use the results of the simulations for $\mathbf{u}, \mathbf{B}$, and $\rho$ and evaluate $\tau_{w}$ using Equations (10)-(12). These results are shown in Figure 4, where the solid lines represent the results obtained using our model and the dashed lines for the WD model.

Figures 4(a) and (b) show, respectively, the effective cross section of the planet and the ram pressure exerted on the planet for the region inside $\sim 0.1 \mathrm{AU}$ (i.e., $\lesssim 10 R_{*}$ ). It can be seen that when $P_{\text {ram }}$ is large, $A_{\text {eff }}$ is small, and vice versa. In addition, $A_{\text {eff }}$ $\left(P_{\text {ram }}\right)$ calculated from our stellar wind model is larger (smaller) than if it were calculated using the WD model. This is because, for the WD model, $P_{\text {ram }}$ is verified to be essentially magnetic, i.e., the first term of the right-hand side (RHS) of Equation (10) is negligible compared to the second one, while for our stellar wind model, both terms are significant. In addition, for both models, the first term of the RHS of Equation (10) are of the same order. The difference thus comes from the magnetic term of Equation (10), which for the WD model is larger than for our model. This behavior is illustrated in Figure 5, for case F.

This results in larger timescales as compared to the WD model (Figure 4(c)), which implies that the more realistic winds simulated in the present paper are not expected to have significant influence on hot-Jupiter migrations. The timescales we estimate (e.g., $\gtrsim 50 \mathrm{Myr}$ for cases A and $\mathrm{F}$, for $r_{p} \gtrsim 3 R_{*}$ ) are much larger than the ones estimated by LRB08 $(\sim 2-$ $20 \mathrm{Myr})$. Other effects, such as an increase in the base density
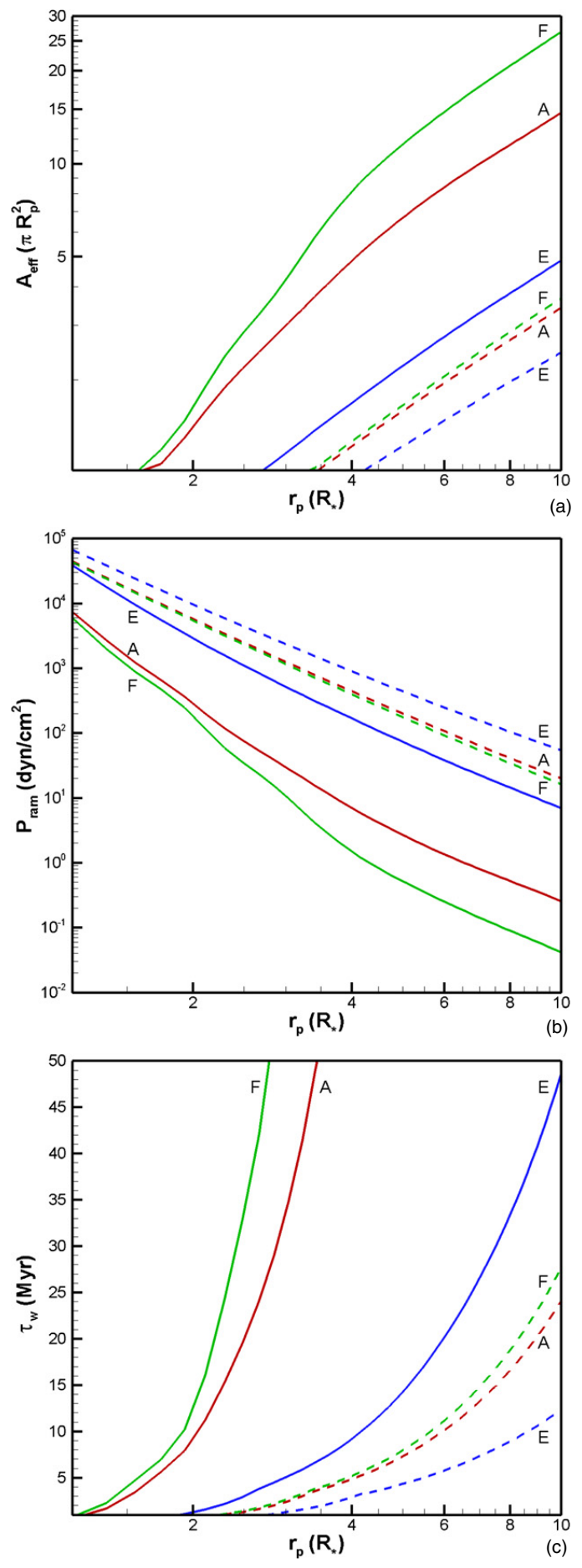

Figure 4. Comparison of: (a) the effective cross section of the planet that intercepts the stellar wind; (b) the wind ram pressure; and (c) the timescale for changing significantly the planet's orbit due to the stellar wind drag. We use the same parameters as in cases A (red lines), E (blue lines), and F (green lines): solid lines are the results for our stellar wind model, and dashed lines for the WD model.

(A color version of this figure is available in the online journal.) 


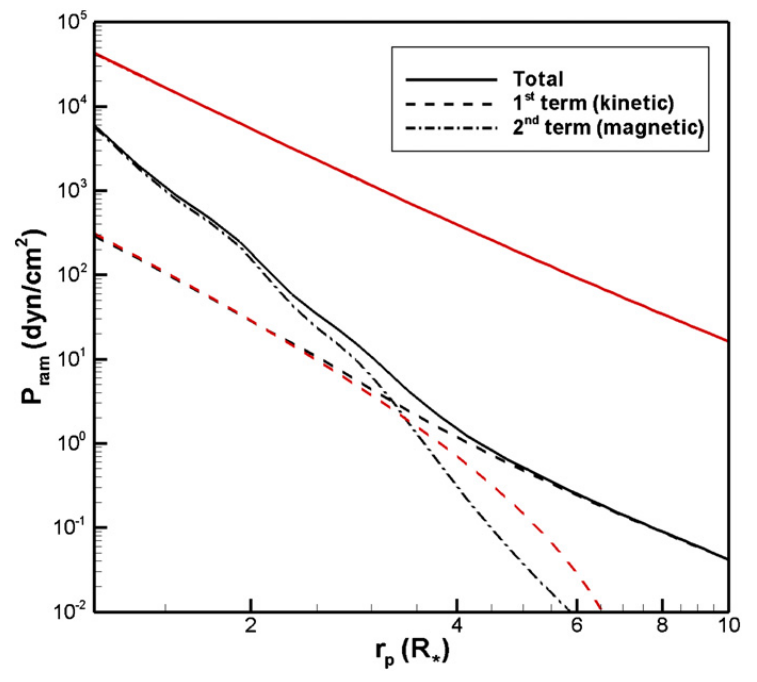

Figure 5. Relative importance of the first (dashed lines) and second (dot-dashed lines) terms in the RHS of Equation (10) calculated for case F using the results of our simulations (black) and using the WD model (red). Solid lines are the total ram pressures, i.e., the sum of first and second terms.

(A color version of this figure is available in the online journal.)

or magnetic field intensity, can reduce $\tau_{w}$. Case E presents the highest base density, while case F presents the lowest one among our simulations. From Figure 4(c), we note that an increase in $\rho_{0}$ reduces $\tau_{w}$. We expect that for $\rho_{0}>2.4 \times 10^{-10} \mathrm{~g} \mathrm{~cm}^{-3}$ with the remaining parameters as adopted in cases $\mathrm{A}, \mathrm{E}$, and $\mathrm{F}$, the effects of the migration will be more significant. A similar trend, i.e., a decrease in $\tau_{w}$, is also expected if one increases $B_{0}$. For example, cases $\mathrm{A}, \mathrm{E}$, and $\mathrm{F}$ assume $B_{0}=1 \mathrm{kG}$. If such value were increased, it would result in a larger ram pressure exerted by the wind on the planet. This would cause a more efficient drag and ultimately it would lower $\tau_{w}$, and perhaps it would even become smaller than $20 \mathrm{Myr}$ in the region where $r_{p} \lesssim 10 R_{*}$. In this case, the more realistic winds could have a more significant influence on the giant planet migration, though it would still have $\tau_{w}$ much larger than the one computed with the WD model.

According to LRB08, the magnetic winds are expected to be important for planet migration for the case of a multipolar magnetic field rather than a dipole field. In the present paper, we extend this statement to the case of the multi-field component coronae, as the ones analyzed previously, where open and closed field lines coexist. Our model suggests that the stellar wind of these modified dipole coronae are not expected to have significant influence on hot-Jupiters migration.

If the wind azimuthal velocity $u_{\varphi}$ is greater than the planet Keplerian velocity, LRB08 showed that the stellar wind causes the planet to move outward. In all the simulations we presented, this is the case only for case $\mathrm{G}$, for $r_{p} \gtrsim 1.26 R_{*}$. For all the other cases, the wind would act as to push the planet inward, and the closest the planet is from the star, the stronger/faster would be this dragging.

An aspect that is not investigated in the present paper is when the surface dipole is tilted with respect to the stellar rotation axis. The wind ram pressure is an interplay between magnetic $\left(\propto B^{2}\right)$ and kinetic terms $\left(\propto \rho\left[u_{\varphi}-v_{K}\right]^{2}\right)$. In the tilted case, a planet orbiting the rotation equator would be subjected to high magnetic field intensities (since $|\mathbf{B}|$ is maximum at the magnetic pole and minimum at the magnetic equator). However, it is unclear the latitudinal dependence of $u_{\varphi}$ : in the aligned case, $u_{\varphi}$ is maximum at the equator; in the misaligned case and in a highly magnetized ambient, the stellar magnetic field is expected to channel the rotating outflow. Hence, by a superposition of the magnetic and kinematic effects, it could be possible that $\tau_{w}$ becomes smaller in a tilted configuration. A future work will explore this scenario.

Another interesting configuration is when the orbital axis of the planet is inclined with respect to the spin axis of the star, as it is believed to occur, e.g., in the extrasolar planets HD80606b (Moutou et al. 2009) and XO-3b (Hébrard et al. 2008; Winn et al. 2009). In this configuration, the planet would interact with a stellar field strength that is larger at the position of the planet than at the magnetic equator. This could have an effect on $\tau_{w}$ that could reduce it.

\section{DISCUSSION AND CONCLUSION}

Recent works indicate the presence of complex magnetic structure, as the one observed in the Sun, on T Tauri stars. This structure is certainly influencing the winds of these stars. WTTS offers a tool for studying the magnetic behavior of low-mass PMS stars with the benefit that it is no longer strongly influenced by the presence of an accretion disk. As a consequence, the study of WTTS may eventually have applications on theories of CTTSs and also clues on the physical conditions for planetary formation and/or migration.

In the present work, we showed 3D MHD numerical simulations of magnetized stellar winds in rotating $\mathrm{T}$ Tauri stars. With the parameters we selected, we scan the possible values of rotation period ( $0.5-10$ days), magnetic field intensities ( $200 \mathrm{G}-1$ $\mathrm{kG})$, and surface density $\left(2.4 \times 10^{-12}\right.$ to $\left.2.4 \times 10^{-10} \mathrm{~g} \mathrm{~cm}^{-3}\right)$. We show that the plasma- $\beta$ parameter is a crucial parameter in the acceleration of the wind and in shaping the magnetic field lines. If the surface magnetic and thermal energy densities are equal at the pole, i.e., $\beta_{0}=1$, we showed that (1) the wind presents approximately spherical symmetry; (2) the magnetic field lines are not significantly distorted from the dipolar field that we assume as an initial condition of the simulation; and (3) the wind velocity does not differ considerably from the hydrodynamic case. On the other hand, if the magnetic energy density is much greater than the thermal one, i.e., $\beta_{0} \ll 1$, we observed departure of spherical symmetry. The wind shows two different components of the velocity, being faster at high latitudes and slower at low latitudes. In addition, the field lines are not anymore dipolar: the high velocity of the flow is able to stretch and open the field lines at high latitudes, while in low latitudes we observe elongated magnetic features. This indicates that streamer structures should be present in WTTSs with $\beta_{0} \ll 1$.

In a different context, ud-Doula \& Owocki (2002) defined a similar parameter that is used as an indicator of the effectiveness of magnetic fields in channeling outflow from hot supergiant stars. The "magnetic confinement parameter," defined by them as the ratio between the magnetic and kinetic wind energy densities, operates in a similar fashion as $\beta_{0}$ operates in our work: it identifies whether the magnetic field will play a significant role in the acceleration of the wind. However, both in the present work and in Vidotto et al. (2009), the thermal energy of the (coronal) wind is more significant then the kinetic energy, while in the line-driven winds studied by ud-Doula \& Owocki (2002), the kinetic energy is more important than the thermal energy of the wind. 
We also show that when the self-consistent interaction of the field lines and the wind is taken into account, the picture of approximately radial streamers (near the star), as observed in the Sun, is not anymore verified for the WTTSs with rotation periods up to 10 days: both open and closed lines are twisted.

With typical parameters of WTTSs, we estimate the timescale for planet migration due to the torque exerted by the azimuthal ram pressure of the stellar winds, and we showed that for the multicomponent corona of a WTTS simulated here, this migration mechanism is not important, in contrast to the results from LRB08, who argued that magnetic winds computed using the WD model are expected to be important for planet migration. Further simulations with a greater parameter range (higher magnetic field intensity or higher density at the coronal base) or different system configurations (a tilted stellar magnetosphere or a tilted planetary orbit with respect to the stellar spin axis) may reduce the timescale to significantly affect the orbital radius of the planet. For instance, if we consider a magnetic field intensity of 2-3 kG, still consistent with values derived from Zeemanbroadening measurements (Johns-Krull 2007), a reduction in $\tau_{w}$ is expected, as the ram pressure of the wind increases.

A.A.V. thanks the Brazilian agencies FAPESP (04-13846-6) and CAPES (BEX4686/06-3). A.A.V. also acknowledges the warm reception at George Mason University where part of the work was performed. M.O. acknowledges the support by National Science Foundation CAREER grant ATM-0747654. M.O. is also thankful for the hospitality of University of São Paulo. V.J.-P. thanks CNPq (305905/2007-4). The authors also thank the anonymous referee for valuable suggestions. The simulations presented here were performed at the Columbia supercomputer, at NASA Ames Research Center. This research has made use of NASA's Astrophysics Data System Bibliographic Services.

\section{REFERENCES}

Andre, P., Montmerle, T., Feigelson, E. D., Stine, P. C., \& Klein, K.-L. 1988, ApJ, 335,940

Bouvier, J., Cabrit, S., Fernandez, M., Martin, E. L., \& Matthews, J. M. 1993, A\&A, 272, 176

Choi, P. I., \& Herbst, W. 1996, AJ, 111, 283

Cieza, L., \& Baliber, N. 2007, ApJ, 671, 605

Donati, J.-F., et al. 2007, MNRAS, 380, 1297

Donati, J.-F., et al. 2008a, MNRAS, 386, 1234

Donati, J.-F., et al. 2008b, MNRAS, 390, 545

Favata, F., Flaccomio, E., Reale, F., Micela, G., Sciortino, S., Shang, H., Stassun, K. G., \& Feigelson, E. D. 2005, ApJS, 160, 469

Fleck, R. C. 2008, Ap\&SS, 313, 351

Getman, K. V., Feigelson, E. D., Micela, G., Jardine, M. M., Gregory, S. G., \& Garmire, G. P. 2008, ApJ, 688, 437

Getman, K. V., et al. 2005, ApJS, 160, 319

Gómez de Castro, A. I., \& Verdugo, E. 2007, ApJ, 654, L91

Gregory, S. G., Matt, S. P., Donati, J.-F., \& Jardine, M. 2008, MNRAS, 389, 1839

Güdel, M., et al. 2007, A\&A, 468, 353

Hansen, K. C., Ridley, A. J., Hospodarsky, G. B., Achilleos, N., Dougherty,

M. K., Gombosi, T. I., \& Tóth, G. 2005, Geophys. Res. Lett., 32, 20

Hébrard, G., et al. 2008, A\&A, 488, 763

Holzwarth, V. 2005, A\&A, 440, 411

Hussain, G. A. J., et al. 2009, MNRAS, 398, 198
Jardine, M. M., Gregory, S. G., \& Donati, J.-F. 2008, MNRAS, 386, 688

Jardine, M., \& van Ballegooijen, A. A. 2005, MNRAS, 361, 1173

Johns-Krull, C. M. 2007, ApJ, 664, 975

Johns-Krull, C. M., \& Herczeg, G. J. 2007, ApJ, 655, 345

Levrard, B., Winisdoerffer, C., \& Chabrier, G. 2009, ApJ, 692, L9

Lin, D. N. C., Bodenheimer, P., \& Richardson, D. C. 1996, Nature, 380, 606

Lin, J., Ko, Y.-K., Sui, L., Raymond, J. C., Stenborg, G. A., Jiang, Y., Zhao, S. \& Mancuso, S. 2005, ApJ, 622, 1251

Linde, T. J., Gombosi, T. I., Roe, P. L., Powell, K. G., \& Dezeeuw, D. L. 1998, J. Geophys. Res., 103, 1889

Lovelace, R. V. E., Romanova, M. M., \& Barnard, A. W. 2008, MNRAS, 389 , 1233 (LRB08)

Lugaz, N., Manchester, W. B., IV, \& Gombosi, T. I. 2005, ApJ, 627, 1019

Manchester, W. B., Gombosi, T. I., Roussev, I., De Zeeuw, D. L., Sokolov, I. V. Powell, K. G., Tóth, G., \& Opher, M. 2004, J. Geophys. Res. (Space Phys.), 109,1102

Massi, M., et al. 2008, A\&A, 480, 489

Matt, S., \& Pudritz, R. E. 2008, ApJ, 678, 1109

McComas, D. J., Barraclough, B. L., Gosling, J. T., Hammond, C. M., Phillips, J. L., Neugebauer, M., Balogh, A., \& Forsyth, R. J. 1995, J. Geophys. Res. 100,19893

Mestel, L. 1968, MNRAS, 138, 359

Moutou, C., et al. 2009, A\&A, 498, L5

Opher, M., Liewer, P. C., Gombosi, T. I., Manchester, W., DeZeeuw, D. L., Sokolov, I., \& Toth, G. 2003, ApJ, 591, L61

Opher, M., Stone, E. C., \& Liewer, P. C. 2006, ApJ, 640, L71

Opher, M., Stone, E. C., \& Gombosi, T. I. 2007, Science, 316, 875

Papaloizou, J. C. B. 2007, A\&A, 463, 775

Papaloizou, J. C. B., \& Nelson, R. P. 2005, A\&A, 433, 247

Parker, E. N. 1958, ApJ, 128, 664

Petit, P., et al. 2005, MNRAS, 361, 837

Phan-Bao, N., Martín, E. L., Donati, J.-F., \& Lim, J. 2006, ApJ, 646, L73

Phillips, R. B., Lonsdale, C. J., \& Feigelson, E. D. 1991, ApJ, 382, 261

Phillips, R. B., Lonsdale, C. J., Feigelson, E. D., \& Deeney, B. D. 1996, AJ, 111,918

Pollack, J. B., Hubickyj, O., Bodenheimer, P., Lissauer, J. J., Podolak, M., \& Greenzweig, Y. 1996, Icarus, 124, 62

Powell, K. G., Roe, P. L., Linde, T. J., Gombosi, T. I. \& de Zeeuw, D. L. 1999, J. Computat. Phys., 154, 284

Preibisch, T., et al. 2005, ApJS, 160, 401

Rebull, L. M., Stauffer, J. R., Megeath, S. T., Hora, J. L., \& Hartmann, L. 2006, ApJ, 646, 297

Reiners, A., \& Basri, G. 2006, ApJ, 644, 497

Ridley, A. J., de Zeeuw, D. L., Manchester, W. B., \& Hansen, K. C. 2006, Adv Space Res., 38, 263

Romanova, M. M., \& Lovelace, R. V. E. 2006, ApJ, 645, L73

Roussev, I. I., et al. 2003, ApJ, 595, L57

Skelly, M. B., Unruh, Y. C., Cameron, A. C., Barnes, J. R., Donati, J.-F., Lawson, W. A., \& Carter, B. D. 2008, MNRAS, 385, 708

Steinolfson, R. S., \& Hundhausen, A. J. 1988, J. Geophys. Res., 93, 14269

Susino, R., Ventura, R., Spadaro, D., Vourlidas, A., \& Landi, E. 2008, A\&A, 488, 303

Tóth, G., Kovács, D., Hansen, K. C., \& Gombosi, T. I. 2004, J. Geophys. Res (Space Phys.), 109, 11210

Trilling, D. E., Benz, W., Guillot, T., Lunine, J. I., Hubbard, W. B., \& Burrows, A. 1998, ApJ, 500, 428

ud-Doula, A. \& Owocki, S. P. 2002, ApJ, 576, 413

Valenti, J. A., \& Johns-Krull, C. M. 2004, Ap\&SS, 292, 619

Valyavin, G., Wade, G. A., Bagnulo, S., Szeifert, T., Landstreet, J. D., Han, I., \& Burenkov, A. 2008, ApJ, 683, 466

Vidotto, A. A., Opher, M., Jatenco-Pereira, V., \& Gombosi, T. I. 2009, ApJ, 699, 441

Wade, G. A., Bagnulo, S., Drouin, D., Landstreet, J. D., \& Monin, D 2007, MNRAS, 376, 1145

Washimi, H., \& Shibata, S. 1993, MNRAS, 262, 936

Weber, E. J., \& Davis, L. J. 1967, ApJ, 148, 217 (WD)

Winn, J. N., et al. 2009, ApJ, 700, 302

Yang, H., Johns-Krull, C. M. \& Valenti, J. A. 2008, AJ, 136, 2286 


\title{
A SELF-CONSISTENT DETERMINATION OF THE TEMPERATURE PROFILE AND THE MAGNETIC FIELD GEOMETRY IN WINDS OF LATE-TYPE STARS
}

\author{
A.A. VIDOTTO*, D. FALCETA-GONÇALVES and V. JATENCO-PEREIRA \\ Depto. de Astronomia, Univ. de São Paulo, Brazil \\ (*Author for correspondence, E-mail: aline@astro.iag.usp.br)
}

(Received 27 October 2005; Accepted 6 February 2006)

\begin{abstract}
Cool giant and supergiant stars generally present low velocity winds with high massloss rates. Several models have been proposed to explain the acceleration process of these winds. Although dust is known to be present in these objects, the radiation pressure on these particles is uneffective in reproducing the observed physical parameters of the wind. The most promising acceleration mechanism cited in the literature is the transference of momentum and energy from Alfvén waves to the gas. Usually, these models consider the wind to be isothermal. We present a stellar wind model in which the Alfvén waves are used as the main acceleration mechanism, and determine the temperature profile by solving the energy equation taking into account both the radiative losses and the wave heating. We also determine, self-consistently, the magnetic field geometry as the result of the competition between the magnetic field and the thermal pressure gradient. As the main result, we show that the magnetic geometry presents a super-radial index in the region where the gas pressure is increasing. However, this super-radial index is greater than that observed for the solar corona.
\end{abstract}

Keywords: stars: mass loss, stars: magnetic fields, MHD, waves

\section{Introduction}

After decades of theoretical and observational studies of cool giant and supergiant stars, the mechanisms by which the wind acceleration occurs are still poorly understood. Compared to the Sun, these stars are known to present continuous mass-loss process occurring at high rates, typically $10^{-10}$ to $10^{-5} \mathrm{M}_{\odot} \mathrm{yr}^{-1}$, but in low velocity winds $\left(u_{\infty}<300 \mathrm{~km} \mathrm{~s}^{-1}\right)$ (Dupree, 1986; Lamers and Cassinelli, 1999). Radiative pressure on grains transfers momentum to these particles being responsible for their acceleration and, if gas and dust are dynamically well coupled, grains drag the gas outwards resulting in the mass ejection. However, for stationary envelopes (e.g., pre-AGB phase) dust-driven theoretical models have lately failed in reproducing the wind properties, mainly because the dust-gas coupling is not effective (Sandin and Hofner, 2003). Observationally, Guandalini et al. (2005) found no strong correlation between the mass-loss rates and the luminosities of AGB stars. Their main conclusion is that, if radiative pressure is important in powering these stellar winds, it must occur in addition to other mechanism. Another drawback to the radiation pressure models is the need for the dust formation region to be close to the star.

Space Science Reviews (2006) 122: 181-188 
Recent high-resolution Doppler measurements show that winds are mainly accelerated near the stellar surface $\left(r<1.3 R_{*}\right)$ (Airapetian et al., 2003), while grains are expected to grow and survive at even larger distances.

In this sense, another mechanism must be used to accelerate the gas near the surface. The most promising mechanism for the winds of cool stars is the transference of momentum and energy to the wind from MHD waves. Hartmann and MacGregor (1980) showed that it would be possible to reproduce the observed low wind velocities and the high mass-loss rates of the cool giant and supergiant stars if some kind of wave-damping mechanism is effective at the wind basis $\left(r<2 R_{*}\right)$. Jatenco-Pereira and Opher (1989) studied the effects of different damping mechanisms and magnetic field divergence and they showed that the magnetic field divergent geometry can rapidly dilute the wave flux and also slow down the wind. Their magnetic field geometry was based on empirical relations found from observations of the solar wind. This is because in general, in lack of direct measurements of the magnetic field fluctuations and structure in other stars, we have to simply extrapolate our knowledge from solar observations.

In this work, we model the acceleration and heating of a late-type supergiant stellar wind considering an outwarded flux of Alfvén waves. We solve the MHD equations to, self-consistently, determine the magnetic field geometry and the wind temperature and velocity profiles. In Section 2, we describe the model basic equations. In Section 3, we present the results and the discussions, followed, by the work conclusions.

\section{The Model}

The basic wind equations are based on mass, momentum, energy, and magnetic flux conservation. The first is given by $\rho u A(r)=\rho_{0} u_{0} A\left(r_{0}\right)$, where $u$ is the flow velocity, $\rho$ is the gas density and $A(r)$ is the flow cross-sectional area at a distance $r$ from the center of the star. The index " 0 " indicates the variable is being evaluated at the stellar surface.

Assuming a steady flow, the momentum equation can be written as:

$$
\rho(\vec{u} \cdot \vec{\nabla}) \vec{u}=-\frac{\rho G M_{*}}{r^{3}} \vec{r}-\vec{\nabla}\left(P+\frac{\left\langle(\delta B)^{2}\right\rangle}{8 \pi}\right)+\frac{1}{4 \pi}(\vec{\nabla} \times \vec{B}) \times \vec{B}
$$

where $P=\rho k_{\mathrm{B}} T / m$ is the thermal pressure, $k_{\mathrm{B}}$ is the Boltzmann constant, $T$ is the gas temperature, $m$ is the mean mass per particle, $G$ the gravitational constant, and $\delta B$ the wave magnetic field amplitude. In Equation (1), the right hand side contains the gravitational force density and the thermal and the wave pressure gradients, respectively. The last term represent the magnetic force. The wave amplitude $(\delta B)$ is related to the wave energy density $(\epsilon)$ by $\epsilon=\left\langle(\delta B)^{2}\right\rangle / 4 \pi$. 


\subsection{Thin Flux Tube Approximation}

Typically, considering magnetic field strengths $>1 \mathrm{G}$, the wind basis is characterized by the relation $\beta=P /\left(B^{2} / 8 \pi\right) \ll 1$, i.e., the plasma is magnetically dominated. In this case, if we assume the wind to be initiated at funnels anchored at the stellar surface, which are surrounded by a plasma with lower magnetic field strength, the magnetic pressure inside will push the gas and the funnel field lines will expand. The funnel cross-section radius $(\mathcal{R})$ will grow super-radially up to a limit value $\left(\mathcal{R}_{m}\right)$. This limiting cross-section radius depends both on the relation between external and internal magnetic field strengths and on the filling factor $(\alpha)$. As the area increases, the internal magnetic strength diminishes until the equilibrium between internal and external magnetic pressures is reached. If the internal magnetic field strength is much larger than the external, the flux tubes cross-section will depend on the filling factor only. The filling factor is the ratio between the area of the stellar surface covered by funnels and the total area. The averaged maximum area that a funnel could reach would be $A_{m}=A\left(r_{0}\right) / \alpha$ or, in terms of the cross-section radius: $\mathcal{R}_{m}=\mathcal{R}_{0} / \alpha^{1 / 2}$. For the quiet Sun, the funnels that merge to form the coronal holes cover about $10 \%$ of the total surface.

In this work, at the wind basis, we assume the plasma to be magnetically dominated and the left-hand side of Equation (1) may be neglected if compared to the other terms. Then, by using $\vec{\nabla} \cdot \vec{B}=0$ and a power series expansion method proposed by Pneuman et al. (1986), we can determine, self-consistently, the magnetic field geometry without assuming any empirical function for the funnel cross-section with distance.

Following Pneuman et al. (1986), using the thin flux tube approximation, Equation (1) and $\vec{\nabla} \cdot \vec{B}=0$ are described near stellar surface, which in our model occurs up to $\sim 1.4 r_{0}$, by:

$$
\begin{aligned}
4 \pi \frac{\partial P}{\partial y} & \simeq B_{r}\left(\frac{\partial B_{y}}{\partial r}-\frac{\partial B_{r}}{\partial y}\right), \\
4 \pi\left(\frac{\partial P}{\partial r}+\frac{P}{H}\right) & \simeq-B_{y}\left(\frac{\partial B_{y}}{\partial r}-\frac{\partial B_{r}}{\partial y}\right),
\end{aligned}
$$

and

$$
\frac{1}{y} \frac{\partial}{\partial y}\left(y B_{y}\right)+\frac{\partial B_{r}}{\partial r}=0
$$

where $H=k_{\mathrm{B}} T r^{2} / G m M_{*}$ is the scale height. The thin flux tube approximation is reliable when the cross-section radius $\mathcal{R}$ is negligible compared to both the scale height of the external medium and any variation scales along the tube (Spruit, 1981; Longcope and Klapper, 1997). 
Expanding all variables as power series in $y$ (i.e., along the tube radius), and neglecting terms of orders higher than 2, Equations (2)-(4) give rise to a differential equation for the flux tube cross-section:

$$
\begin{gathered}
\frac{A\left(r_{0}\right)}{2 H_{0}^{2}}\left[\frac{\partial^{2}}{\partial r^{2}}\left(\frac{A\left(r_{0}\right)}{A(r)}\right)-\frac{1}{2 A(r)} \frac{\partial}{\partial r}\left(\frac{A\left(r_{0}\right)}{A(r)}\right)\right]= \\
\left(\frac{A\left(r_{0}\right)}{A(r)}\right)^{2}\left[1-\left(\frac{B_{\mathrm{ext}}}{B_{0}} \frac{(1-\alpha)}{\left(\frac{A\left(r_{0}\right)}{A(r)}-\alpha\right)}\right)^{2}\right]+2 \beta \frac{P(r)}{P\left(r_{0}\right)},
\end{gathered}
$$

where $\beta=4 \pi P\left(r_{0}\right) / B_{0}^{2}, \alpha$ is the filling factor and $B_{\text {ext }}$ is the magnetic field strength outside the flux tube. In the following calculations we fixed its value to be $10^{-3} B(r)$.

To simplify the set of equations, we will define the funnel area expansion as a function of radial distance by: $A(r)=A\left(r_{0}\right)\left(r / r_{0}\right)^{S}$, where $S$ is the expansion index, which is super-radial $(S>2)$ at the wind basis up to the merging radius when $S$ becomes 2. $S$ is determined from Equation (5).

\subsection{The Wind Equations}

In a consistent model, to avoid assuming any empirical function for the magnetic field geometry and to determine the wind temperature at each wind position $(r)$, we have to solve the energy equation, which is determined from the balance between wave heating and the adiabatic expansion and radiative coolings (Hartmann et al., 1982). Thus, neglecting conduction, we write the energy equation as:

$$
\rho u \frac{d}{d r}\left(\frac{u^{2}}{2}+\frac{5}{2} \frac{k_{\mathrm{B}} T}{m}-\frac{G M_{*}}{r}\right)+\frac{u}{2} \frac{d \epsilon}{d r}=\left(Q-P_{\mathrm{R}}\right),
$$

where $Q$ is the wave heating rate, i.e., the rate at which the gas is being heated due to dissipation of wave energy, and $P_{\mathrm{R}}$ is the radiative cooling rate, both in erg cm $\mathrm{cm}^{-3}$ $\mathrm{s}^{-1}$. The wave heating can be written as $Q=\epsilon\left(u+v_{\mathrm{A}}\right) / L$ and the radiative cooling is given by $P_{\mathrm{R}}=\Lambda n_{\mathrm{e}} n_{\mathrm{H}}$, where $n_{\mathrm{e}}$ is the electron density, $n_{\mathrm{H}}$ is the hydrogen density, and $\Lambda$ is the radiative loss function. Here, we adopt the $\Lambda$ function given by Schmutzler and Tscharnuter (1993) and calculate $n_{\mathrm{e}}$ with the modified Saha equation given by Hartmann and MacGregor (1980).

The wave energy density at each step may be calculated using a WKB approximation, from the wave action conservation. This approximation can often be employed when the properties of the medium vary slowly on a scale comparable to the wavelength (Usmanov et al., 2000). Under this assumption, the wave energy density is dissipated as follows:

$$
\epsilon=\epsilon_{0} \frac{M_{0}}{M}\left(\frac{1+M_{0}}{1+M}\right)^{2} \exp \left[-\int_{r_{0}}^{r} \frac{1}{L} d r^{\prime}\right]
$$


where $M=u / v_{\mathrm{A}}$ is the Alfvén-Mach number, $v_{\mathrm{A}}=(B / \sqrt{4 \pi \rho})$ the Alfvén speed and $L$ the wave-damping length. Also, the wave flux $\left(\phi_{\mathrm{A}}\right)$ at $r_{0}$ is evaluated by $\phi_{\mathrm{A}_{0}}=\epsilon_{0} v_{\mathrm{A}_{0}}\left(1+1.5 M_{0}\right)$. We consider the nonlinear damping mechanism, in which length is given by (Jatenco-Pereira and Opher, 1989):

$$
L=L_{0}\left(\frac{v_{\mathrm{A}}}{v_{\mathrm{A}_{0}}}\right)^{4} \frac{\left\langle(\delta v)^{2}\right\rangle_{0}}{\left\langle(\delta v)^{2}\right\rangle}(1+M)
$$

where $\left\langle(\delta v)^{2}\right\rangle$ is the averaged squared perturbation velocity amplitude and $L_{0}$ is the damping length at the wind basis, which is mainly dependent on the assumed wave frequency spectrum (Lagage and Cesarsky, 1983). Here, we will let it as a free parameter.

The equations presented here fully describe the wind parameters and the magnetic field geometry under the given assumptions. In the next section, we show the main results by applying these equations in a typical cool supergiant star and comparing them with previous works.

\section{Results and Discussions}

For the earlier decades, the validity of a wind model was limited to simply reproducing the terminal velocity and the mass-loss rate of a given star. As a consequence, a number of accelerating mechanisms were found in accordance with observations. However, with the appearance of high-resolution observations from more sensitive instruments, in the near future, the radial profiles of the wind parameters will become measurable and will be decisive on the choice of the wind model.

Here, we obtained the wind parameter profiles applying the described model on a cool supergiant star with $M_{*}=16 \mathrm{M}_{\odot}, r_{0}=400 R_{\odot}, \rho_{0}=10^{-13} \mathrm{~g} \mathrm{~cm}^{-3}, B_{0}=$ $10 \mathrm{G}$ and $T_{0}=3500 \mathrm{~K}$, as used by Hartmann and MacGregor (1980) in their model star 4 . We also assumed a filling factor $\alpha=0.1$, according to solar observations. For these stars, observational data reveal typical mass-loss rates of $\dot{M} \simeq 10^{-7}$ to $10^{-6} \mathrm{M}_{\odot} \mathrm{yr}^{-1}$ and terminal velocities of $u \simeq 70 \mathrm{~km} \mathrm{~s}^{-1}$. Unfortunately, the available data are limited in spatial resolution for most of the stars, and it is not possible to fit the complete radial profiles.

Assuming a surface magnetic field strength $B_{0}=10 \mathrm{G}$, an Alfvén waves flux of $\phi_{\mathrm{A}_{0}}=10^{7} \mathrm{erg} \mathrm{cm}^{-2} \mathrm{~s}^{-1}$ at the wind basis and a low damped wave flux $\left(L_{0}=5 r_{0}\right)$, both the wind terminal velocity and the mass-loss rate obtained were consistent with the observations. This value corresponds to a wave amplitude of $\sqrt{\left\langle(\delta B)^{2}\right\rangle} \simeq$ $3 \times 10^{-2} B_{0}$, which is very plausible for a turbulent medium as that at the stellar surface (Suzuki and Inutsuka, 2005).

The magnetic field geometries for both simplified and self-consistent models are shown in Figure 1. The velocity and temperature profiles for this case are shown in Figure 2. The velocity profile reveals a peak of $u>100 \mathrm{~km} \mathrm{~s}^{-1}$ at $r<2.0 r_{0}$, 


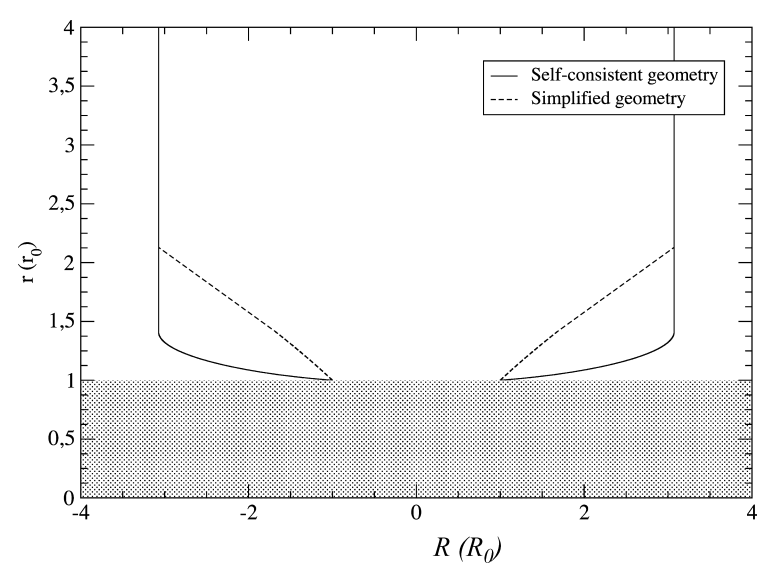

Figure 1. The magnetic field structure for a constant super-radial index $S=5$ (dashed line) and that determined self-consistently in our model (solid line).

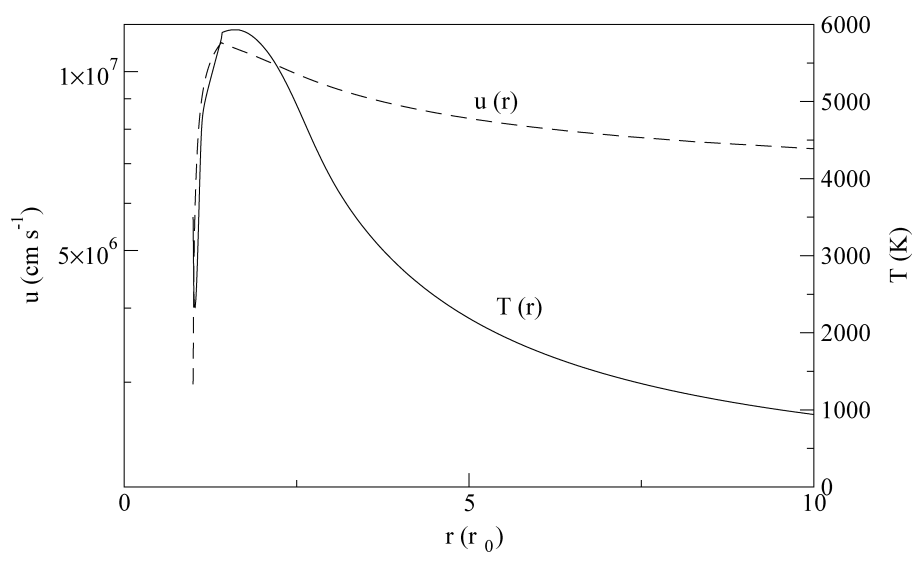

Figure 2. The wind velocity profile and the wind temperature profile for the best fitting parameters in the case of low damped waves.

and it slightly decreases for larger distances until reaching the observed value. The temperature profile presents an initial negative gradient reaching temperatures $T<2500 \mathrm{~K}$ in a narrow region. Also, near $r=1.5 r_{0}$ the temperature reaches the maximum value of $\sim 6000 \mathrm{~K}$. For $r>3.0 r_{0}$, where the wave heating and the radiative losses are low, the temperature decreases due to the adiabatic expansion.

\section{Conclusions}

We propose a self-consistent wind model to determine the parameters profiles for a supergiant late-type star. To determine the magnetic field geometry we used an expansion method over the wind physical parameters as proposed by Pneuman 
et al. (1986). Near the surface, the magnetic pressure inside the flux tubes is higher than that of the surrounding medium, forcing the field lines to curve. We found an initial super-radial expansion factor $S>30$ at the wind basis, much higher than the value used by previous authors (e.g., Jatenco-Pereira and Opher, 1989; Bravo and Stewart, 1997; Dobrzycka et al., 1999) that included empirical relations to account for the magnetic field geometry based on solar observations.

Considering a supergiant late-type star, we obtained the wind velocity and temperature profiles. Typically, Alfvén waves driven winds result in high velocity winds $\left(u>100 \mathrm{~km} \mathrm{~s}^{-1}\right.$ ), unless some strong wave-damping mechanism takes place at the wind basis. We showed that this conclusion is correct in the case of low divergent magnetic field structures. In this work, the strong divergence is responsible for a rapid wave spatial dilution near surface, resulting in a lower wind velocity even for low damped waves.

The obtained results were consistent to the typical mass-loss rate $(\dot{M} \simeq$ $10^{-7}$ to $\left.10^{-6} \mathrm{M}_{\odot} \mathrm{yr}^{-1}\right)$ and the terminal velocity $\left(u \simeq 70 \mathrm{~km} \mathrm{~s}^{-1}\right)$ observed for these objects. The velocity profile reveals an efficient acceleration at $r<1.5 r_{0}$, reaching the maximum value $\sim 100 \mathrm{~km} \mathrm{~s}^{-1}$. In this region the wind is mainly accelerated by the wave energy density and the thermal pressure gradients. Afterwards, the absence of the wave acceleration and the cooling gas result in a decrease of the velocity to the observed values. For the temperature, assuming a weakly damped wave flux, the radiative losses and the expansion cooling are dominant near the surface, and the temperature gradient is initially negative. The temperature falls to $\sim 2500 \mathrm{~K}$ near the stellar surface. Afterwards, as the density decreases due to the wind acceleration and flux tube expansion, the temperature increases up to $\sim 6000 \mathrm{~K}$ at $r<2 r_{0}$. For higher distances, where the radiative losses are low and the wave heating is no longer effective, the temperature decreases mainly due to the adiabatic expansion.

Although the present calculations provided a new and interesting picture of the physical processes involving the heating and the acceleration mechanisms of cool stellar winds, the model presents a limitation. The wind equations were solved considering the thin flux tube approximation. Mainly for a high-divergence wind, a complete 2D set of equations must be employed in order to obtain more precise results. This step shall be concluded in the near future.

\section{Acknowledgements}

A.A. Vidotto and D. Falceta-Gonçalves thank FAPESP for the finantial support (04/13846-6 and 04/12053-2) and V. Jatenco-Pereira thanks CNPq (304523/90-9).

\section{References}

Airapetian, V.S., Carpenter, K., and Ofman, L.: 2003, AAS 202, 3214.

Bravo, S., and Stewart, G.A.: 1997, AdSpR 20, 35. 
Dobrzycka, D., Cranmer, S.R., Panasyuk, A.V., Strachan, L., and Kohl, J. L.: 1999, JGR 104, 9791. Dupree, A.K.: 1986, ARA\&A 24, 377.

Guandalini, R., Busso, M., Ciprini, S., Silvestro, G., and Persi, P.: 2006, A\&A 445, 1069.

Hartmann, L., and MacGregor, K.B.: 1980, ApJ 242, 260.

Hartmann, L., Edwards, S., and Avrett, E.: 1982, ApJ 261, 279.

Jatenco-Pereira, V., and Opher, R.: 1989, A\&A 209, 327.

Lamers, H.J.G.L.M., and Cassinelli, J.P.: 1999, Introduction to Stellar Winds, Cambridge University Press, New York.

Lagage, P.O., and Cesarsky, C.J.: 1983, A\&A 125, 249.

Longcope, D.W., and Klapper, I.: 1997, ApJ 488, 443.

Pneuman, G.W., Solanki, S.K., and Stenflo, J.O.: 1986, A\&A 154, 231.

Sandin, C., and Hofner, S.: 2003, A\&A 404, 789.

Schmutzler, T., and Tscharnuter, W.M.: 1993, A\&A 273, 318.

Spruit, H.C.: 1981, A\&A 98, 155.

Suzuki, T.K., and Inutsuka, S.: 2005, ApJL 632, 49.

Usmanov, A.V., Goldstein, M.L., Besser, B.P., and Fritzer, J.M.: 2000, JGR 105, 12675. 


\title{
On the magnetic structure and wind parameter profiles of Alfvén wave driven winds in late-type supergiant stars
}

\author{
D. Falceta-Gonçalves, ${ }^{\star}$ A. A. Vidotto and V. Jatenco-Pereira \\ Instituto de Astronomia, Geofí sica e Ciências Atmosféricas, Universidade de São Paulo, Rua do Matão 1226, CEP 05508-900, São Paulo, Brazil
}

Accepted 2006 February 10. Received 2006 February 9; in original form 2005 October 27

\begin{abstract}
Cool stars at giant and supergiant evolutionary phases present low-velocity and high-density winds, responsible for the observed high mass-loss rates. Although presenting high luminosities, radiation pressure on dust particles is not sufficient to explain the wind acceleration process. Among the possible solutions to this still unsolved problem, Alfvén waves are, probably, the most interesting for their high efficiency in transfering energy and momentum to the wind. Typically, models of Alfvén wave driven winds result in high-velocity winds if they are not highly damped. In this work, we determine self-consistently the magnetic field geometry and solve the momentum, energy and mass conservation equations, to demonstrate that even a low-damped Alfvén wave flux is able to reproduce the low-velocity wind. We show that the magnetic flux tubes expand with a super-radial factor of $S>30$ near the stellar surface, larger than that used in previous semi-empirical models. The rapid expansion results in a strong spatial dilution of the wave flux. We obtained the wind parameter profiles for a typical supergiant star of $16 \mathrm{M}_{\odot}$. The wind is accelerated in a narrow region, coincident with the region of high divergence of the magnetic field lines, up to $100 \mathrm{~km} \mathrm{~s}^{-1}$. For the temperature, we obtained a slight decrease near the surface for low-damped waves, because the wave heating mechanism is less effective than the radiative losses. The peak temperature occurs at $r \simeq 1.5 r_{0}$ reaching $6000 \mathrm{~K}$. Propagating outwards, the wind cools down mainly due to adiabatic expansion.
\end{abstract}

Key words: MHD - waves - stars: magnetic fields - stars: mass-loss.

\section{INTRODUCTION}

Cool giant and supergiant stars are known to present continuous mass-loss process occuring at high rates, typically $10^{-10}$ $10^{-5} \mathrm{M}_{\odot} \mathrm{yr}^{-1}$, but in low-velocity winds $\left(u_{\infty}<300 \mathrm{~km} \mathrm{~s}^{-1}\right)$ (Dupree 1986; Lamers \& Cassinelli 1999). After decades of theoretical and observational studies, the mechanisms in which the wind acceleration occurs are still poorly understood. Due to the stellar high luminosity and low effective temperature, several authors have proposed radiativelly dust-driven models to explain the observed wind properties (Elitzur \& Ivezic 2001; Liberatore, Lafon $\&$ Berruyer 2001; Woitke \& Niccolini 2005). At the pulsating phase, acoustic waves generate high-density shells that could allow dust to form near the stellar surface. In this scenario, radiative pressure on grains transfers momentum to these particles being responsible for their acceleration and, if gas and dust are dynamically well coupled, grains drag the gas outwards resulting in the mass ejection. However, at stationary envelopes [e.g. pre-asymptotic giant branch (pre-AGB) phase] dust-driven theoretical models have lately failed in reproducing the wind properties, mainly because the dust-gas coupling is not

^E-mail: diego@astro.iag.usp.br effective (Sandin \& Hofner 2003). Observationally, Guandalini et al. (2006) found no strong correlation between the mass-loss rates and the luminosities of AGB stars. Their main conclusion is that, if radiative pressure is important in powering these stellar winds, it must occur in addition to other mechanism. Another drawback to the radiation pressure models is the need for the dust-formation region to be close to the star. Recent high-resolution Doppler measurements show that winds are mainly accelerated near the stellar surface $\left(r<1.3 R_{*}\right)$ (Airapetian, Carpenter \& Ofman 2003), while grains are expected to grow and survive at even larger distances.

In this sense, another mechanism must be used to accelerate the gas near surface. The most promising mechanism for the winds of cool stars is the transfer of momentum and energy to the wind from magnetohydrodynamics (MHD) waves. Alfvén waves driven wind models are known to result in high-velocity winds, unlike what is measured for giant cool stars, because these MHD waves are, in general, weakly damped. Hartmann \& MacGregor (1980) showed that it would be possible to reproduce the observed low-wind velocities and the high mass-loss rates of the cool giant and supergiant stars if some kind of wave damping mechanism is effective at the wind basis $\left(r<2 R_{*}\right)$. In that work, they confirmed this assumption using the ion-friction damping, which has a damping length proportional to $P^{2}$, where $P$ is the waveperiod. Holzer, Fla $\&$ Leer 


\section{D. Falceta-Gonçalves, A. A. Vidotto and V. Jatenco-Pereira}

(1983) questioned this result in terms of an unnatural fine-tuning for the wave flux period, and argued that stars would, more reasonably, present a wide variety of wavefrequencies depending on the generation mechanisms, and for most of them the damping would be uneffective. However, Jatenco-Pereira \& Opher (1989) studied the effects of both different damping mechanisms and the magnetic field divergence, showing that the fine-tuning of the waveperiod is unnecessary since there are several other damping mechanisms that would act on the frequency spectrum. Also, they showed that the magnetic field divergent geometry can rapidly dilute the wave flux and also slow down the wind. Their magnetic field geometry was based on the empirical relations found from observations of the solar wind. This because (in general) in lack of direct measurements of the magnetic field fluctuations and structure in other stars, we have to simply extrapolate our knowledge from the solar observations.

Parker (1958) proposed that the solar wind is accelerated by the strong thermal pressure gradient in the transition region, where the gas temperature increases from $\sim 10^{4}$ up to $10^{6} \mathrm{~K}$. However, it became clear in the following years that neither the solar radiation nor the acoustic waves generated in the photosphere, could account for the heating of the coronal base. The origin of the energy responsible for the heating and acceleration of the plasma is believed to be from both magnetic field reconnections above the photosphere (Axford \& McKenzie 1996) and convective motions under the stellar surface. The convective energy is transferred up to the atmosphere from the perturbations generated in the field-line footpoints (Cranmer \& van Ballegooijen 2005). These perturbations propagate outwards as Alfvén waves, heating the gas as they are damped (Suzuki \& Inutsuka 2005). Regarding the magnetic field lines, Holzer \& Leer (1980) and Jatenco-Pereira \& Opher (1989) realized that the superradial geometry of the magnetic field funnels at the coronal holes could have a significant impact on the mass flux and wind speed. Esser et al. (2005) showed that the models considering highly diverging magnetic funnels explain better the observed data for the Sun. Also, Tu et al. (2005) established that the solar wind acceleration initiates in the magnetic funnels at heights lower than $2 \times 10^{9} \mathrm{~cm}$, coincident with the high-divergence region.

In this work, we model the acceleration and heating of a late-type supergiant stellar wind considering an outward flux of Alfvén waves. We solve the MHD equations to, self-consistently, determine the magnetic field geometry and the wind temperature, density and velocity profiles. In Section 2, we describe the model basic equations. In Section 3, we present the results and the discussions, followed, by the conclusions of this work.

\section{THE MODEL}

The wind equations are based on mass, momentum, energy and magnetic flux conservation. The first is given by:

$\rho u A(r)=\rho_{0} u_{0} A\left(r_{0}\right)$,

where $u$ is the flow velocity, $\rho$ is the gas density and $A(r)$ is the flow cross-section area at a distance $r$ from the centre of the star. The index ' 0 ' indicates the variable is being evaluated at the stellar surface $\left(r=r_{0}\right)$.

Assuming a steady flow, the momentum equation can be written as:

$$
\begin{aligned}
\rho(\boldsymbol{u} \cdot \nabla) \boldsymbol{u}= & -\rho \frac{G M_{*}}{r^{3}} \boldsymbol{r}-\nabla P-\nabla\left(\frac{\left\langle(\delta B)^{2}\right\rangle}{8 \pi}\right) \\
& +\frac{1}{4 \pi}(\boldsymbol{B} \cdot \nabla) \boldsymbol{B}-\nabla\left(\frac{B^{2}}{8 \pi}\right),
\end{aligned}
$$

where $P=\rho k_{\mathrm{B}} T / m$ is the thermal pressure, $k_{\mathrm{B}}$ is the Boltzmann constant, $T$ is the gas temperature, $m$ is the mean mass per particle, $G$ the gravitational constant and $\delta B$ the wave magnetic field amplitude. In equation (2), the right-hand side contains the gravitational force density and the thermal and wave pressure gradients, respectively. The last two terms represent the Lorentz force. The wave amplitude $(\delta B)$ is related to the wave energy density $(\epsilon)$ by $\epsilon=\left\langle(\delta B)^{2}\right\rangle /(4 \pi)$.

\subsection{Thin flux tube approximation}

Typically, considering magnetic field strengths $>1 \mathrm{G}$, the wind basis is characterized by the relation $B^{2} / 8 \pi \gg P>\rho u^{2} / 2$, that is, the plasma is magnetically dominated. In this case, if we assume the wind to be initiated at funnels anchored at the stellar surface, which are surrounded by a plasma with lower magnetic field strength, the magnetic pressure inside will push the gas and the funnel field lines will expand. The funnel cross-section radius $(\mathcal{R})$ will grow superradially up to a limit value $\left(\mathcal{R}_{\mathrm{m}}\right)$. This limiting cross-section radius depends both on the relation between external and internal magnetic field strengths and on the filling factor $(\alpha)$. As the area increases, the internal magnetic strength diminishes until the equilibrium between internal and external magnetic pressures is reached. If the internal magnetic field strength is much larger than the external, the flux tubes cross-section will depend on the filling factor only. The filling factor is the ratio between the area of the stellar surface covered by funnels and the total area. The averaged maximum area that a funnel could reach would be $A_{\mathrm{m}}=A\left(r_{0}\right) / \alpha$ or, in terms of the cross-section radius:

$$
\mathcal{R}_{\mathrm{m}}=\frac{\mathcal{R}_{0}}{\alpha^{1 / 2}} .
$$

For the quiet Sun, the funnels that merge to form the coronal holes cover about 10 per cent of the total surface.

To evaluate the tube expansion at the wind basis, we assume the plasma to be magnetically dominated and the left-hand side of equation (2) may be neglected if compared to the other terms. Then, by using $\nabla \cdot \boldsymbol{B}=0$ and a power series expansion method proposed by Pneuman, Solanki \& Stenflo (1986), we can determine self-consistently the magnetic field geometry without assuming any empirical function for the funnel cross-section with distance.

Following Pneuman et al. (1986), using the thin flux tube aproximation, Equation (2) and $\nabla \cdot \boldsymbol{B}=0$ are described near stellar surface by:

$4 \pi \frac{\partial P}{\partial y} \simeq B_{r}\left(\frac{\partial B_{y}}{\partial r}-\frac{\partial B_{r}}{\partial y}\right)$,

$4 \pi\left(\frac{\partial P}{\partial r}+\frac{P}{H}\right) \simeq-B_{y}\left(\frac{\partial B_{y}}{\partial r}-\frac{\partial B_{r}}{\partial y}\right)$

and

$\frac{1}{y} \frac{\partial}{\partial y}\left(y B_{y}\right)+\frac{\partial B_{r}}{\partial r}=0$

where $H=k_{\mathrm{B}} T r^{2} / G m M_{*}$ is the scaleheight.

Expanding all variables as power series in $y$ (i.e. along the tube radius), and neglecting terms of orders higher than 2 , equations (4)-(6) give rise to a differential equation for the flux tube cross-section:

$$
\begin{aligned}
& \frac{A\left(r_{0}\right)}{2 H_{0}^{2}}\left\{\frac{\partial^{2}}{\partial r^{2}}\left[\frac{A\left(r_{0}\right)}{A(r)}\right]-\frac{1}{2 A(r)} \frac{\partial}{\partial r}\left[\frac{A\left(r_{0}\right)}{A(r)}\right]\right\} \\
& =\left[\frac{A\left(r_{0}\right)}{A(r)}\right]^{2}\left\{1-\left[\frac{B_{\text {ext }}}{B_{0}} \frac{(1-\alpha)}{\left[A\left(r_{0}\right) / A(r)\right]-\alpha}\right]^{2}\right\}+2 \beta \frac{P(r)}{P\left(r_{0}\right)},
\end{aligned}
$$


The structure of Alfvén wave driven winds

1147

where $\beta=4 \pi P\left(r_{0}\right) / B^{2}\left(r_{0}\right), \alpha$ is the filling factor and $B_{\mathrm{ext}}$ is the magnetic field strength outside the flux tube. In the following calculations, we fixed its value to be $10^{-3} B(r)$.

To simplify the set of equations, we will define the funnel area expansion as a function of radial distance by:

$A(r)=A\left(r_{0}\right)\left(\frac{r}{r_{0}}\right)^{S}$,

where $S$ is the expansion index, which is super-radial $(\mathrm{S}>2)$ at the wind basis up to the merging radius when $S$ becomes $2 . S$ is determined from equation (7).

\subsection{The energy equation}

Falceta-Gonç \& Jatenco-Pereira (2002) showed that, even though presenting low effective temperatures, the temperature gradients at the wind basis of late-type supergiant stars could play an important role in accelerating the gas, as occurs in the Sun. In their model, the temperature profile was assumed to be a $r$-dependent function, obtained from observational data. The wind temperatures in giant cool stars are expected to increase from the photosferic value up to $10^{4} \mathrm{~K}$, much lower than what is observed for the Sun, but still important in the wind acceleration if the gradient occurs in small length-scales. From their calculations, Falceta-Gonç \& Jatenco-Pereira (2002) also showed that the thermal pressure is even more important than radiation pressure at the non-pulsating phase of these objects.

In a consistent model, to avoid assuming any empirical function for the magnetic field geometry and to determine the wind temperature at each wind position $(r)$, we have to solve the energy equation, which is determined from the balance between wave heating and the adiabatic expansion and radiative coolings (Hartmann, Edwards \& Avrett 1982; Vidotto \& Jatenco-Pereira 2006). Thus, neglecting conduction, we write the energy equation as:

$\rho u \frac{\mathrm{d}}{\mathrm{d} r}\left(\frac{u^{2}}{2}+\frac{5}{2} \frac{k_{\mathrm{B}} T}{m}-\frac{G M_{\star}}{r}\right)+\frac{u}{2} \frac{\mathrm{d} \epsilon}{\mathrm{d} r}=\left(Q-P_{\mathrm{R}}\right)$,

where $\epsilon=\left\langle(\delta B)^{2}\right\rangle /(4 \pi)$ is the wave energy density, which is described below (Section 2.3), $Q$ is the wave heating rate, that is, the rate at which the gas is being heated due to dissipation of wave energy, and $P_{\mathrm{R}}$ is the radiative cooling rate, both in $\mathrm{erg} \mathrm{cm}^{-3} \mathrm{~s}^{-1}$. The wave heating can be written as:

$Q=\frac{\epsilon}{L}\left(u+v_{\mathrm{A}}\right)$

and the radiative cooling is given by:

$P_{\mathrm{R}}=\Lambda n_{\mathrm{e}} n_{\mathrm{H}}$,

where $n_{\mathrm{e}}$ is the electron density, $n_{\mathrm{H}}$ is the hydrogen density and $\Lambda$ is the radiative loss function. Here, we adopt the $\Lambda$ function given by Schmutzler \& Tscharnuter (1993) and calculate $n_{\mathrm{e}}$ with the modified Saha equation given by Hartmann \& MacGregor (1980).

\subsection{Wave energy density}

The wave energy density at each step may be calculated, using a WKB aproximation, from the wave action conservation. Under this assumption, the wave energy density is dissipated as follows:

$\epsilon=\epsilon_{0} \frac{M_{0}}{M}\left(\frac{1+M_{0}}{1+M}\right)^{2} \exp \left(-\int_{r_{0}}^{r} \frac{1}{L} \mathrm{~d} r^{\prime}\right)$,

where $M=u / v_{\mathrm{A}}$ is the Alfvén-Mach number, $v_{\mathrm{A}}=(B / \sqrt{4 \pi \rho})$ the Alfvén speed and $L$ the wave damping length. Also, the wave flux $\left(\phi_{\mathrm{A}}\right)$ at $r_{0}$ is evaluated by (Jatenco-Pereira \& Opher 1989):

$\phi_{\mathrm{A} 0}=\epsilon_{0} v_{\mathrm{A} 0}\left(1+\frac{3}{2} M_{0}\right)$.

Actually, the WKB aproximation is not valid when the perturbation wavelength is much larger than the gradients length-scales of the system, for example, when $\lambda>L$. As a consequence, part of the wave flux is reflected and the Alfvén waves propagating on opposite directions decay as the generated beat waves interact with the gas particles. Davila (1985) pointed out that, in the Sun, the gradients of the coronal parameters are high enough to make the WKB aproximation not applicable. However, Usmanov et al. (2000) found good agreement between the observational data and models with this approach. In this model, we used equation (12) assuming then linear perturbations, however, included non-linear effects in the damping length function $\left(L_{\mathrm{NL}}\right)$. The non-linear damping length is given by (Jatenco-Pereira \& Opher 1989):

$L_{N L}=L_{0}\left(\frac{v_{\mathrm{A}}}{v_{\mathrm{A} 0}}\right)^{4} \frac{\left\langle(\delta v)^{2}\right\rangle_{0}}{\left\langle(\delta v)^{2}\right\rangle}(1+M)$,

where $\left\langle(\delta v)^{2}\right\rangle$ is the averaged-squared perturbation velocity amplitude and $L_{0}$ is the damping length at the wind basis, which is mainly dependent on the assumed wavefrequency spectrum (Lagage \& Cesarsky 1983). Here, we will let it as a free parameter.

\subsection{The wind equations}

Finally, once the magnetic field structure is determined, we evaluate the temperature and velocity of the wind along the magnetic field. Using the equation (8) and considering the magnetic flux conservation, equations (1), (2) and (9) in the radial direction are reduced to (Vidotto \& Jatenco-Pereira 2006):

$\frac{\mathrm{d} T}{\mathrm{~d} r}=\frac{2}{3} \frac{T}{r}\left[\frac{r\left(Q-P_{\mathrm{R}}\right)}{\rho u\left(k_{\mathrm{B}} T / m\right)}-\left(S+\frac{r}{u} \frac{\mathrm{d} u}{\mathrm{~d} r}\right)\right]$

and

$$
\begin{gathered}
\frac{1}{u} \frac{\mathrm{d} u}{\mathrm{~d} r}\left[u^{2}-\frac{5}{3} \frac{k_{\mathrm{B}} T}{m}-\frac{\left\langle(\delta v)^{2}\right\rangle}{4}\left(\frac{1+3 M}{1+M}\right)\right] \\
=\frac{S}{r}\left[\frac{5}{3} \frac{k_{\mathrm{B}} T}{m}-\frac{2}{3} \frac{r\left(Q-P_{\mathrm{R}}\right)}{S \rho u}-\frac{G M_{\star}}{r S}\right. \\
\left.+\frac{\left\langle(\delta v)^{2}\right\rangle}{2 L S} r+\frac{\left\langle(\delta v)^{2}\right\rangle}{4}\left(\frac{1+3 M}{1+M}\right)\right] .
\end{gathered}
$$

Equations (13)-(16) fully describe the wind parameters and the magnetic field geometry under the given assumptions. In Section 3, we show the main results by applying these equations in a typical cool supergiant star and compare them with previous works.

\section{RESULTS AND DISCUSSIONS}

For the last decades, the validity of a wind model was constrained in reproducing only both the terminal velocity and the mass-loss rate of a given star. As a consequence, a number of accelerating mechanisms were found in accordance to observations of these constrains. However, with the high-resolution observations and more sensitive intruments, the parameters radial profiles will become measurable and will be decisive on the modelling choice. Here, we determine the velocity, density and temperature profiles and discuss their dependence on the initial assumptions. 


\section{D. Falceta-Gonçalves, A. A. Vidotto and V. Jatenco-Pereira}

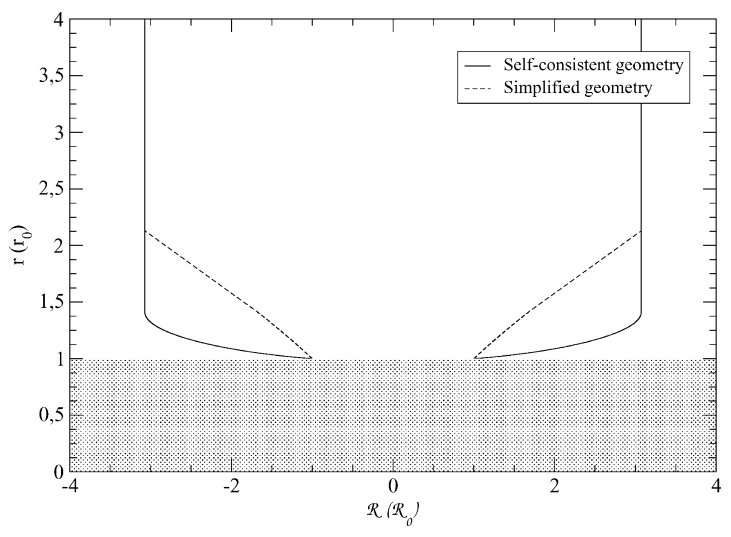

Figure 1. The magnetic field structure for a constant super-radial index $S=5$ (dashed line) and that determined self-consistently in our model (solid line).

We applied the described model on a cool supergiant star with $M_{*}=16 \mathrm{M}_{\odot}, r_{0}=400 \mathrm{R}_{\odot}, \rho_{0}=10^{-13} \mathrm{~g} \mathrm{~cm}^{-3}, B_{0}=10 \mathrm{G}$ and $T_{0}=3500 \mathrm{~K}$. We also assumed a filling factor $\alpha=0.1$, according to the solar observations.

\subsection{Wind profiles}

To study the importance of the damping length on the wind profiles, we performed calculations setting the initial wave flux as $\phi_{\mathrm{A} 0}=$ $3.10^{7} \mathrm{erg} \mathrm{cm}^{-2} \mathrm{~s}^{-1}$, and varied $L_{0}$ as $0.2,1.0$ and $5.0 r_{0}$. The magnetic field structure is shown in Fig. 1. The solid line represents the solution of equation (7) for the given stellar parameters, compared to a simple constant expansion factor $S=5$ (dashed line), as used by Falceta-Gonç \& Jatenco-Pereira (2002). Comparing both curves, we find that the self-consistent geometry is more divergent near stellar surface, and the flux tubes are expected to merge at lower distances, in agreement with more refined models for the Sun (Esser et al. 2005). We obtained an initial value of $S\left(r_{0}\right) \simeq 35$, that decreases with distance to the stellar surface. From the assumed $\alpha$, we found that the neighbour flux tubes merge at $r=1.41 r_{0}$. At this point, with a sudden decrease of $S$ from its super-radial value to $S=2$ (radial), the wind properties change and it is notable as breaks in the temperature, density and velocity profiles.

We obtained the same geometry for the following calculations considering different initial parameters. This is because the superradial magnetic field geometry occurs mainly at the wind basis, where the magnetic pressure is highly dominant. In this sense, we expect the expansion index $(S)$ to be independent of the other initial parameters such as the wind velocity, the wave flux densities and the damping length.

In Fig. 2, we compare the wind velocity profiles for the different initial damping lengths. As expected, the larger the damping length the higher is the wind velocity. However, for previous works that do not take into account any high initial divergence, the wind velocity is even larger. The obtained divergence result in a fast dilution of the wave energy density near the stellar surface. As a consequence, the lack of wave flux at larger distances results in lower velocities. This result shows that even low-damped wave fluxes can be responsible for low terminal velocities, depending on the field divergence.

In Figs 3-5, we present the gas mass density, wave energy density and the temperature, respectively, for the same calculations. For the

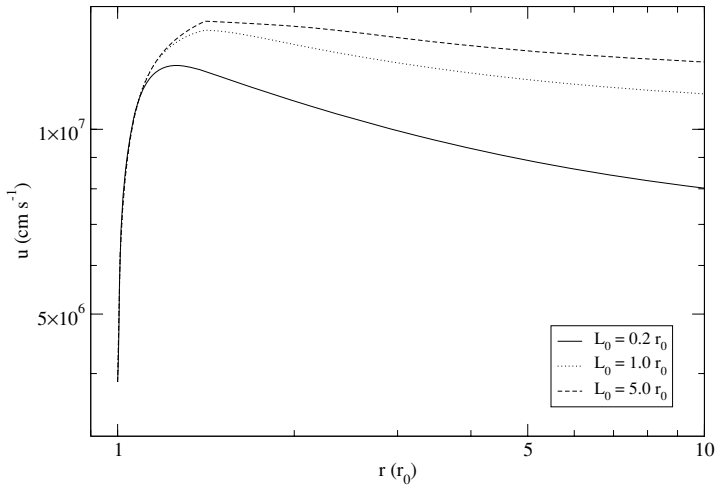

Figure 2. Wind velocity profiles obtained for the initial damping lengths $L=0.2 r_{0}$ (solid line), $L=1.0 r_{0}$ (dotted line) and $L=5.0 r_{0}$ (dashed line) as function of the distance.

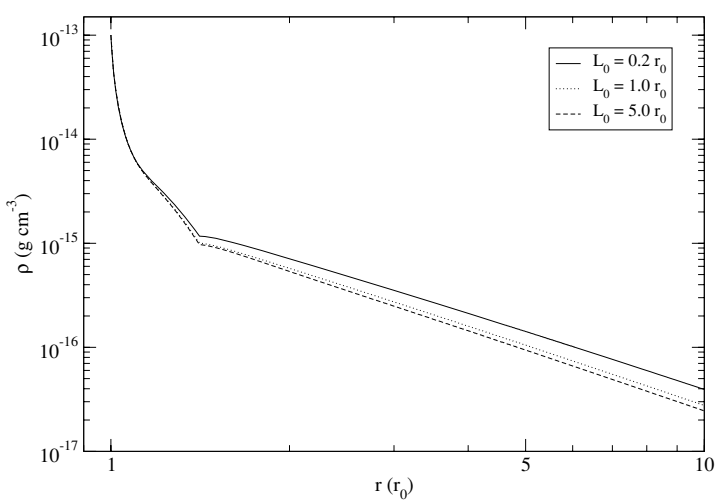

Figure 3. Wind density profiles obtained for the initial damping length $L=0.2 r_{0}$ (solid line), $L=1.0 r_{0}$ (dotted line) and $L=5.0 r_{0}$ (dashed line) as function of the distance.

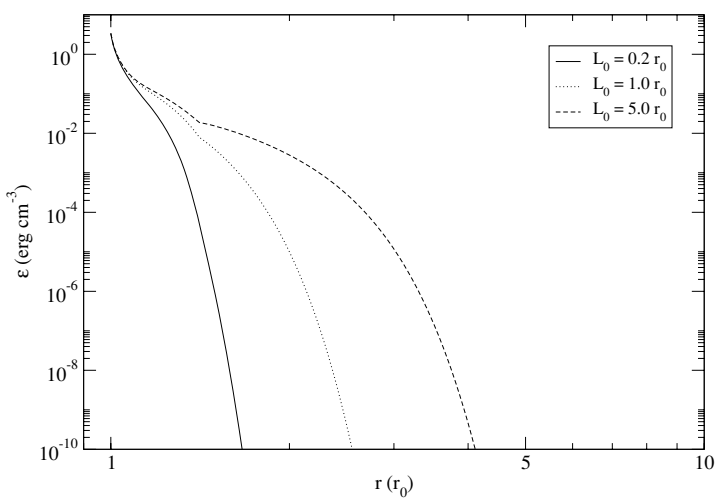

Figure 4. Wave energy density profiles obtained for the initial damping lengths $L=0.2 r_{0}$ (solid line), $L=1.0 r_{0}$ (dotted line) and $L=5.0 r_{0}$ (dashed line) as function of the distance. 
The structure of Alfvén wave driven winds

1149

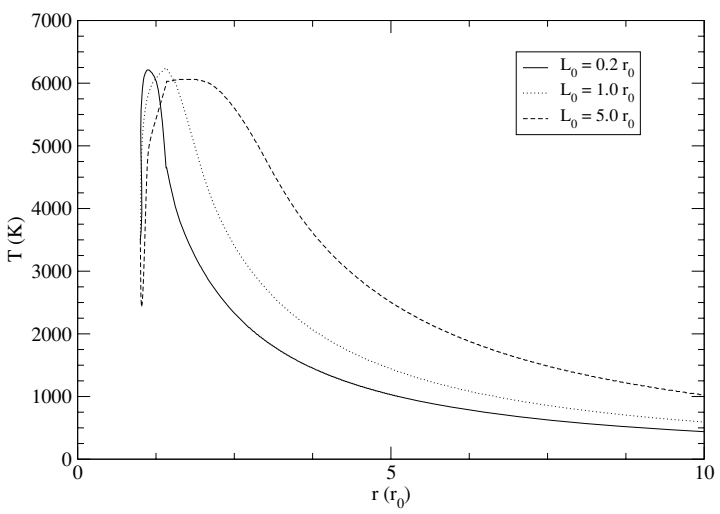

Figure 5. Wind temperature profiles obtained for the initial damping lengths $L=0.2 r_{0}$ (solid line), $L=1.0 r_{0}$ (dotted line) and $L=5.0 r_{0}$ (dashed line) as function of the distance.

gas density, all three lines show the same behaviour at $r<1.5 r_{0}$ indicating that the flux tube divergence is the dominant factor if comparing to the gas expansion due to acceleration. This is an interesting result since, typically, the density profile is believed to be closely related to the velocity profile only. In Fig. 4, we note that, in despite of what was obtained in previous works, the wave energy density is weakly dependent on the initial damping length at the wind basis. This mainly because, again, the field divergence is dominant on the dilution process. On the other hand, for distances larger than the flux tubes merging position, the wave damping is dominant. In Fig. 5, the temperature profiles show different features. For the given stellar parameters, if $L_{0}=5.0 r_{0}$, the wave heating is less effective and the radiative losses added to the expansion cooling result in a negative temperature gradient, which occurs in a very narrow region until the density becomes low enough for the radiative losses to become less important. For highly damped waves, the wave heating dominates the radiative losses and the temperature gradient is positive near the surface. Another interesting feature is the maximum temperature position. Since the temperature gradient is mainly dependent on the wave damping (via $Q$ parameter), we note that the lower is the damping length, the closer to the stellar surface the maximum temperature occurs.

Recently, Zeeman splitting of $\mathrm{H}_{2} \mathrm{O}$ masers observations led Vlemmings, Diamond \& van Lengevelde (2002) to infer magnetic field strengths on supergiant stellar surfaces of $B_{0}>100 \mathrm{G}$. Also, theoretical dynamo models for AGB stars reveal that the planetary nebulae shapes can be explained by the magnetic field strengths of $\sim 200 \mathrm{G}$ (Blackman et al. 2001). For this reason, we also perfomed numerical calculations for $B_{0}=10$ and $100 \mathrm{G}$, setting the initial parameters for a low-damped wave flux $\left(L_{0}=5 r_{0}\right)$. We also assumed the same wave amplitude relative to the magnetic field for both cases.

The velocity profile for both initial magnetic field strengths are shown in Fig. 6. For the larger magnetic field strength $\left(B_{0}=\right.$ $100 \mathrm{G})$, we obtained the higher terminal velocity $\left(u>200 \mathrm{~km} \mathrm{~s}^{-1}\right)$. This effect is mainly due to the higher wave energy flux assumed at the wind basis to accomplish the same relative wave amplitude.

The temperature profiles, shown in Fig. 7, also present features dependent on the magnetic field strength. As for the velocity, a high wave energy flux results in a high temperature, at least near the stellar surface due to the higher energy density.

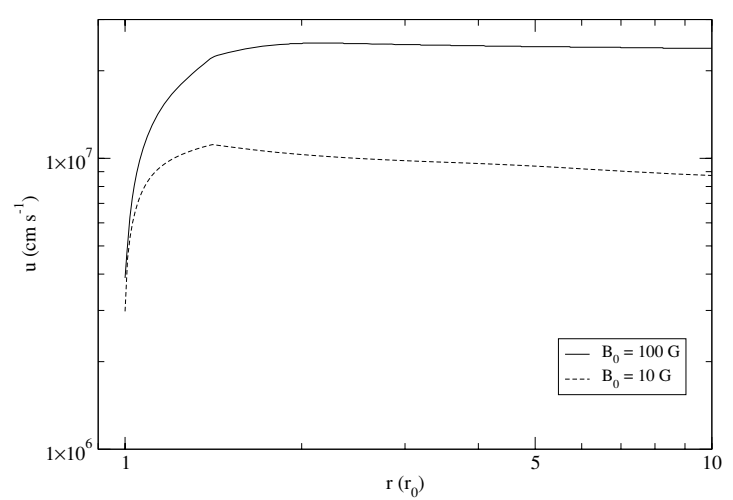

Figure 6. Wind velocity profiles obtained for the initial magnetic field strengths $B_{0}=10 \mathrm{G}$ (dotted line) and $B_{0}=100 \mathrm{G}$ (solid line) as function of the distance.

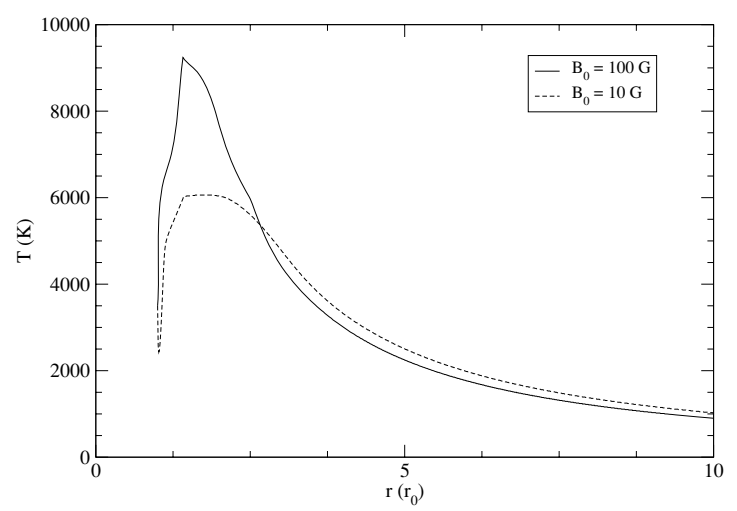

Figure 7. Wind temperature profiles obtained for the initial magnetic field strengths $B_{0}=10 \mathrm{G}$ (dotted line) and $B_{0}=100 \mathrm{G}$ (solid line) as function of the distance.

\subsection{Best-fitting model}

For stars with parameters similar to those used in the previous subsection, observational data reveal typical mass-loss rates of $\dot{M} \simeq$ $10^{-7}$ to $10^{-6} \mathrm{M}_{\odot} \mathrm{yr}^{-1}$ and terminal velocities of $u \simeq 70 \mathrm{~km} \mathrm{~s}^{-1}$. Unfortunately, the available data are limited in spatial resolution for most of the stars, and it is not possible to fit the complete radial profiles.

Assuming a surface magnetic field strength $B_{0}=10 \mathrm{G}$, and a low-damped wave flux $\left(L_{0}=5 r_{0}\right)$, it was possible to reproduce both the wind terminal velocity and the mass-loss rate using an Alfvén waves flux of $\phi_{\mathrm{A} 0}=10^{7} \mathrm{erg} \mathrm{cm}^{-2} \mathrm{~s}^{-1}$ at the wind basis. This value corresponds to a wave amplitude of $\left\langle(\delta B)^{2}\right\rangle^{1 / 2} \simeq 3 \times$ $10^{-2} B_{0}$, which is very plausible for a turbulent medium as that at the stellar surface.

The velocity and temperature profiles for this case are shown in Figs 8 and 9, respectively. The velocity profile reveals a peak of $u>100 \mathrm{~km} \mathrm{~s}^{-1}$ at $r<2.0 r_{0}$, and slightly decreases for larger distances until reaching the observed value. The temperature profile presents an initial negative gradient reaching temperatures $T<$ $2500 \mathrm{~K}$ in a narrow region. Also, near $r=1.5 r_{0}$, the temperature reaches the maximum value of $\sim 6000 \mathrm{~K}$. For $r>3.0 r_{0}$, where 


\section{D. Falceta-Gonçalves, A. A. Vidotto and V. Jatenco-Pereira}

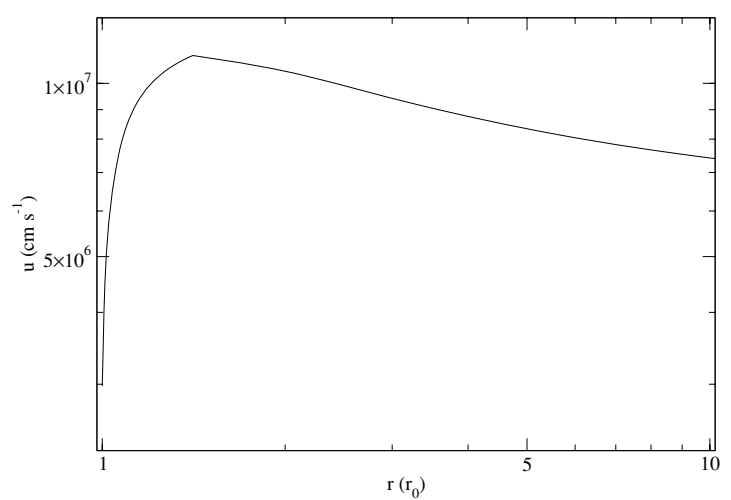

Figure 8. The wind velocity profile for the best-fitting parameters in the case of low-damped waves. Here, we assumed $B_{0}=10 \mathrm{G}, L_{0}=5.0 r_{0}$ and $\phi_{\mathrm{A} 0}=10^{7} \mathrm{erg} \mathrm{cm}^{-2} \mathrm{~s}^{-1}$

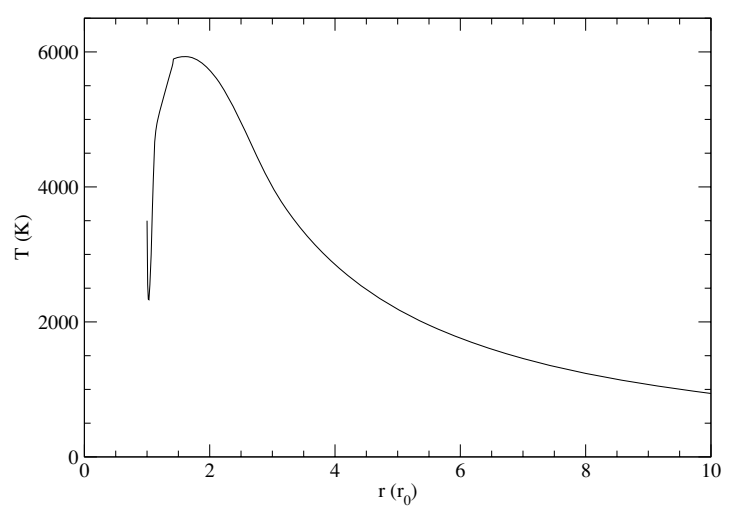

Figure 9. The wind temperature profile for the best-fitting parameters in the case of low-damped waves. Here, we assumed $B_{0}=10 \mathrm{G}, L_{0}=5.0 r_{0}$ and $\phi_{\mathrm{A} 0}=10^{7} \mathrm{erg} \mathrm{cm}^{-2} \mathrm{~s}^{-1}$.

the wave heating and the radiative losses are low, the temperature decreases due to the adiabatic expansion.

\section{CONCLUSIONS}

We propose a self-consistent wind model to determine the parameters profiles for a supergiant late-type star. To determine the magnetic field geometry, we used an expansion method over the wind physical parameters as proposed by Pneuman et al. (1986). Near surface, the magnetic pressure inside the flux tubes are higher than that of the surrounding medium, forcing the field lines to curve. We found an initial super-radial expansion factor $S>30$ at the wind basis, much higher than the value used by previous authors that included empirical relations to account for the magnetic field geometry based on the solar observations.

Considering a supergiant late-type star with $M_{*}=16 \mathrm{M}_{\odot}$, $r_{0}=400 \mathrm{R}_{\odot}, \rho_{0}=10^{-13} \mathrm{~g} \mathrm{~cm}^{-3}, B_{0}=10 \mathrm{G}$ and $T_{0}=3500 \mathrm{~K}$, we obtained the wind velocity, density and temperature profiles. Typically, Alfvén wave driven winds result in high-velocity winds $\left(u>100 \mathrm{~km} \mathrm{~s}^{-1}\right)$, unless some strong wave damping mechanism takes place at the wind basis. We showed that this conclusion is correct in the case of low-divergent magnetic field structures. In this work, the strong divergence is responsible for a rapid wave spatial dilution near surface, resulting in a lower wind velocity even for low-damped waves.

We reproduced both the typical mass-loss rate $\left(\dot{M} \simeq 10^{-7}\right.$ to $\left.10^{-6} \mathrm{M}_{\odot} \mathrm{yr}^{-1}\right)$ and terminal velocity $\left(u \simeq 70 \mathrm{~km} \mathrm{~s}^{-1}\right)$ observed for these objects, assuming a weakly damped $\left(L_{0}=5.0 r_{0}\right)$ Alfvén wave flux of $\phi_{\mathrm{A} 0}=10^{7} \mathrm{erg} \mathrm{cm}^{-2} \mathrm{~s}^{-1}$. The velocity profile reveals an efficient acceleration at $r<1.5 r_{0}$, reaching the maximum value $\sim 100 \mathrm{~km} \mathrm{~s}^{-1}$. In this region, the wind is mainly accelerated by the wave energy density and the thermal pressure gradients. Afterwards, the absence of the wave acceleration and the cooling gas result in a decrease of the velocity to the observed values. For the temperature, assuming a weakly damped wave flux, the radiative losses and the expansion cooling are dominant near surface, and the temperature gradient is initially negative. The temperature falls to $\simeq 2500 \mathrm{~K}$ in a sharp region and then, as density decreases as the wind accelerates and the flux tube expands, it increases up to $\simeq 6000 \mathrm{~K}$ at $r<2.0 r_{0}$. For higher distances, where the radiative losses are low and the wave heating is no longer effective, the temperature decreases mainly due to the adiabatic expansion.

\section{ACKNOWLEDGMENTS}

DF-G and AAV thank the Brazilian agency FAPESP for the financial support (04/12053-2 and 04/13846-6). VJ-P thanks CNPq for the financial support (304523/90-9).

\section{REFERENCES}

Airapetian V. S., Carpenter K., Ofman L., 2003, A\&AS, 202, 3214 Axford W. I., McKenzie J. F., 1996, Ap\&SS, 243, 1

Blackman E. G., Frank A., Markiel J. A., Thomas J. H., Van Horn H. M., 2001, Nat, 409, 485

Cranmer S. R., van Ballegooijen A. A., 2005, ApJS, 156, 265

Davila J. M., 1985, ApJ, 291, 328

Dupree A. K., 1986, ARA\&A, 24, 377

Elitzur M., Ivezic Z., 2001, MNRAS, 327, 403

Esser R., Lie-Svendsen O., Janse A. M., Killie M. A., 2005, ApJ, 629, L61

Falceta-Gonç calves D., Jatenco-Pereira V., 2002, ApJ, 576, 976

Guandalini R., Busso M., Ciprini S., Silvestro G., Persi P., 2006, A\&A, 445, 1069

Hartmann L., MacGregor K. B., 1980, ApJ, 242, 260

Hartmann L., Edwards S., Avrett E., 1982, ApJ, 261, 279

Holzer T. E., Fla T., Leer E., 1983, ApJ, 275, 808

Holzer T. E., Leer E., 1980, JGR, 85, 4665

Jatenco-Pereira V., Opher R., 1989, A\&A, 209, 327

Lamers H. J. G. L. M., Cassinelli J. P., 1999, Introduction to Stellar Winds.

Cambridge Univ. Press, New York

Lagage P. O., Cesarsky C. J., 1983, A\&A, 125, 249

Liberatore S., Lafon J., Berruyer N., 2001, A\&A, 377, 522

Parker E. N., 1958, ApJ, 128, 664

Pneuman G. W., Solanki S. K., Stenflo J. O., 1986, A\&A, 154, 231

Sandin C., Hofner S., 2003, A\&A, 404, 789

Schmutzler T., Tscharnuter W. M., 1993, A\&A, 273, 318

Suzuki T. K., Inutsuka S., 2005, ApJ, 632, L49

Tu C., Zhou C., Marsch E., Xia L.-D., Zhao L., Wang J.-X., Wilhelm K., 2005, Sci, 308, 519

Usmanov A. V., Goldstein M. L., Besser B. P., Fritzer J. M., 2000, JGR, 105 , 12675

Vidotto A. A., Jatenco-Pereira V., 2006, ApJ, 639, 416

Vlemmings W. H. T., Diamond P. J., van Lengevelde H. J., 2002, A\&A, 394, 589

Woitke P., Niccolini G., 2005, A\&A, 433, 1101

This paper has been typeset from a $\mathrm{T}_{\mathrm{E}} \mathrm{X} / \mathrm{L} \mathrm{T} \mathrm{T}_{\mathrm{E}} \mathrm{X}$ file prepared by the author. 


\section{Bibliography}

Airapetian, V. S., Ofman, L., Robinson, R. D., Carpenter, K., and Davila, J.: 2000, ApJ 528, 965

Alves, J., Lombardi, M., and Lada, C. J.: 2007, A\&A 462, L17

Andre, P., Montmerle, T., Feigelson, E. D., Stine, P. C., and Klein, K.-L.: 1988, ApJ 335,940

Antoniucci, S., Nisini, B., Giannini, T., and Lorenzetti, D.: 2008, A\&্A 479, 503

Ayres, T. R.: 1997, J. Geophys. Res. 102, 1641

Babcock, H. W.: 1961, ApJ 133, 572

Bagnulo, S., Szeifert, T., Wade, G. A., Landstreet, J. D., and Mathys, G.: 2002, A\&A 389, 191

Baliunas, S. L. and Vaughan, A. H.: 1985, ARA\&A 23, 379

Belcher, J. W. and MacGregor, K. B.: 1976, ApJ 210, 498

Bittencourt, J. A.: 2004, Fundamentals of Plasma Physics, (3rd ed.; Springer-Verlag, New York, USA)

Bouvier, J., Cabrit, S., Fernandez, M., Martin, E. L., and Matthews, J. M.: 1993, Aש̈A 272, 176

Brown, J. M., Blake, G. A., Dullemond, C. P., Merín, B., Augereau, J. C., Boogert, A. C. A., Evans, II, N. J., Geers, V. C., Lahuis, F., Kessler-Silacci, J. E., Pontoppidan, K. M., and van Dishoeck, E. F.: 2007, ApJ 664, L107 
Burrows, C. J., Stapelfeldt, K. R., Watson, A. M., Krist, J. E., Ballester, G. E., Clarke, J. T., Crisp, D., Gallagher, III, J. S., Griffiths, R. E., Hester, J. J., Hoessel, J. G., Holtzman, J. A., Mould, J. R., Scowen, P. A., Trauger, J. T., and Westphal, J. A.: 1996, ApJ 473, 437

Chiosi, C. and Maeder, A.: 1986, ARA $\mathscr{G} A$ 24, 329

Choi, P. I. and Herbst, W.: 1996, AJ 111, 283

Chu, Y.-H., Treffers, R. R., and Kwitter, K. B.: 1983, ApJS 53, 937

Cieza, L. and Baliber, N.: 2007, ApJ 671, 605

Clauer, C., Gombosi, T., De Zeenw, D., Ridley, A., Powell, K., Van Leer, B., Stout, Q., Groth, C., and Holzer, T.: 2000, IEEE Transactions on Plasma Science 28(6), 1931

Code, A. D.: 1973, in B. Hauck and B. E. Westerlund (eds.), Problems of Calibration of Absolute Magnitudes and Temperature of Stars, Vol. 54 of IAU Symposium, pp 131-145

Cohen, O., Sokolov, I. V., Roussev, I. I., Arge, C. N., Manchester, W. B., Gombosi, T. I., Frazin, R. A., Park, H., Butala, M. D., Kamalabadi, F., and Velli, M.: 2007, ApJ 654, L163

Cranmer, S. R.: 2008, ApJ 689, 316

Cranmer, S. R. and van Ballegooijen, A. A.: 2005, ApJS 156, 265

Cranmer, S. R., van Ballegooijen, A. A., and Edgar, R. J.: 2007, ApJS 171, 520

De Zeeuw, D., Gombosi, T., Groth, C., Powell, K., and Stout, Q.: 2000, IEEE Transactions on Plasma Science 28(6), 1956

Donati, J.-F., Forveille, T., Cameron, A. C., Barnes, J. R., Delfosse, X., Jardine, M. M., and Valenti, J. A.: 2006, Science 311, 633

Donati, J.-F., Jardine, M. M., Gregory, S. G., Petit, P., Bouvier, J., Dougados, C., Ménard, F., Cameron, A. C., Harries, T. J., Jeffers, S. V., and Paletou, F.: 2007, MNRAS 380, 1297 
Donati, J.-F., Jardine, M. M., Gregory, S. G., Petit, P., Paletou, F., Bouvier, J., Dougados, C., Ménard, F., Cameron, A. C., Harries, T. J., Hussain, G. A. J., Unruh, Y., Morin, J., Marsden, S. C., Manset, N., Aurière, M., Catala, C., and Alecian, E.: 2008a, MNRAS 386, 1234

Donati, J.-F., Morin, J., Petit, P., Delfosse, X., Forveille, T., Aurière, M., Cabanac, R., Dintrans, B., Fares, R., Gastine, T., Jardine, M. M., Lignières, F., Paletou, F., Velez, J. C. R., and Théado, S.: 2008b, MNRAS 390, 545

Dupree, A. K.: 1986, ARA\&A 24, 377

Dutrey, A., Guilloteau, S., Prato, L., Simon, M., Duvert, G., Schuster, K., and Menard, F.: 1998, A\&GA 338, L63

Edwards, S.: 2009, in E. Stempels (ed.), Proceedings of the 15th Cambridge Workshop on Cool Stars, Stellar Systems and the Sun, Vol. 1094 of American Institute of Physics Conference Series, pp 29-38

Edwards, S., Fischer, W., Kwan, J., Hillenbrand, L., and Dupree, A. K.: 2003, ApJ 599, L41

Falceta-Gonçalves, D., Vidotto, A. A., and Jatenco-Pereira, V.: 2006, MNRAS 368, 1145

Favata, F., Flaccomio, E., Reale, F., Micela, G., Sciortino, S., Shang, H., Stassun, K. G., and Feigelson, E. D.: 2005, ApJS 160, 469

Feldman, U., Doschek, G. A., Schühle, U., and Wilhelm, K.: 1999, ApJ 518, 500

Fisk, L. A. and Zurbuchen, T. H.: 2006, Journal of Geophysical Research (Space Physics) 111, 9115

Fleck, R. C.: 2008, Ap\&SSS 313, 351

Getman, K. V., Feigelson, E. D., Micela, G., Jardine, M. M., Gregory, S. G., and Garmire, G. P.: 2008, ApJ 688, 437

Getman, K. V., Flaccomio, E., Broos, P. S., Grosso, N., Tsujimoto, M., Townsley, L., Garmire, G. P., Kastner, J., Li, J., Harnden, Jr., F. R., Wolk, S., Murray, S. S., Lada, C. J., Muench, A. A., McCaughrean, M. J., Meeus, G., Damiani, F., Micela, G., 
Sciortino, S., Bally, J., Hillenbrand, L. A., Herbst, W., Preibisch, T., and Feigelson, E. D.: 2005, ApJS 160, 319

Ghosh, P. and Lamb, F. K.: 1979, ApJ 234, 296

Goedbloed, J. P. H. and Poedts, S.: 2004, Principles of Magnetohydrodynamics, (Cambridge University Press, Cambridge, UK)

Goldsmith, P. F., Heyer, M., Narayanan, G., Snell, R., Li, D., and Brunt, C.: 2008, ApJ 680, 428

Gombosi, T., Powell, K., De Zeeuw, D., Clauer, C., Hansen, K., Manchester, W., Ridley, A., Roussev, I., Sokolov, I., Stout, Q., and Toth, G.: 2004, Computing in Science 86 Engineering 6(2), 14

Gombosi, T. I.: 1998, Physics of the Space Environment, Cambridge atmospheric and space science series, (Cambridge University Press, New York, USA)

Gombosi, T. I., Powell, K. G., and de Zeeuw, D. L.: 1994, J. Geophys. Res. 99, 21525

Gómez de Castro, A. I. and Verdugo, E.: 2007, ApJ 654, L91

Goodwin, S. P., Whitworth, A. P., and Ward-Thompson, D.: 2004, A\&A 414, 633

Grail, R. R., Coles, W. A., Klinglesmith, M. T., Breen, A. R., Williams, P. J. S., Markkanen, J., and Esser, R.: 1996, Nature 379, 429

Gregory, S. G., Matt, S. P., Donati, J.-F., and Jardine, M.: 2008, MNRAS 389, 1839

Groth, C. P. T., De Zeeuw, D. L., Gombosi, T. I., and Powell, K. G.: 2000, J. Geophys. Res. 105, 25053

Güdel, M., Briggs, K. R., Arzner, K., Audard, M., Bouvier, J., Feigelson, E. D., Franciosini, E., Glauser, A., Grosso, N., Micela, G., Monin, J.-L., Montmerle, T., Padgett, D. L., Palla, F., Pillitteri, I., Rebull, L., Scelsi, L., Silva, B., Skinner, S. L., Stelzer, B., and Telleschi, A.: 2007, A\&A 468, 353

Hansen, K. C., Ridley, A. J., Hospodarsky, G. B., Achilleos, N., Dougherty, M. K., Gombosi, T. I., and Tóth, G.: 2005, Geophys. Res. Lett. 32, 20

Hartigan, P., Edwards, S., and Ghandour, L.: 1995, ApJ 452, 736 
Hartmann, L., Hewett, R., and Calvet, N.: 1994, ApJ 426, 669

Hartmann, L. and Kenyon, S. J.: 1996, ARA\&A 34, 207

Hartmann, L. and Stauffer, J. R.: 1989, AJ 97, 873

Hayashi, C. and Nakano, T.: 1963, Progress of Theoretical Physics 30, 460

Hébrard, G., Bouchy, F., Pont, F., Loeillet, B., Rabus, M., Bonfils, X., Moutou, C., Boisse, I., Delfosse, X., Desort, M., Eggenberger, A., Ehrenreich, D., Forveille, T., Lagrange, A.-M., Lovis, C., Mayor, M., Pepe, F., Perrier, C., Queloz, D., Santos, N. C., Ségransan, D., Udry, S., and Vidal-Madjar, A.: 2008, A\&A 488, 763

Herbig, G. H.: 1962, Advances in Astronomy and Astrophysics 1, 47

Herbst, W., Bailer-Jones, C. A. L., Mundt, R., Meisenheimer, K., and Wackermann, R.: 2002, A\&A 396, 513

Hollenbach, D. J., Yorke, H. W., and Johnstone, D.: 2000, in Mannings, V., Boss, A. P., and Russell, S. S. (eds.), Protostars and Planets IV, pp 401-428

Holzwarth, V.: 2005, A\&A 440, 411

Hubrig, S., Pogodin, M. A., Yudin, R. V., Schöller, M., and Schnerr, R. S.: 2007, AשA 463, 1039

Hussain, G. A. J., Collier Cameron, A., Jardine, M. M., Dunstone, N., Velez, J. R., Stempels, H. C., Donati, J.-F., Semel, M., Aulanier, G., Harries, T., Bouvier, J., Dougados, C., Ferreira, J., Carter, B. D., and Lawson, W. A.: 2009, MNRAS 398, 189 Iben, I. J.: 1967, ARA\&A 5, 571

Jardine, M. and van Ballegooijen, A. A.: 2005, MNRAS 361, 1173

Jardine, M. M., Gregory, S. G., and Donati, J.-F.: 2008, MNRAS 386, 688

Jatenco-Pereira, V. and Opher, R.: 1989, A\&A 209, 327

Jeans, J. H.: 1902, Royal Society of London Philosophical Transactions Series A 199, 1 Johns-Krull, C. M.: 2007, ApJ 664, 975 
Johns-Krull, C. M. and Herczeg, G. J.: 2007, ApJ 655, 345

Johns-Krull, C. M., Valenti, J. A., and Koresko, C.: 1999, ApJ 516, 900

Jones, G. H., Balogh, A., and Forsyth, R. J.: 1998, Geophys. Res. Lett. 25, 3109

Kawaler, S. D.: 1988, ApJ 333, 236

Kemper, F., Stark, R., Justtanont, K., de Koter, A., Tielens, A. G. G. M., Waters, L. B. F. M., Cami, J., and Dijkstra, C.: 2003, A\&\&A 407, 609

Keppens, R. and Goedbloed, J. P.: 1999, A\&A 343, 251

Keppens, R. and Goedbloed, J. P.: 2000, ApJ 530, 1036

Koenigl, A.: 1991, ApJ 370, L39

Koenigl, A. and Pudritz, R. E.: 2000, in Mannings, V., Boss, A. P., and Russell, S. S. (eds.), Protostars and Planets IV, pp 759-788

Kraft, R. P.: 1967, ApJ 150, 551

Kuznetsova, M. M., Hesse, M., Rastätter, L., Taktakishvili, A., Toth, G., De Zeeuw, D. L., Ridley, A., and Gombosi, T. I.: 2007, Journal of Geophysical Research (Space Physics) 112, 10210

Kwan, J., Edwards, S., and Fischer, W.: 2007, ApJ 657, 897

Lagadec, E., Zijlstra, A. A., Matsuura, M., Menzies, J. W., van Loon, J. T., and Whitelock, P. A.: 2008, MNRAS 383, 399

Lamm, M. H., Mundt, R., Bailer-Jones, C. A. L., and Herbst, W.: 2005, A\&̊A 430, 1005

Landin, N. R., Mendes, L. T. S., and Vaz, L. P. R.: 2005, in E. M. de Gouveia dal Pino, G. Lugones, and A. Lazarian (eds.), Magnetic Fields in the Universe: From Laboratory and Stars to Primordial Structures., Vol. 784 of American Institute of Physics Conference Series, pp 607-612

Larson, R. B.: 1981, MNRAS 194, 809

Lazarian, A. and Opher, M.: 2009, ApJ 703, 8 
Levrard, B., Winisdoerffer, C., and Chabrier, G.: 2009, ApJ 692, L9

Lima, J. J. G., Priest, E. R., and Tsinganos, K.: 2001, A\&\&A 371, 240

Lin, D. N. C., Bodenheimer, P., and Richardson, D. C.: 1996, Nature 380, 606

Lin, J., Ko, Y.-K., Sui, L., Raymond, J. C., Stenborg, G. A., Jiang, Y., Zhao, S., and Mancuso, S.: 2005, ApJ 622, 1251

Linde, T. J.: 1998, Ph.D. thesis, University Of Michigan

Linde, T. J., Gombosi, T. I., Roe, P. L., Powell, K. G., and Dezeeuw, D. L.: 1998, J. Geophys. Res. 103, 1889

Liu, Y. C.-M., Opher, M., Cohen, O., Liewer, P. C., and Gombosi, T. I.: 2008, ApJ 680, 757

Lovelace, R. V. E., Romanova, M. M., and Barnard, A. W.: 2008, MNRAS 389, 1233

Low, B. C. and Tsinganos, K.: 1986, ApJ 302, 163

Lugaz, N., Manchester, IV, W. B., and Gombosi, T. I.: 2005, ApJ 627, 1019

Maeder, A. and Meynet, G.: 2000, A\&̈A 361, 159

Manchester, W. B., Gombosi, T. I., Roussev, I., De Zeeuw, D. L., Sokolov, I. V., Powell, K. G., Tóth, G., and Opher, M.: 2004, Journal of Geophysical Research (Space Physics) 109, 1102

Massi, M., Ros, E., Menten, K. M., Kaufman Bernadó, M., Torricelli-Ciamponi, G., Neidhöfer, J., Boden, A., Boboltz, D., Sargent, A., and Torres, G.: 2008, A\&\& 480, 489

Matt, S. and Pudritz, R. E.: 2005, ApJ 632, L135

Matt, S. and Pudritz, R. E.: 2008a, ApJ 678, 1109

Matt, S. and Pudritz, R. E.: 2008b, ApJ 681, 391

Matt, S. and Pudritz, R. E.: 2008c, in G. van Belle (ed.), Proceedings of the 14 th Cambridge Workshop on Cool Stars, Stellar Systems and the Sun, Vol. 384 of Astronomical Society of the Pacific Conference Series, pp 339-348 
Matthaeus, W. H., Elliott, H. A., and McComas, D. J.: 2006, Journal of Geophysical Research (Space Physics) 111, 10103

Matthaeus, W. H., Zank, G. P., Oughton, S., Mullan, D. J., and Dmitruk, P.: 1999, ApJ 523, L93

McComas, D. J., Bame, S. J., Barraclough, B. L., Feldman, W. C., Funsten, H. O., Gosling, J. T., Riley, P., Skoug, R., Balogh, A., Forsyth, R., Goldstein, B. E., and Neugebauer, M.: 1998, Geophys. Res. Lett. 25, 1

McComas, D. J., Barraclough, B. L., Gosling, J. T., Hammond, C. M., Phillips, J. L., Neugebauer, M., Balogh, A., and Forsyth, R. J.: 1995, J. Geophys. Res. 100, 19893

Mestel, L.: 1968, MNRAS 138, 359

Moutou, C., Hébrard, G., Bouchy, F., Eggenberger, A., Boisse, I., Bonfils, X., Gravallon, D., Ehrenreich, D., Forveille, T., Delfosse, X., Desort, M., Lagrange, A.-M., Lovis, C., Mayor, M., Pepe, F., Perrier, C., Pont, F., Queloz, D., Santos, N. C., Ségransan, D., Udry, S., and Vidal-Madjar, A.: 2009, A\&A 498, L5

Mullan, D. J., Doyle, J. G., Redman, R. O., and Mathioudakis, M.: 1992, ApJ 397, 225

Mullan, D. J., Sion, E. M., Bruhweiler, F. C., and Carpenter, K. G.: 1989, ApJ 339, L33

Neugebauer, M. and Snyder, C. W.: 1962, Science 138, 1095

Opher, M., Liewer, P. C., Gombosi, T. I., Manchester, W., DeZeeuw, D. L., Sokolov, I., and Toth, G.: 2003, ApJ 591, L61

Opher, M., Liewer, P. C., Velli, M., Bettarini, L., Gombosi, T. I., Manchester, W., DeZeeuw, D. L., Toth, G., and Sokolov, I.: 2004, ApJ 611, 575

Opher, M., Stone, E. C., and Liewer, P. C.: 2006, ApJ 640, L71

Orlando, S., Peres, G., and Reale, F.: 2000, ApJ 528, 524

Osterbrock, D. E.: 1961, ApJ 134, 347

Papaloizou, J. C. B.: 2007, A\&A 463, 775

Papaloizou, J. C. B. and Nelson, R. P.: 2005, A\&A 433, 247 
Parker, E. N.: 1955, ApJ 122, 293

Parker, E. N.: 1958, ApJ 128, 664

Pasachoff, J. M.: 2009, Nature 459, 789

Pascucci, I., Gorti, U., Hollenbach, D., Najita, J., Meyer, M. R., Carpenter, J. M., Hillenbrand, L. A., Herczeg, G. J., Padgett, D. L., Mamajek, E. E., Silverstone, M. D., Schlingman, W. M., Kim, J. S., Stobie, E. B., Bouwman, J., Wolf, S., Rodmann, J., Hines, D. C., Lunine, J., and Malhotra, R.: 2006, ApJ 651, 1177

Peres, G., Orlando, S., Reale, F., Rosner, R., and Hudson, H.: 2000, ApJ 528, 537

Petit, P., Dintrans, B., Solanki, S. K., Donati, J.-F., Aurière, M., Lignières, F., Morin, J., Paletou, F., Ramirez Velez, J., Catala, C., and Fares, R.: 2008, MNRAS 388, 80

Petit, P., Donati, J.-F., Aurière, M., Landstreet, J. D., Lignières, F., Marsden, S., Mouillet, D., Paletou, F., Toqué, N., and Wade, G. A.: 2005, MNRAS 361, 837

Pevtsov, A. A., Fisher, G. H., Acton, L. W., Longcope, D. W., Johns-Krull, C. M., Kankelborg, C. C., and Metcalf, T. R.: 2003, ApJ 598, 1387

Phan-Bao, N., Martín, E. L., Donati, J.-F., and Lim, J.: 2006, ApJ 646, L73

Phillips, J. L., Bame, S. J., Barnes, A., Barraclough, B. L., Feldman, W. C., Goldstein, B. E., Gosling, J. T., Hoogeveen, G. W., McComas, D. J., Neugebauer, M., and Suess, S. T.: 1995, Geophys. Res. Lett. 22, 3301

Phillips, J. P. and Ramos-Larios, G.: 2008, MNRAS 383, 1029

Phillips, R. B., Lonsdale, C. J., and Feigelson, E. D.: 1991, ApJ 382, 261

Phillips, R. B., Lonsdale, C. J., Feigelson, E. D., and Deeney, B. D.: 1996, AJ 111, 918 Pneuman, G. W. and Kopp, R. A.: 1971, Sol. Phys. 18, 258

Pollack, J. B., Hubickyj, O., Bodenheimer, P., Lissauer, J. J., Podolak, M., and Greenzweig, Y.: 1996, Icarus 124, 62

Portinari, L., Chiosi, C., and Bressan, A.: 1998, A\&A 334, 505 
Powell, K. G.: 1994, An Approximate Riemann Solver For Magnetohydrodynamics (That Works in More than One Dimension), Technical report, Institute for Computer Applications in Science and Engineering (ICASE), No. 94-24

Powell, K. G., Roe, P. L., Linde, T. J., Gombosi, T. I., and de Zeeuw, D. L.: 1999, Journal of Computational Physics 154, 284

Preibisch, T., Kim, Y.-C., Favata, F., Feigelson, E. D., Flaccomio, E., Getman, K., Micela, G., Sciortino, S., Stassun, K., Stelzer, B., and Zinnecker, H.: 2005, ApJS 160, 401

Rebull, L. M., Stauffer, J. R., Megeath, S. T., Hora, J. L., and Hartmann, L.: 2006, ApJ 646, 297

Reiners, A. and Basri, G.: 2006, ApJ 644, 497

Reipurth, B., Yu, K. C., Rodríguez, L. F., Heathcote, S., and Bally, J.: 1999, A $\& A 352$, L83

Ridley, A. J., de Zeeuw, D. L., Manchester, W. B., and Hansen, K. C.: 2006, Advances in Space Research 38, 263

Robinson, R. D., Worden, S. P., and Harvey, J. W.: 1980, ApJ 236, L155

Romanova, M. M. and Lovelace, R. V. E.: 2006, ApJ 645, L73

Roussev, I. I., Gombosi, T. I., Sokolov, I. V., Velli, M., Manchester, IV, W., DeZeeuw, D. L., Liewer, P., Tóth, G., and Luhmann, J.: 2003, ApJ 595, L57

Safier, P. N.: 1998, ApJ 494, 336

Sahai, R., Morris, M., Sánchez Contreras, C., and Claussen, M.: 2007, AJ 134, 2200

Sauty, C. and Tsinganos, K.: 1994, A\&A 287, 893

Scelsi, L., Maggio, A., Micela, G., Briggs, K., and Güdel, M.: 2007, A\&AA 473, 589

Schaller, G., Schaerer, D., Meynet, G., and Maeder, A.: 1992, A\&AS 96, 269

Schmitt, J. H. M. M.: 1997, A\&A 318, 215

Schmitt, J. H. M. M. and Wichmann, R.: 2001, Nature 412, 508 
Schmutzler, T. and Tscharnuter, W. M.: 1993, A\&A 273, 318

Schrijver, C. J.: 2001, ApJ 547, 475

Shu, F., Najita, J., Ostriker, E., Wilkin, F., Ruden, S., and Lizano, S.: 1994, ApJ 429, 781

Silvester, J., Neiner, C., Henrichs, H. F., Wade, G. A., Petit, V., Alecian, E., Huat, A.-L., Martayan, C., Power, J., and Thizy, O.: 2009, MNRAS 398, 1505

Skelly, M. B., Unruh, Y. C., Cameron, A. C., Barnes, J. R., Donati, J.-F., Lawson, W. A., and Carter, B. D.: 2008, MNRAS 385, 708

Skumanich, A.: 1972, ApJ 171, 565

Steinolfson, R. S. and Hundhausen, A. J.: 1988, J. Geophys. Res. 93, 14269

Suess, S. T. and Smith, E. J.: 1996, Geophys. Res. Lett. 23, 3267

Susino, R., Ventura, R., Spadaro, D., Vourlidas, A., and Landi, E.: 2008, A\& $A$ 488, 303

Suzuki, T. K.: 2007, ApJ 659, 1592

Suzuki, T. K. and Inutsuka, S.-i.: 2005, ApJ 632, L49

Telleschi, A., Güdel, M., Briggs, K. R., Audard, M., Ness, J.-U., and Skinner, S. L.: 2005, ApJ 622, 653

Telleschi, A., Güdel, M., Briggs, K. R., Audard, M., and Palla, F.: 2007, A $\mathscr{G} A$ 468, 425

Tóth, G.: 2000, Journal of Computational Physics 161, 605

Tóth, G., de Zeeuw, D. L., Gombosi, T. I., and Powell, K. G.: 2006, Journal of Computational Physics 217, 722

Tóth, G., Kovács, D., Hansen, K. C., and Gombosi, T. I.: 2004, Journal of Geophysical Research (Space Physics) 109, 11210

Tóth, G., Sokolov, I. V., Gombosi, T. I., Chesney, D. R., Clauer, C. R., De Zeeuw, D. L., Hansen, K. C., Kane, K. J., Manchester, W. B., Oehmke, R. C., Powell, K. G., Ridley, A. J., Roussev, I. I., Stout, Q. F., Volberg, O., Wolf, R. A., Sazykin, S., Chan, A., Yu, B., and Kóta, J.: 2005, Journal of Geophysical Research (Space Physics) 110, 12226 
Totten, T. L., Freeman, J. W., and Arya, S.: 1995, J. Geophys. Res. 100, 13

Trilling, D. E., Benz, W., Guillot, T., Lunine, J. I., Hubbard, W. B., and Burrows, A.: 1998, ApJ 500, 428

Tsinganos, K. and Trussoni, E.: 1991, A\&A 249, 156

Tu, C.-Y., Zhou, C., Marsch, E., Xia, L.-D., Zhao, L., Wang, J.-X., and Wilhelm, K.: 2005, Science 308, 519

ud-Doula, A.: 2003, Ph.D. thesis, University Of Delaware

ud-Doula, A. and Owocki, S. P.: 2002, ApJ 576, 413

Usmanov, A. V. and Goldstein, M. L.: 2003, Journal of Geophysical Research (Space Physics) 108, 1354

Valenti, J. A. and Johns-Krull, C. M.: 2004, Ap\&SS 292, 619

Valyavin, G., Wade, G. A., Bagnulo, S., Szeifert, T., Landstreet, J. D., Han, I., and Burenkov, A.: 2008, ApJ 683, 466

Velli, M.: 1994, ApJ 432, L55

Vidotto, A. A., Falceta-Gonçalves, D., and Jatenco-Pereira, V.: 2006, Space Science Reviews 122, 181

Vidotto, A. A. and Jatenco-Pereira, V.: 2006, ApJ 639, 416

Vidotto, A. A. and Jatenco-Pereira, V.: 2009, Advances in Space Research, In Press

Vidotto, A. A., Opher, M., Jatenco-Pereira, V., and Gombosi, T. I.: 2009a, ApJ 699, 441

Vidotto, A. A., Opher, M., Jatenco-Pereira, V., and Gombosi, T. I.: 2009b, ApJ 703, 1734

Wade, G. A., Bagnulo, S., Drouin, D., Landstreet, J. D., and Monin, D.: 2007, MNRAS 376,1145

Walter, F. M., Brown, A., Mathieu, R. D., Myers, P. C., and Vrba, F. J.: 1988, AJ 96, 297 
Washimi, H. and Shibata, S.: 1993, MNRAS 262, 936

Weber, E. J. and Davis, L. J.: 1967, ApJ 148, 217

Wilhelm, K.: 2006, A\&A 455, 697

Winn, J. N., Johnson, J. A., Fabrycky, D., Howard, A. W., Marcy, G. W., Narita, N., Crossfield, I. J., Suto, Y., Turner, E. L., Esquerdo, G., and Holman, M. J.: 2009, ApJ 700, 302

Wolk, S. J. and Walter, F. M.: 1996, AJ 111, 2066

Wood, B. E.: 2004, Living Reviews in Solar Physics 1, 2

Wood, B. E., Müller, H.-R., Zank, G. P., and Linsky, J. L.: 2002, ApJ 574, 412

Wood, B. E., Müller, H.-R., Zank, G. P., Linsky, J. L., and Redfield, S.: 2005, ApJ 628, L143

Wood, D. O. S. and Churchwell, E.: 1989, ApJS 69, 831

Yang, H., Johns-Krull, C. M., and Valenti, J. A.: 2008, AJ 136, 2286

Zinnecker, H., McCaughrean, M. J., and Rayner, J. T.: 1998, Nature 394, 862

Zolensky, M. E., Bodnar, R. J., Gibson, Jr., E. K., Nyquist, L. E., Reese, Y., Shih, C.-Y., and Wiesmann, H.: 1999, Science 285, 1377

Zurbuchen, T. H.: 2007, ARA\&A 45, 297 
